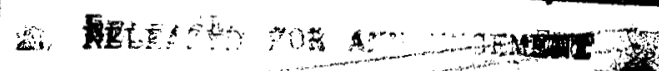

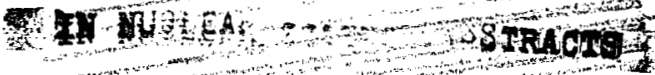

SAN ONOFRE

NUCLEAR GENERATING STATION

UNIT !

FINAL ENGINEERING REPORT.

AND

\title{
SAFETY ANALYSIS
}

\section{VOLUME III}

LEGAL NOTICE

This report was prepared as an account of Government sponsored work. Neither the United States, not the Commission, not any person acting on behalf of the Commission:

A. Makes any warranty or representation, expressed or implied, with respect to the accuracy, completeness, or usefulness of the information contained in this report, or that the use of any information, apparatus, method, of process disclosed in this report may not infringe privately owned rights; or $B$. Assumes any liabilities wtth respect to the use of, or for dameges resulting from the use of any Assumes any liabilities with respect to the use of, or for dame
information, apparatus, method, or process disclosed in this report.

As used in the above, "person acting on behalf of the Commls sion" includes any employee or conteactor of the Commission, of employee of such contractor, to the extent that such employee or contractor of the Commission, or employee of such contractor prepares, disseminates, or provides access to, any information pursuant to his employment or contract with the Commission, or his employment with such contractor.

Submitted in compliance with the requirements of Contract AT(30-1)-3270.

\section{SOUTHERN CALIFORNIA EDISON COMPANY}

\section{SAN DIEGO GAS \& ELECTRIC COMPANY}




\section{DISCLAIMER}

This report was prepared as an account of work sponsored by an agency of the United States Government. Neither the United States Government nor any agency Thereof, nor any of their employees, makes any warranty, express or implied, or assumes any legal liability or responsibility for the accuracy, completeness, or usefulness of any information, apparatus, product, or process disclosed, or represents that its use would not infringe privately owned rights. Reference herein to any specific commercial product, process, or service by trade name, trademark, manufacturer, or otherwise does not necessarily constitute or imply its endorsement, recommendation, or favoring by the United States Government or any agency thereof. The views and opinions of authors expressed herein do not necessarily state or reflect those of the United States Government or any agency thereof. 


\section{DISCLAIMER}

Portions of this document may be illegible in electronic image products. Images are produced from the best available original document. 
INTRODUCTION

Scope and Definition

SECTION 1 - SUMMARY

1.1 General Description 1-1

1.1.1 Site 1-1

1.1.2 Design Capability $1-1$

1.1.3 Reactor Core 1-1

1.1.4 Coolant System 1-2

1.1.5 Chemical and Volume Control System 1-3

1.1.6 Auxiliary Coolant System 1-3

1.1.7 Sampling and Health Physics 1-3

1.1.8 Fuel Handling and Storage $1-3$

1.1.9 Radioactive Waste Treatment 1-4

1.1.10 Turbine Cycle $1-5$

1.1.11 Electrical System 1-5

1.1.12 Miscellaneous Systems 1-6

1.1.13 Safety Injection System 1-7

$\begin{array}{ll}1.1 .14 & \text { Shielding } \\ 1-7\end{array}$

1.1.15 Containment Sphere 1-8

1.1.16 Control and Instrumentation 1-8

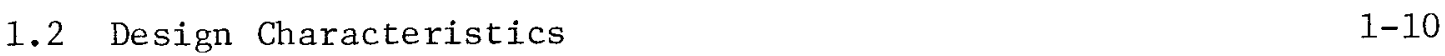

$\begin{array}{ll}1.2 .1 \text { General } & 1-10\end{array}$

1.2.2 Core Mechanical Design Parameters 1-10

1.2.3 Core Thermal - Hydraulic Design Parameters 1-11

1.2.4 Nuclear Design Data 1-12

1.3 Safety Principles 1-14

1.3.1 General 1-14

1.3.2 Site Selection 1-14

1.3.3 Inherent Safety Characteristics $1-14$

1.3.4 Engineering Safety Features $1-14$

1.3.5 Safety Evaluation and Containment 1-16

$\begin{array}{lll}1.4 & \text { General Conclusions } & 1-17\end{array}$

SECTION 2 - SITE DESCRIPTION

2.1 Site and Adjacent Areas 2-1

2.1.1 General Description 2-1 
Page

2.1.2 Site Plot Plan 2-5

2.1.3 Grading 2-5

2.1.4 Ground Water 2-9

2.2 Meteorology 2-11

2.2.1 General Meteorological Conditions 2-11

2.2.2 Wind Movement 2-13

2.2.3 Atmospheric Stability 2-33

2.2.4 Dispersion of Pollutants Released at Site 2-37

2.2.5 Conclusions $2-40$

2.2.6 Meteorological Data From the Site and San Clemente 2-40

2.2.7 References and Selected Bibliography 2-56

$\begin{array}{llr}2.3 \text { Geology } & 2-57\end{array}$

2.3.1 General 2-57

2.3.2 Subsurface Investigation by Borings 2-57

2.3.3 Refraction Surveys 2-60

2.3.4 Laboratory Tests 2-61

2.3.5 Foundations 2-61

2.3.6 Faults $2-62$

2.3.7 Conclusions $2-65$

2.3.8 Bibliography 2-65

$\begin{array}{ll}2.4 \text { Hydrology } & 2-67\end{array}$

2.5 Seismology 2-68
2 2-

2.5.1 Description of Major Shocks 2-68

2.5.2 Site Seismicity $2-73$

2.5.3 Ground Motion Studies 2-79

2.5.4 Installation of Strong Motion Recorder 2-85

2.5.5 Definition of Terms 2-85

$\begin{array}{ll}2.5 .6 & \text { References }\end{array}$

$\begin{array}{llr}2.6 & \text { Oceanography } & 2-89\end{array}$

2.6.1 Beneficial Uses 2-89

2.6.2 Currents 2-89

2.6.3 Tides and Waves 2-91

2.6.4 Bottom Conditions 2-92

2.6.5 Water Temperatures 2-92

2.6.6 References $2-92$

$\begin{array}{llr}2.7 & \text { Tsunami } & 2-94\end{array}$

2.7.1 General $2-94$

2.7.2 AEC Staff Questions 2-96 
2.8.1 General

SECTION 3 - PLANT FACILITIES - REACTOR CYCLE

3.1 General Description 3-1

3.1.1 Scope $3-1$

3.1.2 Nuclear Steam Supply System 3-1

3.1.3 Turbine Cycle 3-2

3.1.4 Control-Administration Building 3-2

3.1.5 Plant Arrangement 3-2

3.2 Reactor Mechanical Design 3-8

3.2.1 General Description 3-8

3.2.2 Fuel Pellets 3-12

3.2.3 Fue1 Rods 3-13

3.2.4 Fuel Assembly 3-13

3.2.5 Rod Cluster Control Assembly 3-21

3.2.6 Prototype Testing 3-23

3.2.7 Reactor Internals 3-24

3.2.8 Control Rod Drive Mechanisms 3-29

3.2.9 Drive Mechanism Materials 3-32

3.2.10 Principles of Operation 3-32

3.2.11 Reference 3-34

3.3 Reactor Thermal-Hydraulic Design 3-35

3.3.1 General 3-35

3.3.2 Coolant Flow 3-35

3.3.3 Variation of Primary System Temperature and $3-36$ Pressure $3-36$

3.3.4 Hot Channel Factors 3-36

3.3.5 Bulk Boiling 3-39

3.3.6 Departure From Nucleate Boiling (DNB) 3-39

3.3.7 Film Boiling Heat Transfer Coefficient 3-49

3.3.8 Central Temperature of the Hot Pellet 3-49

3.3.9 Hydraulic and Thermal Design Parameters 3-53

3.3.10 References 3-54

3.4 Reactor Nuclear Design 3-55

3.4.1 General 3-55

3.4.2 Core Description 3-55

3.4.3 Nuclear Design Characteristics 3-58 


\section{CONTENTS (Continued)}

$\underline{\text { Page }}$

3.4.4 Reactivity Control Aspects 3-62

3.4.5 Reactor Core Power Distribution 3-72

3.4.6 Analyses and Procedures 3-77

3.4.7 Reactivity Coefficients 3-90

3.4.8 References 3-102

3.5 Reactor Coolant System 3-103

3.5.1 General Description 3-103

3.5.2 Equipment Description 3-110

3.6 Chemical and Volume Control System 3-123

3.6.1 General Description 3-123

3.6.2 Equipment Description 3-132

3.7 Auxiliary Coolant System 3-142

3.7.1 General Description 3-142

3.7.2 Equipment Description 3-146

3.7.3 Code Requirements 3-150

3.8 Sampling System 3-151

3.8.1 General Description 3-151

3.8.2 Equipment Description 3-153

3.9 Fuel Handling and Transfer System 3-155

3.9.1 General Description 3-155

3.9.2 Equipment Description and Design Basis 3-159

3.10 Radioactive Waste Disposal System 3-167

3.10.1 General 3-167

3.10.2 Liquid Radioactive Coolant Wastes 3-169

3.10.3 Solid Radioactive Wastes 3-182

3.10.4 Gaseous Radioactive Waste 3-183

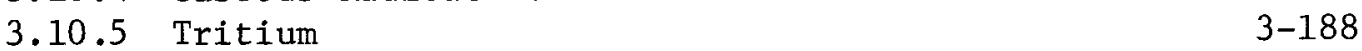

SECTION 4 - PLANT FACILITIES - TURBINE CYCLE

4.1 Turbine Cycle 4-1

4.1.1 General Description 4-1

4.1.2 Turbine-Generator: 4-1

4.1.3 Main Condensers 4-16

4.1.4 Steam System $\quad 4-17$

4.1.5 Feedwater and Condensate System 4-18 


\section{CONTENTS (Continued)}

\subsubsection{Circulating Water System}

Page

4.2 Electrical Systems $\quad$ 4-21

4.2.1 General 4-21

4.2.2 220-KV and 138-KV Systems 4-21

4.2.3 Auxiliary Electric Power Systems. 4-35

4.2.4 4160-V and 480-V Systems 4-35

4.2.5 $120-V$ D $-C$ and $120-V$ A-C Maintained Control
Power Systems

4.2.6 Annunciator System 4-44

4.2.7 Communication Systems $\quad 4-44$

4.2.8 Lighting System 4-44

4.2.9 Cable System $\quad$ 4-45

4.2.10 Initial Performance Tests 4-46

$\begin{array}{lll}4.3 & \text { Control Room } & 4-47\end{array}$

4.3.1 General Description 4-47

4.3.2 Annunciators $\quad 4-54$

4.3.3 Accessory Equipment $\quad 4-54$

4.4 Plant Services 4-56

4.4.1 General 4-56

4.4.2 Miscellaneous Water Services 4-56

4.4.3 Compressed Air System 4-60

SECTION 5 - PLANT FACILITIES - SAFETY SYSTEMS

5.1 Safety Injection System 5-1

5.1.1 Function 5-1

5.1.2 System Design 5-1

5.1.3 General Description 5-2

5.1.4 Equipment Description 5-9

5.1.5 Safeguards Against Accidental Injection of $5-16$

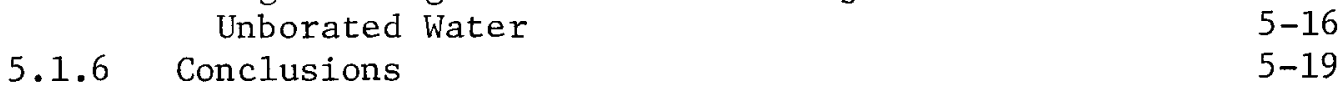

5.2 Shielding 5-21

5.2.1 General 5-21

5.2.2 Design Criteria for Radiation Zones at Full-Power
Operation and After Shutdown

5.2.3 Design Criteria for Radiation Level Inside the
Containment Sphere

5.2.4 Primary Shield 5-29

5.2.5 Secondary Shield 5-31 


\section{CONTENTS (Continued)}

5.2.6 Fuel Handling Shield

$\underline{\text { Page }}$

5.2.7 Reactor Auxiliary Building Shielding 5-35

5.2.8 Auxiliary Shielding

$5-35$

5.3 Containment Sphere $5-36$

5.3.1 Purpose and Description 5-36

5.3.2 Design Criteria 5-36

5.3.3 Method of Analysis 5-37

5.3.4 Results and Conclusions $5-40$

5.3.5 Sphere Instrumentation 5-44

5.3.6 Penetrations 5-44

5.3.7 Access 5-55

5.3.8 Pressure and Leak-Rate Testing Prior to Operation 5-55

5.3.9 Provisions for Future Testing 5-57

SECTION 6 - INSTRUMENTATION AND CONTROL SYSTEMS

6.1 Nuclear Instrumentation System 6-1

6.1.1 Introduction 6-1

6.1.2 Genera1 Description 6-1

6.1.3 Description of Components 6-7

6.1.4 System Characteristics 6-13

6.2 Reactor Control and Protection System 6-15

6.2.1 General Description $\quad 6-15$

6.2.2 Reactor Control System $6-15$

6.2.3 Reactor Protection System 6-27

6.2.4 Reactor Control System Functional Description 6-35

6.2.5 Permissive Circuit Units 6-39

$\begin{array}{ll}\text { 6.3 Rod Drive Control System } & 6-43\end{array}$

6.3.1 General Description 6-43

6.3.2 Description of Equipment 6-50

6.4 Rod Position Indicating System 6-52

6.4.1 General Description 6-52

6.4.2 Description of Equipment 6-52

6.5 Steam Generator Leve1 Control System 6-55

6.5.1 System Description 6-55

6.5.2 Function and Override Characteristics 6-55

6.5.3 Feedwater Control 6-57 
6.6 In-Core Instrumentation System 6-58

6.6.1 General Description 6-58

6.6.2 Aero-Ba11 F1ux Mapping System 6-58

6.6.3 Thermocouples 6-62

6.6.4 Handling During Refueling 6-63

6.6.5 Core Pattern Selection and Application 6-63

6.6.6 Equipment Description 6-64

6.6.7 Seismic Design 6-65

$\begin{array}{llr}6.7 & \text { Radiation Monitoring System } & 6-67\end{array}$

6.7.1 General Description 6-67

6.7.2 Operational Radiation Monitoring System 6-67

6.7.3 Area Radiation Monitoring System 6-71

6.7.4 Equipment Description 6-71

6.7.5 Seismic Conditions 6-79

6.8 Health Physics and Laboratory Equipment 6-80

6.8.1 General Description $\quad 6-80$

6.8.2 Description of Equipment 6-80

SECTION 7 - ADMINISTRATION

7.1 Organization and Training 7-1

7.1.1 General 7-1

7.1.2 Operation $7-3$

7.1.3 Plant Technical Staff 7-3

7.1 .4 Station Maintenance 7-4

7.1.5 Personnel Previous Training and Background 7-4

7.1.6 Nuclear Training Program 7-5

7.1.7 Operating Procedures 7-11

7.1.8 Records 7-11

7.2 Testing and Initial Operation 7-12

7.2.1 General 7-12

7.2.2 Phase I - Pre-Critical Testing and Operation 7-12

7.2.3 Phase II - Initial Core Loading and Zero Power
Tests

7.2.4 Phase III - Power Testing 7-16

SECTION 8 - RESEARCH AND DEVELOPMENT

8.1 Research and Development 8-1
8.1.1 General
$8-1$ 
8.1.2 Development of Computational Techniques

Page

8.1.3 Performance of Critical Experiments

8.1.4 Development of New Fuel Assembly Design.

8.1.5 Development of Chemical Shim Techniques

8.1.6 Development of Plant Control System

$8-1$

$8-2$

$8-2$

$8-4$

$8-5$

SECTION 9 - REACTIVITY FAULT ANALYSES

\subsection{Startup}

$9-1$

9.1.1 Genera1

9.1.2 Facility Design Aspects

9.1.3 Conclusions

$9-1$

$9-13$

9.2 Rod Withdrawa1 At Power

$9-14$

9.2.1 General

$9-14$

9.2.2 Facility Design Aspects 9-14

9.2.3 Conclusions

$9-23$

9.3 Boron Dilution

$9-26$

9.3.1 General

$9-26$

9.3.2 Facility Design Aspects

$9-26$

9.3.3 Conclusions

$9-30$

9.4 Cold Water

$9-31$

9.4.1 General

9.4.2 Startup of an Inactive Loop

$9-31$

$9-31$

9.4.3 Addition of Excess Feedwater

$9-33$

9.4.4 Large Load Increases

$9-36$

9.4.5 Conclusions

$9-41$

9.5 Dropped Rod

9.42

9.5.1 General

9.5.2 Facility Design Aspects

9.5.3 Conclusions

$9-42$

$9-42$

$9-45$

$\begin{array}{llr}9.6 & \text { Control Rod Ejection Accident } & 9-47\end{array}$

$\begin{array}{ll}9.6 .1 & \text { General } \\ 9-47\end{array}$

$\begin{array}{lr}\text { 9.6.2 Facility Design Aspects } & 9-47\end{array}$

9.6.3 Assumptions $9-48$

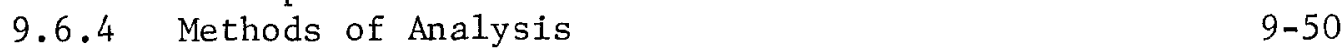

9.6.5 Results 9-53

9.6.6 Conclusions
$9-56$ 
SECTION 10 - MECHANICAL MALFUNCTION ANALYSES

10.1 Loss of Coolant $10-1$

10.1.1 Genera1 10-1

10.1.2 Facility Design Aspects 10-1

10.1.3 Assumptions and Method of Analysis 10-8

10.1.4 Results of Blowdown Analysis 10-9

10.1.5 Results of Core Damage Assessment - Hypothetical Loss-of-Coolant Case 10-17

10.1.6 Coolant Blowdown Calculation 10-18

10.2 Loss of Coolant Flow 10-23

$\begin{array}{lll}10.2 .1 & \text { General } & 10-23\end{array}$

10.2.2 Facility Design Aspects 10-23

10.2.3 Method of Analysis and Selection of Parameters 10-24

10.2.4 Results of Analysis $10-25$

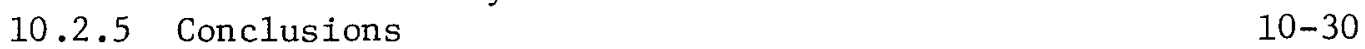

10.3 Fuel Handling 10-31

10.3.1 General 10-31

10.3.2 Fuel Handling Precautions 10-31

10.3.3 Assumptions and Method of Analysis 10-33

10.3 .4 Results 10-33

$\begin{array}{ll}10.4 & \text { Steam Line Break }\end{array}$

10.4.1 General $10-35$

10.4.2 Facility Design Aspects 10-35

10.4.3 Assumptions and Method of Analysis 10-36

10.4.4 Results $10-38$

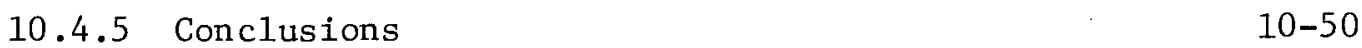

$\begin{array}{ll}10.5 & \text { Steam Generator Tube Rupture 10-57 }\end{array}$

10.5.1 Genera1 10-57

10.5.2 Facility Design Aspects 10-57

10.5.3 Assumptions and Method of Analysis. 10-58

10.5.4 Results $10-60$

10.5.5 Conclusions $\quad 10-60$

$\begin{array}{lll}10.6 & \text { Loss of Load } 10-61\end{array}$

10.6.1 Genera1 10-61

10.6.2 Facility Design Aspects 10-61

10.6.3 Method of Analysis and Selection of Parameters 10-62 


\section{CONTENTS (Continued)}

$\underline{\text { Page }}$

10.6.4 Results

$10-63$

10.6.5 Conclusions

$10-68$

10.7 Waste Gas Accident

$10-69$

SECTION 11 - PLANT BEHAVIOR

11.1 Loss of Power

$11-1$

11.1.1 Genera1

$11-1$

11.1.2 Malfunction in the $220-\mathrm{K}$ or $138-\mathrm{K}$ Switchyard

$11-1$

11.1.3 Malfunction of Plant Equipment

$11-1$

11.2 Earthquakes

$11-6$

11.2.1 General

$11-6$

11.2.2 Aseismic Design Criteria

11.2.3 Classification of Components, Systems

$11-6$ and Structures

11.2.4 Method of Analysis

11.2.5 Examples of Aseismic Design for Critical Components

$11-8$

$11-14$

$11-15$

11.3 Floods

$11-20$

11.3.1 Foothi11 Drainage

$11-20$

11.3.2 Switchyard Drainage

$11-20$

11.3.3 Access Road Drainage

$11-20$

11.3.4 Plant Site Drainage

$11-20$

11.4 Lands lides

$11-22$

11.5 Fire

$11-23$

11.5.1 General

$11-23$

11.5.2 Source of Water

$11-23$

11.5.3 Yard System

11.5.4 Control-Administration Building

$11-23$

11.5.5 Turbine Deck and Auxiliary Bay Structure

$11-24$

$11-24$

11.5.6 Containment Sphere

$11-25$

11.5.7 Warehouse

$11-25$

11.5.8 Ventilating Equipment Room

$11-25$

11.5.9 Reactor Auxiliary Building

SECTION 12 - CHEMICAL REACTIONS

12.1 Chemical Reactions

$12-1$ 
12.1.1 General

$12-1$

12.1.2 Zirconium-Water Reactions 12-1

12.1.3 Parabolic Rate Equation 12-1

12.1.4 Stainless Steel Reactions 12-2

12.1.5 Zirconium-Water Reaction Energy
Release Calculations

12.1.6 Local Transient Calculations 12-3

$\begin{array}{cc}12.1 .7 & \begin{array}{c}\text { Resulting Zirconium-Water Reaction } \\ \text { Energy }\end{array} \\ 12-4\end{array}$

12.1.8 Zirconium-Water Reaction Energy Used
for Containment Sphere Evaluation

$\begin{array}{ll}12.1 .9 \text { Reference } & 12-7\end{array}$

SECTION 13 - FISSION PRODUCT INVENTORY

13.1 Fission Product Release from Fuel 13-1

13.1.1 Introduction 13-1

13.1.2 Core Fission Products 13-1

13.1.3 Reactor Coolant Activity 13-7

13.1.4 Auxiliary Systems Activity 13-8

13.1.5 Spent Fuel Activity 13-11

SECTION 14 - HYPOTHETICAL ACCIDENT

14.1 Hypothetical Accident 14-1

14.1.1 General 14-1

14.1.2 Analysis 14-2

SECTION 15 ENVIRONMENTAL CONSEQUENCES

15.1 Environmental Consequences of Hypothetica1 Accident

15.1.1 General 15-1

15.1.2 Basis for Dose Calculations 15-1

15.1.3 Doses Due to Activity Retained in Containment 15-3

15.1.4 Doses Due to Activity Leaking from the Containment 15-3

15.1.5 Discussion of Results 15-12

15.1.6 References $15-12$

15-2 Environmenta1 Consequences of Other Malfunctions 15-14

15.2.1 Loss of Coolant Accident $15-14$

15.2.2 Waste Gas Accident 15-14 
SECTION 16 - CONCLUSIONS

16.1 Conclusions

APPENDIX A

General Assumptions for Accident Analyses

Reactor Trip

A -1

Hot Channel Conditions

$A-5$

APPENDIX B

Drawing Symbols and Abbreviations

$B-1$ 


\section{ILLUSTRATIONS}

\begin{tabular}{|c|c|c|}
\hline Figure & Title & Page \\
\hline 2.1 & Area Within Fifty Miles of Site & $2-2$ \\
\hline 2.2 & Population Sector Map & $2-4$ \\
\hline 2.3 & Site Arrangement & $2-6$ \\
\hline 2.4 & Plot Plan & $2-7$ \\
\hline 2.5 & Station Profile & $2-8$ \\
\hline 2.6 & Geology and Ground Water Contour Map & $2-10$ \\
\hline 2.7 & Hourly Wind Summary, January, Ream Field Naval Air Station & $2-29$ \\
\hline 2.8 & Hourly Wind Summary, July, Ream Field Naval Air Station & $2-30$ \\
\hline 2.9 & Hourly Wind Speed Summary, July, Lindbergh Field & $2-31$ \\
\hline 2.10 & Hourly Wind Speed Summary, December, Lindbergh Field & $2-32$ \\
\hline 2.11 & Aerial View of the Site & $2-58$ \\
\hline 2.12 & Shallow Subsurface Profile & $2-59$ \\
\hline 2.13 & Fault Map & $2-63$ \\
\hline 2.14 & $\begin{array}{l}\text { Stronger Earthquakes of California and Western Nevada } \\
\text { Through } 1960\end{array}$ & $2-71$ \\
\hline 2.15 & Earthquake Magnitudes, 1932-1942 & $2-72$ \\
\hline 2.16 & Faulting of Sea Floor off Southern California & $2-74$ \\
\hline 2.17 & $\begin{array}{l}\text { Relationship Between Epicenters and Topography of the } \\
\text { Sea Floor off Southern California }\end{array}$ & $2-7$ \\
\hline 2.18 & Earthquake Probabilities Per 100 Years & $2-76$ \\
\hline 2.19 & $\begin{array}{l}\text { Typical Plot of Maximum Particle Velocity in Crystalline } \\
\text { Basement Rock }\end{array}$ & \\
\hline 2.20 & Amplification Spectra & $2-82$ \\
\hline 2.21 & Subsurface Profile & $2-83$ \\
\hline 2.22 & Average Current Frequency Roses & $2-93$ \\
\hline 3.1 & General Arrangement Plans, Sections AA, BB, CC & $3-3$ \\
\hline 3.2 & Genera1 Arrangement Plans, Sections DD, EE & $3-4$ \\
\hline 3.3 & General Arrangement P1an, Elevation 14 Feet & $3-5$ \\
\hline 3.4 & General Arrangement Plan, Elevation 35 Feet, 6 Inches & $3-6$ \\
\hline 3.5 & General Arrangement, Reactor Building Intake Structures & $3-7$ \\
\hline 3.6 & Reactor Core Cross Section & $3-9$ \\
\hline 3.7 & Three-Region Core Loading & $3-10$ \\
\hline 3.8 & Rod Cluster Control Assembly & $3-11$ \\
\hline 3.9 & Fue1 Assemb1y & $3-14$ \\
\hline 3.10 & Rod Assembly and Rod Cluster Control Cross-Section & $3-15$ \\
\hline 3.11 & Fuel Assemb1y, Top and Bottom Views & $3-1$ \\
\hline 3.12 & Partial Section of Spring Clip & $3-18$ \\
\hline 3.13 & Spring Clip Grid and Guide Thimble Assembly & $3-20$ \\
\hline 3.14 & Rod Cluster Control Assembly, Outline Drawing & $3-22$ \\
\hline 3.15 & Reactor Internals & $3-25$ \\
\hline 3.16 & Contro1 Rod Drive Components & $3-30$ \\
\hline 3.17 & Comparison of $\mathrm{W}-3$ Prediction and Uniform Flux Data & $3-42$ \\
\hline 3.18 & W-3 Correlation Probability Distribution Curve & $3-43$ \\
\hline 3.19 & Comparison of W-3 Prediction and Non-Uniform Flux Data & $3-44$ \\
\hline 3.20 & Comparison of the Predicted to Measured DNB Locations & $3-45$ \\
\hline 3.21 & $\begin{array}{l}\text { Comparison of the Predicted to Measured DNB Locations } \\
\text { (Peak Near Exit) }\end{array}$ & $3-46$ \\
\hline
\end{tabular}




\section{ILLUSTRATIONS (Continued)}

Figure

3.22

3.23

3.24

3.25

3.26

3.27

3.28

3.29

3.30

3.31

3.32

3.33

3.34

3.35

3.36

3.37

3.38

3.39

3.40

3.41

3.44

3.45

3.46

3.47

3.48

3.49

3.50

3.51

3.52

3.53

3.54
Title

Page

Comparison of the Predicted to Measured DNB Locations (Peak Near Inlet)

Thermal Conductivity of Uranium Dioxide

$3-57$

Just Critical Boron Concentration Versus Burnup - Hot, Fu11 Power

$3-64$

$3-68$

$3-73$

Position of Rod Bank Versus Percent Power for One Bank

Position of Rod Bank Versus Percent Power for Two Banks, End of Life

Group Position (Fraction Withdrawn) Hot Zero Power, Beginning of Life

Group Position (Fraction Withdrawn) Hot Ful1 Power, Beginning of Life

Average Power Density, No Rods In

Average Power Density Equilibrium Xenon

Average Power Density, One-Third of Cycle

Average Power Density, Two-Thirds of Cycle

Average Power Density, End of Life

First Cycle Power Contour Plot, Beginning of Life

Radial Nuclear Hot Channel Factor Versus Percent Power

for the Maneuvering Band Limits for One Control Group

(17 Rods), Beginning of Life, No Ejected Rods

$3-74$

$3-75$

$3-76$

$3-79$

$3-80$

$3-81$

$3-82$

$3-83$

$3-84$

$3-86$

Nuclear Axial Hot Channel Factor Versus Group Position, Hot No Power

3-87

Nuclear Axial Hot Channel Factor Versus Group Position, Hot Ful1 Power

Total Nuclear Hot Channel Factor Versus Percent Power for the Upper Maneuvering Band Limit for One Control Group (17 Rods), Beginning of Life, No Rods Ejected Moderator Temperature Coefficient Versus Moderator Temperature for Different Boron Concentrations

SELNI Moderator Temperature Coefficient Versus Moderator Temperature at $1600 \mathrm{ppm}$ Boron

SELNI Moderator Temperature Coefficient Versus Boron Concentration

3-88

$3-89$

$3-91$

$3-94$

$3-96$

Comparison of Calculated and Measured Moderator Temperature Coefficient

$3-97$

$3-98$

$3-99$

Power Coefficient Versus Percent Power

$3-101$

Difference in $\mathrm{T}_{\text {eff }}$ Between Measured and Calculated Value

$3-104$

$3-106$

$3-107$

$3-112$

$3-113$

$3-114$ 


\section{ILLUSTRATIONS (Continued)}

Figure

Title

$\underline{\text { Page }}$

3.55

Pressurizer Vesse 1

$3-116$

3.56

Chemical and Volume Control System, Process Flow Diagram

$3-124$

3.57

Xenon Transient and Boron Removal Rate After Startup From Xenon-Free Condition

$3-129$

3.58 Xenon Transient, Boron Removal, and Addition Rates After Load Reduction to Zero Power From Full Power Equilibrium Conditions

$3-130$

3.59 Xenon Transient, Boron Removal, and Addition Rates After Load Reduction to Zero Power, Followed by Restart at Peak Xenon

3.60

3.61

3.62

3.63

3.64

3.65

3.66

3.67

3.68

3.69

3.70

Auxiliary Coolant System, Process Flow Diagram

$3-131$

Sampling System, Process Flow Diagram

$3-143$

Fuel Transfer System, Detail Diagram

$3-152$

Fuel Transfer System, Elevated View

$3-156$

Fuel Manipulator Crane

$3-157$

Radioactive Waste Disposal System, Process Flow Diagram

$3-161$

Reactor Coolant Boron Concentration Versus Operating Time

$3-168$

Liquid Radioactive Waste

$3-170$

$3-171$

Cesium-137 Radioactivity During Core Cycle

$3-176$

Xenon-133 Radioactivity During Core Cycle

$3-177$

Krypton-85 Radioactivity During Core Cycle

$3-184$

4.1

4.2

Drawing Symbol Legend

$4-2$

Heat Balance Diagram

4.3

4.4

4.5

4.6

4.7

4.8

4.9

4.10

4.11

4.12

4.13

4.14

4.15

Steam System

Vents and Drains System

$4-3$

$4-4$

4-5

Circulating Water System

$4-6$

Chemical Feed System.

$4-7$

Turbine System

Feedwater and Condensate System

Sampling System

Compressed Air System

Miscellaneous Water System

Generator Tripping Circuit Block Diagram

$4-8$

$4-9$

$4-10$

$4-11$

$4-12$

4-13

Reactor Tripping Circuit Block Diagram

4-14

Turbine Tripping Circuit Block Diagram

$4-15$

Transmission Systems Diagram

$4-22$

Station Single-Line Diagram

$4-23$

One-Line Diagram, 4160-Volt System 4-37

4.17

4.18

4.19

One-Line Diagram, 480-Volt System

$4-39$

One-Line Diagram

$4-43$

Control Room Area Layout

$4-48$

4.21

Unit Console Instrument Arrangement

$4-49$

4.22

4.23

4.24

4.25

Vertical Board Instrument Arrangement, Left of Console

$4-51$

Vertical Board Instrument Arrangement, Front of Console 4-52

Vertical Board Instrument Arrangement, Right of Console 4-53

Compressed Air System Diagram

$4-57$

4.26

Miscellaneous Water Systems Diagram

$4-58$ 


\section{ILLUSTRATIONS (Continued)}

Figure

5.1

5.2

5.3

5.4

5.5

5.6

5.7

5.8

5.9

5.10

5.11

5.12

5.13

5.14

5.15

5.16

5.17

5.18

5.19

5.20

5.21

5.22

5.23

5.24

6.1

6.2

6.3

6.4

6.5

6.6

6.7

6.8

6.9

6.10

6.11

6.12
Title

$\underline{\text { Page }}$

Safety Injection System, Process Flow Diagram

$5-3$

Feedwater Pump Performance Curves

$5-14$

$5-15$

$5-22$

$5-23$

$5-24$

$5-25$

$5-26$

$5-27$

Radiation Levels During Shutdown Conditions

Radiation Levels During Shutdown Conditions, Sectional

Details

Spent Fuel Transfer Tube Shield

Spent Fuel Transfer Tube Shield Details

Fue1 Storage Building

Maximum Credible Accident

Hypothetical Accident

Type A Sphere Penetration

Type B Sphere Penetration

Type C Sphere Penetration

Type D Sphere Penetration

Type E Sphere Penetration

Type F Sphere Penetration

$5-28$

$5-32$

$5-33$

$5-34$

$5-41$

$5-43$

$5-45$

$5-47$

$5-48$

$5-49$

$5-50$

$5-51$

$5-53$

$5-54$

$5 \mathrm{KV}$ Electrical Power Penetration

$5-56$

Personnel Lock

$6-2$

Source Range Drawer

Functiona1 Block Diagram, Nuclear Instrumentation

Intermediate Range Drawer

Functiona1 Block Diagram, Nuclear Instrumentation Power Range Drawer

Detector Location

Average Temperature Program

Transient Response to Ten Percent Step Load Decrease

From 450 Mwe, Conservative Transient Conditions

Transient Response For Ten Percent Step Load Decrease

From 450 Mwe, Norma1 Transient Conditions

Transient Response for Ten Percent Step Load Increase

From 405 Mwe, Conservative Transient Conditions

Transient Response for Ten Percent Step Load Increase

From 405 Mwe, Normal Transient Conditions

Transient Response For a Reactor Trip and Turbine Trip From 450 Mwe

Transient Response to 5-CPS Step Frequency Decrease

From 60 CPS at 395 Mwe, Conservative Transient

Conditions

Reactor Control System
$6-25$

$6-3$

6-4

6-5

6-16

$6-20$

$6-21$

$6-22$

$6-23$

$6-24$

$6-28$ 


\section{ILLUSTRATIONS (Continued)}

Effect of Pressurizer Spray on Terminal W3 DNB Ratio In Rod Withdrawal Transients

9.11 Terminal W3 DNB Ratio In Rod Wj.thdrawal Transients From Reduced Power Level

Transient Responses For a Fast Rod Withdrawal Accident $\mathrm{T}_{\mathrm{AVG}}=578 \mathrm{~F}$

9.16 Transient Response For Startup of an Inactive Loop Without Full Temperature Decrease

9.17 Transient Response For Startup of an Inactive Loop With Ful1 Temperature Decrease

9.18 Transient Responses For Al1 Three Feedwater Valves Ful1 Open

9.19 Transient Responses For Startup of the Second Feedwater Pump

9.20 Transient Response To 30 Percent Step Load Decrease With Load Limit Tracking

9.21 Response To a Dropped Rod Worth of $-1.25 \times 10^{-3}$ : $\mathrm{k}$ With 


\section{ILLUSTRATIONS (Continued)}

Figure

$\underline{\text { Title }}$

$\underline{\text { Page }}$

9.22 Response To a Dropped Rod Worth of $-2.5 \times 10^{-3} \mathrm{k}$ With Power Cutback to 75 Percent Load, Incremental Control Group Worth $=-2.5 \times 10^{-4} 8 \mathrm{k} /$ inch

$9-46$

10.1 Safety Injection Delivery Characteristics 10-6

10.2 Loss of Coolant - One-Inch (Nominal) Pipe Break 10-10

10.3 Loss of Coolant - Two-Inch (Nomina1) Pipe Break 10-11

10.4 Loss of Coolant - Four-Inch (Nominal) Pipe Break 10-12

10.5 Loss of Coolant - Six-Inch (Nominal) Pipe Break 10-13

10.6 Loss of Coolant - Eight-Inch (Nominal) Pipe Break 10-14

10.7 Loss of Coolant - 10-Inch (Nominal) Pipe Break 10-15

10.8 Loss of Coolant - 27.5-Inch Hypothetical Main Loop Break 10-16

10.9 Reactor Coolant Flow Following Complete Loss of Pump Power 10-26

10.10 Neutron Power Following Complete Loss of Pump Power With Reactor Trip

10.11 Core Heat Flux Following Complete Loss of Pump Power With

$\begin{array}{ll}\text { Reactor Trip } & 10-28\end{array}$

10.12 Minimum DNB Ratio Versus Time F'ollowing Complete Loss of Pump Power With Reactor Trip

10.13 Core Water and Steam Generator Temperatures Related to Time After Break

10.14 Steam Generator Flow and Temperatures Related to Time After Break

10.15 Pressurizer Pressure and Water Volume Related to Time After Break

$10-27$

$10-29$

$10-40$

$10-41$

$10-42$

$10-43$

10.17

10.18

10.19

Reactivity Transients For Both Safety Injection Trains

$10-46$

Reactivity Input to CHIC-KIN Code For "Hypothetical" Clean Water Addition During Steam Break

10.20 Maximum Steam Break "Hypothetical" Clean Water Addition With One Percent Shutdown From Hot Zero Power 10-48

10.21

Radial Power Distribution With One Stuck Rod

$10-48$
$10-49$

10.22 Maximum Steam Break With "Hypothetical" Clean Water Addition

10.23 Changes In Steam Flow and Temperature Related to Time After Break

10.24 Core Water and Steam Generator Temperatures Related to Time After Break

10.25 Reactivity Related to Time After Break

$10 \_47$

$10-51$

$10-52$

$10-53$

10-54

10.26 Accidental Opening of A11 Steam Dump Valves From No Load Condition

10.27 Transient Response For a Full Loss of Load From 450 Mwe Positive Factor

$10-55$

$10-64$

10.28 Transient Response to a Full Loss of Load From 450 Mwe
10.29 Transient Response For a Full Loss of Load From 450 Mwe Negative Factor

$10-65$

10.66

10.30 Negative Transient Response to a Full Loss of Load From 450 Mwe 


\section{ILLUSTRATIONS (Continued)}

Figure

11.1 Main One-Line Diagram

11.2

12.1

12.2

13.1

15.1

15.2

15.3

15.4

15.5

15.6

15.7

15.8

15.9

15.10

15.11

15.12

15.13

15.14

15.15

15.16

15.17

15.18

15.19 Volume
Average Acceleration Response Spectra Normalized For $0.25 \mathrm{~g}$ Ground Acceleration $\underline{\text { Page }}$

$11-2$

$11-7$

$12-5$

$12-8$ Versus Time After Rupture

Heat Generated Versus Time After Rupture

Distribution of Thermal Power as a Function of Core

$13-6$

$15-4$

$15-5$

$15-6$

$15-7$

$15-9$

$15-10$

$15-15$

$15-16$

$15-17$

$15-19$

$15-20$

$15-21$

$15-23$

$15-24$

$15-26$

$15-27$

$15-28$

$15-29$

$15-30$

$A-2$

A-3

$A-4$

A.3 Control Rod Reactivity Insertion Versus Rod Position For Hot Full Power Conditions 
A.4 Reactivity Insertion Versus Time For Reactor Trip, Hot Zero Power Conditions, 120 Percent Reactor Coolant Flow A-6

A.5 Reactivity Insertion Versus Time For Reactor Trip, Hot Ful1 Power Conditions, 120 Percent Reactor Coolant Flow

B.1 Drawing Symbol Legend 


\section{TABLES}

$\underline{\text { Title }}$

$\underline{\text { Page }}$

2.1 Population Distribution, 1960 Census 2-3

2.2 Monthly Temperatures and Precipitation at Camp Pendleton

Surf and Weather Station $2-12$

2.3 Wind Data, Oceanside 2-14

2.4 Wind Summary, Camp Pendleton Surf and Weather Station 2-28

2.5 Frequency of Long Periods of Calms and Light Winds $(0-3 \mathrm{MPH})$

2.6 Frequency of Inversions at Various Heights at Naval Air Station, North Island, San Diego

\section{$2-34$}

$2-36$

2.7 Average Height and Temperature of Inversion Base and Top, and Rate of Temperature Change in Inversion

2.8 Temperatures and Precipitation, San Onofre, December 1964 to June 1965

2.9 Wind Data, San Onofre

2.10 Wind Data, San Clemente

2.11

Areal Distribution of Earthquake Intensity

2.12

Areal Effect of Southern California Earthquakes

2.13

Earthquakes Per 100 Years

2.14

Earthquake Expectancy For a Given Site

Intensity History at Site

2.15

2.16

2.17

Comparison of Site With Average For Southern California

Frequency of Surface Currents at San Onofre

2.18

Frequency of Subsurface Currents at San Onofre

$2-38$

$2-41$

$2-42$

$2-49$

$2-73$

$2-77$

$2-77$

$2-78$

$2-78$

$2-79$

$2-90$

2-91

3.1 Core Mechanical Design Parameters 3-8

3.2

$3-8$
$3-38$

Hydraulic and Thermal Design Parameters

$3-53$

Structural Characteristics

$3-59$

Performance Characteristics

$3-60$

Control Characteristics

3.6

3.7

Kinetic Characteristics

3.8

Reactivity Requirements For Control Rods

$3-61$

$3-62$

$3-66$

Calculated Worth of All Rods and Stuck Rod in Core 3-69

3.9

3.10

3.11

3.12

3.13

Worth of Control Groups

Nuclear Radial Hot Channel Factors

Component Code Requirements

$3-70$

$3-71$

Chemical and Volume Control System Code Requirements 3-140

3.14

Non-Volatile Activity in Coolant and Wastes

$3-173$

Volatile Fission Products

$3-185$

4.1 Equipment Status - Lines in Service, Ties Closed 4-27

4.2 Equipment Status - Villa Park 220-Kv Line Out of Service 4-29

4.3 Equipment Status - One San Luis Rey 138-Kv Line Out of Service

4-31

4.4 Equipment Status - One Tie Bank Out of Service 4-33

5.1 Effects of Second-Order Equipment Failure in Recirculation Subsystem 
TABLES (Continued)

Table

Title

Page

5.2 Safety Injection System - Mechanical and Electrical

System Parameters

$5-11$

6.1 Summary of Tripping Matrix Development 6-12

6.2 In-Core Instrumentation Data

6.3 Radiation Monitoring System Channel Sensitivities and Accuracies

6.4 Detecting Medium Conditions 6-79

9.1 Hot Startup Accident 9-12

10.1 Fission Product Activities Released to Containment Sphere 10-18

10.2 Maximum Waste Gases 10-69

11.1 Damping Factors $11-8$

11.2 Categorization of Reactor Cycle Equipment $11-9$

11.3 Categorization of Structural Equipment $11-11$

11.4 Categorization of Miscellaneous Mechanical Equipment 11-12

11.5 Categroization of Electrical Equipment 11-13

11.6 Summary of Plate Stresses at Grade 11-16

13.1 Direct Radiation Sources Following Fuel Meltdown 13-4

13.2 Radioactive Iodine Sources Following Fuel Meltdown 13-5

13.3 Maximum Fission and Corrosion Froduct Isotopic Inventories of Reactor Coolant For Reference One Percent Fuel Defect Case

13.4 Maximum Fission and Corrosion Froduct Gamma Sources of Reactor Coolant For Reference: One Percent Fuel Defect Case

13.5 Direct Gamma Sources Contained in Volume Control Tank For Reference One Percent Fuel Defect Case

13.6 Direct Gamma Sources and Isotopic Inventory of Waste Gas Decay Tanks For a Reference One Percent Fuel Defect Case

13.7 Direct Gamma Sources and Isotopic Inventory For the Spent Fuel Gap Activity 90 Hours Following Shutdown

14.1 Theoretical Complete Core Meltdown Fission Product Sources 14-3

15.1 Distance Summary Table 15-13

A.1 Assumed Time Delays A-1 


\section{SECTION 7}

ADMINISTRATION

\subsection{ORGANIZATION AND TRAINING}

\subsubsection{GENERAL}

The San Onofre Nuclear Generating Station was designed, constructed and will be operated with the basic goal of producing electric power in the most economic manner consistent with safety to the public and plant personnel.

Since no post-operational research or experimental work is anticipated, station operations will require a prudent minimum of personnel. However, experience and data derived from operation will be of significant value to the national program of nuclear power development.

In accordance with the agreement between the Southern California Edison Company, the Bechtel Corporation and the Westinghouse Electric Corporation, Westinghouse will have the responsibility of supervising the starting up and initial phases of operation, including acceptance testing, until the station is available for operation. Edison will be responsible for supplying the appropriate AEC licensed personnel for operating the station. Bechtel will provide engineering and construction assistance.

Upon acceptance of the station for commercial operation, Edison will have the full responsibility for operation and maintenance. The organization chart shown in Figure 7.1 has been prepared for the full time employees of the station. In addition to the guard force, security will consist of fencing restricted areas and utilizing the operating force that will be on duty at all times. Routine maintenance will be performed by the maintenance force. Major maintenance of equipment will be accomplished by maintenance crews from the Edison System or contracted as circumstances dictate.

The normal operating shift complement will consist of four men; a Watch Engineer who is in charge, a Control operator, an Assistant Control Operator and a Plant Equipment Operator. Supervision, technical support, administration, maintenance and other staff personnel make up a total of 47 people working full time at the station site. Additional support personnel such as clerical, technical and maintenance personnel are available from the Edison System.

Normal duties for the station personnel will involve operation and maintenance of the station including the electrical switchyard, receiving new fuel, shipping spent fuel, monitoring station performance, chemical and radiation monitoring and control, record keeping, reordering expended supplies and housekeeping. Edison has entered into an interim agreement with Westinghouse for fuel service for the unit.

Operation of the entire station is under the supervision and control of the Station Chief. Reporting directly to the Station Chief are the Supervisor 


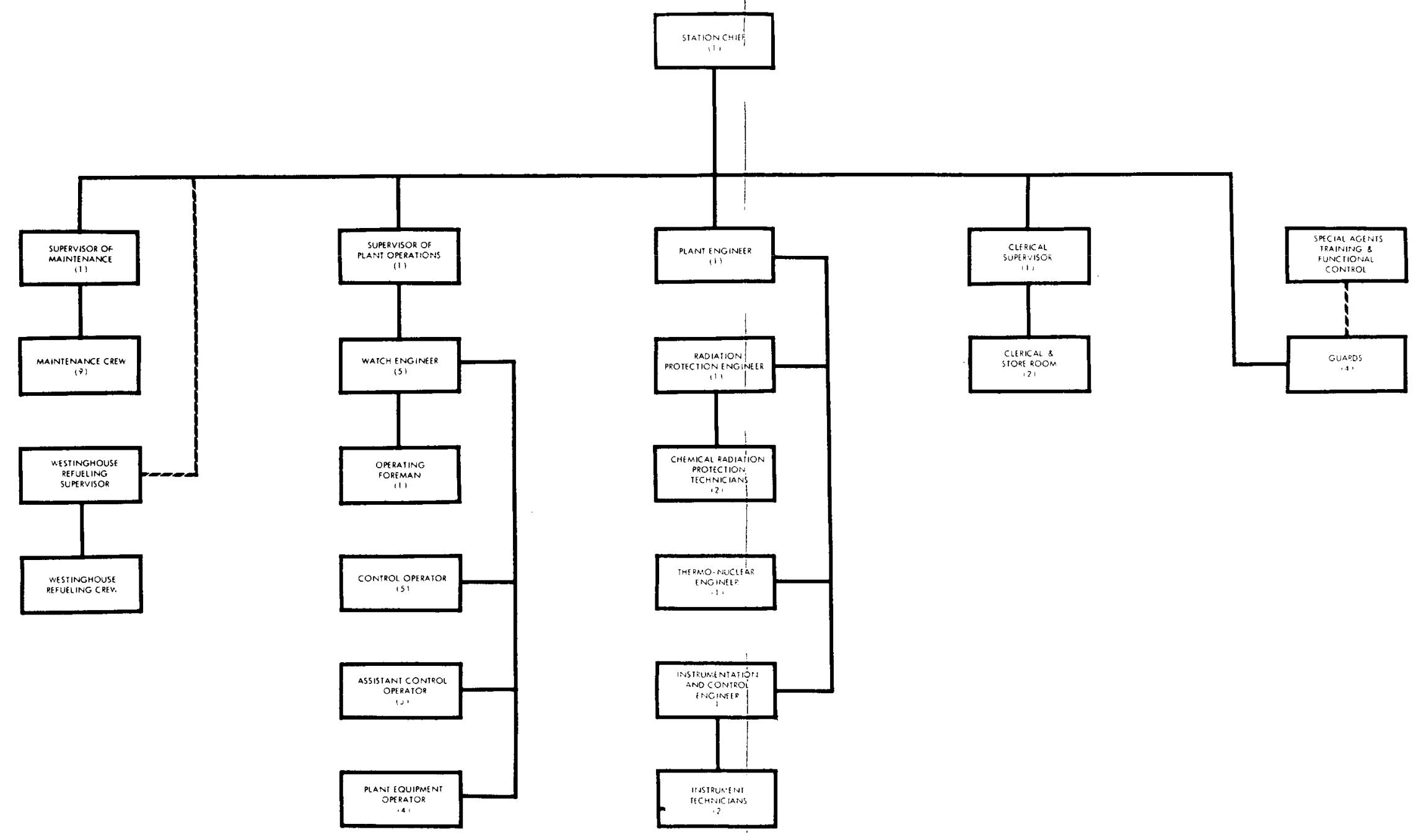

FIGURE 7.1 ORGANIZATION CHART 


\subsubsection{GENERAL (Continued)}

of Plant Operation, who is responsible for the station operation and security, the Plant Engineer, who is responsible for technical services, instrumentation, health physics and water chemistry, the Maintenance Supervisor who is responsible for maintenance and the Clerical Supervisor who is responsible for administration functions and storekeeping.

\subsubsection{OPERATION}

Reporting directly to the Supervisor of Plant Operations are the Watch Engineers who will supervise and control full time operation of the operating crew. The normal operating forces will consist of a control operator, an assistant control operator and an equipment operator. Additional operating personnel will be available to supplement a shift when unusual operating conditions exist. The normal duties of a shift will be to start up and shut down the entire station, monitor and control normal operation and load changes, operate all of the various auxiliary systems, maintain operating records and enforce plant security. The operating crew will have experience and training in both conventional and nuclear power plants. Personnel who direct the activities of the operators will be licensed as AEC Senior Reactor Operators.

\subsubsection{PLANT TECHNICAL STAFF}

Reporting to the Plant Engineer are the Thermo-Nuclear Engineer, the Chemist-Radiation Protection Engineer and the Instrument Engineer. These supervisors along with their respective technicians comprise the station technical staff. Additional technical support will be available from the Edison Engineering Department, the Edison Steam Division Technical Staff Westinghouse and consultants in specified fields.

The Thermo-Nuclear Engineer will be responsible for the following:

a) Performance Monitoring - Engineering tests on all turbine cycle equipment to verify safe and efficient operation.

b) Core Fueling and Physics Tests - Initial and subsequent core fueling and physics tests in cooperation with Westinghouse personnel.

c) Thermal and Nuclear Operation of the Reactor Core - Monitoring and testing in regard to thermal and nuclear operation of the core to verify safe and efficient operation of the reactor cycle.

The Chemist-Radiation Protection Engineer is responsible for the following:

a) Chemical Treatment - Chemical treatment of water and all solid, liquid and gaseous waste. Seeing that all waste shipment or release of material from the station complies with federal, state or local regulations. 


\subsubsection{PLANT TECHNICAL STAFF (Continued)}

b) Radiation Protection - Seeing that policies relating to personnel radiation exposures within the station are established and enforced and records of personnel exposures maintained, appropriate biological samples are taken at established intervals, appropriate radiation and contamination surveys are made to verify safe radiation levels and working conditions throughout the plant site, and training technicians to handle all phases of the job. Additional technicians will be available from the Steam Division Chemical Section during special peak periods if required.

The Instrumentation Engineer's staff will consist of two instrument technicians. Additional instrument and test technicians will be available from the Edison system. The Instrument Engineer is responsible for the following:

a) Station Instrumentation - Seeing that station electrical protective and metering equipment and all reactor and turbine cycle control systems and instrumentation, including stationary and portable radiation monitoring and laboratory equipment are maintained, calibrated and repaired as required.

b) Instrumentation Records - Keeping appropriate records to verify the proper working order of a 11 instrumentation.

\subsubsection{STATION MAINTENANCE}

The Maintenance Supervisor will be responsible for all mechanical and electrical maintenance within the station. The maintenance crew, consisting of one electrician, one machinist, one welder, one boiler and condenser mechanic and three helpers, will handle the normal preventative maintenance and housekeeping duties. Additional help will be available from the Edison Steam Division Maintenance forces or may be obtained from a contractor. The major maintenance work load is expected during refueling. Under an agreement for fuel service, Westinghouse will furnish additional personnel for the refueling period.

\subsubsection{PERSONNEL PREVIOUS TRAINING AND BACKGROUND}

The following personnel will initially be employed in the station:

a) Station Chief - Graduate Mechanical Engineer 1947. Thirteen years experience in engineering and administrative positions in conventional power plants. Training since August 1964 in the nuclear field.

b) Supervisor of Plant Operation - Graduate Mechanical Engineer 1951. Registered Professional Engineer. Fourteen years experience in engineering and administrative positions in conventional power plants. Training since August 1964 in the nuclear reactor field.

c) Plant Engineer - Graduate Mechanical Engineer 1959. Six years experience in engineering and administrative positions in conventional 


\subsubsection{PERSONNEL PREVIOUS TRAINING AND BACKGROUND (Continued)}

power plants. Training since November 1964 in the nuclear reactor field. Will be certified at Saxton Nuclear Power Plant.

d) Maintenance Supervisor - Several years of formal technical training beyond high school including courses leading to a certificate in nuclear engineering at UCLA. Successfully passed the Engineer in Training Examination in the State of California. Employed in industrial maintenance since 1938 with several years as a supervisor. Training since February 1965 in the nuclear field.

e) Watch Engineers - Five have from 10 to 20 years experience in conventional power plants and one has several years experience as a reactor operator in the U. S. Navy and as a shift supervisor at Atomics International Santa Susana site. Five have been in training since November 1964 in the nuclear reactor field.

f) Thermo Nuclear Engineer - Graduate in Mechanical Engineering and Business Administration 1962. Three years experience as an engineer in conventional power plants. Training since July 1965 in the nuclear reactor field.

g) Chemist-Radiation Protection Engineer - Graduate Chemist 1957. Eight years experience as a chemist in the conventional power plant field including several years as a supervisor. Training since March 1965 in the nuclear power plant field.

h) Instrument Engineer - Several years formal technical training beyond high school. Employed for several years in conventional power plants in the instrumentation field as a journeyman and as a supervisor. Training since March 1965 in the nuclear power plant field.

i) Control Operators - All five have several years experience in conventional power plants. Training since March 1965 in the nuclear power plant field.

j) Assistant Control Operators - All six have several years experience in conventional power plants. Training since July 1965 in the nuclear power plant field.

\subsubsection{NUCLEAR TRAINING PROGRAM}

\section{Station Personne1}

The station supervisors and operating personnel were obtained from Southern California Edison Company fossil-fired steam generating stations. Those selected for the nuclear station have had years of experience with corresponding positions in Edison generating stations and have the background to learn nuclear technology.

In order to realize full benefit from the Westinghouse Reactor Operator Training Program, a six week review course was initiated for the operating 


\subsubsection{NUCLEAR TRAINING PROGRAM (Continued)}

supervisors and control room personnel prior to entering the formal training program. The subject matter consisted of an intensive and concentrated review of algebra, logarithms, exponentionals, slide rule, scientific notation, general physics and basic nuclear physics.

In August of 1964, approximately two years before scheduled fuel loading, the Station Chief and the Supervisor of Plant Operation attended the three month Reactor Operators Training Program at the Westinghouse Reactor Evaluation Center, Waltz Mill, Pennsylvania. The training program included classroom lectures on atomic, nuclear and reactor physics, reactor control theory and instrumentation, reactor safety, radiation protection, AEC regulations, WREC operating procedures and the reactor critical experimental system. Practical application of classroom lectures was obtained by performing reactor fuel loading operations and experiments followed by individual reactor operations and instructions, evaluation of experiments, radiation protection and health physics procedures and study. The last week of the program included a review of the entire course, a written examination and a critical experimental system reactor operation examination.

The Station Chief and the Supervisor of Plant Operation, under Westinghouse supervision, began reactor operator training at the Saxton Nuclear Experimental Corporation, Saxton, Pennsylvania, in November of 1964. This training was administered in two phases. The first phase consisted of acquiring knowledge of the reactor systems, technical specifications, hazards analysis and operating and emergency procedures. Radiation and protection training was of approximately four months duration.

The second phase of the training was allotted to learning the duties and procedures of the facility management, operation and maintenance. The trainees spent a week with each of the following groups: shift supervisor, electrica1 and instrument maintenance, radiation protection, chemistry and water analysis, and plant maintenance. In addition, one week was spent in discussion and in becoming familiar with Saxton personnel and their positions. During the last week of April 1965, as determined by examinations administered by the AEC Division of Reactor Licensing, these two trainees met the requirements for an operator as set forth in 10CFR55.

The Station Chief, Supervisor of Plant Operation and Plant Engineer attended a seven week lecture and discussion series in May and June of 1965 in which the Westinghouse Atomic Power Division design engineers reviewed the design of reactor components, systems, controls and instrumentation systems and the expected operating characteristics of the San Onofre reactor cycle.

The Station Chief, Supervisor of Plant Cperation, Plant Engineer, Supervisor of Plant Maintenance and Chemist-Radiation Protection Engineer were present at the Yankee Atomic Electric Company facility during Core IV-V refueling in August, 1965.

The Plant Engineer, after conducting the review training course on the west coast in September and October, entered the Reactor Operator Training Program at the Westinghouse Reactor Evaluation Center, Wa1tz Mi11, Pennsylvania in November, 1964. 


\subsubsection{NUCLEAR TRAINING PROGRAM (Continued)}

In February, 1965, the Plant Engineer was assigned for a three-month period to work with the Westinghouse Atomic Power Division nuclear reactor analysis group. This training consisted of learning the application, use and analysis of in-core instrumentation, the application and use of reactor computer codes, determination of hot channel factors, fuel burn-up calculations and reactivity follow computations.

In May and June 1965, the Plant Engineer attended the Westinghouse Atomic Power Division training lectures with the Station Chief and Supervisor of Plant Operation.

Training for reactor operator certification of the Plant Engineer started in early July of 1965 at the Saxton Nuclear Experimental Corporation Facility in Saxton, Pennsylvania. This training was similar to the Station Chief and Supervisor of Plant Operation's program with the exception of the administrative training for which experimental work with the on-site Westinghouse Nuclear Engineer was substituted. An AEC reactor operator examination for Saxton will be given to the Plant Engineer in December, 1965.

The Watch Engineers began training in September of 1964 in the review training course on the west coast. Starting in November, 1964 they entered into the three month Reactor Operator Training Program at the Westinghouse Reactor Evaluation Center, Waltz Mill, Pennisylvania.

The Watch Engineers reported to the Saxton Nuclear Experimental Corporation facility for reactor operator training in February, 1965. This training program was similar to that administered to the Station Chief and Supervisor of Plant Operation with the exception of the administrative portion. However, in addition to the operation of the facility, ten supplementary lectures were given for review of reactor physics and engineering. The Watch Engineers successfully completed this portion of the training program as determined by an examination administered by the AEC Division of Reactor Licensing, met the requirements for an operator as set forth in 1OCFR, Part 55 .

Commencing in mid-July, 1965, the same lecture series was conducted at Westinghouse Atomic Power Division for the Watch Engineers and two staff engineers as was given previously to the Station Chief, et al. Specialized training was given to the Plant Engineer, whose program was previously reviewed, Supervisor of Plant Maintenance, Chemist-Radiation Protection Engineer, Instrument Maintenance Engineer and the Thermo-Nuclear Engineer. These training programs ran concurrent with the overall reactor operator training program and are described below.

The Supervisor of Plant Maintenance began training at the Saxton Nuclear Experimental Corporation facility on February 1, 1965. His training was in conjunction with the installation of the Saxton reactor supercritical 1oop and consisted of the fabrication of the filter package assembly, the fabrication and installation of the supercritical loop piping in the storage well area of the containment vessel, the examination of radiographs, and cleaning, storing and handling methods as applied to materials related to nuclear service. Experience in the problems of working in radioactive 


\subsubsection{NUCLEAR TRAINING PROGRAM (Contirued)}

contaminated areas, application of work clothing and personnel exposure to radiation was acquired.

Additional training was obtained at the Saxton site when the trainee was assigned to the Supervisor of Reactor Plant Service in March and again in June of 1965. This training included problems common to reactor plant maintenance, work methods, approach and solutions.

Upon completion of the lecture series at the Westinghouse Reactor Evaluation Center in late March and Apri1, 1965, the trainee was assigned to the Westinghouse Waltz Mill site for further training. This portion of the program extended through May to mid-June of 1965. The program consisted of the use and calibration of portable radiation survey and monitoring equipment, smear techniques and counting room procedures, loading and shipping of spent fuel casks, application and use of film badges and dosimeters, decontamination and clean-up methods, the use of proper clothing, and handling highly contaminated materials. The trainee also attended lectures at WAPD in those areas where such information would be useful at San Onofre and related to the trainee's job assignment.

Additional training was obtained by the trainee's presence at the Yankee Atomic Electric Company Core IV-V refueling in August of 1965.

The Chemist-Radiation Protection Engineer began training in February, 1965 with the review course given on the West: Coast. In March and April the trainee attended the lecture series and reactor fuel loading training at the Westinghouse Reactor Evaluation Center.

On April 26, 1965 the Chemist-Radiation Protection Engineer attended courses at the Taft Sanitary Engineering Center for instruction in Basic Radiological Health and in Occupational Radiation Protection.

Upon completion of the above described course, the Chemist-Radiation Protection Engineer entered into training during late May and June, 1965 at the Westinghouse facility at Waltz Mill, Pennsylvania. This training program consisted of learning the use and calibration of portable radiation survey and monitoring equipment, smear techniques and counting room procedures, radiochemistry, use of a 400 channel gana radiation spectrometer, application and use of film badges and dosimeters. During this training he attended lectures at WAPD in those areas related to radiation monitoring and shielding. In July, 1965 additional. field training was obtained at the Saxton Nuclear Experimental Corporation in cooperation with their Radiation Protection Engineer. Further experience was gained at the Yankee Atomic Electric Company refueling of Core IV-V in August, 1965.

The Instrument Engineer began training in February, 1965 with the review course given on the west coast. In March and April the trainee attended the lecture series and reactor fuel loading training at the Westinghouse Reactor Evaluation Center.

In May, 1965, the Instrument Engineer moved to the Carolinas-Virginia Test 


\subsubsection{NUCLEAR TRAINING PROGRAM (Continued)}

Reactor at Parr, South Carolina for a four month training program. This training was to acquire knowledge and experience in reactor control system components similar to those of San Onofre, nuclear instrumentation, portable radiation survey and monitoring equipment, counting room equipment and radiation protection measures. During the first two weeks of June the trainee attended lectures at the Westinghouse Atomic Power Division related to contro1, radiation monitoring and nuclear instrumentation at San Onofre.

The trainee attended and participated in the initial tests of the San Onofre nuclear instrumentation during this period.

Additional training and experience was acquired by the trainee's participation in the on-site installation, testing and calibration of the nuclear instrumentation and reactor control system.

The Thermo-Nuclear Engineer entered training in mid-July by attending the WAPD lecture series with the Watch Engineers. Following the lecture series he began three months training at the Sexton Experimental Nuclear Corporation facility. Here he trained in the Westinghouse Experimental Program at Saxton under the supervision of the on-site Nuclear Engineer. This training consisted of learning experimental techniques, reactor core physics tests, flux wire systems, hot channel factor computations, reactivity calculations and tests, and fuel burn-up calculations.

The control operators began training in February, 1965, with the review course detailed previously. In mid-March they entered the three month Reactor Operator Training Program at Waltz Mil1, Pennsylvania. Beginning in July of 1965, the control operators participated in a three month training program at the Saxton Nuclear Experjmental Corporation, Saxton, Pennsylvania. The purpose of this training was to provide familiarity in the operation of reactor systems and controls, operating and emergency procedures and radiation protection. In addition ten supplementary lectures were presented on reactor physics and engineering. After a three month interval, the assistant control operators received the same training as the control operators. This training was conducted for the purpose of future AEC licensing following the San Onofre reactor core physics tests.

On-site training began in October, 1965, approximately one year in advance of scheduled fuel loading. This training is being conducted by the operating supervisors who will be preparing for a Senior Cold Reactor Operator's license for San Onofre in cooperation with the reactor manufacturer's startup engineers. Initially participating in this three month training will be all on-site station supervisors and the control operators. Beginning in January, 1966, the training program will be repeated with the Plant Engineer, Thermo-Nuclear Engineer, assistant control operators, plant equipment operators, and technicians assigned to the Station at this time, in attendance. The on-site training program is directed towards acquiring facility knowledge and familiarity for AEC Senior Reactor Operator 1icensing of the operating supervisors and selected staff members prior to core loading. Control room operators will be qualified to take the AEC Reactor Operator licensing examination after completion of the core physics tests. 


\subsubsection{NUCLEAR TRAINING PROGRAM (Continued)}

On-the-job-training for replacement personnel and up-grading for training purposes will be conducted under established company policies. In addition, a program of continued training will be established for all operators.

In order to safeguard the public and plant personnel, practice emergency drills will be conducted in accordance with an emergency plan. Such practice drills will be coordinated with local public authorities.

\section{Staff Support Personne1}

Personnel skilled in the areas of radiation protection (health physics) and nuclear engineering are required so that competent staff support is available for design, construction, and startup of the plant. In order to provide this competency, a comprehensive training program was developed.

The facilities selected provided the most prudent program for obtaining the specific training objectives. These facilities and their purposes are listed below:

\section{Nuclear Engineering}

- Oak Ridge - 12-month course at the Oak Ridge School of Reactor Technology.

- Westinghouse Atomic Power Division - 2-month program on the design of the San Onofre plant systems.

- University of Michigan - 1-month program of intensive instruction on nuclear engineering fundamentals.

- University of California - 1-week refresher course on nuclear engineering principles.

- Los Angeles DWP - 1 year spent with BWR study team that provided an excellent background in reactor plant design.

- General Atomic - 15-month assignment on HTGR reactor development project providing information in impurity removal and general plant design.

- University of California - 9-week intensive course, attended by engineers in nuclear engineering at a graduate level.

- Atomics Internationa1 - 12-month assignment on GBSR reactor development project concerning overall plant design.

\section{Radiation Protection}

- University of California - 12-month program, both academic and field work, that provides a comprehensive background in radiation protection. 


\subsubsection{NUCLEAR TRAINING PROGRAM (Continued)}

The following numbers of personnel received training at these facilities: one at Oak Ridge; one at Westinghouse's Atomic Power Division; three at the University of Michigan; six at the University of California; two worked with the Los Angeles Department of Water and Power study team; two worked with General Atomic; one worked with Atomics International; one attended a 12-month program at the University of California that included three months of field work at the National Reactor Test Station in Idaho; and one attended a nine-week intensive course in nuclear engineering at the University of California.

These personnel, plus those with previous experience in the nuclear field, and the engineering staff which has actively participated in the design and construction of the facility, provide a staff that is available to assist station personnel.

\subsubsection{OPERATING PROCEDURES}

Plant operation will be conducted in accordance with accepted standards of safe operating procedures and written jnstructions. These instructions fall in three classifications; operating, emergency and maintenance.

Operating instructions describe routine operation of reactor-related auxiliary systems, reactor startup, operation at power, and reactor shutdown. Each instruction lists procedures for the particular operations involved.

Emergency instructions cover the range of plant troubles. These instructions describe the conditions prevalent, probable observable symptoms, immediate corrective action, long term corrective measures and notification of public authorities for the safeguarding of the public and plant personnel. Detailed emergency instructions appear in a separate document.

Maintenance instructions, which will supplement the existing accident prevention rules and safe work practices, incorporate procedures, conditions and instructions in the maintenance of equipment handling or containing radioactive fluids or materials.

All written procedures and modifications will be prepared by qualified personnel who are thoroughly familiar with the reactor and its operation. Each procedure or revision will be reviewed periodically for possible updating or revision to insure that they are current and in keeping with the physical status of the plant.

\subsubsection{RECORDS}

Records related to the plant will be maintained pursuant to the requirements of the technical specifications. Principal records retained are daily logs of operation, tests, inspections and measurements. In accordance with Edison policy, operating charts for the first year's operation will be permanently stored while later such records will be retained on a three year rotational basis. 


\subsection{TESTING AND INITIAL OPERATION}

\subsubsection{GENERAL}

The initial testing and operation of the plant mechanical and electrical systems prior to full power operation of the plant is divided into three phases:

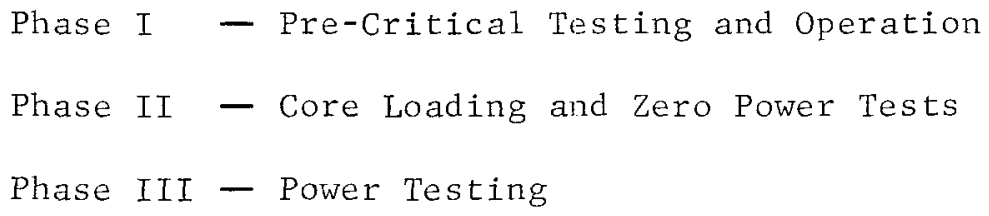

The purpose of this program is to perform necessary testing and systems operations to demonstrate that the equipment has been installed and will operate in accordance with the design requirements (Phase I); to provide safe procedures for initial fueling and determination of those core parameters significant to the core design in the zero power tests (Phase II); and to bring the plant to its rated capacity in a safe and orderly fashion and thereby complete the overall plant systems tests (Phase III).

Systems operations throughout the program will be conducted by the plant operators following startup procedures to be prepared by Westinghouse and Bechtel and reviewed by Edison. These procedures will include a delineation of administrative procedures and test responsibility, equipment clearance procedures, and an overall sequence of startup operations.

Procedures stating the test purpose, conditions, precautions, and limitations will be prepared for each test phase. A description of pre-critical tests to be performed on specified safeguard systems is contained in the paragraph that describes the pertinent system.

\subsubsection{PHASE I - PRE-CRITICAL TESTING AND OPERATION}

The program includes those tests, adjustments, calibrations and system operations necessary to assure that initial fuel loading and subsequent power operation can be safely undertaken. Where feasible, plant mechanical systems are ultimately operated at full load conditions of pressure, temperature and flow prior to core loading. A general description of tests to be performed prior to initial reactor fueling to indicate the nature and scope of the program is contained in the following paragraphs.

\section{Electrical Testing}

Al1 electrical protective relay systems will be tested for correct operation and calibration. All automatic bus transfer switching systems will be tested for correct operation, sequencing and dynamic response with related hydraulic and pneumatic systems. 


\subsubsection{PHASE I - PRE-CRITICAL TESTING AIND OPERATION (Continued) \\ Instrument Calibration}

Instrumentation and control devices will be checked to assure their accuracy. Primary sensing elements, transducers, transmitters, receivers, recorders and indicators will be thoroughly inspected and adjusted for accuracy of their set point characteristics. Interconnecting piping and wiring will be checked for continuity and functional requirements. Each device will then be tested in accordance with established test procedures. Limit switches used for initiating indicating lights, alarms and interlock functions will be checked under actual or simulated operating conditions.

Control devices will be exercised to assure proper operation with the required accuracy and response characteristics. Set points for devices will be checked and adjusted to their operating values.

Each individual circuit of the reactor and turbine protection systems will be tested to verify that appropriate signals initiate reactor and turbine trips. As a signal level corresponding to the particular condition is reached, trip or cutback functions will annunciate as provided for the particular channel under test.

Reactor and Turbine Cycle Cleaning and Flushing

Plant cleaning and flushing operations will be performed to prepare fluid systems for power operation. Procedures which will include proper water chemistry and additives will be established to achieve and maintain the cleanliness of the systems.

\section{Reactor and Turbine Cycle Hydrostatic Testing}

Hydrostatic testing of all plant auxiliary systems will be conducted in accordance with the applicable state and Code requirements. These tests are normally performed as soon as the piping systems are completed, flushed, and ready for testing. This testing supplements the shop hydrostatic testing of the various plant components. Where applicable, surveillance of equipment temperatures is maintained, in conjunction with other special precautions.

\section{Reactor Cycle Hot Functional Tests}

The Reactor Coolant System will be tested to check heatup and cooldown procedures (using pump heat); to demonstrate satisfactory performance of components prior to installation of the core; to verify proper operation of instrumentation, controllers and alarms; and to provide operating conditions for checkout of auxiliary systems.

The Chemical and Volume Control System will be tested to determine that water can be charged at rated flow against normal Reactor Coolant System pressure; to check letdown flow against design rate for each pressure reduction station; to determine the response of the system to changes in pressurizer level; to check procedures and components used in boric acid batching and transfer operations; to check operation of the reactor makeup 


\subsubsection{PHASE I - PRE-CRITICAL TESTING AND OPERATION (Continued)}

contro1; to check operation of the excess letdown and seal water flowpath; and to verify proper operation of instrumentation, controllers and alarms.

The Sampling System will be tested to demonstrate that a specified quantity of representative fluid can be obtained safely and at design conditions from each sampling point.

The Auxiliary Coolant System will be tested to evaluate its ability to remove heat from reactor coolant; to verify component cooling flow to all components; and to verify proper operation of instrumentation, controllers and alarms.

The Safety Injection System will be tested to check the time required to actuate the system after a safety injection signal is received; to check that pumps and motor operated valves are properly sequenced; and to verify proper operation of instrumentation, controllers and alarms.

The Radioactive Waste Disposal System will be tested to verify satisfactory flow characteristics through the equipment; to demonstrate satisfactory performance of pumps and instruments; to check for leak-tightness of piping and equipment; and to verify proper operation of alarms.

The ventilation system will be tested to adjust proper flow characteristics of ducts and equipment; to demonstrate satisfactory performance of fans, filters, and coolers; and to verify proper operation of instruments and alarms.

\subsubsection{PHASE II - INITIAL CORE LOADING AND ZERO POWER TESTS}

\section{Core Loading}

After the Phase I tests have been satisfactorily completed, plant conditions will be established under which the initial nuclear fuel loading can be accomplished. Water in the reactor vessel will be borated to maintain the effective multiplication constant of the core at or below 0.90 at all stages of the core loading. The Reactor Coolant System will be isolated and applicable tagging and administrative procedures will be used to prevent unauthorized change in the boron concentration. The boric acid tank will be filled with concentrated boric acid solution and the residual heat removal system will be in service and available to provide moderator mixing and temperature control, if required. A detailed preloading checkoff 1 ist will be followed to insure that all systems, equipment and conditions affecting the loading operation are met. Periodically, the checkoff list will be reviewed to insure that systems and equipment continue to meet requirements of the core loading operation.

The core loading sequence will follow a step procedure to insure at each loading step that:

a) Fuel assemblies of the correct enrichments are installed in the proper locations. 


\subsubsection{PHASE II - INITIAL CORE LOADING AND ZERO POWER TESTS (Continued)}

b) Rod cluster control assemblies are inserted into the proper fuel assemblies prior to loading the assemblies into the core.

c) Neutron sources and neutron detectors are properly located in the core during fueling. Radiation monitoring will be provided at the core loading stations during fuel handling and core loading operations.

\section{Zero Power Reactor Physics Measurements}

After the core has been loaded according to the prescribed procedure and the reactor vessel head assembled on the vessel, zero power physics measurements will be made to obtain experimental values of reactivity parameters and coefficients important to plant operation.

\section{Initial Criticality}

With the plant at ambient condition, the pretest checkoff 1 ist will be completed and the core brought from 10 percent shutdown to criticality by first withdrawing all control rods and then subsequently reducing the boron concentration in the coolant by dilution until a small startup rate is established. Source range counts will be monitored and recorded, and the inverse multiplication ratio will be computed and plotted as criticality is approached. Ambient tests will then be completed and the plant brought up to no-load temperature and pressure and a second series of zero power tests will be initiated.

\section{Zero Power Reactivity Coefficient Evaluation}

After initial criticality has been established and proper operation of the nuclear instrumentation and special test equipment has been verified, a sequence of zero power reactor physics measurements will be undertaken. The sequence of tests will consist of evaluations of temperative and pressure coefficients and of integral control rod worths. These tests will cover a series of prescribed control rod configurations with intervening measurements of differential control rod worths and boron worth during boron dilution or boron injection. As the successive configurations are established, the measurement techniques to be used will be:

a) Dynamic Temperature Coefficient Measurement - Differential moderator coefficient measurements will be rnade by continuously increasing or decreasing the moderator average temperature and observing the resultant change in core reactivity.

b) Dynamic Pressure Coefficient Measurement - Differential moderator pressure coefficient measurements will be made by continuously increasing or decreasing the moderator pressure and observing the resultant change in core reactivity.

c) Control Rod Worth Measurements by Rod Drop at Zero Power - Integral control rod worth measurements will be made at zero power by dropping 


\subsubsection{PHASE II - INITIAL CORE LOADING AND ZERO POWER TESTS (Continued)}

one or more control rods from a just critical configuration and determining the resultant change in core reactivity by observing the transient flux level response to the negative reactivity insertion.

d) Dynamic Control Rod Worth Measurements - Control rod differential worth measurements will be made by monotonically withdrawing or inserting selected control rods or groups of rods and observing the resultant change in core reactivity.

e) Dynamic Boron Worth Measurements -- Differential boron worth measurements will be made by monotonically increasing or decreasing main coolant boron concentration and observing the resultant change in core reactivity.

\subsubsection{PHASE III - POWER TESTING}

The plant will be brought to full power in discrete steps. A series of overall plant and core tests will be performed to determine the plant characteristics and to establish that the next succeeding power level can be safely attained. When the plant has attained full power operation, a series of measurements will be made to establish the operational characteristics of the plant and the core.

\section{Electrical Trip Testing}

Electrical tripping relays that are initiated by plant on-power malfunctions will be retested and the consequent trip sequence rechecked under operating conditions for correct operation and sequence.

\section{Turbine Trip Testing}

The turbine protection system will be checked to confirm that the appropriate initiation will either trip the turbine through the main trip solenoid or will mechanically trip the turbine. As the various set-points or status conditions are reached, the trip or runback functions will be verified.

\section{Elevated Power Reactivity Coefficient Evaluation}

During the approach to full power and during initial operation at power a sequence of reactor physics measurements will be carried out to experimentally determine power and temperature coefficients and power defects at various power levels, differential control rod worth and boron worths during boron dilutions, and xenon worth and depletion rate during initial operation. Measurement techniques are:

a) Dynamic Differential Power Coefficient - Differential power coefficient measurements are to be made at elevated power over a limited range in power level by initiating a small power level change, allowing moderator average temperature to compensate for the change in core reactivity with control rods fixed and, finally, adjusting control rod position to return moderator average temperature to its injtial value. The change in core 


\subsubsection{PHASE III - POWER TESTING (Continued)}

reactivity associated with the compensating control rod motion, is to be related to the net change in power level.

b) Dynamic Power Defect Measurements - The change in reactivity defect associated with a relatively large change in power level is to be measured by adjusting control rod positions during a ramp change in power level to maintain moderator average temperature at the prescribed value and by observing the compensating change in core reactivity due to control rod movement as indicated by the reactivity conputer.

c) Dynamic Temperature Coefficient Measurements at Elevated Power Differential moderator temperature coefficient measurements are to be made at elevated power over a limited temperature range by monotonically withdrawing or inserting control rods and observing both the resultant change in core reactivity at each step as indicated by a reactivity computer and the corresponding reactivity-compensating change in moderator average temperature at constant power level.

d) Dynamic Contro1 Rod Worth Measurements - Control rod differential worth measurements are to be made at elevated power and by initiating a transient change in boron concentration in the coolant by adjusting control rod position during the transient to maintain moderator average temperature and power level essentially constant, and by observing the compensating change in core reactivity due to control rod movement as indicated by the reactivity computer.

e) Dynamic Boron Worth Measurements -- Differential boron worth measurements are to be made at elevated power by monotonically increasing or decreasing main coolant boron concentration. Compensation for the reactivity effect of the boron concentration change will be made by withdrawing or inserting, respectively, control rods to maintain moderator average temperature and power level constant and observing the resultant accumulated change in core reactivity corresponding to successive rod motion steps.

f) Dynamic Xenon Transient Worth Measurements - Integral xenon worth transient measurements are to be made at elevated power, after a change in power level, by adjusting control rod position to maintain moderator average temperature and power level constant during the reactivity transient associated with the transient change in effective xenon concentration and observing the resultant accumulated change in core reactivity corresponding to successive compensating rod motion steps.

g) Depletion of Core Reactivity Due to Fue1 Burnup - Reactivity depletion rate measurements are to be made at operating power levels by observing the moderator average temperature compensation for burnup effects during an extended period of operation under closely limited conditions and relating the observed temperature compensation for burnup to reactivity by way of the differential moderator temperature coefficient. 


\subsubsection{PHASE III - POWER TESTING (Continued) \\ Elevated Power Transient Response Evaluation}

As the power level is increased during the initial power escalation, a series of transient response measurements will be made to determine plant response to load changes. The test technique in each case will consist of establishing the transient change in plant conditions and closely monitoring system response during and after the transient period.

\section{Elevated Power Determination of Power Distribution}

At successive power levels and in prescribed control rod configurations, measurements of flux and power distributions within the core will be made and nuclear hot channel factors will be evaluated. Use will be made of the Aero-Ball Flux System, and of the in-core temperature sensors, to determine the nuclear power and thermal and hydraulic conditions within the core. 


\section{SECTION \& \\ RESEARCH AND DEVELOPMENT}

\subsection{RESEARCH AND DEVELOPMENT}

\subsubsection{GENERAL}

Westinghouse Electric Corporation, under contract to the AEC (Contract No. AT(30-1) -3269), conducted a research and development program for specific efforts necessary to the design, construction and operation of the San Onofre Nuclear Generating Station. A substantial effort was expended to study the significant areas described below to verify the soundness of the analytical methods employed in the reactor core design, and to provide a. high degree of assurance that the design objectives will be met. The results of this program are documented in various quarterly progress and topical reports. Information obtained from this research and development program will be utilized for the development of future nuclear power plants in addition to San Onofre.

\subsubsection{DEVELOPMENT OF COMPUTATIONAL TECHNIQUES}

The research and development program developed an accurate, computerized method for the calculation of criticality at ambient and elevated temperatures. The program relies on auxiliary calculations based on Monte Carlo and integral transport theories to specify reactor lattice cell reaction rates. Using this program, it was possible to predict critical configurations and circumstances. These data, together with information obtained from other studies, have enabled the specification of requirements for San Onofre core fuel enrichments.

Fuel management studies were accomplished to develop the proper relation between fuel assembly core location and control rod programming. This made it possible to predict satisfactory power distributions commensurate with thermal and hydraulic design requirements. The studies defined refueling procedures and modes of operation for the multi-region San Onofre core, and improved the understanding of core phenomena.

Computer analyses also demonstrated the stability and resistance of the reactor core to xenon oscillations. It was found that the inherent damping afforded by the power coefficient of reactivity permitted a straightforward means of core operation while obtaining the desired core power.

Other studies have demonstrated the effects of chemical shim on the moderator coefficient of reactivity, as well as the consequences of a positive moderator coefficient. 


\subsubsection{PERFORMANCE OF CRITICAL EXPERIMENTS}

Concurrent with the development of the analytical techniques, a series of critical experiments was conducted to provide corroborating information where practicable. For example, measurements of critical configurations were made, where fuel loading was balanced against increasing concentrations of boron in the moderator. These measurements permitted evaluation of the computational techniques in determining thermal utilization in the presence of high concentrations of moderator poison, as well as leakage over a wide range.

\section{Rod Cluster Control}

Measurements of the effectiveness of the rod cluster control utilized in the San Onofre core were made. An analytical representation was developed which accurately determined control element worth. The representation, which is compatible with other limitations inherent in the techniques employed in core design, provides an accurate means of describing control and behavior under conditions of core operation.

Measurements of neutron flux distributions throughout core regions simulated actual heterogeneities (so called "fine structure" measurements) found in the operating core. These included the location of thicker clad fuel rods at positions of local power peaks. The measurements established the effectiveness of analysis in representing small lattice perturbations.

In addition, measurements were made of pertinent nuclear parameters and flux distributions in cores comprised of zirconium-clad, and both stainless stee 1 and zirconium-clad fuel rods. This latter information was necessary to simulate the expected transition from stainless steel to zirconium-clad fue 1 .

\subsubsection{DEVELOPMENT OF NEW FUEL ASSEMBLY DESIGN}

The San Onofre reactor core employs an advanced fuel assembly that embodies a number of important new features, such as the reduction of parasitic structural material in the active portion of the core. This reduction results in minimum fuel enrichment requirements during the lifetime of the core, enhancing plant operating economy.

An extensive test program was carried out to insure the satisfactory performance of this advanced fuel assembly. Two prototype test fuel assemblies of the rod cluster control canless design were fabricated, along with associated hardware.

The first test assembly, a hydraulic fuel assenbly together with a rod cluster control guide tube and control rod, was tested in the Westinghouse Reactor Evaluation Channel. In this channel, complete simulated reactor primary coolant chemistry was maintained. This included chlorides, oxygen, hydrogen, boric acid, etc., with coolant temperature at $575 \mathrm{~F}$ and a pressure of 1800 psig. Primary coolant flow conditions equivalent to 100 percent of the rated flow for San Onofre were maintained. Additional tests at 


\subsubsection{DEVELOPMENT OF NEW FUEL ASSEMBLY DESIGN (Continued)}

125 percent of rated flow were also conducted to establish the hydraulic lift of the fuel assembly. Pitot and static taps were located within the fuel assembly for verification of flow rates.

Total operating time to date for the rod cluster control canless assembly has been 1,245 hours, with 974 hours at temperatures of $550 \mathrm{~F}$ or better. The rod cluster control rod was cycled for a distance of 12,518 feet of linear travel, along with 514 recorded trips. Total travel was 16,630 feet. No mechanical failures of any type were encountered. Rod cluster wear and sheath wear were negligible. Close examination was made of 62 fuel rods, some partially and some fully removed from the fuel assembly. Absolutely no fretting or wear of any kind to the fuel rod or holding springs was indicated. The fuel assembly and control rod cluster has been reassembled and placed in the reactor channel, and will ultimately receive several thousand more hours of testing at simulated plant conditions.

Prior to the canless test, two tests of similar canned assemblies were conducted. These tests amassed an additiona1 832 trips and 19,947 linear feet of rod travel without any malfunction.

A second canless fuel assembly was built for mechanical evaluation in conjunction with simulated reactor fuel loading tests. The following tests were conducted:
a. Axial load-deflection characteristics
b. Lateral bending and deflection characteristics, including effective flexural rigidity
c. Torsional characteristics, including effective torsional rigidity

d. Natural frequency, including damping characteristics

e. Column stability

Instrumentation of the assembly consisted of strain gages to sense longitudinal, hoop and 45-degree strains in two axial planes 90 degrees apart; and dial gages to measure lateral and axial deflections. Loads were applied hydraulically and checked with Baldwin type load cells. Additional instrumentation consisted of a linear variable differential transformer, and associated instrumentation for natural frequency checks.

The assembly was also used for reactor loading checks. A test channel simulating four adjacent fuel assemblies was located in a deep pit. The fuel assembly was supported from an overhead crane, misaligned up to $3 / 8$ inch, lowered, and removed. Loading of the fuel element was recorded with a load cell placed between the fuel assembly and crane hook. No difficulties in loading or removing were encountered, nor was any damage sustained. 


\subsubsection{DEVELOPMENT OF CHEMICAL SHIM TECHNIQUUS}

A broad based study established the acceptability of the use of boric acid solutions, with or without $10^{-4}$ molar base ( $\mathrm{KOH}$ or LiOH), for chemical shim control of pressurized water reactors. The areas investigated because of possible problems through the use of these solutions were corrosion and wear of materials; the thermal and radiolytic stability of these solutions; and mechanisms, and the extent of undesirable reactivity effects due to chemical shim (boron hideout). Also studied was the basic physical chemistry of boric acid solutions, and the ability of boric acid and potassium borate to form ion exchange resins to purify the primary coolant. As a result of these studies, the acceptability of chemical shim control has been established.

A wide variety of materials were corrosion tested in an out-of-pile loop, in an in-pile loop, and at the Saxton Reactor for periods up to one year. Compared to water, boric acid chemical shim solutions have been found to differ only insignificantly with respect to corrosion effects for the 300 and 400 series stainless steels, Inconel 600 and related alloys, Zircaloy, and a variety of other alloys generally used in pressurized water reactor systems.

Tests of six different material combinations normally used in reactor applications showed that chemical shim had practically no effect, compared to water, on the wear properties of these combinations.

In autoclave, loop, and reactor tests of boric acid solutions with added $\mathrm{KOH}$ or $\mathrm{LiOH}$, in the quantities normally used in chemical shim solutions, no evidence of thermal or radiolytic instability was found. No loss of boron or base from the solutions was observed, nor was evidence of radiolytic decomposition of the water found in an in-pile loop test or in the Saxton Reactor.

It was recognized that the possibility of frecipitation, absorption, or other concentration of the neutron absorber boron at the fuel rod surfaces constituted a potential hazard in chemical shim plants. After extensive studies, investigators were able to delineate the conditions under which these processes might occur to a significant extent, and to establish that such conditions would not occur in a chemical shim plant. This conclusion was reached by theoretical analysis of experimental evidence, as well as close observation of the Saxton Reactor during more than a year's operation with chemical shim, both during normal and artificially induced high levels of corrosion product in the plant.

An investigation was conducted to determine the volatility, electric conductivity, and vapor pressure of boric acid solutions, with and without added base, as a function of temperature. These studies increased understanding of the ionization properties of boric acid, which in turn led to a satisfactory explanation of the effect of small additions of base ( $\mathrm{LiOH}$ or $\mathrm{KOH}$ ) to the high temperature $\mathrm{pH}$ of such solutions. These results were of value in interpreting observed effects of the addition of base to the primary coolant on the nuclear reactivity of both the Yankee and Saxton Reactors, with and without chemical shim control. 


\subsubsection{DEVELOPMENT OF CHEMICAL SHIM TECHNIQUES (Continued)}

It was shown experimentally in the laboratory, as well as during operation of the Saxton Reactor, that conventional ion exchange resins can be compatible with chemical shim solutions, yielding satisfactory decontamination factors and capacities for primary system purification. A theoretical interpretation of the results was also obtained.

\subsubsection{DEVELOPMENT OF PLANT CONTROL SYSTEMS}

An extensive reactor control and protection investigation was carried out to determine the requirements for a large chemical shim nuclear plant. The requirements for reactor control are more stringent in such a plant because of the reduced value of the moderator coefficient of reactivity due to large amounts of boric acid present in the reactor coolant system. For a short time at the beginning of core life, the coefficient is slightly positive.

Preferred compensation signals were determined for the control signals to improve control system stability and transient performance. These included signals for pressurizer pressure and the derivative of neutron flux.

A variable average coolant temperature program is employed. The overall system requirements, using the variable $\mathrm{T}_{\text {avg }}$ concept, include programmed pressurizer level and controlled steam dump on the turbine trip.

The plant protection system utilizes a calculated low pressure trip, which varies as a function of actual plant conditions in order to increase plant capability .

Improved plant simulation models were developed, particularly in the secondary system. Emphasis was placed on the transient behavior of large, vertical, U-tube steam generators. As a result of this effort, additional compensation signals were added to the normal three-element feedwater control system to improve response.

A steam dump control system was developed to handle large step load reductions without reactor trip. An automatic power cutback was devised to enable the plant to undergo a step reduction in electrical network frequency without reactor trip. 


\section{SECTION 9 \\ REACTIVITY FAULT ANALYSES}

\subsection{STARTUP}

\section{1 .1 GENERAL}

A startup incident or an uncontrolled rod withdrawal from a subcritical condition is extremely unlikely because of the protection provided. A continuous uncontrolled addition of positive reactivity during startup would result in an excessive rate of flux increase and a power excursion. Strict administrative controls will be followed at all times, and safety circuits, operated from nuclear instrumentation signals (reference Paragraph 6.1), are provided to safely terminate any such transient without core damage. Analysis of the kinetic behavior of the reactor and of the effectiveness of the safety devices shows that reactivity faults during startup are highly improbable and accidental core damage is effectively prevented.

The startup transient can proceed to a point requiring automatic reactor trip only if the control rods are continuously withdrawn or if the Chemical and Volume Control system malfunctions, and alarms are ignored. The range of reactivity insertion rates considered in the analysis of this transient includes the most severe conditions possible at startup.

While protection of the reactor core is the function of nuclear instrumentation in this case, pressure and level channels which respond to the power input to the reactor coolant would indefendently bring about a reactor trip in time to prevent an overpressure condition in the Reactor Coolant System, even if the protection from nuclear instrumentation were not considered. Multiple channels are employed in each means of protection, so that failure of any component will not prevent the desired signal from being given.

\subsubsection{FACILITY DESIGN ASPECTS}

If a continuous control rod withdrawal were initiated, and the startup rate alarms and indications initiated from the source and intermediate range channels were not considered, the transjent would be terminated by the following automatic safeguards:

a) Automatic Rod-stop Circuit - actuated by high startup rate signals from the source and intermediate range nuclear instrumentation channels, will prevent further rod withdrawal. The startup rate is measured by two independent channels in the source range and two channels in the intermediate range. A high startup rate signal from any one of the appropriate channels is sufficient to cause a rod stop.

b) Automatic Reactor Trip - actuated by a high startup rate signal from the intermediate range nuclear instrumentation provides further backup. This signal, which functions as a backup to the rod stop, will also be given if either one of two independent channels senses a high startup rate. 


\subsubsection{FACILITY DESIGN ASPECTS (Continued)}

c) Automatic Power Level Reactor Trip - set for a fraction of full power is also provided. This trip will be effected if any two of four independent power range channels give a high power signal in coincidence.

Because of their reliable design, failure of all of these protective circuits is inconceivable.

The nuclear power response to a continuous reactivity insertion is characterized by a very fast rise terminated by the reactivity feedback effect of the negative fuel temperature coefficient. This self-limitation of the initial power burst results from a sizeable prompt negative fuel temperature coefficient (Doppler effect) and is of prime importance during a startup accident since it limits the power to a tolerable level prior to external control action. After the initial power burst, the nuclear power is momentarily reduced and then if the accident is not terminated by a reactor trip, the nuclear power increases again, but at a much slower rate.

Termination of the startup transient by the high startup rate rod stop, high startup rate reactor trip, or the power range reactor trip, will prevent core damage. Other reactor protection channels, the variable low pressure trip, high pressure trip, and high pressurizer level trip (each actuated by coincidence of signals from any two of three independent channels), would terminate the startup transient independently before an overpressure condition could occur.

\section{Assumptions and Method of Analysis}

During a startup transient, the nuclear flux increases by many decades in a very short time interval. Analysis of this type of transient is by digital computation incorporating the neutron kinetics, including six delayed groups, and the core thermal and hydraulic equations. In addition to the nuclear flux response, the average fuel, clad and water temperatures, and also the hot spot heat flux response, are computed. Where applicable, the hot spot heat flux is used to calculate DNB ratios on the basis of the worst core power distribution expected during core life.

In the startup transient analysis, two distinct cases are studied, hot startup and cold startup. In the hot startup accident, the reactor is assumed to be initially at the normal operating pressure and the programmed temperatures for zero power. In the cold startup accident the temperature of the reactor is assumed to be 70 degrees Fahrenheit, and that pressure is assumed which results in the minimum terminal value of the DNB ratio. In either case the initial value of reactivity $\left(k_{0}=0.99\right)$ is selected from the range of expected values so that the greatest energy release will occur before any protective measures are taken.

In order to give conservative results for a startup transient, the following additional assumptions are made concerning the initial reactor conditions: 


\subsubsection{FACILITY DESIGN ASPECTS (Continued)}

a) Doppler Coefficient - since the magnitude of the nuclear power peak reached during the first part of the transient, for any given rate of reactivity insertion, is strongly dependent on the fuel temperature reactivity coefficient, the least negative design value is used for the startup accident $\left(\alpha_{\mathrm{f}}=-1 \times 10^{-5} \delta \mathrm{k} / \mathrm{F}\right)$.

b) Moderator Coefficient - the contribution of the moderator reactivity coefficient is negligible during the initial part of the transient because the heat transfer time constant between the fuel and the moderator is much longer than the nuclear flux response time constant. However, after the initial nuclear flux peak, the succteding rate of power increase is affected by the moderator reactivity coefficient. Accordingly, the most positive expected value is used for the moderator coefficient, since this yields the maximum rate of power increase $\left(\alpha_{\mathrm{w}}=+1 \times 10^{-4} \delta \mathrm{k} / \mathrm{F}\right)$.

c) Reactivity Insertion Rate - for a given set of reactivity coefficients, the maximum energy release in the fuel. corresponds to the maximum rate of reactivity insertion; thus, the reactivity insertion rate corresponding to withdrawal of the control rod group at the maximum velocity in the region of maximum reactivity worth is assumed in the analysis. This may be either a shutdown group or a control group, with the amount of reactivity inserted being limited by the maximum rod drive speed and the worth of the rod group. Reactivity insertion by rod withdrawal $\left(\delta \mathrm{k} / \mathrm{sec}=2 \times 10^{-4}\right)$ is inherently faster than that which can be produced by dilution of boron with the Chemical and Volume Control system. The significant aspects of reactivity addition by dilution of boron are covered in Paragraph 9.3, Boron Dilution.

d) Reactor Trip - the behavior of the reactor is analyzed as though no protective trip were actuated until the overpower reactor trip set point is reached. The most adverse combination of instrument and set point errors, as well as delays for trip signal. actuation and rod release are taken into account. Also, the rate of negative reactivity insertion corresponding to the trip action is computed based on the assumption that the highest worth rod is stuck in its fully withdrawn position.

\section{Results}

\section{Cold Startup}

The results of an uncontrolled control rod withdrawal with a constant insertion rate of $2 \times 10^{-4} \delta \mathrm{k}$ per second from a cold coolant condition of 70 degrees Fahrenheit are shown in Figures 9.1 through 9.3. This reactivity insertion is the maximum rate for maximum control rod speed of 15 inches per minute and a total group worth of approximately 3.25 percent. This is a conservative assumption since no single programmed group of rod cluster control assemblies of this magnitude exists in the core. Also, no credit is taken in the transient for the fact that this maximum incremental worth would exist over only a small portion off core travel. The maximum reactivity insertion rate achievab $\frac{1}{5}$ with dilution by the Chemical and Volume Control System is $1.0 \times 10^{-5} \delta \mathrm{k}$ per second (reference Paragraph 9.3, Boron Dilution), which is a relatively insignificant rate compared to the $2.0 \times 10^{-4} \delta \mathrm{k}$ per second. 


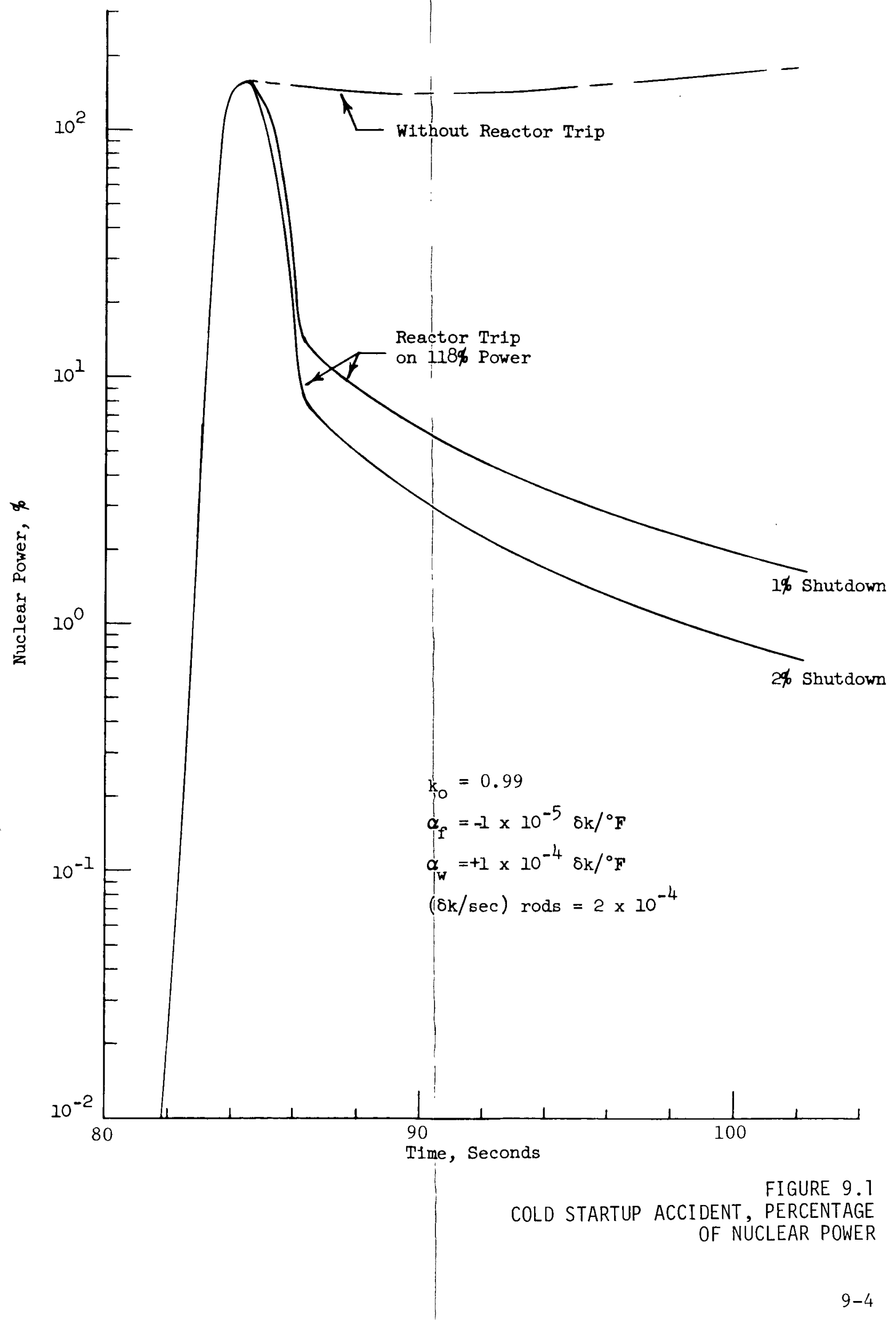




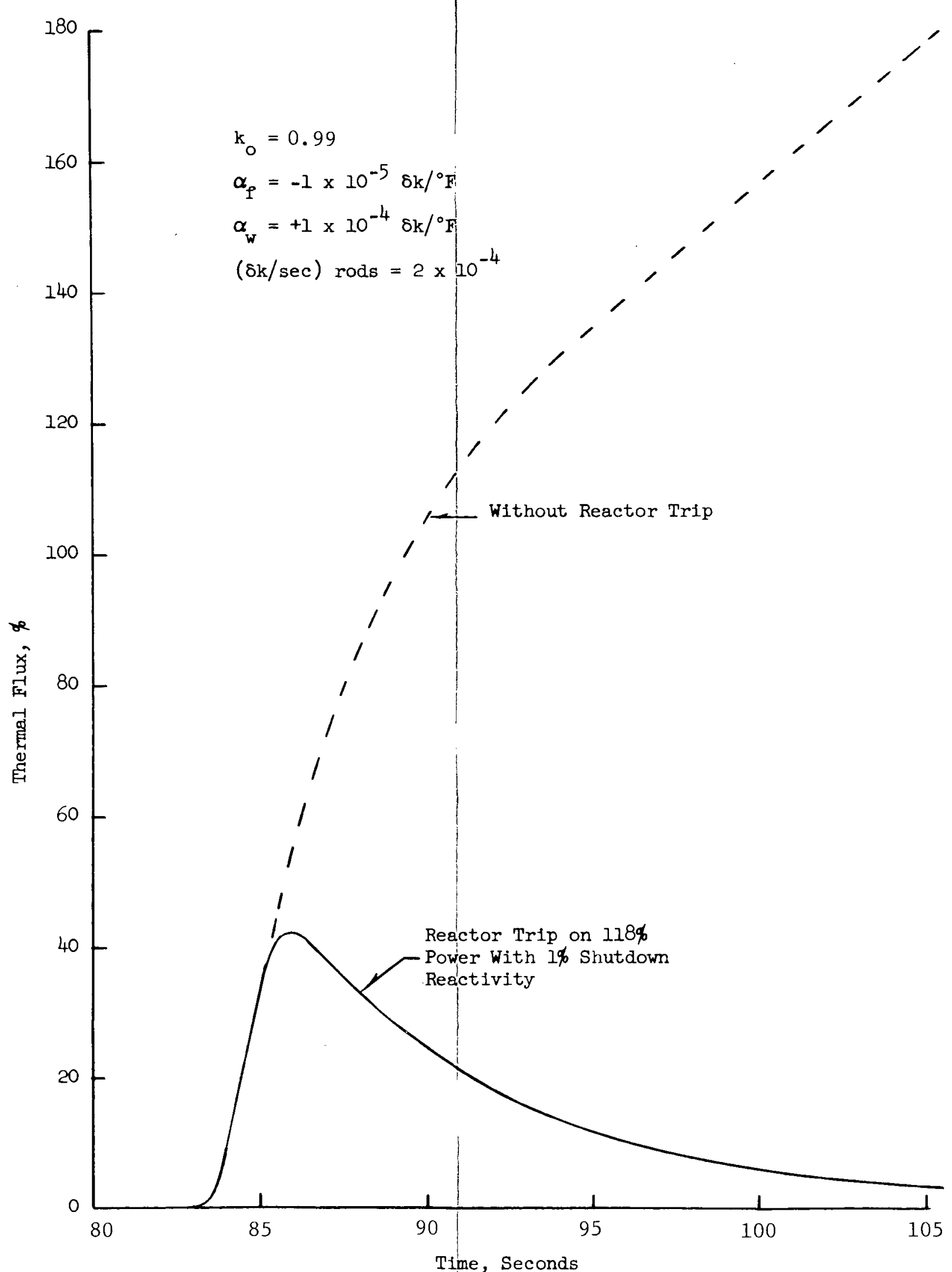

FIGURE 9.2

COLD STARTUP ACCIDENT, PERCENTAGE

OF THERMAL FLUX 


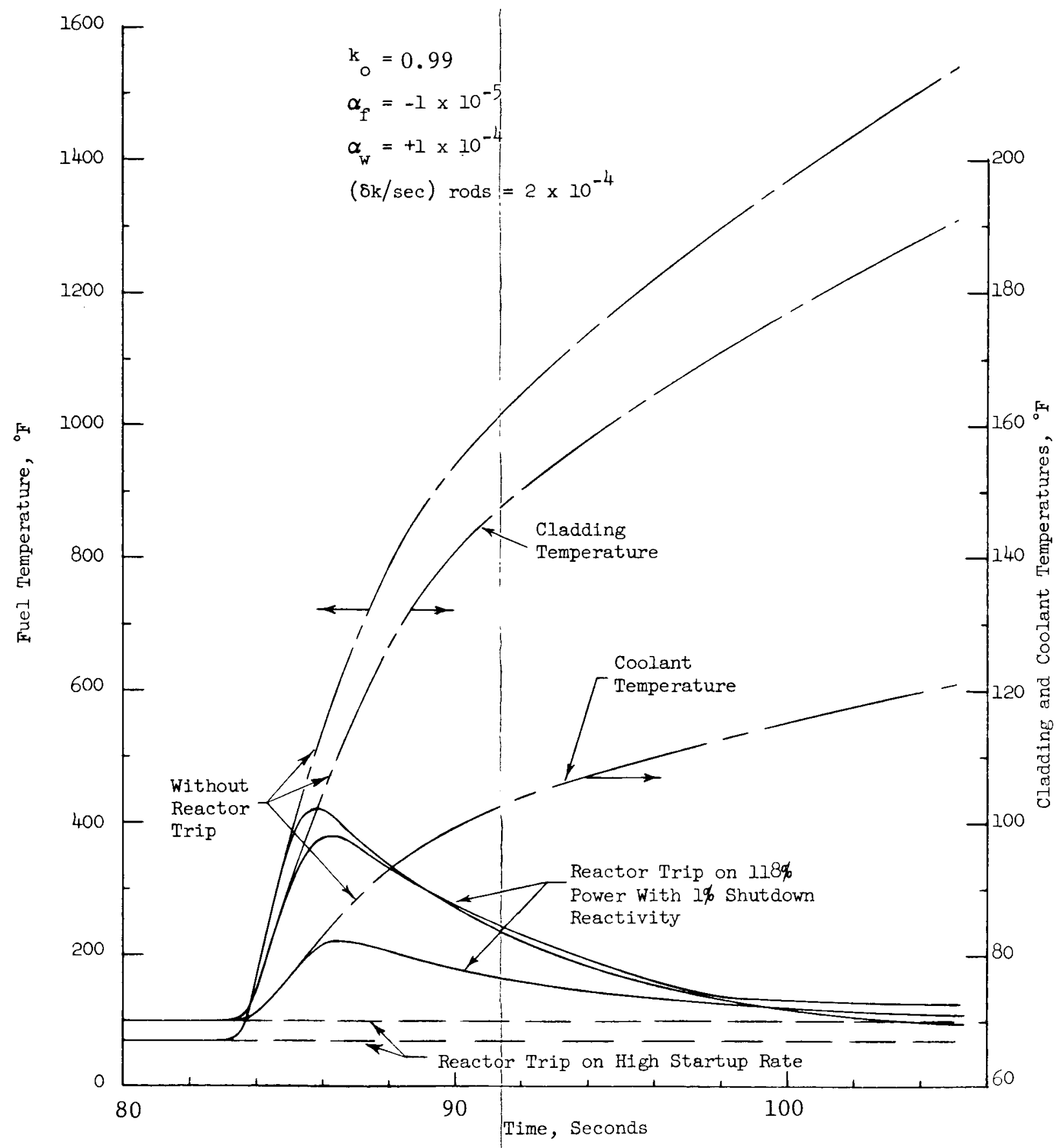

FIGURE 9.3

COLD STARTUP ACCIDENT, FUEL
TEMPERATURE 


\subsubsection{FACILITY DESIGN ASPECTS (Continued)}

The reactor is assumed to be subcritical $\left(k_{\text {eff }}=0.99\right)$. The Doppler coefficient is assumed to be equal to the conservative value of $-1.0 \times 10^{-5} \delta \mathrm{k}$ per degree Fahrenheit. (Reference paragraph 3.4 ) That section indicates a Doppler coefficient in excess of $-2.0 \times 10^{-5} \varepsilon \mathrm{k}$ per degree Fahrenheit at low effective temperatures. The reactivity feedback from Doppler provides the primary power cutback as shown in Figure 9.1 where there is a sharp break in the nuclear power transient at approximately 84 seconds. The effect of a reactor trip is also shown in this figure where the trip is conservatively assumed to occur at 118 percent power. The low power (25 percent) reactor trip will be in effect during subpower operation. It can be seen that the low power (25 percent) trip would occur at approximately 83.4 seconds while the 118 percent trip occurs at 83.8 seconds. The control rod insertion on reactor trip was assumed to follow the insertion versus time function described in Appendix $A$. The nuclear power decay is shown with two assumed values of shutdown reactivity and there is little significant difference except in the 0 to 10 percent power range. This shutdown is in addition to the reactivity inserted by the rods which are assumed to accidentally withdraw. The thermal flux response, of interest for DNB considerations, is shown in Figure 9.2. The beneficial effect of the inherent thermal $1 \mathrm{ag}$ in the fuel is evidenced by the peak flux equal to 42 percent of the ful1-load value reached during the transient. The heat transfer coefficient assumed from the fuel to coolant was a conservative high value, equivalent to assuming air in the gap between pellet and cladding rather than fission gases, thus yielding the most rapid heat transfer to the coolant. The fuel and cladding temperatures are shown in Figure 9.3. Also shown in this figure is the maximum coolant average temperature reached of 82 degrees Fahrenheit and thus there is no significant reactivity addition due to the positive moderator coefficient of reactivity. The positive moderator coefficient assumed for this case was $\alpha_{w}=+1.0 \mathrm{x}$ $10^{-4} \delta \mathrm{k}$ per degree Fahrenheit, which is quite large compared to the predicted values in Paragraph 3.4, Reactor Nuclear Design. The temperatures will not increase if the high startup rate trip occurs at 64 seconds.

There is a large margin to DNB during the transient since the rod surface heat flux-remains low, and there is a high degree of subcooling at all times in the core.

\section{Hot Startup}

Figures 9.4 through 9.7 show the transient behavior for a startup accident from hot zero power conditions with average coolant temperature equal to 535 degrees Fahrenheit. Other plant conditions are the same as assumed for the cold startup case, i.e., $k_{\text {eff }}=0.99, \alpha_{f}=-1 \times 10^{-5} \delta \mathrm{k}$ per degree Fahrenheit and $\alpha_{w}=+1 \times 10^{-4} 8 \mathrm{k}$ per degree Fahrenheit. The rate of reactivity insertion is also the same, $2 \times 10^{-4} \delta \mathrm{k}$ per second.

The average thermal heat flux transient, assuming a reactor trip at 118 percent power, is shown in Figure 9.5. The peak average thermal heat flux is approximately the same as in cold startup case. The increase in average 


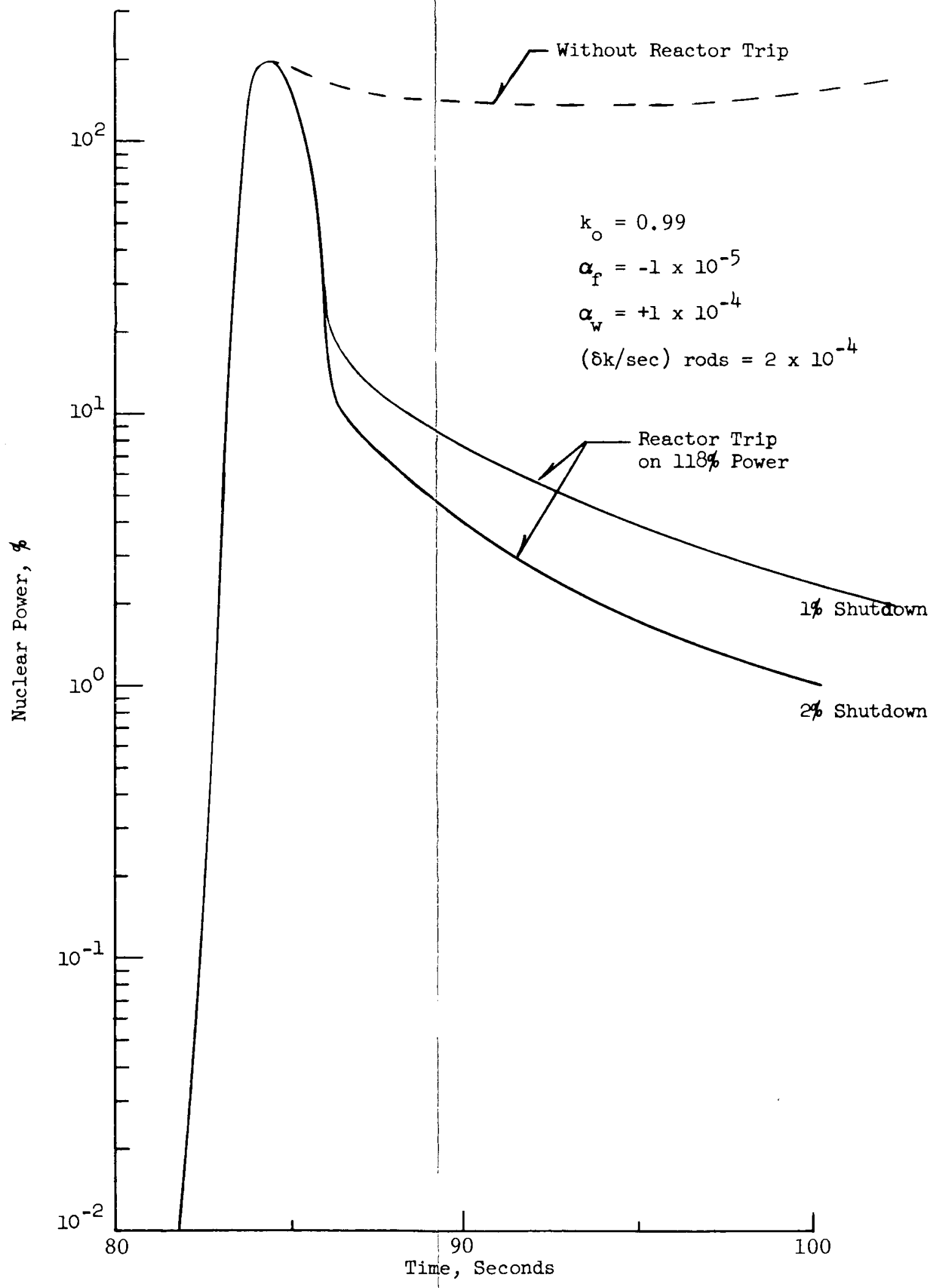

FIGURE 9.4 HOT STARTUP ACCIDENT, PERCENTAGE OF NUCLEAR POWER 


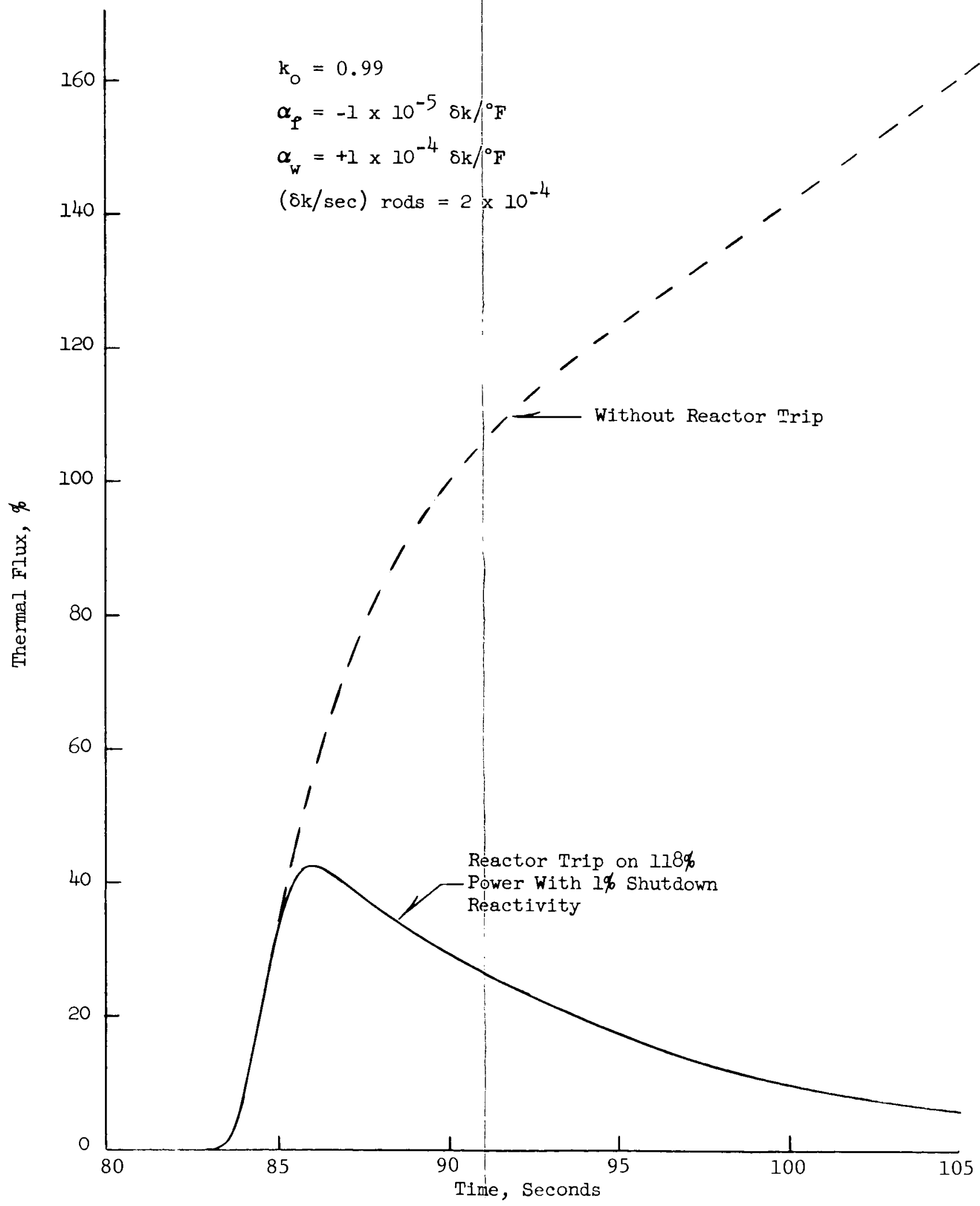

FIGURE 9.5

HOT STARTUP ACCIDENT, PERCENTAGE OF THERMAL FLUX 


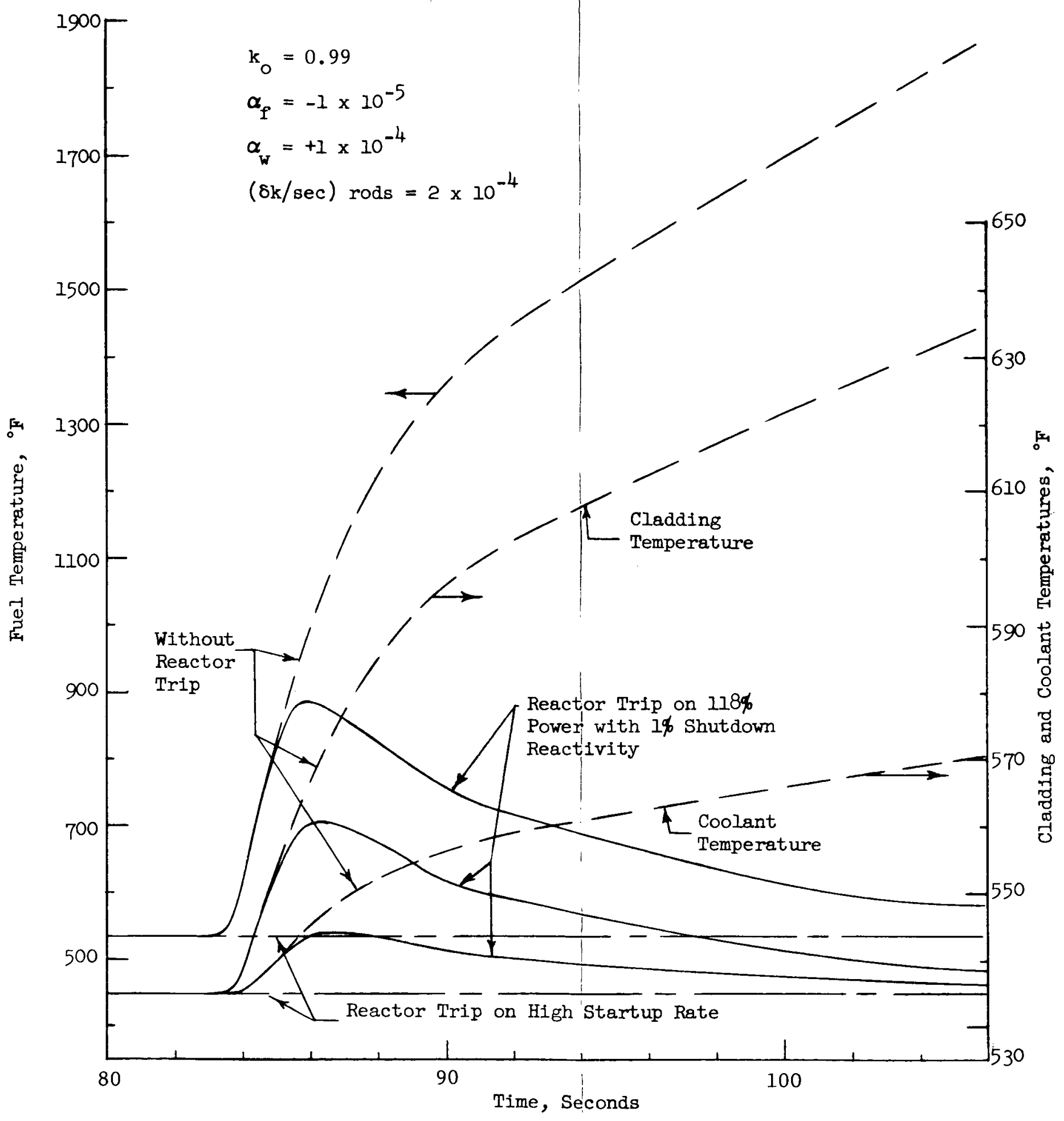

FIGURE 9.6

HOT STARTUP TEMPERATURE TRANSIENTS 


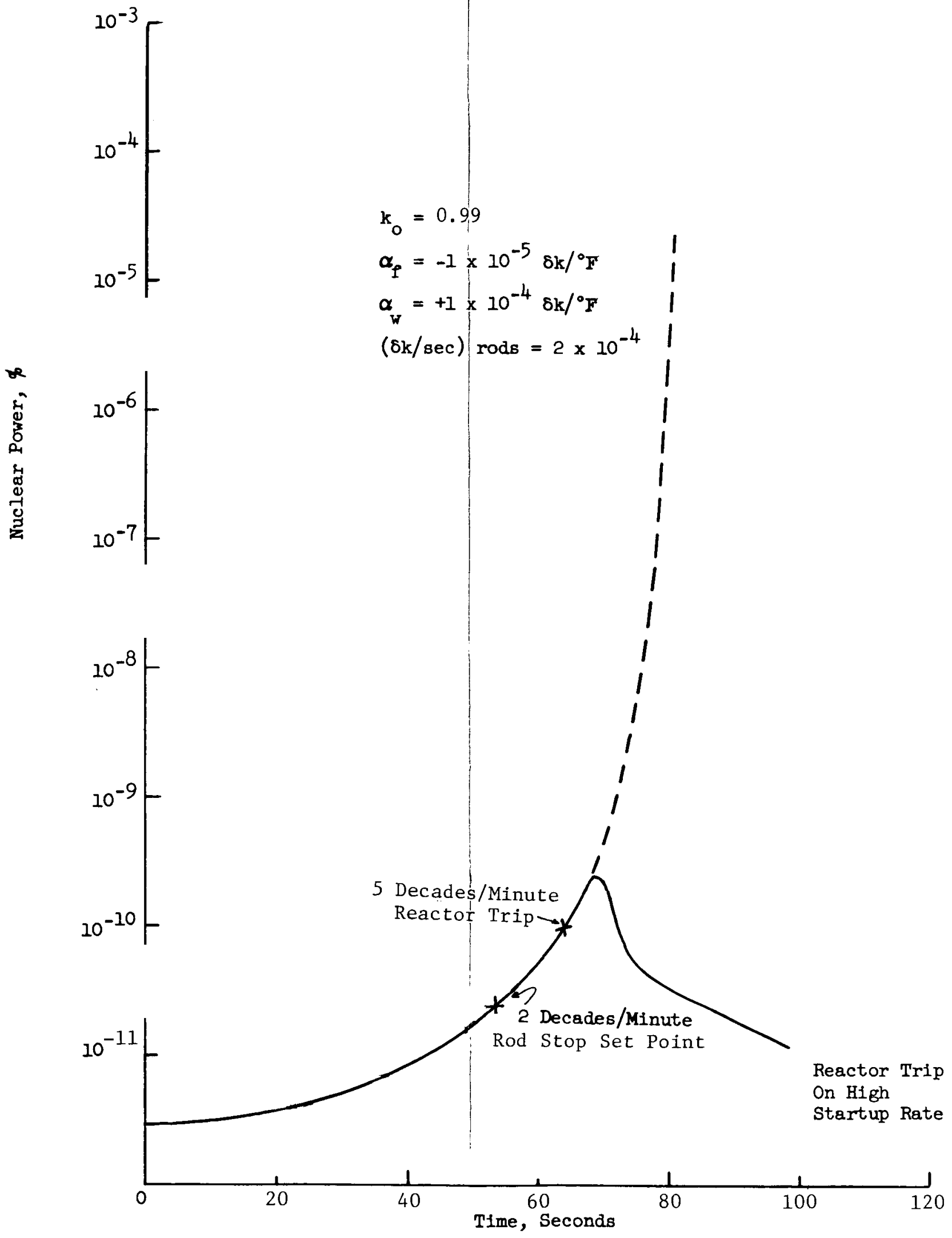

FIGURE 9.7 


\subsubsection{FACILITY DESIGN ASPECTS (Continued)}

coolant temperature, as shown in Figure 9.6, assuming a reactor trip at 118 percent power, is nine degrees Fahrenheit, and is again insignificant with respect to positive moderator reactivity feedback. All the temperatures wil1 remain at 535 degrees Fahrenheit if the high startup rate trip functions at 64 seconds as shown in Figure 9.7. The initia1 neutron source level is assumed to be 170 neutrons per cubic centimeter per second.

Since the peak average heat flux is only 42 percent of the steady state design value, the margin to DNB presents no problem during the accident. The $W-3$ DNB correlation is used to deternine the minimum DNB ratio for the hot startup accident (Paragraph 3.3, Reactor Thermal - Hydraulic Design). The minimum DNB ratio occurs at approximately 86 seconds and is equal to 4.6, which is considerably higher than the minimum DNB ratio of 1.3 required. Were the accident to proceed without a reactor trip, it is conservatively calculated that a minimum DNB ratio of 1.3 would not be reached until approximately 95 seconds.

A parameter study was performed to investigate the sensitivity of peak nuclear power reached to initial multiplication factor, moderator coefficient of reactivity $\left(\alpha_{\mathrm{W}}\right)$, Doppler coefficient of reactivity $\left(\alpha_{\mathrm{f}}\right)$ and the heat transfer coefficient from the fuel to clad (UA) $f$. The results are presented in Table 9.1. It can be seen that the Doppler coefficient provides the primary feedback mechanism to reduce the rapid nuclear power excursion. The effect of initial shutdown margin on the peak transients is insignificant. An initial multiplication factor of 0.99 is used for the accident.

Table 9.1 Hot Startup Accident

\begin{tabular}{|c|c|c|c|c|}
\hline $\begin{array}{c}\text { Initial } \\
\text { Multiplication } \\
\text { Factor }\end{array}$ & $\begin{array}{c}\alpha_{\mathrm{w}} \\
\left(10^{4} \delta \mathrm{k} / \mathrm{F}\right)\end{array}$ & $\begin{array}{c}\alpha_{\mathrm{f}} \\
\left(10^{5} \delta \mathrm{k} / \mathrm{F}\right)\end{array}$ & $\begin{array}{c}\text { (UA) } \\
\left(10^{-3} \text { Btu/sec F }\right)\end{array}$ & $\begin{array}{c}\text { Peak Nuclear } \\
\text { Power } \\
\text { Percent }\end{array}$ \\
\hline 1.0 & +1 & -1 & 1.65 & 186.5 \\
1.0 & +1 & -1 & 0.7385 & 174.2 \\
1.0 & +1 & -2 & 1.65 & 88.8 \\
1.0 & +0.5 & -1 & 1.65 & 174.9 \\
1.0 & -1 & -1 & 1.65 & 158.2 \\
0.995 & +1 & -1 & 1.65 & 199.0 \\
0.99 & +1 & -1 & 1.65 & 199.5 \\
\hline
\end{tabular}




\section{1 .3 CONCLUSIONS}

Although very conservative assumptions were used in this analysis, the results indicate that the reactor core is safely protected from both the cold startup and the hot startup accidents. The peak thermal heat flux is well below the steady state design value and the minimum DNB ratio is considerably higher than the minimum DNB ratio of 1.3 required. 


\subsection{ROD WITHDRAWAL AT POWER}

\subsubsection{GENERAL}

A continuous withdrawal of control rods at power without an increase in load in the turbine cycle is considered here. The response of the core is an increase in heat flux. Since the corresponding increase in heat extraction from the steam generator is a relatively small effect, there is a net increase in the reactor coolant temperature. Unless terminated by manual or automatic action, this power mismatch and resultant coolant temperature rise would eventually result in DNB. To prevent the possibility of damage to the cladding, therefore, the Reactor Control and Protection System (reference Paragraph 6.2) is designed to terminate any such transient with an adequate margin to $\mathrm{DNB}$.

The third barrier to fission product release, the Reactor Coolant System (reference Paragraph 3.5), is not subjected to excessive pressure in this transient, and protection is also provided by the Reactor Protection System. Reactor Protection System circuits independent of those provided for core protection would also terminate the transient before water level or pressure in the pressurizer could exceed safe limits. These include the high pressurizer pressure and level trip circuits.

A rod withdrawal transient could be initiated by a malfunction of the automatic Reactor Control and Protection Systern. In either case, both slow and fast reactivity additions, up to the maximum possible rate, must be considered because of the several ways (heat flux, temperature and pressure) in which the DNB condition can be approached.

\subsubsection{FACILITY DESIGN ASPECTS}

The automatic features of the Reactor Control and Protection System which prevent core damage in a rod withdrawal transient at power include the following:

a. Nuclear power range instrumentation actuates an automatic rod withdrawal stop signal if one out of four power range channels exceeds an overpower set point ( 10.5 percent).

b. Nuclear power range instrumentation actuates a reactor trip if any two out of four power range channels exceed an overpower set point (109 percent).

c. Reactor trip is actuated if any two out of three pressure channels indicate less than a variable low pressure set point. The low pressure trip set point is automatically adjusted for power and temperature to limit the amount of boiling in the core at the trip condition.

d. A high pressure reactor trip, actuated from any two out of three pressure channels, is set at a fixed point $(2,250$ psia). 


\subsubsection{FACILITY DESIGN ASPECTS (Continued)}

The manner in which the combination of overpower and variable low pressure trips provide protection over a full range of reactivity insertion rates is illustrated in Figure 9.8. This diagram represents the possible conditions of thermal power and coolant inlet temperatures in a two-dimensional plot. The boundaries of operation defined by the overpower trip and the variable low pressure trip are represented as "protection lines" on this diagram - the overpower trip as a vertical line at the maximum value of nuclear power and the variable low pressure trip as family of sloping lines whose temperature intercepts increase with increasing pressure. The protection lines are drawn to include all adverse instrumentation and set point errors, so that under nominal conditions trip would occur well within the area bounded by these lines. A comparison between nominal and worst case trip conditions for this type of transient is presented in Paragraph 9.2.4. A maximum steady state operating condition for the reactor is shown as point 0 on Figure 9.8 .

A transient caused by reactivity insertion would result in movement of the operating point generally in the direction of increased power and inlet temperature, i.e., to the right and upward on Figure 9.8. A rapid reactivity addition can cause power increase which brings the reactor to the overpower limit before the inlet temperature can respond, i.e., along path $0-A$. A very slow reactivity addition can cause a net increase in inlet temperature to the variable low pressure trip point with only a smaller rise in thermal power, i.e., along path $0-B$. Other transients, corresponding to reactivity addition at intermediate rates, would be represented by paths lying between $\mathrm{O}-\mathrm{A}$ and $\mathrm{O}-\mathrm{B}$.

The utility of the diagram just described is in the fact that the operating limit imposed by any given DNB ratio can be represented as a line on this coordinate system. In Figure 9.8, the line represents the locus of conditions for which DNBR equals 1.30 at 2,150 psie and 2,250 psia. All points below and to the left of this line have a DNB ratio greater than this value. The diagram shows that DNB is prevented for all cases if the area enclosed within the maximum protection lines is not traversed by the applicable DNB ratio line at any point.

The overpower trip representation on the diagram is invariant with pressure. The variable low pressure trip line at 2,150 psia lies below the 2,250 psia line, however, there being a smaller margin of subcooling at any operating temperature. Conversely, higher pressure raises the intercept of the variable low pressure trip 1ine. At pressures greater than 2,250 psia, the limiting line falls below the low pressure trip line. Duplicate spray and relief valves are provided at the pressurizer to reliably limit transient overpressure to 2,250 psia for normal operating transients. For a complete loss of load without an immediate trip, or for a rod withdrawal transient from low power levels, the power mismatch can result in a rate of volume insurge to the pressurizer greater than the capacity of the power operated relief valves to limit pressure to 2,250 psia. For these transients a fixed high pressure trip, detected by two out of three pressure channels, will terminate the transient and limit pcessure to 2,250 psia. There is also a fixed lower limit for the variable low pressure reactor trip set at 1,600 psia. 


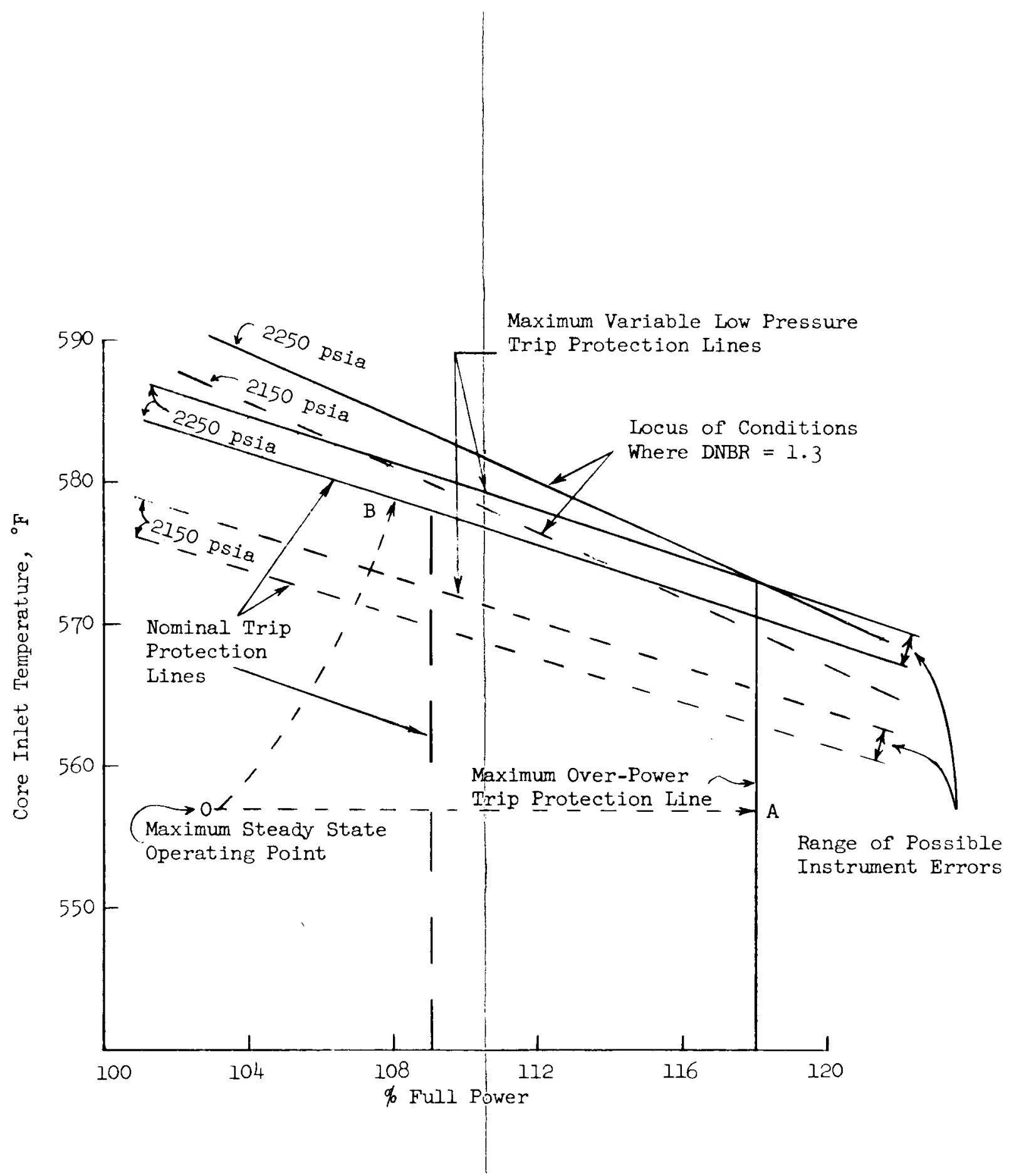

FIGURE 9.8 OVER-POWER, OVER-TEMPERATURE PROTECTION DIAGRAM 


\subsubsection{FACILITY DESIGN ASPECTS (Continued)}

From the arrangement of the protection Lines in Figure 9.8, it is apparent that only those rod withdrawal transients which result in power-temperature conditions near the intersection of the protection lines would even approach the minimum DNBR limit. Considering the reliability of the protective circuits and the fact that reactor operation is permitted only when these circuits are functioning, the possibility of core damage or overpressure from rod withdrawal at power is extremely remote.

The overpower rod stop is provided to reduce the probability of an overpower trip caused by overshoot when moving control rods. This is achieved by stopping the rods at a flux level short of: the trip set point.

The pressurizer high level trip, actuated by coincidence of two out of three high level signals, is a backup system provided to guard against water discharge from the pressurizer safety valves in the event of a sustained overpower condition. By preserving the required conditions for design safety valve capacity, the high level trip assures Reactor Coolant System protection independent of the means of core protection.

Assumptions and Method of Analysis

The control rod withdrawal accident analysis employs both analog and digital computer techniques. From the digital computer, the value of the variable low pressure trip set point is determined as a function of power, temperature and pressure such that trip is obtained over the range of interest before the DNB ratio becomes less than 1.30. This set point is then programmed, along with the overpower trip set point, with the full plant simulation on the analog computer.

Figures $9.9,9.10$, and 9.11 represent the results of a series of computations with varying input parameters. These curves are presented to show generally the effects of key parameters on the approach to DNB, and will clarify the basis for selection of pessimistic assumptions for the limiting case.

Figure 9.9 shows terminal DNB ratio as a function of reciprocal reactivity addition rate for various moderator coefficients in the range of importance. The limiting DNB ratio (i.e., the minimum approach to DNB) for each curve exists at a point of abrupt slope change corresponding to the intersection of the overpower and variable low pressure protection lines. The portion of the curves to the left of the abruptslope change corresponds to termination by the overpower trip, while the portion to the right corresponds to termination by the variable low pressure trip. The minimum DNB ratio for the limiting condition is essentially independent of moderator coefficient, although the reactivity addition rate for minimum DNB ratio becomes slower as the coefficient becomes more positive.

Figure 9.10 illustrates the effect of pressure on terminal DNB ratio in rod withdrawal transients. In the curve marked without spray the pressure rises to the relief valve setting of 2,250 psia in the slower transients. With spray, pressure is suppressed to a value nearer the nominal value of 2,100 psia, and consequently, the variable low pressure trip occurs at a lower 


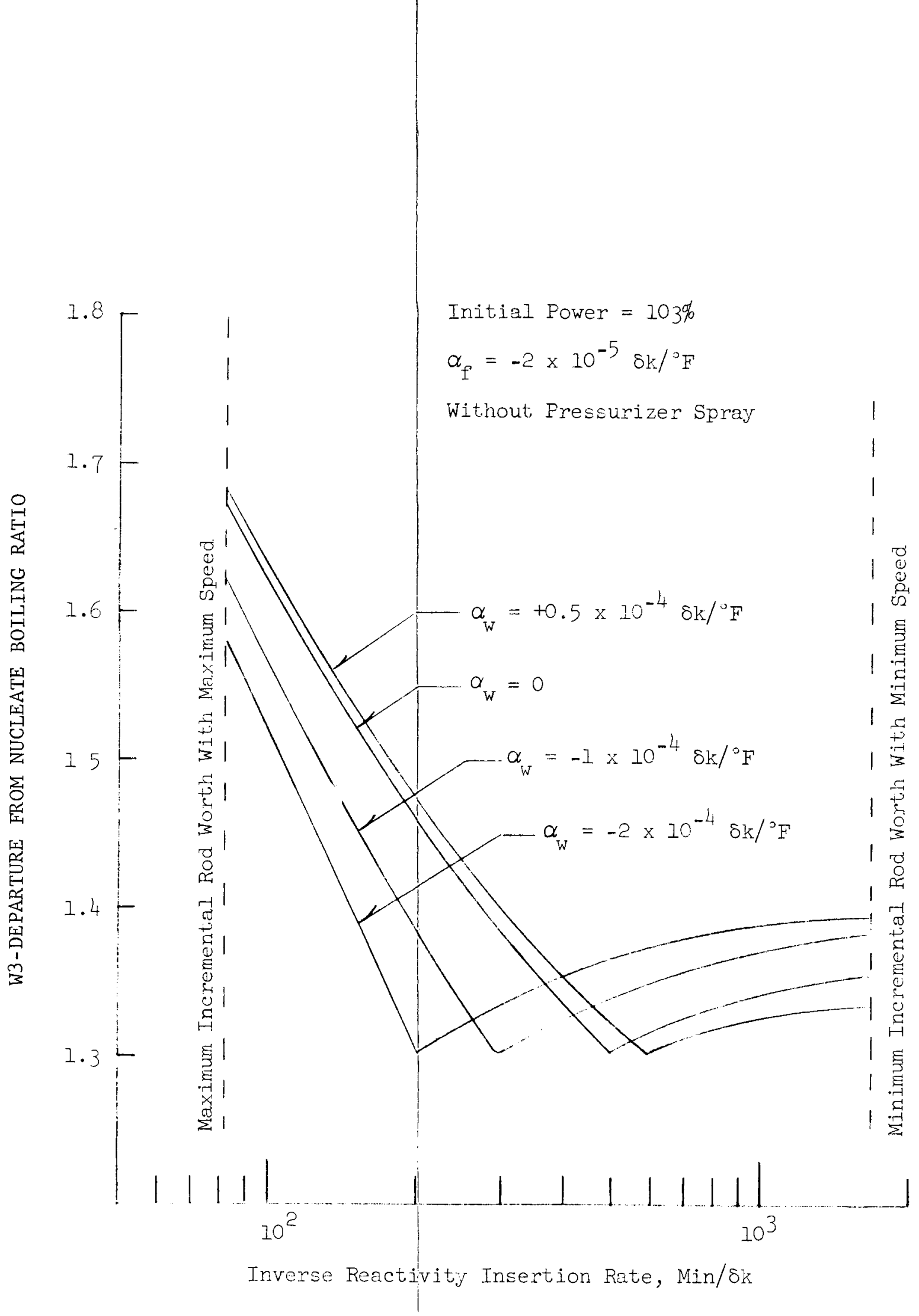

FIGURE 9.9 EFFECT OF MODERATOR COEFFICIENT ON TERMINAL W3 DNB RATIO IN ROD WI THDRAWAL TRANSIENTS 


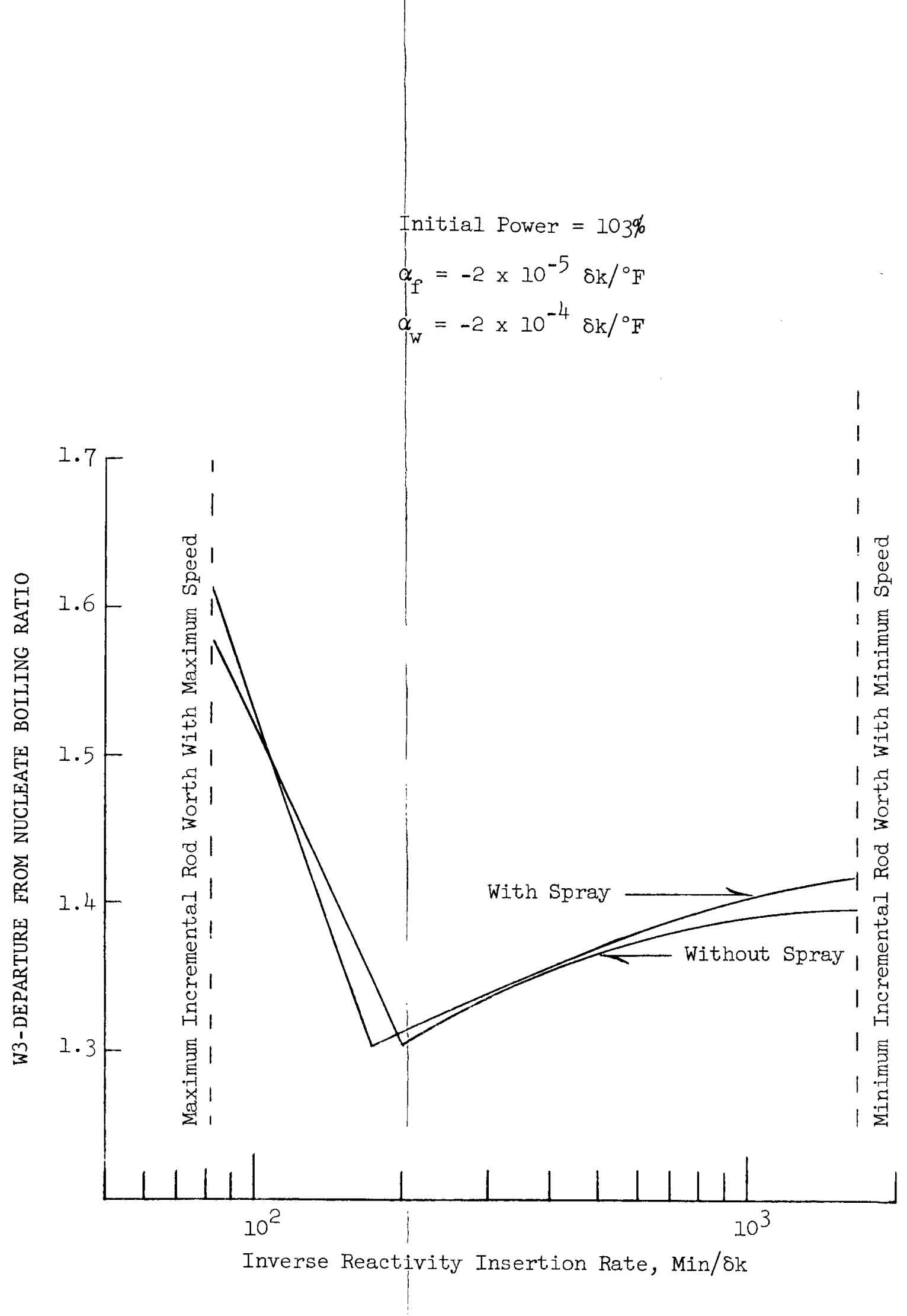

FIGURE 9.10 EFFECT OF PRESSURIZER SPRAY ON TERMINAL W3 DNB RATIO IN ROD WITHDRAWAL TRANSIENTS 


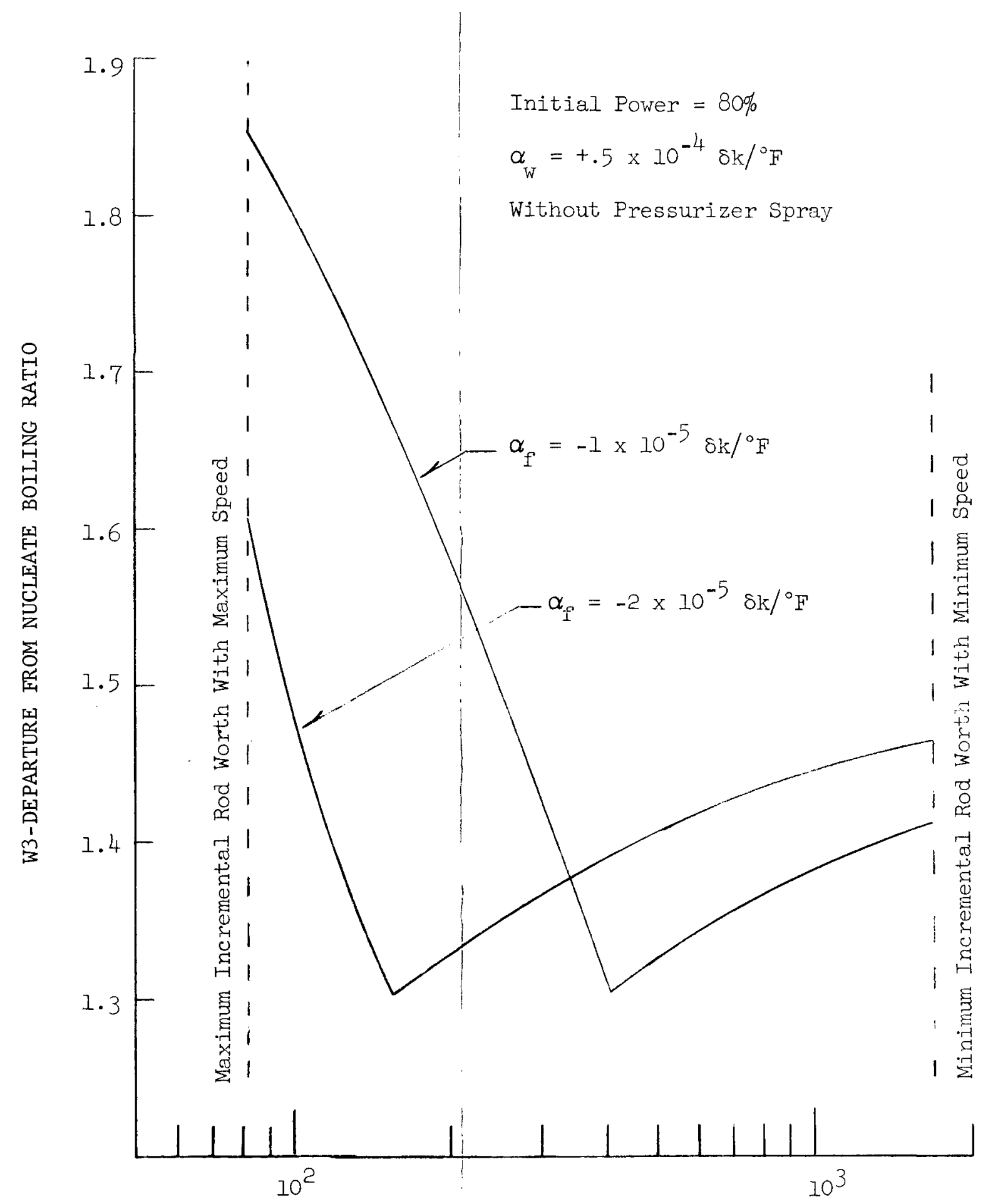

Inverse Reactivity Insertion Rate, Min/Sk

FIGURE 9.11 TERMINAL W3 DNB RATIO IN ROD WITHDRAWAL TRANSIENTS FROM REDUCED

POWER LEVEL 


\subsubsection{FACILITY DESIGN ASPECTS (Continued)}

temperature. This lower temperature does not significantly affect the DNB ratio at trip since the trip occurs at the same exit quality in the core. Note that the limiting DNB ratio is unaffected, indicating that this condition is approached over a relatively small range of addition rates which are too rapid for spray to be effective. For limiting cases, the spray is assumed to be ineffective and the pressure is taken at the relief valve limit.

Figure 9.11 shows, by comparison with Figure 9.9, the effect of initial power on terminal DNB ratio for the case of the most positive moderator coefficient $\left(\alpha_{\mathrm{W}}=+0.5 \times 10^{-4} \delta \mathrm{k} / \mathrm{F}\right)$ and for the same fuel coefficient $\left(\alpha_{f}=-2.0 \times 10^{-5} \delta_{\mathrm{k}} / \mathrm{F}\right)$. For the case where the transient starts from a partial power condition, it is seen that the limiting DNB ratio is essentially the same, that the variable low pressure trip is the governing protection over a wider range of addition rates, and that somewhat lower terminal DNB ratios are reached in those fast transients which are terminated by the overpower trip.

A typical result with a smaller fuel cemperature coefficient, $\alpha$, shown for comparison in Figure 9.11, indicates the limiting DNB ratio is not sensitive to changes in $\alpha f$ over the range $-1.0 \times 10^{-5}$ to $-2.0 \times 10^{-5} 8 \mathrm{k}$ per degree Fahrenheit. The essential effect of a less negative $\alpha_{f}$ is that the terminal DNB ratio resembles that of a slower reactivity addition rate.

For the case of an overpower trip, the time delay for breaker motion and rod release may allow an appreciable overshoot in nuclear power. This effect is counteracted by a much greater la.g, however, between the nuclear flux and thermal flux levels due to thermal inertia of the fuel. For a slow reactivity accident which could result in a variable low pressure trip, the thermal flux may be following the nuclear flux more closely, but with the slower rate the delay in initiation of the trip is not significant. Thus, in either case, the results are not affected by the rate of negative reactivity insertion following trip.

In summary, to give conservative results, the initial operating point is assumed for analysis to be the maximum power level, the maximum inlet temperature, and the maximum primary pressure consistent with steady state operation. This will give the minimum initial margin to DNB and, since the thermal flux lags the nuclear flux, the minimum margin at the point of reactor trip for any reactivity addition rate.

\section{$\underline{\text { Results }}$}

Three rod withdrawal transients are shown. Figure 9.12 shows the response of certain plant variables for the maximum reactivity insertion rate of $2 \times 10^{-4} \delta \mathrm{k}$ per sec. Spray is assumed to be working but the transient is terminated so rapidly there is little spray effect. The maximum positive moderator coefficient of $+0.5 \times 10^{-4} \delta \mathrm{k}$ per degree Fahrenheit was used and a minimum Doppler coefficient of $-1 \times 10^{-5} \delta \mathrm{k}$ per degree Fahrenheit was assumed. The initial power level is 103 percent of full power $(1.03 \times 1,347$ Mwt). The reactor trip occurs from high nuclear power (118 percent) in 


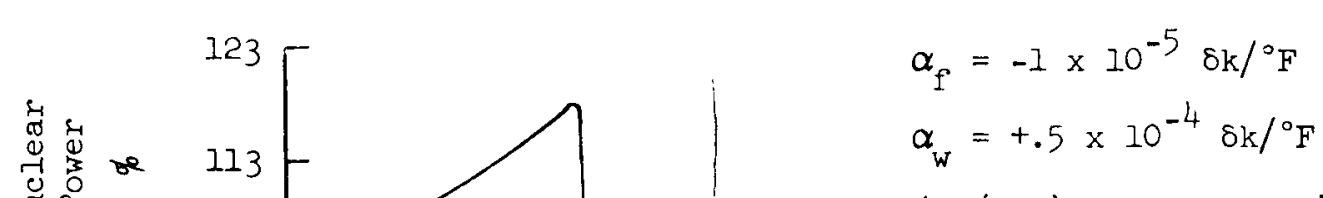

$\alpha_{f}=-I \times 10^{-5} \delta \mathrm{k} /{ }^{\circ} \mathrm{F}$

$\alpha_{\mathrm{w}}=+.5 \times 10^{-4} \mathrm{\delta k} /{ }^{\circ} \mathrm{F}$

$(\delta \mathrm{k} / \mathrm{sec}) \operatorname{rod}=2 \times 10^{-4}$
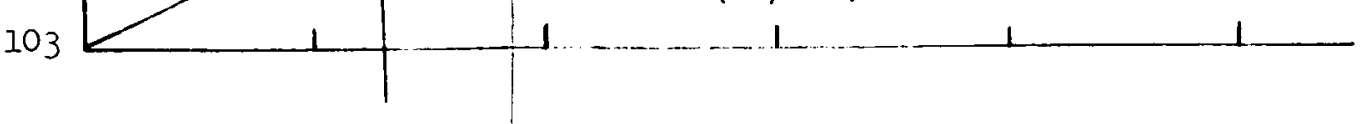

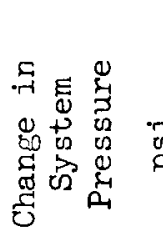

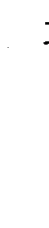

100

我

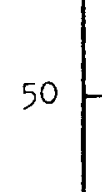

.
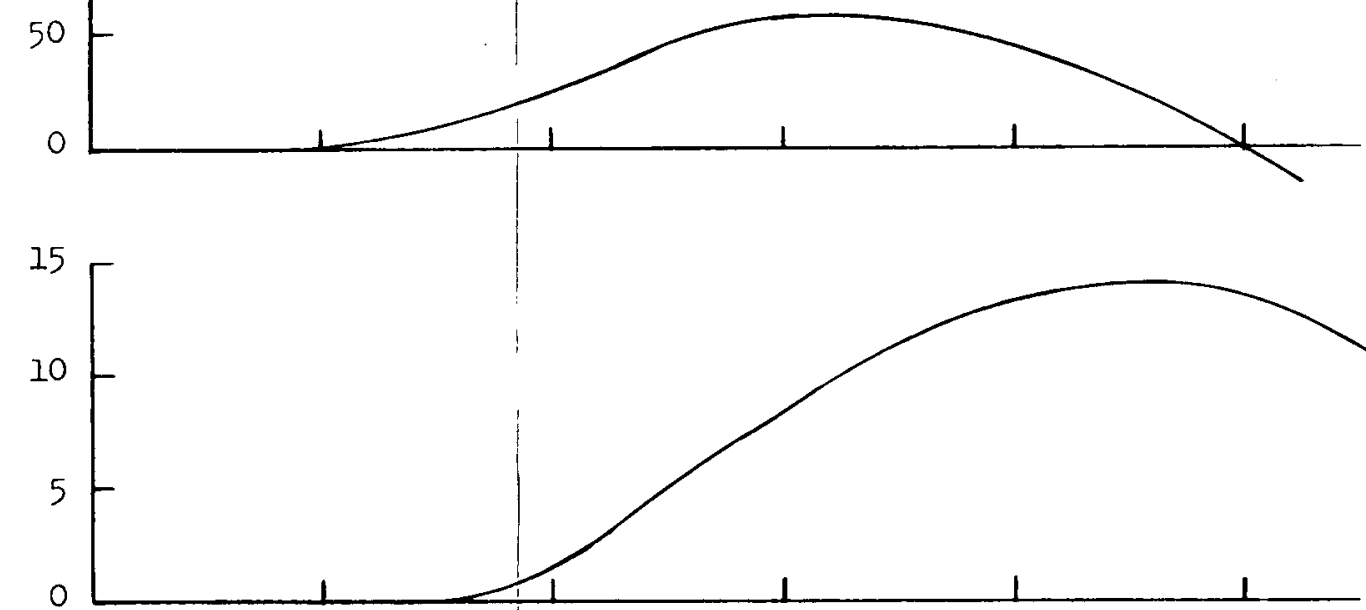

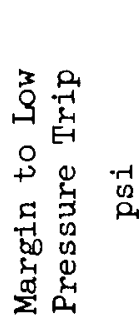

. 310
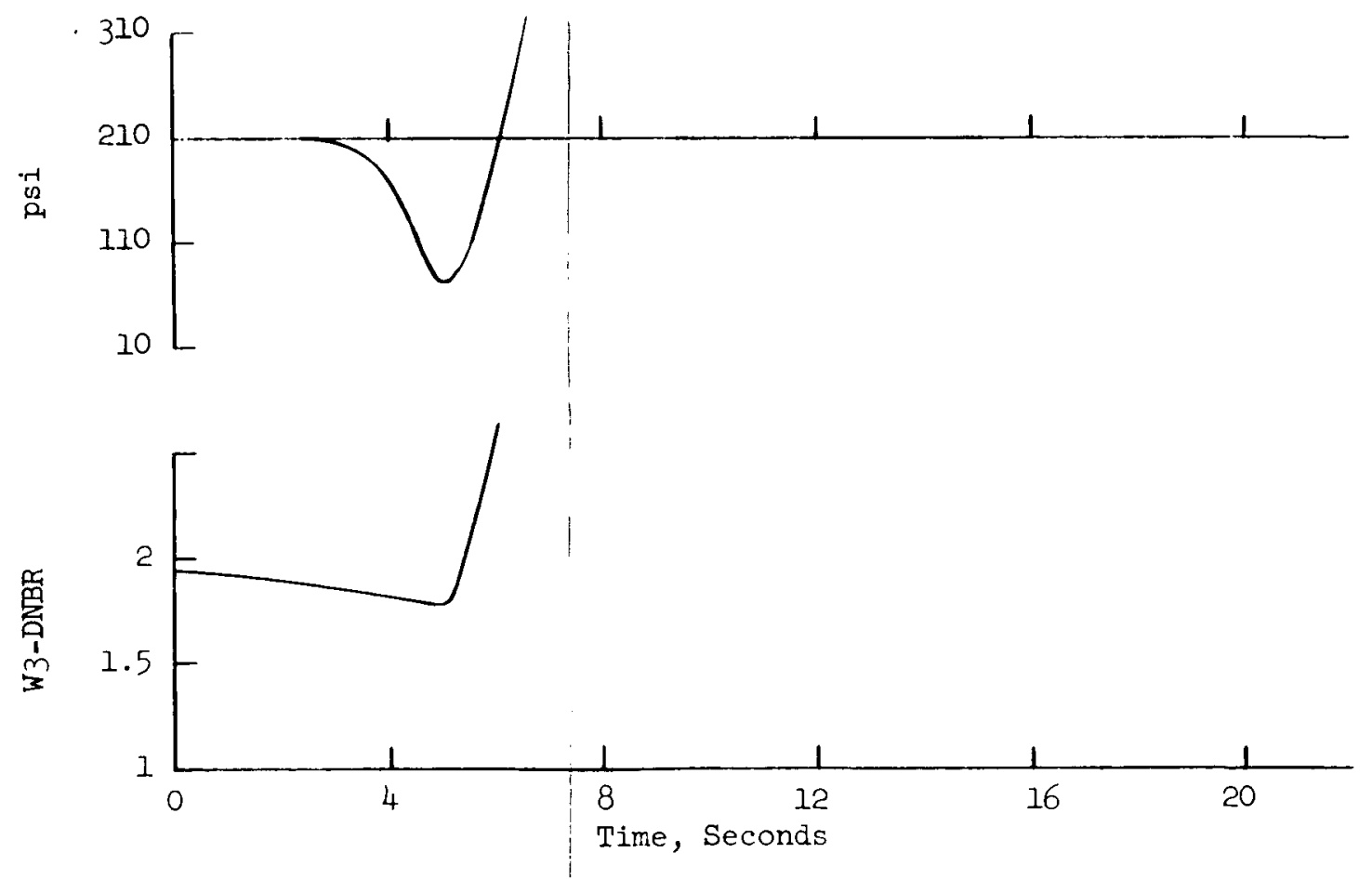

FIGURE 9.12

TRANSIENT RESPONSES FOR A FAST ROD

WITHDRAWAL ACCIDENT 


\subsubsection{FACILITY DESIGN ASPECTS (Continued)}

about five seconds and terminates the transient well before the coolant temperature and pressure have shown any increase. The rise in core inlet temperature and pressurizer pressure are a result of the turbine trip accompanying the reactor trip. Steam release to the atmosphere and condenser was not assumed. The DNB ratio barely changes during the transient. The initial margin to low pressure trip is conservatively assumed to be the maximum value at that power level including instrument errors. This yields the greatest temperature and pressure changes before a low pressure trip will occur.

Figure 9.13 shows the transient starting from the same initial conditions but with a much smaller reactivity insertion rate of $2 \times 10^{-5} \delta \mathrm{k}$ per second, a zero moderator coefficient of reactivity and a greater negative Doppler coefficient of $2 \times 10^{-5} \delta \mathrm{k}$ per degree Fahrenheit. For these conditions the transient is considerabiy lengthened and the reactor trip occurs from the variable low pressure trip signal. The effect of spray is seen in the reduction of the pressure rise at about 40 seconds. The surge rate then becomes too large for spray to handle alone and the pressure rises to the power operated relief valve setting and is then limited to 2,250 psia. There is a steady increase in core water inlet temperature until the low pressure trip occurs. For this case the minimum DNB ratio is slightly less than for the fast withdrawal and demonstrates the temperature pressure dependence of the correlation.

Figure 9.14 shows the transient starting from 80 percent power with $\alpha_{\mathrm{W}}=$ to.5 $\times 10^{-4} \delta \mathrm{k}$ per degree Fahrenheit, $\alpha \mathrm{f}=-1 \times 10^{-5} \delta \mathrm{k}$ per degree Fahrenheit and for the low reactivity insertion rate of $2 \times 10^{-5} \delta \mathrm{k}$ per second. Spray was assumed to be ineffective for this case. Here, as in Figure 9.13, the transient is lengthened and the trip cccurs from variable low pressure. The maximum pressurizer pressure reached is 2,250 psia where the relief valves are adequate to limit the pressure. There is a greater change in core inlet temperature than in Figure 9.13, but it started lower for the lower power level according to the programmed average temperature as a function of load. Here again, as for the slow transient, the minimum DNBR is similar to the full power case shown in Figure 9.13. Note, however, that the maximum instrumentation errors are assumed for the low pressure trip and the trip will occur in all probability much sooner. The transient also assumes no manual action although there would be alarms actuated for high average temperature, high $\Delta T$ across the steam generator, high pressurizer pressure and high pressurizer level before the trip occurs.

\section{2 .3 CONCLUSIONS}

Transient conditions within the plant which could occur as a result of an uncontrolled rod withdrawal are constrained by a combination of the overpower, variable low pressure and fixed high pressure reactor trips to prevent DNB in the core. This protection has been shown to be adequate for all combinations of plant parameters which may exist at the time of the transient. Three different transients have been presented showing the time behavior of the plant during a rod withdrawal. 


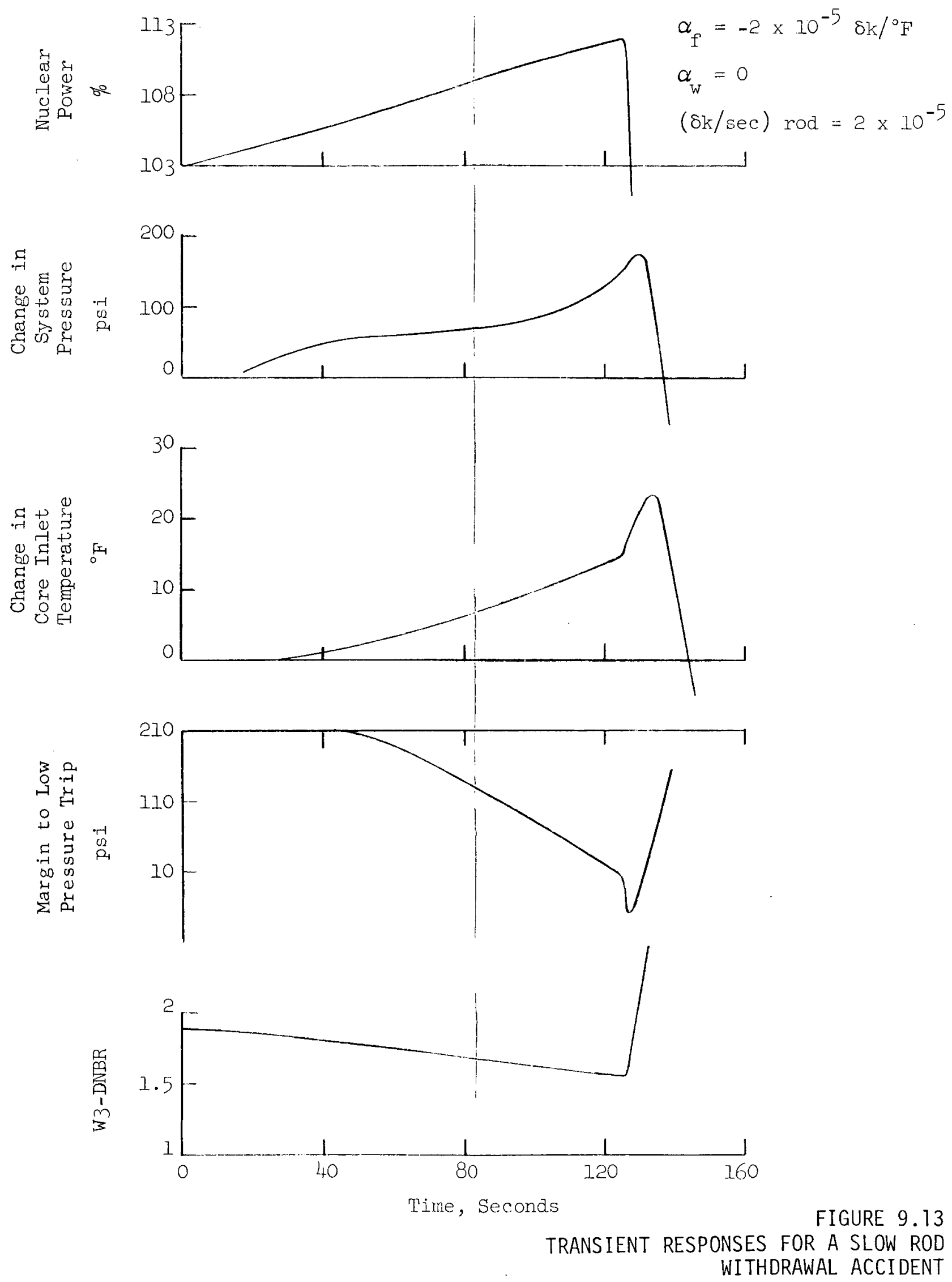




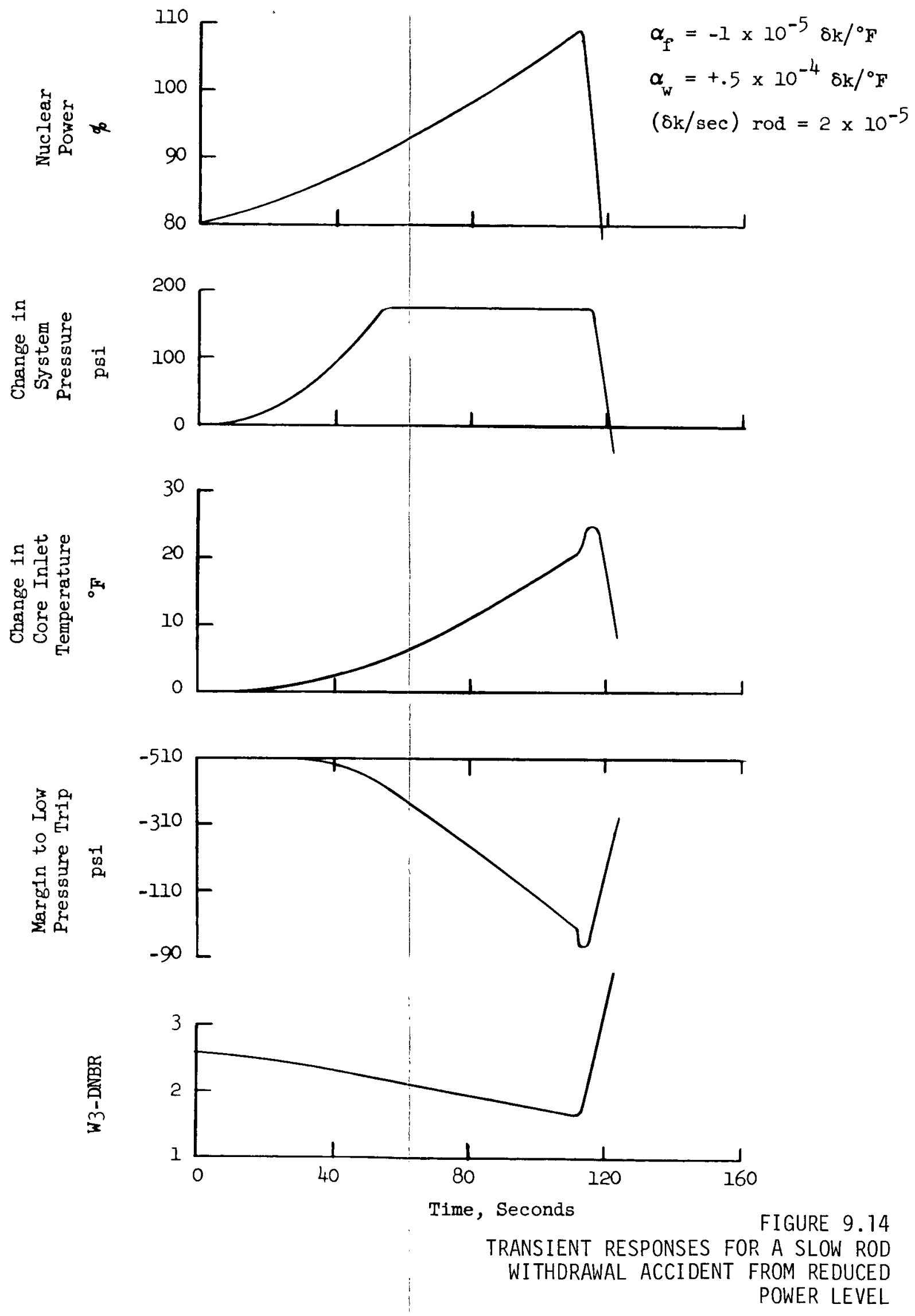




\subsection{BORON DILUTION}

\subsubsection{GENERAL}

Reactivity can be added to the reactor with the Chemical and Volume Control System by feeding a more dilute solution of boric acid into the Reactor Coolant System than is present in the reactor coolant. The possibility and consequences of causing an excessive reactivity insertion in this manner is considered in the design of the Chemical and volume Control System. To verify the adequacy of the design, the limiting cases of reactor transients which could result from improper boron dilution are analyzed.

In genera1, the protection against uncontrolled reactivity addition by boron dilution is the same as that described for control rod withdrawa1, the latter representing faster potential insertion rates. The maximum rate of boron dilution which can be attained with the Chemical and Volume Control System, Paragraph 3.6, results in a maximum reactivity insertion rate of less than $10^{-5} \delta \mathrm{k}$ per second. The Reactor Control and Protection System, Paragraph 6.2 , is designed to terminate reactivity insertion transients without core damage for all insertion rates up to $2 \times 10^{-4} \delta \mathrm{k}$ per second or 20 times the maximum boron dilution value.

\subsubsection{FACILITY DESIGN ASFECTS}

A detailed description of the means of achieving and controlling the desired blend of boric acid and demineralized water in the reactor coolant makeup cycle is included in Paragraph 3.6, Chemical and Volume Control system. As indicated in that description, the maximum rate of dilution is limited by the upper limit of the adjustable flow set point in the reactor makeup control.

\section{Manua1 Operation}

Three separate manual operator actions are required before the chemical and Volume Control System can be used to dilute the reactor coolant boron concentration by any amount. First, the operator must switch from the automatic makeup mode to the dilute mode. Second, the batching integrator must be set to some injection volume. Third, the start button must be depressed. Omitting any one step would prevent dilution. This makes the possibility of inadvertent dilution extremely small.

Information on the status of the reactor coolant makeup control is continuously available to the operator. Lights are provided on the control board to indicate the operating condition of valves and pumps in the chemical and Volume Control System. Alarms are actuated to warn the operator if boric acid or demineralized water flow rates deviate from the preset values as a result of system malfunction.

During operation of the reactor, control rod position indication gives a sensitive and direct measure of any changes in boron concentration. Dilution of $50 \mathrm{ppm}$ in boron concentration will require, on the average, a control group insertion of 20 inches to maintain a constant load and temperature. 


\subsubsection{FACILITY DESIGN ASPECTS (Continued)}

Improper Dilution

If the reactor is at power and under automatic control, the effect on shutdown reactivity margin of improper dilution would be control group insertion to maintain the desired average coolant temperature. The Reactor Contro1 and Protection System automatically actuates a rod insertion alarm signal at a programmed limit where an adequate shutdown margin is assured. This LO alarm alerts the operator to borate using the normal boration procedure for the Chemical and Volume Control System. The additional boron will result in reduction of the coolant average temperature and the control group will withdraw to maintain the programed temperature. If the dilution continues after this operation has been performed, the control group will continue to insert until a LO-LO alarm is actuated. At this point special procedures are initiated to inject borated water by either:

1) Aligning a boric acid transfer pump with the suction to the charging pump permitting a $45 \mathrm{gpm}$ flow of 12 percent boric acid solution from the boric acid tank.

2) Aligning the refueling water tank discharge with the suction of the charging pump to supply borated water at 2,900 ppm to the Reactor Coolant System.

Both of these operations can be conveniently performed directly from the control room.

If the reactor is at power and under manual control, boron dilution will result in a slow rise in reactor power and coolant temperature. This slow transient would be terminated by the variable low pressure reactor trip, assuming the control board indications and alarms on coolant temperature were ignored.

\section{Extended Boron Dilution}

If boron dilution is continued for an extended period of time, a loss of shutdown margin would result. In view of the numerous alarms and indications of the impending condition, and considering that an adequate shutdown margin is to be maintained during normal power operation, there is considerable time to evaluate and correct the situation by several alternate means and thus maintain a safe shutdown margin. Details of the alarms and indications are described in Paragraphs 3.6 and 6.2 .

During hot shutdown or cold shutdown conditions, greater shutdown is maintained, providing even more time to sense and correct any extended boron dilution effects. Under these shutdown conditions the boron concentration is periodically sampled. In addition, the source range channels will indicate any increase in neutron flux level. The rate at which boron dilution can occur with the Chemical and Volume Control System is very slow and any approach to criticality would occur at a much lower startup rate than in the case of the transient shown in Paragraph 9.1. 


\subsubsection{FACILITY DESIGN ASPECTS (Continued)}

Assumptions and Method of Analysis

The lower control group insertion limit for each control group bank is monitored by its own shutdown margin monitor. The allowable insertion is established as a function of the reactivity requirements for power coefficient and moderator coefficient with the programmed average temperature, as explained in Paragraph 3.4, Reactor Nuclear Design. Figure 3.28 in that section shows the lower insertion limit for bank two, for beginning of life. In this case bank one is fully withdrawn. As the core proceeds through life and as boron is removed from the reactor coolant, the moderator coefficient becomes more negative. Additional reactivity is required in control rods to handle the programmed average temperature as a function of load. Bank one is then used in addition to bank two to handle the hot to power reactivity variation. Figure 3.29 shows the lower insertion limit for banks one and two for end of life. The lower insertion limit is calculated as a linear function of measured average coolant temperature and of power, as determined by the average reactor coolant temperature differential. Thus any change in power or temperature, which could $1 \in$ ad to reduction in shutdown margin, is indicated by an increase in the lower insertion limit requiring the addition of boron to withdraw the control group. The parameters, in the shutdown margin monitors, will be periodically adjusted throughout lifetime to reflect the change in moderator coefficient. Failure to make these changes would yield a premature indication of loss of shutdown margin.

\section{$\underline{\text { Results }}$}

The normal dilution rate maintained with the Chemical and Volume control System is $45 \mathrm{gpm}$, but provisions are made to permit dilution at $90 \mathrm{gpm}$. Figure 9.15 shows the effective reactivity addition rate for these dilution rates as a function of reactor coolant boron concentration. This figure includes the effect of increasing boron worth in reactivity at lower boron concentrations. These rates are obtained for an average coolant temperature of $578 \mathrm{~F}$.

At lower temperatures the boron worth is greater yielding a somewhat greater reactivity insertion rate as indicated by the point shown for a $\mathrm{T}_{\text {avg }}$ of $78 \mathrm{~F}$ and 2,500 ppm boron. Even for this condition the maximum reactivity insertion rate is less than $10^{-5} \delta \mathrm{k}$ per second.

Paragraph 3.4 summarizes the control rod requirements for shutdown. It is shown that at beginning of core life there is 2.9 percent shutdown with the highest worth rod stuck out including a 20 percent allowance for uncertainties. The shutdown at end of life is 2.2 percent. Therefore, at beginning of life, where the reactivity insertion resulting from boron dilution is greatest, it would require continuous dilution at the maximum rate of 90 gpm for almost 50 minutes at a conservatively high dilution reactivity rate of $10^{-5}$ $\delta \mathrm{k}$ per second to lose the 2.9 percent shutdown.

With the reactor under manual control the reactivity insertion at $10^{-5} \delta \mathrm{k}$ per second would result in a slow rise in coolant temperature and core power similar to the slow rod withdrawal transient until the variable low pressure trip is actuated. Figures 9.13 and 9.14 , in conjunction with the control rod withdrawal at power, demonstrate the protection provided by the low pressure 


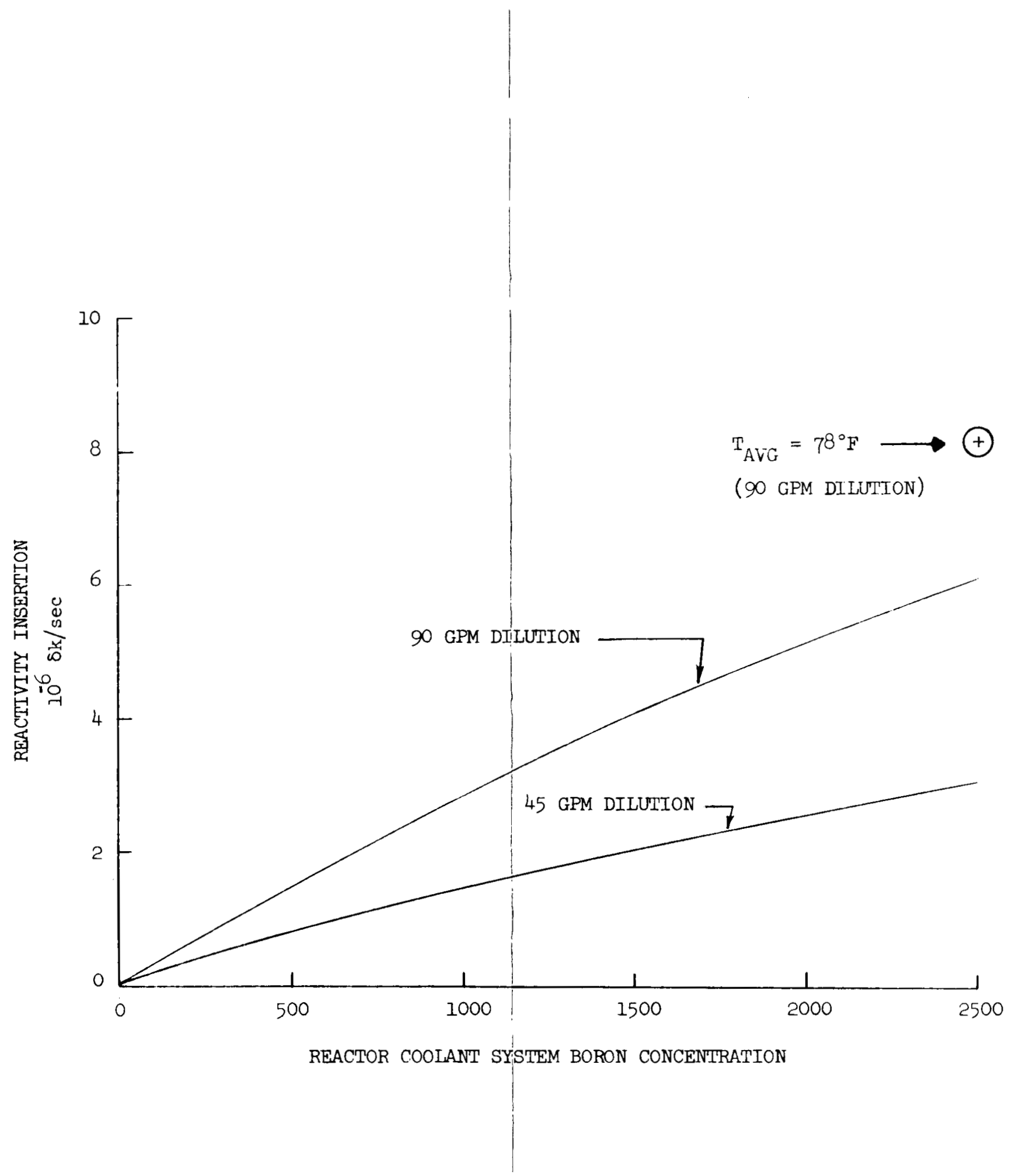

FIGURE 9.15 REACTIVITY INSERTION RATE FOR BORON DILUTION AT $T_{A V G}=578 \mathrm{~F}$ 


\subsubsection{FACILITY DESIGN ASPECTS (Continued)}

trip for this very slow insertion rate. The trip would occur within two to three minutes starting from full power operation.

As shown in Paragraph 3.4, for the all rods in condition, there is an additional one percent shutdown. Therefore, for the hot shutdown or cold shutdown condition there is a possible additional dilution time of about 17 minutes, in addition to the previously mentioned 50 minutes, before criticality is reached.

\section{3 .3 CONCLUSIONS}

The maximum boron dilution rate achievable with the Chemical and Volume Control System is limited by design to a rate very small with relation to the ability of the control rods to change reactivity. Excess shutdown reactivity is maintained at all times by the prescribed limits on control group insertion and two alarms are provided to alert the operator when these limits are reached. Numerous alarms and information are provided to alert the operator to the performance and status of the Chemical and Volume Control system. The excess shutdown, coupled with the inherent limit on reactivity insertion by dilution, provide the operator with adequate time to add boron as necessary by one of several alternate means to maintain shutdown margin.

The core is protected by the variable low pressure trip if boron dilution continues at power without compensating control group insertion. 


\subsection{COLD WATER}

\subsubsection{GENERAL}

The addition of cold water to the core will result in an insertion of positive reactivity. The magnitude of the insertion is dependent upon the value of the negative moderator coefficient of reactivity. In a chemical shim plant the maximum value of the negative moderator coefficient is reduced due to the presence of boric acid dissolved in the coolant, thereby decreasing the potential reactivity insertion resulting from a cooldown. The most severe condition for a cold water transient in a chemical shim plant exists at the end of core life when the Reactor Coolant System is essentially boron free and the most negative moderator coefficient exists.

Three potential means of rapidly reducing the core water inlet temperature are considered in evaluating core protection against this type of reactivity insertion. They are:

a. Startup of an inactive loop

b. Addition of excess cold feedwater to the steam generators

c. Sudden increase in steam discharge from the steam generators

Within the capability of the Reactor Control and Protection System, these transients can be accommodated without trip. For more severe cases, reactor protection in the form of the variable low pressure and overpower trips will terminate the transients without core damage.

\subsubsection{STARTUP OF AN INACTIVE LOOP}

\section{Facility Design Aspects}

Operation of the plant at power with a reactor coolant loop out of service (i.e., the reactor coolant pump shut down) results in a decrease in the coolant temperature in that loop. A subsequent restart of the idle reactor coolant pump without bringing that loop closer to system average temperature would then inject colder water, mixed with the flow from the active loops, into the core. The resultant reactivity insertion could exceed that which the Reactor Control System is able to follow. In the same manner as described in Paragraph 9.2 for rapid reactivity additions by control rod motion, the overpower trip will terminate the transient before the limiting margin to DNB is reached.

The plant has no valves to isolate a reactor coolant loop, therefore, operation of two reactor coolant pumps will result in backflow through the idle loop, in turn causing a reverse $\Delta \mathrm{T}$ in that loop, i.e. the temperature of the normal hot leg falls below that of the reactor inlet. A temperature interlock and alarm is provided for each loop to prevent starting a reactor coolant pump in an inactive loop if a reverse $\Delta T$ exists greater than a preset value. Restarting an idle loop can be accomplished by reducing load, 


\subsubsection{STARTUP OF AN INACTIVE LOOP (Continued)}

which reduces the $\Delta T$ in the active loops to a value which will allow the idle loop pump to start.

Since there is no means of isolating a steam generator, the maximum idle loop temperature depression (with very small 1 backflow) is 1 imited by the temperature of the turbine cycle, thus providing an inherent limit on the severity of the transient. The range of possible inverse $\Delta T$ conditions is determined for a range of predicted backflow rates, and an analysis of the core response is performed for the most severe case assuming the $\Delta T$ interlock to fail.

\section{Assumptions and Method of Analysis}

The transient following startup of an inactive loop is calculated with a detailed plant simulation on an analog computer. A conservative calculation is made for low backflow and a conservative estimate of the overall heat transfer coefficient for the idle steam generator is made. These conditions yield the maximum idle loop temperature depression and the maximum initial power with two out of three loops. The reverse $\Delta \mathrm{T}$ interlock and alarm will prevent a restart of the idle loop until the temperature difference is less than $5 \mathrm{~F}$. For this transient the interlock is assumed to be inoperative. The pump is assumed to accelerate to full flow instantaneously and the entire idle loop hot leg at the reduced temperature is swept into the core. A conservative maximum negative moderator coefficient is assumed. As a limiting condition the cold water is assumed to reach the core without mixing with the active loop flows, but is assumed to mix with the water in the inlet plenum. The core response is calculated assuming the temperature change to appear throughout the core. A low value of Doppler coefficient is assumed thus yielding the maximum power increase as a result of the cold water.

For the purposes of this transient calculation, the ratio of power level to coolant flow through the core is assumed to be a constant, since this yields the minimum steam pressure, and the greatest possible temperature decrease in the inactive loop. This assumption also results in the smallest initial margin to DNB. A high heat transfer coefficient from reactor cycle to turbine cycle in the inactive loop is used. This is approximately 85 percent of its normal full load value, whereas, the heat transfer coefficient will decrease a greater amount at the low load level at which the inactive loop is operating - approximately 18 percent of its normal full load value.

\section{$\underline{\text { Results }}$}

A backflow rate of $20,800 \mathrm{gpm}$ in an idle loop was determined for operation with two out of three reactor coolant pumps running. The flow rate through the active loops is $74,500 \mathrm{gpm}$ each, an increase over the nominal flow with three loop operation. The resulting total core flow is then 128,200 gpm or about 65 percent of normal three loop flow. The resulting cold leg temperature in all loops is $542 \mathrm{~F}$ and the hot leg temperature in the inactive $100 \mathrm{p}$ is $505 \mathrm{~F}$. The steam generator temperature in the inactive loop is $500 \mathrm{~F}$ so the inactive loop is approaching its minimum possible value using the high heat transfer coefficient. The hot leg temperature in the idle 


\subsubsection{STARTUP OF AN INACTIVE LOOP (Continued)}

loop cannot go below the steam temperature. The assumed Doppler coefficient is $-1 \times 10^{-5} \mathrm{k}$ per $\mathrm{F}$ and the moderator is $-3.5 \times 10^{-4} \mathrm{k}$ per $\mathrm{F}$. The transient results shown in Figure 9.16 are computed assuming the ful1 $37 \mathrm{~F}$ temperature decrease is imposed at the inlet to the reactor vessel. Credit is taken for a mixing delay in the reactor inlet plenum but no credit for flow mixing with the two active loops.

The peak nuclear power occurs in about eight seconds and a peak thermal power of about 85 percent is reached. The reactor trip is from overpower at 100 percent. This is a conservative high value since the overpower trip at this power level would be set to give a maximum trip level of 80 percent of full power.

Since the peak thermal power is less than full power and the inlet temperature is less than the ful.I power value there is margin to DNB. The minimum DNB ratio is 3.1 and occurs at about 10 seconds.

Figure 9.17 shows the effect of flow mixing with the active loops. The temperature change at the reactor vessel inlet plenum then becomes $-12 \mathrm{~F}$ instead of $-37 \mathrm{~F}$. All other parameters are identical to those for the transient shown in Figure 9.16. The large reduction in transient peaks is evident from the two figures. The actual transient would lie somewhere between the two results and would most likely be represented by the results which assume flow mixing.

\subsubsection{ADDITION OF EXCESS FEEDWATER}

\section{Facility Design Aspects}

The addition of excess feedwater to a steam generator is also analyzed. The effect of the additional feedwater is similar to that of a load increase, causing a reduction in cold leg temperature of the affected loops. The temperature response to the cold feedwater is attenuated by the thermal capacity of the Reactor Coolant and Steam Systems.

Reactor control responds to the load increase within the transient capability of the plant. Since reactor control is based on Reactor Coolant System conditions and core power, it does not distinguish between a true load increase and a feedwater addition transient. If reactor control is unable to maintain plant conditions within the protection limits during the transient, the overpower or variable low pressure protection will cause a reactor trip without core damage.

\section{Assumptions and Methods of Analysis}

The addition of excess feedwater is also analyzed by means of a detailed analog simulation. All three feedwater control valves are assumed to fully open together at full load. For purposes of analysis a complete loss of the air supply to the val.ves was assumed causing them to open. Since there are three instrument air compressors, providing a highly reliable air system, this occurrence is considered very improbable. However, this case was 

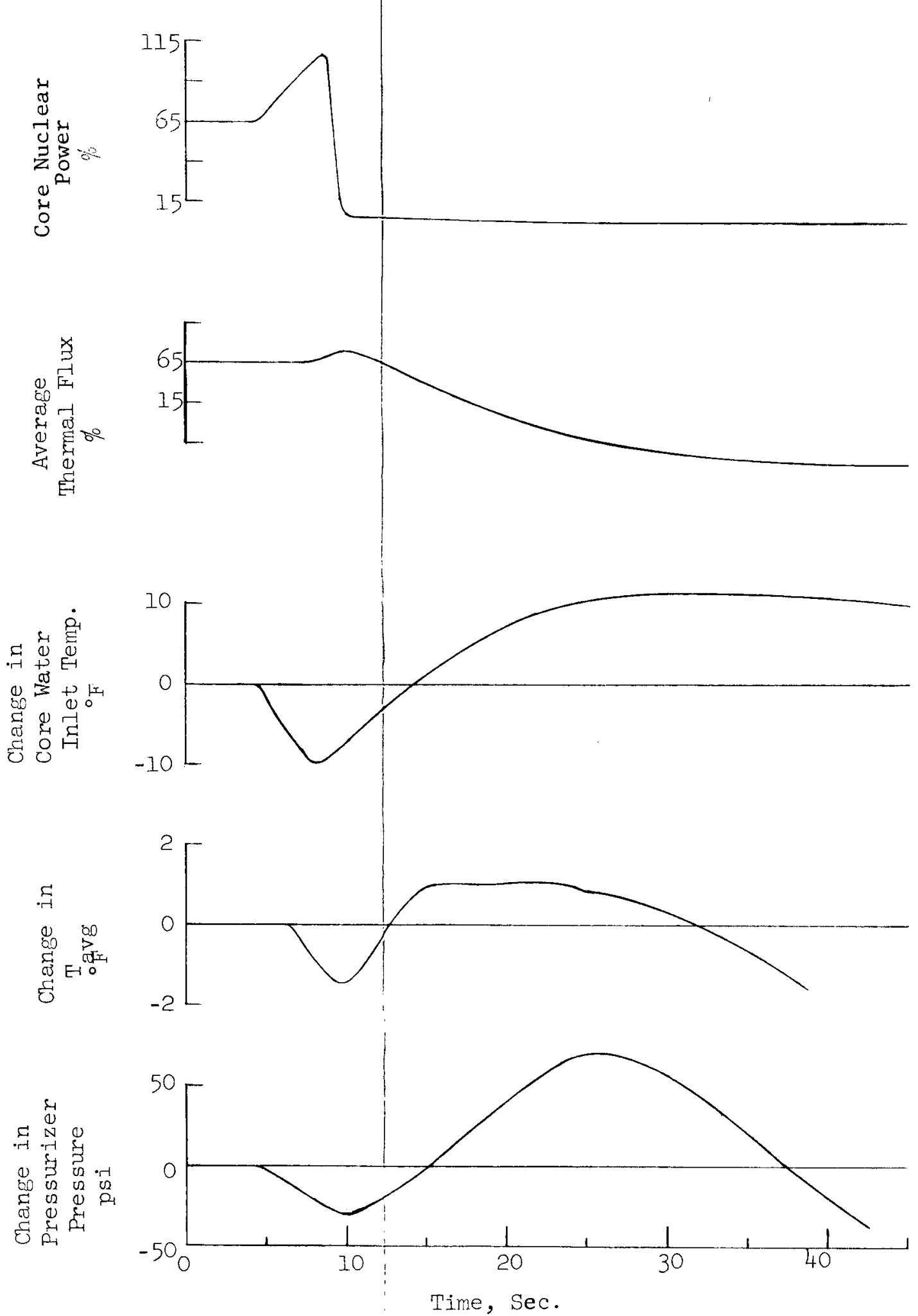

FIGURE 9.16 TRANSIENT RESPONSE FOR STARTUP OF AN INACTIVE LOOP WITHOUT FULL TEMPERATURE DECREASE 

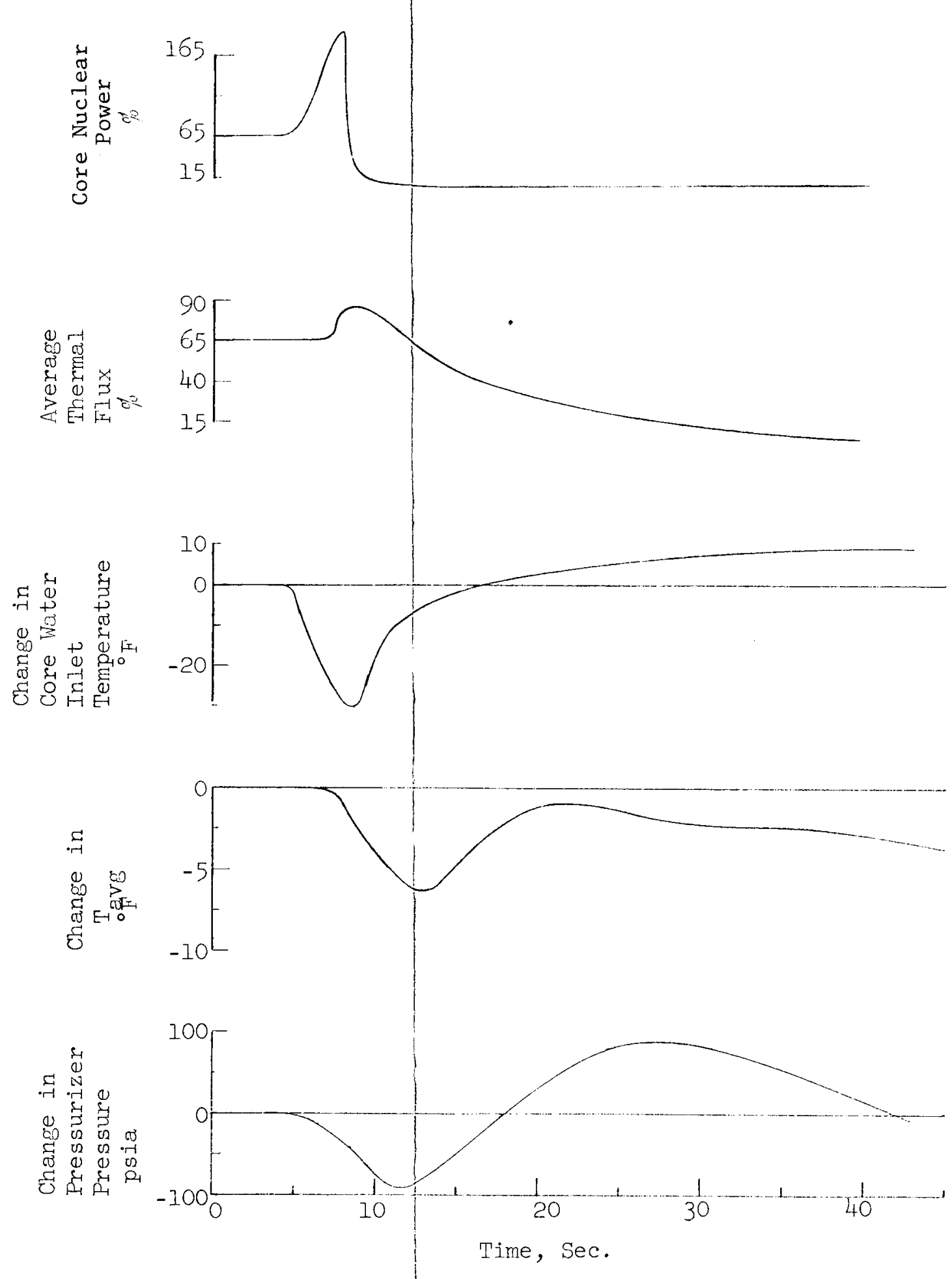

FIGURE 9.17

TRANSIENT RESPONSE FOR STARTUP OF AN INACTIVE LOOP WITH FULL TEMPERATURE DECREASE 


\subsubsection{ADDITION OF EXCESS FEEDWATER (Continued)}

investigated to determine the effect on the core integrity. A 1 arge negative moderator coefficient is assumed which yields the maximum reactivity insertion. The transient is analyzed at full load where the additional available feedwater flow is limited by the feedwater system head-flow characteristics and at lower loads with only one feedwater pump running. For this case the transient is studied assuming the second pump is started and that all feedwater valves are in manual control.

\section{$\underline{\text { Results }}$}

The most severe case of excess feedwater addition while at full power was analyzed. That is the sudden opening of all feedwater valves to their wide open position. The moderator coefficient of reactivity was assumed equal to $-3.5 \times 10^{-4} \delta \mathrm{k}$ per $\mathrm{F}$ and the Doppler to be $-1 \times 10^{-5} \delta \mathrm{k}$ per $\mathrm{F}$. No credit is taken for reactor control response. As seen in Figure 9.18 even for these very conservative assumptions in the first two minutes of the transient, prior to a trip of the unit, there is no significant reduction in core inlet temperature and there is very little response to this condition by the Reactor Coolant System at all. The first indications of the situation are high level alarms on the steam generators to alert the operator and it would be about two minutes before the upper level indicator is reached. It was assumed for this case that a manual trip is made at about two minutes.

Automatic steam release to condenser and atmosphere is neglected as a heat sink for this case. Following the turbine trip the steam generator pressure rises to the safety valve setting in about 10 seconds, thus Iimiting the peak core inlet temperature.

The transient behavior for startup of a feedwater pump with one running while at 50 percent power is shown in Figure 9.19. Al1 three feedwater valves were assumed to be in manual control, a very remote possibility at this power leve1. The same Doppler and moderator coefficients were assumed as for Figure 9.18. The transients as shown in Figure 9.19 are very smal1.

The three steam generator high level alarms will annunciate within seconds from the time the pump was started. It is assumed that a manual trip is initiated 40 seconds from the time the feedwater pump was assumed to be started.

The Reactor Coolant System has essentially reached an equilibrium condition as this time with very little deviation from initial conditions as shown by the dashed lines in the figure showing coolant conditions without a trip. The core never approaches a DNB limit during this transient.

\subsubsection{LARGE LOAD INCREASES}

\section{Facility Design Aspects}

The Reactor Control and Protection System is designed to accommodate step load increases of the order of 10 percent without a reactor trip. A 

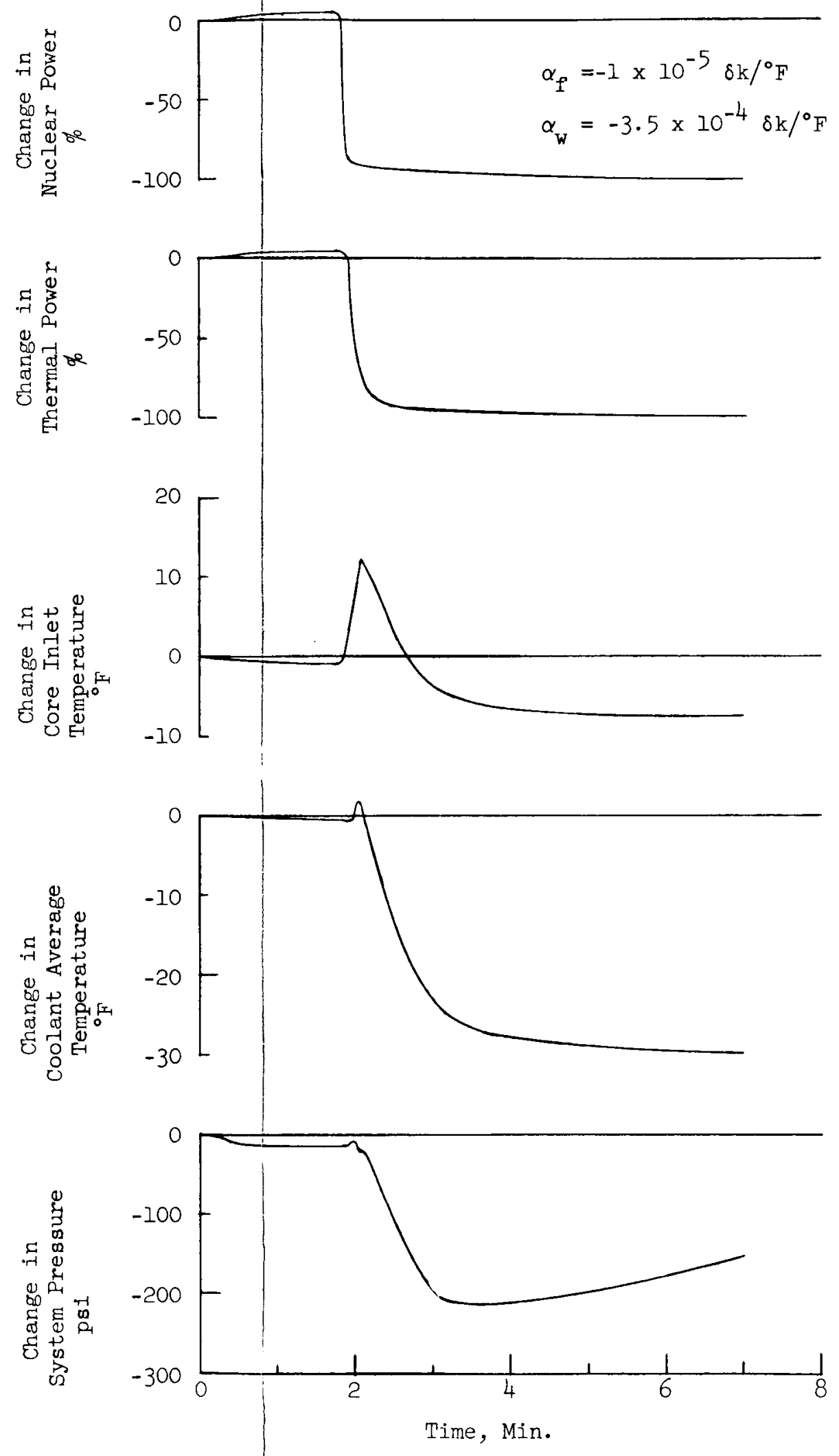

FIGURE 9.18

TRANSIENT RESPONSES FOR ALL THREE FEEDWATER VALVES FULL OPEN 

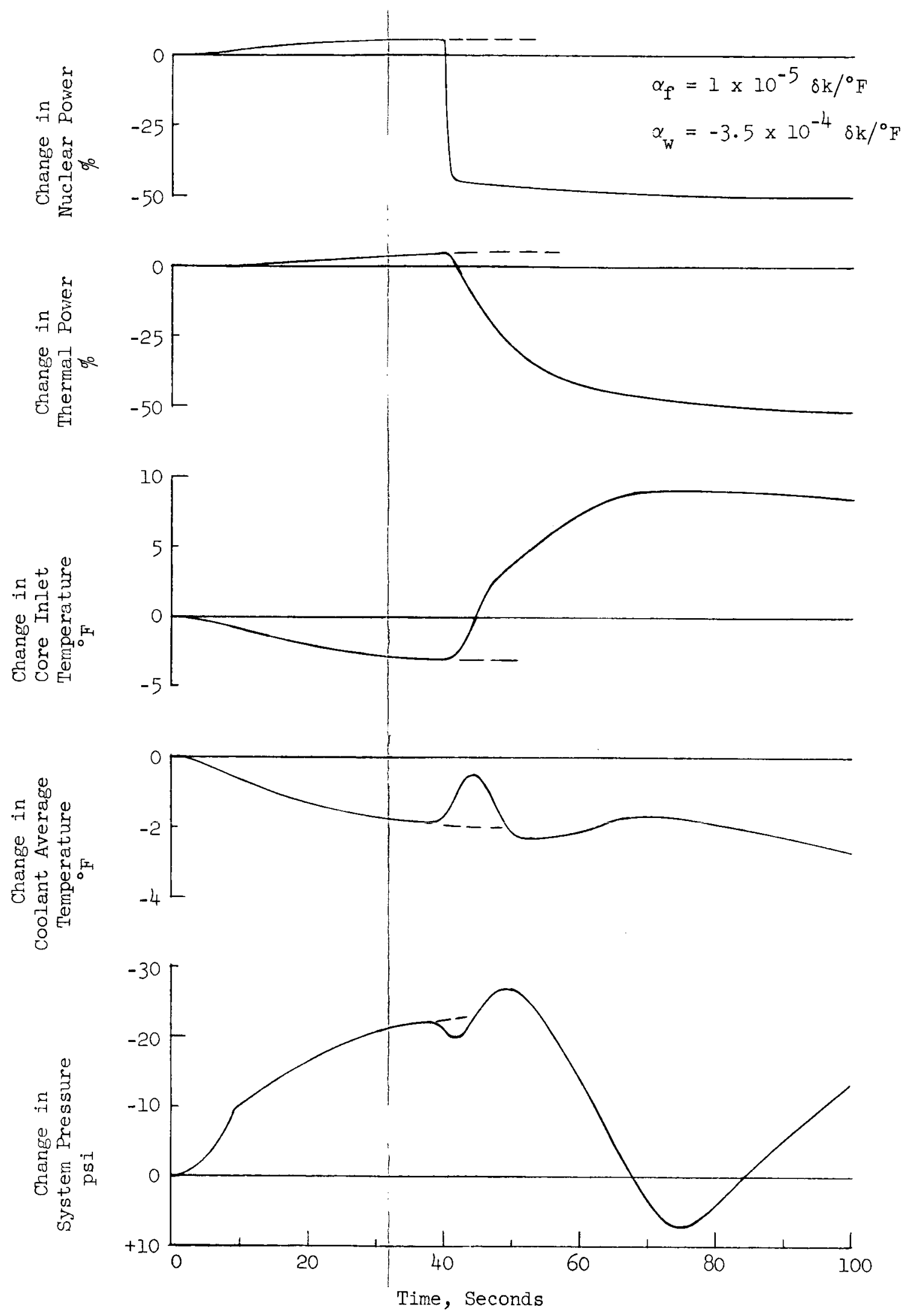

FIGURE 9.19 TRANSIENT RESPONSES FOR STARTUP OF THE SECOND FEEDWATER PUMP 


\subsubsection{LARGE LOAD INCREASES (Continued)}

load tracking device is provided as a part of the turbine load limit control to maintain the load limit a preset amount above operating load but not to exceed rated full load. Any sudden load pickup from the turbine governor control is limited to this preset amount. At the end of core life this transient capability is increased because of the more negative moderator coefficient. Very large load increases, however, would cause an excessive reduction in reactor coolant temperatures, a large coolant contraction and resultant low pressure. The core is protected from DNB during this transient by the overpower-low pressure protection described in Paragraph 9.2. For the very large apparent increase in turbine cycle load caused by a steam line break this protection is provided during the initial transient. The behavior of the reactor during the steam line break is discussed in Paragraph 10.4 .

\section{Assumptions and Method of Analysis}

The turbine generator load limit with the automatic tracking feature will limit, any load increase introduced either mantally or automatically by turbine-governor control. Very large load increases, beyond the capability of the Reactor Control and Protection System, are protected by the combination of the variable low pressure trip and the nuclear overpower trip as described in Paragraph 9.2. Transient conditions following any load change will be constrained within the limits shown in Figure 9.8. If the reactor control is unable to maintain the plant conditions within these constraints following a load increase, a reactor trip will occur and no DNB can occur. The analysis presented here is determined from a detailed analog plant simulation and illustrates the operation of the reactor control and the automatic load tracking by the turbine load limit for a large step increase in load. A more severe pressure and temperature transient occurs in this case with a positive moderator coefficient, since a negative moderator coefficient serves to initially increase the reactor power leve 1 in response to a turbine load increase. With either a positive or negative coefficient the automatic control system must withdraw rods, to compensate for Doppler and moderator, to increase load and average temperature according to the temperature program. For the positive coefficient the withdrawal will be initiated earlier. A low incremental control group worth is also assumed.

\section{$\underline{\text { Results }}$}

Figure 9.20 shows a transient response to a sudden requirement for 30 percent more load by the turbine governor control while operating at 70 percent load. The reactor control is assumed to be in automatic. A maximum positive moderator coefficient of $+0.5 \times 10^{-4} 8 \mathrm{k}$ per $\mathrm{F}$ and a low Doppler of $-1.2 \times 10^{-5} \delta \mathrm{k}$ per $\mathrm{F}$ are assumed which yield the most severe transient. The load tracking control is assumed to be set to allow a sudden 10 percent increase in load and it will then increase at a rate of 25 percent per minute to the desired load. The resulting transient is handled without a reactor trip and without excessive temperature or pressure excursions as shown in the figure. The increase of $14 \mathrm{~F}$ in $\mathrm{Tavg}$ is to maintain the temperature program as a function of load. 

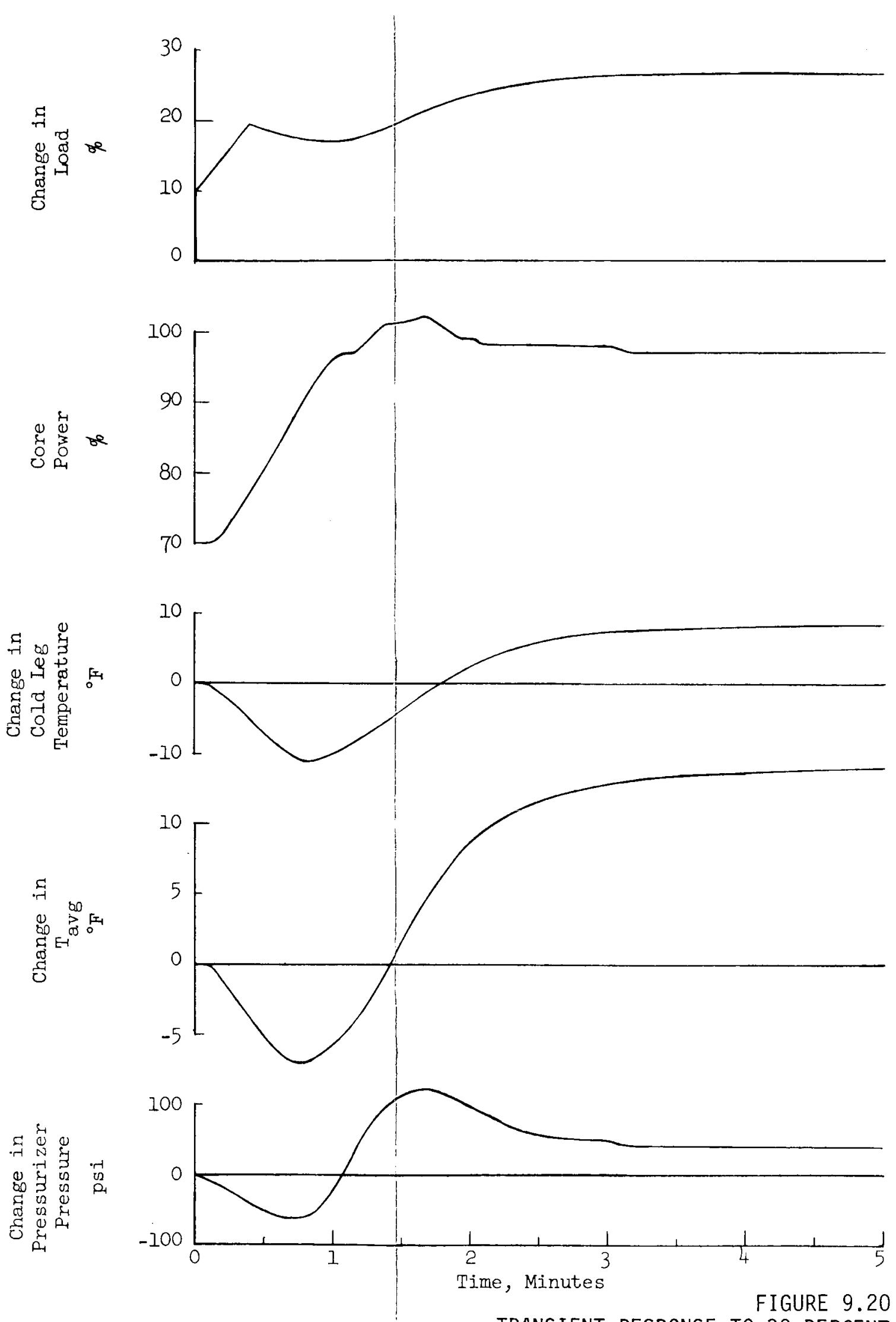

FIGURE 9.20

TRANSIENT RESPONSE TO 30 PERCENT STEP LOAD INCREASE WITH LOAD LIMIT

TRACKING 


\subsubsection{LARGE LOAD INCREASES (Continued)}

Under manual control and without operator intervention, the coolant temperature and pressure, in the case of the positive moderator coefficient, would continue to decrease until the variable low pressure trip is reached. The core is protected as shown in Figure 9.22.

With a negative moderator coefficient, a steady state condition may be achieved at a lower average temperature since the reduction in temperature will increase reactor power. This temperature reduction may also result in a large pressurizer pressure decrease and result in a low pressure trip.

\subsubsection{CONCLUSIONS}

Various means of introducing cold water into the core have been examined for end of life core conditions with the maximum negative moderator coefficient. There are no means of causing large and sustained temperature reductions at the core inlet with the exception of the assumed large steam line break described in Paragraph 10.4. The Reactor Control and Protection System will protect the core from DNB for other cold water transients associated with startup of an inactive loop or excess feedwater addition. 


\subsection{DROPPED ROD}

\subsubsection{GENERAL}

If one control rod should be released and drop into the core, the immediate effect would be a reduction of reactivity and a corresponding decrease in reactor power. Returning the reactor to normal power without withdrawing the dropped rod could result in local power densities in excess of design limits due to the distorted power distribution.

\section{Protective Circuits}

Two separate protective circuits are provided, each performing two separate functions, to preserve safe operating conditions following a rod drop. The first is to block automatic rod withdrawal, and the second is to initiate a turbine load cutback to a safe level.

\section{Mode of Detection}

These two functions are initiated by either of two independent signals: one signal from the nuclear instrumentation and the other signal from the rod position indicators. Either mode of detection is sensitive enough to respond to a rod drop at substantial power levels, and either mode of protection is adequate to prevent DNB. If only the rod withdrawal block function were actuated, the reactor power could reach equilibrium at a lower value. While the minimum steady-state DNB ratio would be reduced in this case, the variable low pressure reactor trip would still maintain core protection. If only the turbine load cutback were actuated the maximum reactor power would be limited to a safe value consistent with the worst dropped-rod nuclear flux distribution.

\subsubsection{FACILITY DESIGN ASPECTS}

The probability of a rod cluster control assembly accidentally dropping is considered remote. The rod cluster control assemblies are positioned in the core by means of magnetic jack, hermetically sealed, rod drive mechanisms which are described in Paragraph 6.3 .

However, should a rod cluster control assembly drop into the core, the rod drop protection circuit and alarm are actuated from a rod bottom signal in the rod position indication system (Paragraph 6.4). The negative reactivity that would be inserted by a dropped rod will also cause a sudden decrease in neutron flux at one or more of the four power range nuclear instrumentation channels (Paragraph 6.1). The number of channels affected depends on the location of the dropped rod in the core. A flux difference circuit associated with each power range channel will initiate a rod drop alarm and actuate the rod drop protection circuit when this sudden decrease exceeds a preset amount. The flux difference set point will be 10 to 15 percent based on initial operating tests. The rod drop protection blocks automatic rod withdrawal and initiates a turbine load cutback to a preset load. This cutback is accomplished by turbine load limit rundown which 


\subsubsection{FACILITY DESIGN ASPECTS (Continued)}

overrides the governor valve control. The power cutback occurs at the rate of approximately 50 percent per minute.

To insure that the load cutback nearly matches the core power reduction resulting from the maximum negative reactivity insertion the final load for the power cutback is set according to the highest worth rod. This setting limits the severity of the reactor coolant temperature reduction, and hence pressure reduction, which could lead to a low pressure trip for a dropped rod while operating at or near full load. The power cutback reduces load to a preset absolute value. Should a dropped rod occur from a power level below the preset value, no cutback will occur. The core remains protected, however, since the plant is operating at a power level below the cutback value. The operator must reduce load manually to compensate for the core power reduction to prevent a reactor trip. If the load is not reduced, the mismatch between core power and turbine load will result in a reactor coolant temperature and pressure reduction and a low pressure trip.

Should a dropped control rod insert less negative reactivity than the highest worth rod when the plant is at full load, the turbine load cutback will exceed the core power reduction at that time resulting in an increase in Reactor Coolant System temperatures and pressure. The Reactor Control and Protection System responds to insert the control group and restore equilibrium conditions at the reduced load. For a large difference between the core power reduction and the turbine load cutback, a short term steam release to the atmosphere and condenser may occur.

The highest worth rod is determined in the course of startup experiments and the power distribution effects verified. The protection is based on the fact that the maximum power tilt, resulting from a dropped rod, does not exceed the difference in load required for the highest worth rod. The power cutback required for the highest worth rod is therefore adequate to protect the core for the power tilt associated with any dropped rod. The exact magnitude of turbine load cutback is then set and is expected that the reduced set point will be between 75 and 85 percent of full load.

\section{Assumptions and Method of Analysis}

The transient following a dropped rod is determined using a detailed analog simulation of the plant. The dropped rod is assumed to cause a step decrease in reactivity and the core power generated is determined using a pointwise neutron kinetics model. The overall plant response is calculated by simulating the turbine load runback and blocking of automatic rod withdrawal. A low incremental worth $\left(-2.5 \times 10^{-4} 8 \mathrm{k}\right.$ per inch) is assumed for the control group as equilibrium conditions are restored. The analysis is made for the case where the power cutback is nearly equal to that required by the negative reactivity insertion from the rod $\left(-2.5 \times 10^{-3} \delta \mathrm{k}\right)$ and for the case where the cutback is greater than required $\left(-1.25 \times 10^{-3} \delta \mathrm{k}\right)$.

\section{$\underline{\text { Results }}$}

Figure 9.21 illustrates the transient following a dropped rod worth $-1.25 \mathrm{x}$ $10^{-3} \mathrm{k} \mathrm{k}$ while operating at full power. For conservatism, the power cutback 

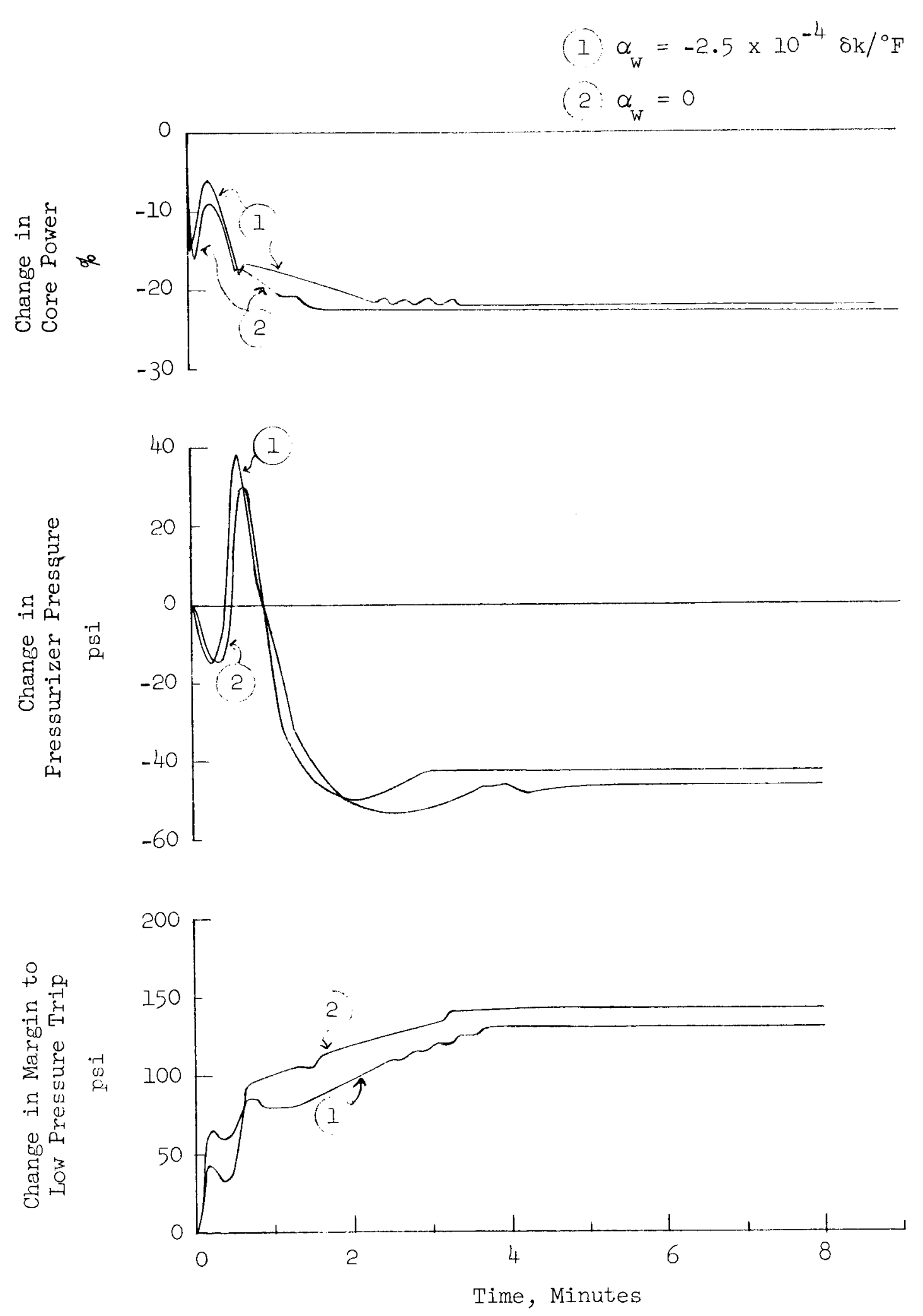

(1) $\alpha_{w}=-2.5 \times 10^{-4} 8 \mathrm{k} /{ }^{\circ} \mathrm{F}$

(2) $\alpha_{w}=0$
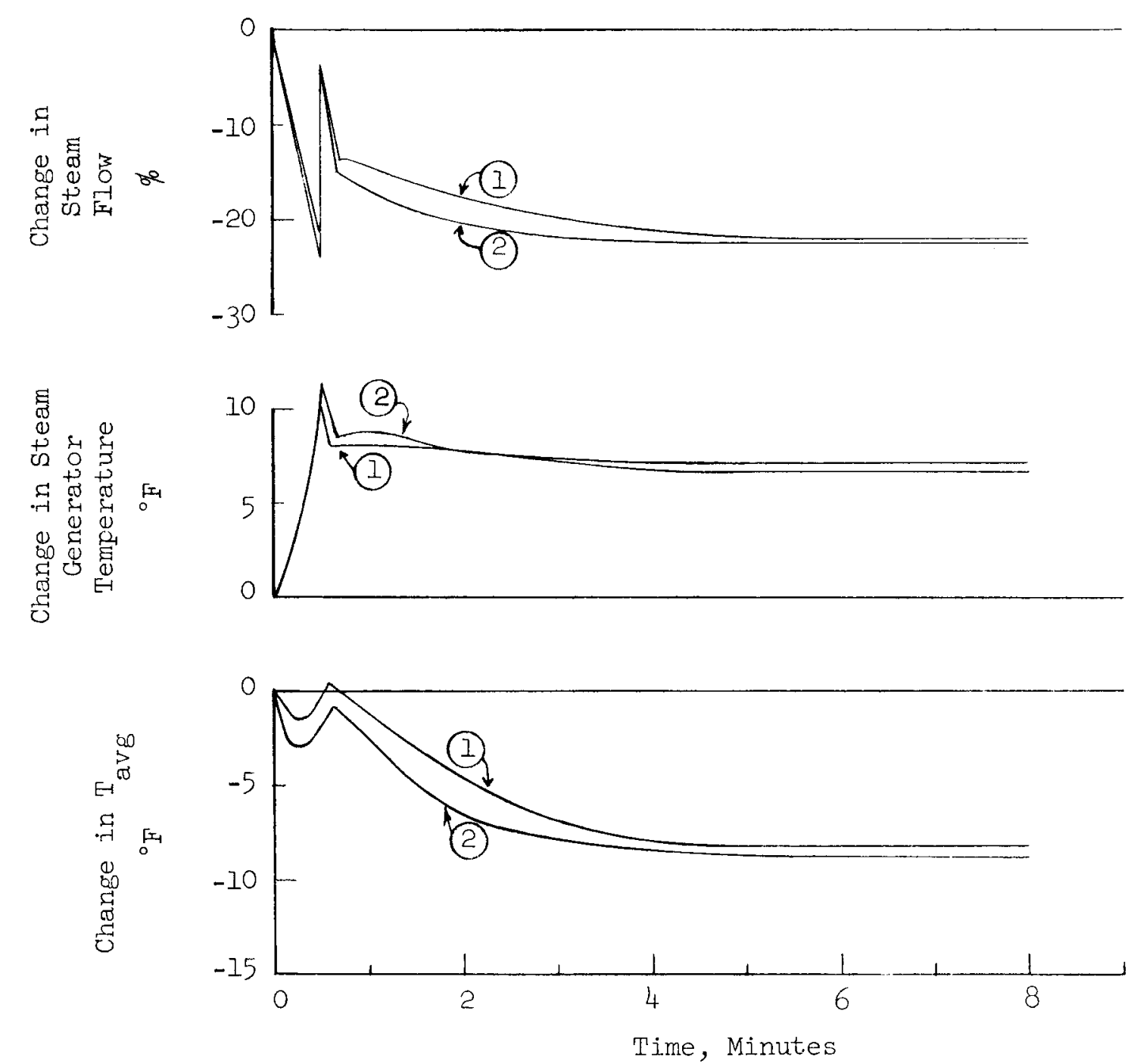

RESPONSE TO A DROPPED ROD RESPONSE $10^{-3}$ A OK WITH POWIR CUTBACK - $1.25 \times 15$ T $3 K$ WITH POUER CUTBACK TO 75 PERCENT LOAD, INCREMENTAL
CONTROL GROUP WORTH $=-2.5 \times 10^{-4}$ $\delta \mathrm{k} / \mathrm{INCH}$ 


\subsubsection{FACILITY DESIGN ASPECTS (Continued)}

is assumed to reduce load to 75 percent at a rate of 25 percent per minute. This load cutback is greater than required for the dropped rod worth which gives a prompt reduction of about 15 percent in core power. Consequently, the coolant average temperature begins to increase. The programmed average temperature setpoint is reduced to the value corresponding to the reduced load. Consequently, there is a short term automatic steam release caused by the large temperature error signal and the equilibrium temperature is achieved under automatic control rod insertion in about four minutes. There is no rod withdrawal during the transient. The two curves compare typical beginning of life and end of life transients with a zero moderator coefficient and a negative moderator coefficient of reactivity of $-2.5 \mathrm{x}$ $10^{-4} \delta \mathrm{k}$ per $\mathrm{F}$ respectively. The transient with the negative moderator coefficient exhibits a short term power increase as the average temperature is reduced. Note that there is an increase in the moderator margin to low pressure trip from the initial full power value.

Figure 9.22 shows the transient following a dropped rod worth $-2.5 \times 10^{-3}$ $8 \mathrm{k}$. In this case the prompt core power reduction is about 21 percent and the load cutback more nearly matches the core power. There is no steam release and equilibrium conditions are reached in four to six minutes.

\section{5 .3 CONCLUSIONS}

Protection for a dropped control rod is provided by the combination of an automatic turbine power cutback and blocking of automatic rod withdrawal. The magnitude of the power cutback is to be determined experimentally in conjunction with initial startup tests. The protection system will in all cases protect the core and will when at or near full power prevent a reactor trip without requiring manual control. 
(1) $\alpha_{\mathrm{w}}=-2.5 \times 10^{-4} \delta \mathrm{k} /{ }^{\circ} \mathrm{F}$

(2) $\alpha_{\mathrm{W}}=0$
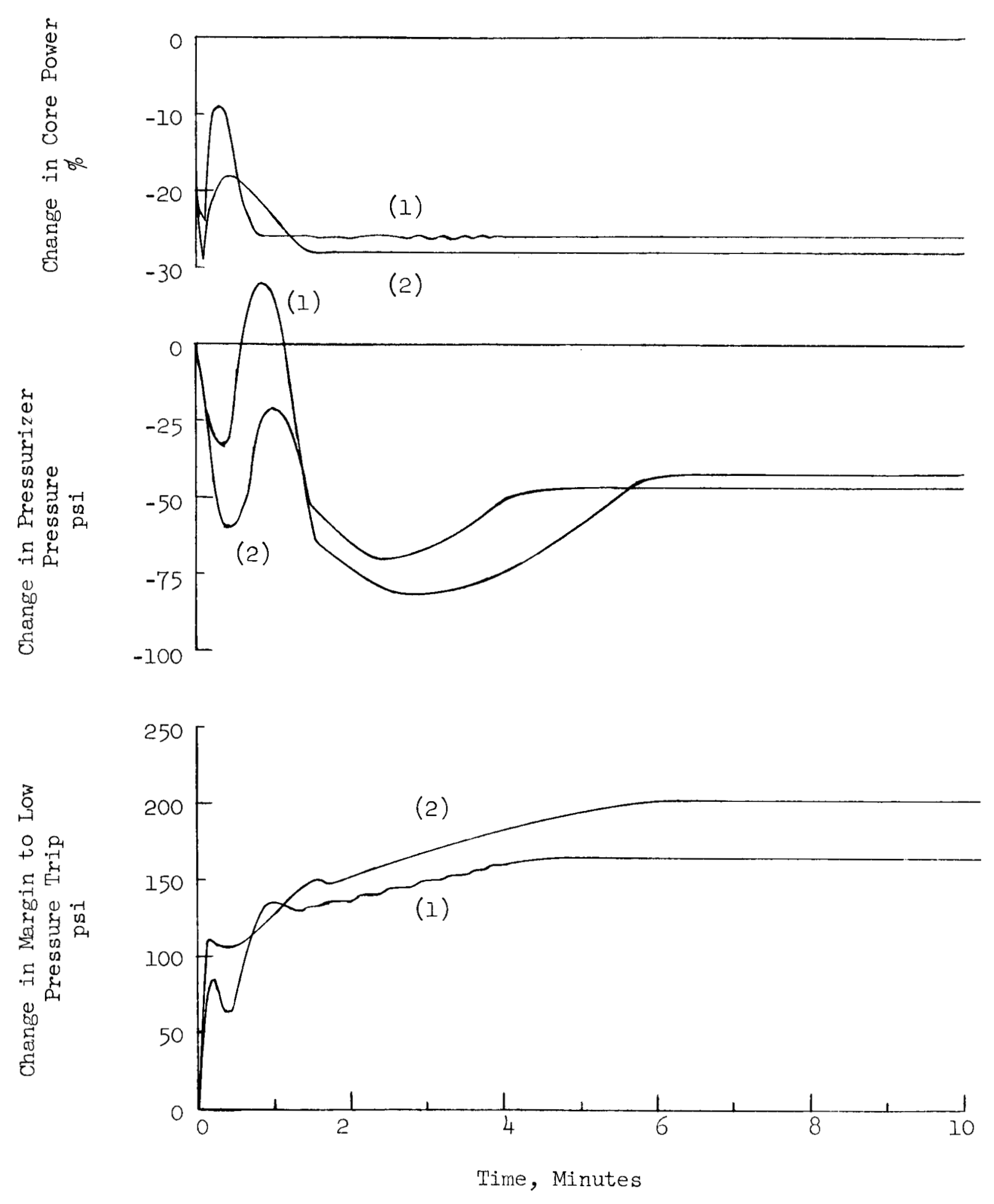

(1) $\alpha_{\mathrm{w}}=-2.5 \times 10^{-4} \delta \mathrm{k} /{ }^{\circ} \mathrm{F}$

(2) $\alpha_{\mathrm{w}}=0$
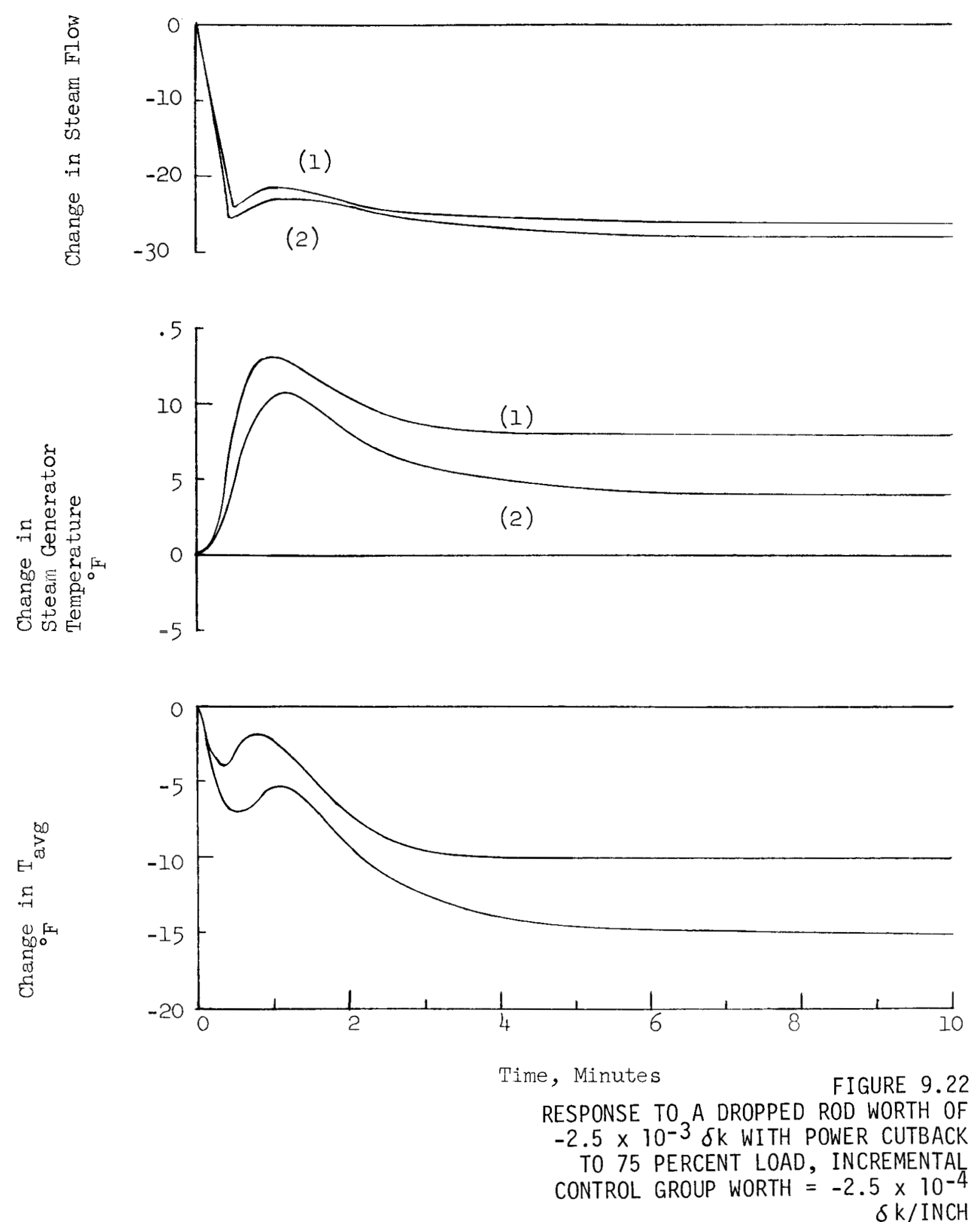


\subsection{CONTROL ROD EJECTION ACCIDENT}

\subsubsection{GENERAL}

The assumptions inherent for the postulation of a control rod ejection accident combine both the condition of a loss-of-coolant accident and the simultaneous insertion of reactivity associated with a given control rod. In order to assume a rapid ejection of the control rod from the core, a rupture of the control rod mechanisms must be postulated.

For this accident, it will be demonstrated that the effects are definitely localized, and that there is no resulting pressure surge that could further damage the Reactor Coolant System. Thus, it is assured that the ultimate consequences of the postulated accident would be less severe than an assumed double-ended rupture of the main coolant pipe.

In this postulated event, the control rod is ejected from the core in a very short time by full system pressure differential acting on the drive shaft. The resultant power excursion is limited by the Doppler reactivity effect of the increased fuel temperature, and terminated by reactor trip, actuated by high nuclear power signals. The amount of fuel damage that could result from such an accident is governed mainly by the peak power attained in the transient, which in turn depends on the worth of the ejected rod and the power distribution attained with the remaining control rod pattern.

In the following analysis, a series of assumed initial loading cases was studied. These included the rod ejection calculations reported in Paragraph 3.4, Reactor Nuclear Design. These calculations considered the power redistribution and rod worths of the highest worth rod removed from both the 29 and 17 rod control bank patterns. The nuclear analysis reviewed both the beginning-of-life and end-of-life configurations, because the control rod configuration changes with depletion of the core.

The results of these analyses indicated that there would be no fuel melting, and hence, no possibility of an excessive pressure transient in the Reactor Coolant System.

\subsubsection{FACILITY DESIGN ASPECTS}

A failure of a control rod travel housing sufficient to allow a control rod to be rapidly ejected from the core is not considered credible for the following reasons:

a) Each contro1 rod drive mechanism is completely assembled and shoptested at 6,300 psi.

b) The mechanisms will be individually hydrotested to 3,750 psig as they are installed on the reactor vessel head to the head adapters, and again checked during the hydrotest of the completed Reactor coolant System. 


\subsubsection{FACILITY DESIGN ASPECTS (Continued)}

c) Stress levels in the mechanism are not affected by system transients at power, or by thermal movement of the coolant loops. Because the mechanisms are restrained by cross-bracing at the top end, moments induced by the design earthquake can be accepted within the allowable primary working stress range specified by the ASME code, Section III, for Class A components. The design further assures that failure would not occur with an earthquake of twice the design intensity.

d) The pressure housing and the rod travel housing are each a single length of forged Type-304 stainless steel. This material provides excellent notch toughness at all temperatures that will be encountered, as demonstrated in Paragraph 3.2. The joints between the mechanism and the head adapter, and between the pressure and rod travel housings, are threaded with a seal weld canopy.

Programming of all the rods in the shutdown and control groups will be under strict administrative supervision to ensure proper sequencing and withdrawal in going to power. Al1 control rods have position indicating coils for indicating their position, thereby providing the means of surveillance over this operating limit. Separate and independent digital readout is also presented on the control board for the group or groups of rods used for control.

\subsubsection{ASSUMPTIONS}

\section{Genera1}

The nuclear analysis considered the control rod worth and power redistribution effects of various rods removed from both the 29 and 17 rod control group patterns. These analyses were performed at the beginning and end of core 1 ife. From the standpoint of this accident analysis, an ejected rod from the 45 rod pattern is trivial since the core would still be shut down by the remaining inserted rods.

\section{Beginning of Life, Zero Power}

The worth of the highest worth rod at beginning of life removed from the fully inserted 17 rod pattern was calculated as 0.4 percent $\Delta \mathrm{k} / \mathrm{k}$. The resultant power distribution for this case was only slightly higher than the full power design hot channel factors. Ejection of this rod would correspond essentially to the zero power condition, since this control bank is used alone in going to beginning of life power. It should be noted that this worth is substantially less than prompt critical. Thus the characteristic response would be a sudden jump of from two to two-and-one-half times the initial power, followed by a slow increase as delayed neutrons build up and as further reactivity changes are caused by fuel temperature and moderator density changes. Fuel temperatures approach the equilibrium values for the new power during the short time before reactor trip becomes effective. Thus the energy generated by the accident, for a given reactivity addition, is approximately proportional to the initial power level. Practically no energy input results from an accident at zero power. 


\title{
9.6 .3 ASSUMPTIONS (Continued)
}

\author{
Beginning of Life Conditions, Ful1 Power
}

For a short period at the beginning of the first core life, the moderator temperature and void coefficients will be slightly positive. During this time, the single seventeen rod control group provides enough control for a swing from zero-power to full-power. Ejection of the highest worth rod from the 29 rod control group pattern is again insignificant since there would be no return to criticality at beginning of life. Normally, the reactor would be at full power with the control banks in the upper portion of the core.

At power, the inserted worth of the entire control bank at beginning of life ranges from 0.2 to 0.8 percent $\Delta \mathrm{k} / \mathrm{k}$. The reactivity worth of a rod ejected from this pattern (approximately 0.04 to 0.06 percent $\Delta_{k} / k$ ), with the control bank operating in the upper portion of the core, is substantially less than the 0.4 percent $k / k$ value calculated for the zero-power case. The rapid insertion of the small amount of reactivity associated with a single rod ejected from this full-power pattern would have little effect on the maximum fuel temperature.

\section{End of Life Conditions, Zero Power}

Later in life, as boron is removed from the coolant to compensate for burnup, the moderator temperature and void reactivity coefficients become increasingly negative. Because of this, greater control rod worth is required to shut down from full power than at beginning-of-life conditions, to make up for the moderator reactivity gained by cooling down from full-power temperature to zero-power temperature. In the extreme, at the end of life, the moderator temperature coefficient will be approximately $-2 \times 10^{-1} \Delta \mathrm{k} / \mathrm{k}-\mathrm{F}$; the hot zero-power condition would have the seventeen-rod bank fully inserted; and the twelve-rod bank would be almost fully inserted at zero power. Ejection of the highest worth control rod from this hot zero-power condition $\left(10^{-6} \times 1,347 \mathrm{Mwt}\right)$ would result in a heat flux hot channel factor of almost 10 , and a calculated maximum ejected rod reactivity of 0.8 percent $\Delta \mathrm{k} / \mathrm{k}$.

As an extremely pessimistic approach, this accident at zero power was studied using a zero-moderator coefficient, and a low estimate for the endof-life delayed neutron fraction, 0.0050 . A fuel temperature reactivity weighting factor of 2.0 ; a fuel-to-clad gap heat transfer coefficient of $2,000 \mathrm{Btu} / \mathrm{hr}-\mathrm{ft}^{2}-\mathrm{F}$; a prompt neutron lifetime of 13 microseconds; rod ejection time of 0.1 second; time to start effective reactivity insertion following reactor trip of 1.0 second; and a trip rod reactivity of 1.8 percent were used. The weighting factor is the ratio of the fuel reactivity feedback to the reactivity, calculated from the change in core average fuel temperature.

\section{End of Life, Full Power}

At end of life, both control banks must be utilized to go to full power. With the established withdrawal sequence, as the control banks are successively withdrawn, the worth of an ejected rod is reduced and also the ejected rod hot channel factors are improved. At full power, towards end 


\subsubsection{ASSUMPTIONS (Continued)}

of life, the rods will be operated substantially in the upper portion of the core with very little inserted worth. The maximum ejected rod reactivity insertion calculated for full power is 0.04 percent $\Delta \mathrm{k} / \mathrm{k}$. The total hot channel factor is not significantly greater than the design value. Thus the rapid insertion of this amount of reactivity would, as in previously described case, cause only a slight rise in fuel temperature.

\subsubsection{METHODS OF ANALYSIS}

\section{Reactor Kinetics}

The nuclear power transients were calculated using the CHIC-KIN code, developed by the Bettis Atomic Power Laboratory for similar analyses (J. A. Redfield, "CHIC-KIN -- A FORTRAN Program for Intermediate and Fast Transients in a vater Moderated Reactor", WAPD-TM-479, January 1965). This code solves the point reactor kinetics equations, with feedback from an axially-segmented fuel element. Both local and bulk boiling voids are accounted for, providing a conservative extreme for the positive void reactivity case. For this study, the fuel rod was divided into eight radial increments, with a ninth increment for the clad. A fuel-to-clad gap heat transfer coefficient of $2,000 \mathrm{Btu} / \mathrm{hr}-\mathrm{ft} \mathrm{t}^{2}-\mathrm{F}$ was used as a conservatively high value. The calculated fuel temperature reactivity was based on the core average transient fuel temperature rise, with a fuel temperature reactivity weighting factor of 2.0 for the 0.8 percent insertion case.

In order to obtain an extreme positive moderator reactivity feedback for the beginning-of-life case only, the entire core power increase was assumed to be concentrated in one-fourth of the core. This is consistent with iterative physics calculations that indicated this pattern of energy input to the coolant would give the maximum positive reactivity.

The physics calculations consisted of performing two-dimensional analyses of the core, with different amounts of core assigned lower water density than is normally the case. These calculations were performed for both the unrodded core, the core containing one control bank with its highest worth rod ejected entirely out of the core. Analyses were also carried out for two different values of water density lower than the normal full-power operating value.

All studies indicated that, for a core divided into a normal full-power outer region and a center region at some lower water density, the size of this center region, corresponding to the maximum reactivity insertion, is 25 percent of the total core. In order to obtain the value of the maximum reactivity that could be inserted in this fashion, the same kind of twodimensional calculations were performed for different values of water density in the center quarter of the core.

The results are presented in Figure 9.23 for the unrodded core, and for the core containing one control bank less its highest worth rod where the multiplication factor is plotted as a function of water density in the center quarter of the core. Results are given for a range of densities from 


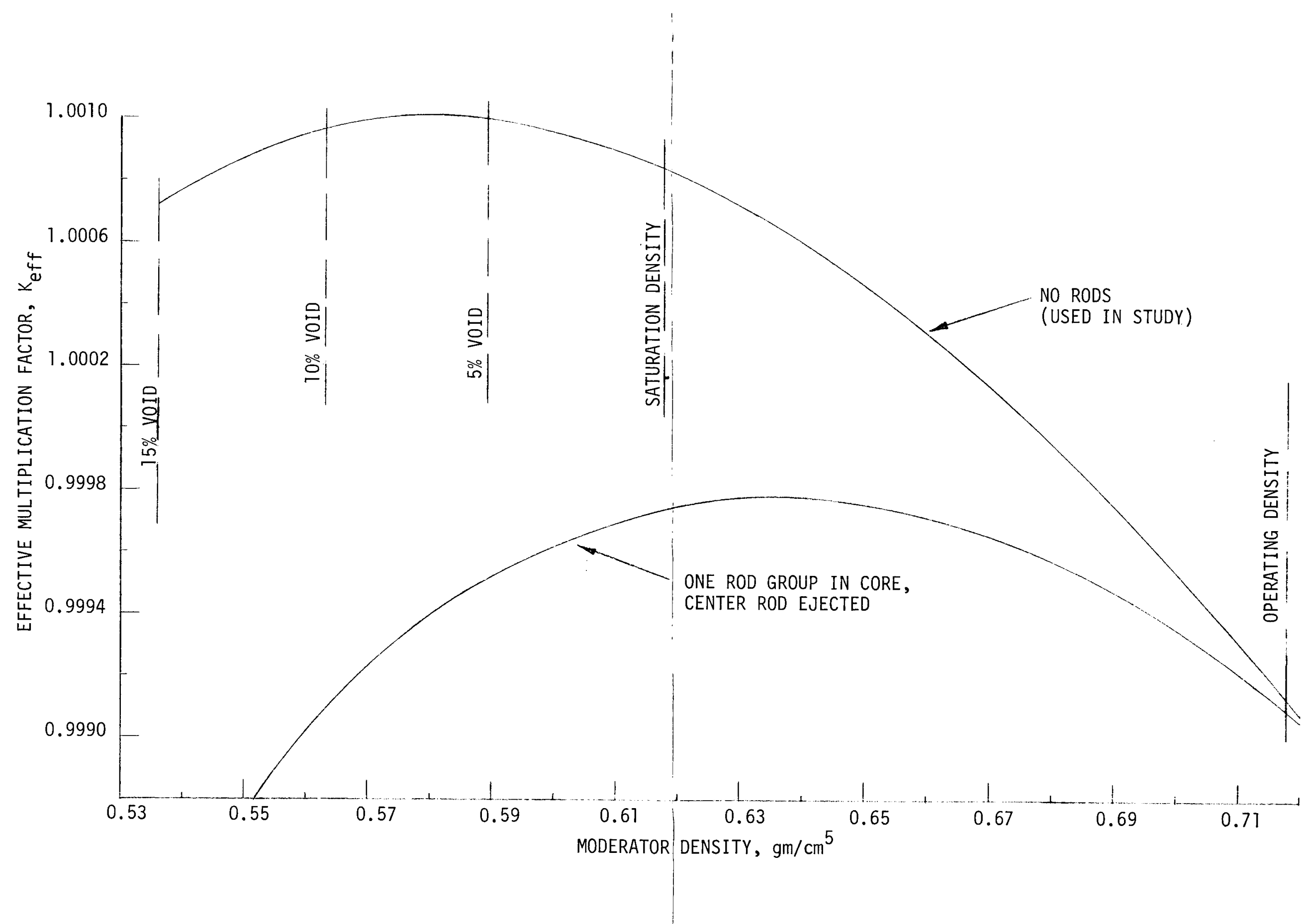

FIGURE 9.23

EFFECTIVE MULTIPLICATION FACTOR VERSUS MODERATOR DENSITY FOR DENSITY CHANGES IN 25 PERCENT OF THE CORE 2500 PPM BORON 


\subsubsection{METHODS OF ANALYSIS (Continued)}

normal full power to 15 percent void content in this center region. It can be seen that the greatest moderator density reactivity insertion is 0.19 percent in the unrodded core, and that the value in the case of the ejected rod is only 0.07 percent (i.e., the maximum Keff minus the Keff at operating density. From the curve, for the unrodded core, the figures are 1.00 $1.00101-.99914=.00187=0.19$ percent. For the curve with one contro1 bank in the core, the figures are $.99978-.99909=.00069=0.07$ percent.) In the rod ejection transient calculations, the moderator feedback curve for the unrodded core was used, as a conservative extreme.

The resultant calculated energy storage in the fuel center was then normalized to the calculated worst hot-channel factor to obtain the maximum core fuel temperature. (Because heat continues to be added for about a second after the initial power burst, the maximum fuel temperature reached for all cases studied is in the fuel center.)

\section{Fission Product Release}

Assumptions governing the determination of fission product released to the coolant, as used in this analysis, are as follows:

a) Cladding failure in any fuel rod releases the equilibrium fission products in the pellet-cladding gap. Cladding failure is assumed to accompany DNB.

b) A "burst" of fission products, conservatively estimated to be equal to that produced in 180 hours of diffusion from the fuel at normal operating temperature, occurs due to transient-induced reorientation of the surface layer of uranium oxide.

c) Diffusion in the pellets whose average temperature exceeds $2,900 \mathrm{~F}$, but where center melting is not attained, occurs at an accelerated rate, $10^{5}$ times normal. This rate, which is actually characteristic of the diffusion at temperature very near the melting point (i.e. $5,000 \mathrm{~F}$ ), is assumed to persist for the duration of the fuel temperature transient.

d) In computing the fission product inventory of the fraction of the core subject to each type of release, the steady state power distribution is compounded to give the maximum possible fission product release.

\section{System Pressure Surge}

Calculations were also performed to determine whether the pressure surge, resulting from the rapid increase in heat transfer through the fuel cladding, might cause a coolant volume increase sufficient to exceed the allowable system pressure. A digital computer code was used that accounted for steam void formation in the core and mixing in the hot leg; pressure drop in the pressurizer surge line; and load rejection due to turbine trip caused by the overpower reactor trip. To achieve even more conservative 


\subsubsection{METHODS OF ANALYSIS (Continued)}

calculations, no condensation of steam by the water stored in the reactor vessel outlet plenum was assumed. For this calculation, the release of coolant through the head penetration for the ejected control rod was neglected.

The pressure increase is a function of the heat transferred to the water in the coolant; the initial coolant temperature; the initial heat transferred to the steam generator; and the initial pressurizer level. For a given increase in reactor heat flux, the highest pressure would result if the plant were initially at full. power, because at that time all these parameters would be at a maximum. At full power, however, the possible ejected rod worth, and thus heat flux increase, is much smaller than at low power.

\subsubsection{RESULTS}

\section{Power Increases and Fuel Temperatures}

At beginning of life, with the calculated worth of rods from zero-power and full-power configurations, there was little increase in core power for the zero-power case and only a slight increase in fuel temperature for the full-. power case.

At full power, toward the end of core life, the control rods will be operated substantially in the upper portion of the core with very little inserted worth. The maximum ejected rod worth calculated for full power was 0.04 percent. The core power distribution was only slightly higher than the design value. The rapid insertion of this amount of reactivity would cause only a slight rise of approximately $100 \mathrm{~F}$ in fuel temperature.

Figure 9.24 demonstrates the nuclear power transient resulting from an ejected rod at the end of life, with the control rod configuration at zero power, but with zero moderator, void, and temperature reactivity coefficients. A reactivity insertion of 0.8 percent $\Delta \mathrm{k} / \mathrm{k}$ and a resulting tota 1 hot channel factor of 10.0 were assumed. Even under these severe conditions, the resulting maximum hot-spot fuel center temperature only reached 4,060 F, well below the fuel melting temperature. The temperature of the outer part of the pellet did not get as hot, and was cooling as the center reached its peak. Even in this isolated case, where was no danger of molten fuel being expelled into the coolant. The fraction of rods in DNB was calculated to be 11 percent, while the fraction of fuel pellets with $\mathrm{T}_{\text {avg }} \geq 2,900 \mathrm{~F}$ was on1y 1.0 percent.

\section{Fission Product Release}

The fission product release was conservatively calculated to be equal to or less than one percent of that equivalent to a complete core meltdown.

Pressure Surge

The core fuel-to-water heat flux calculated for: the zero power conditions is shown in Figure 9.25. The maximum calculated pressure resulting from 


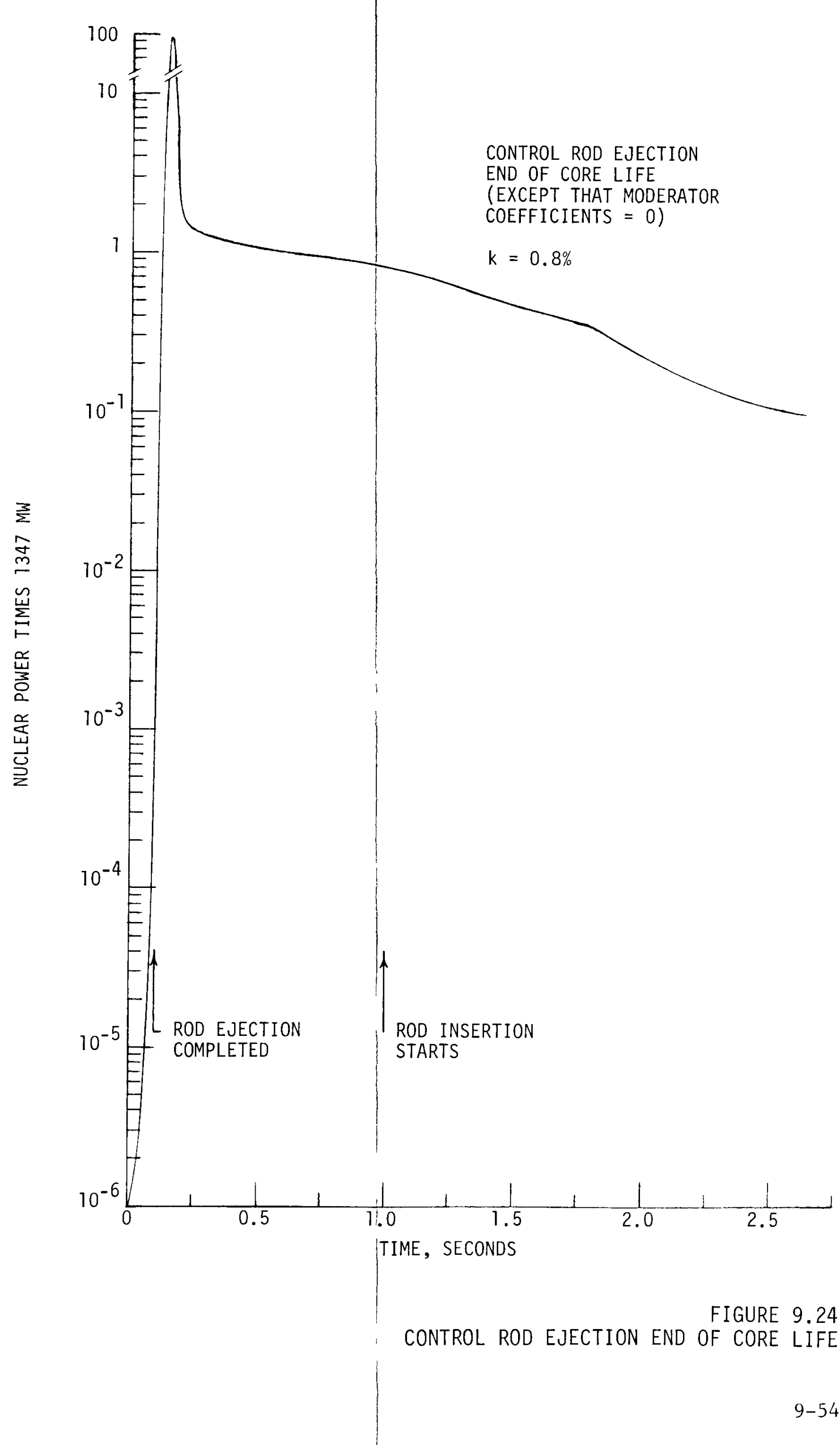



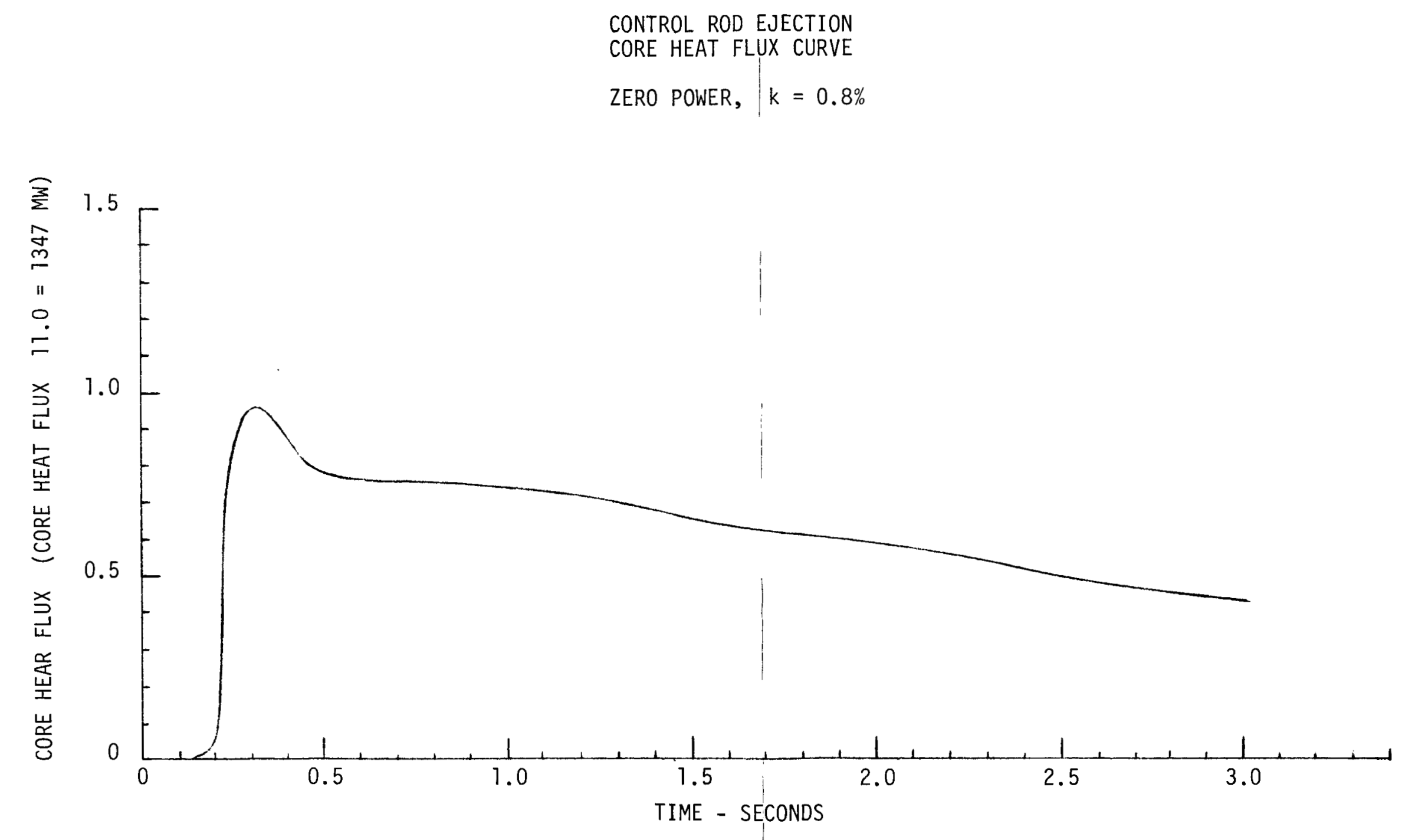

FIGURE 9.25

CONTROL ROD EJECTION CORE HEAT FLUX 


\subsubsection{RESULTS (Continued)}

this transient, only 2,316 psia, would occur 9.7 seconds after the accident.

\section{6 .6 CONCLUSIONS}

The rod ejection analysis was reviewed for the control rod configurations specified for zero and full power conditions at the beginning and end of life. The results of this analysis indicated that there was no melting of the fuel, even for the conservative assumptions postulated. For the fullpower cases, where the center temperature was initially high, the resulting temperature increase was sma11. The operation of the plant precludes the insertion at power of any rod of substantial worth. The zero power case, at end of life, resulted in the highest increase in the center temperature of the fuel; however, this was still substantially below the melting temperature.

Thus there is no chance of any rapid expulsion of finely dispersed molten fuel with the coolant and further propagation of the accident. Experimental results show that gross fuel melting, preferentially located in the vicinity of the clad surface, must occur before fragmentation of the fuel pellet clad system could occur. Since this analysis indicated that there was a substantial margin to any gross fuel pellet melting, it was concluded that there could not be a substantial pressure surge in the Reactor Coolant System. The maximum pressure due to the increased heat flux was determined in all cases to be equal to or less than 2,316 psia, well below the design pressure of 2,500 psia.

The maximum size break for this postulated accident is on the order of four inches. The total break and resultant rate of energy released to the containment sphere was postulated to be much less severe than those cases studied for the hypothetical accident described in Section 14.

The amount of fission products released to the containment also resulted in an amount well below that determined for the hypothetical accident described in Section 14, even with the conservative assumption that there was no retention of the fission products in the coolant during the relatively slow blowdown of the coolant. 


\section{SECTION 10 \\ MECHANICAL MALFUNCTION ANALYSES}

\subsection{LOSS OF COOLANT}

\section{1 .1 GENERAL}

Loss of coolant is a condition of accidental discharge of fluids from the reactor coolant system, which is a breach in the third of the barriers to fission product transport. Reactor protection involves tripping the reactor and supplementing the supply of coolant to prevent or limit damage to the fuel cladding. Public protection is ensured by the containment sphere.

The following types of coolant loss are treated in this evaluation:

a. Rupture of any line or nozzle connecting to the reactor coolant system.

b. Improper opening of a valve, or of valves in series, allowing uncontrolled discharge from the high-pressure reactor coolant system to a low-pressure system.

c. Material failure in a pipe or component of the reactor coolant system, resulting in an opening with an effective size not greater than the largest in categories a and $b$ above.

A special case of loss of coolant, treated in Paragraph 10.5, is the rupture of a steam generator tube. For this failure, one must consider the possibility of transport through the steam system of some of the activity present in the reactor coolant. The analysis and description of consequence-limiting features must take the steam system operation into account and is therefore considered separately. Similarly, a severance of a control rod drive pressure housing with resultant ejection of the rod cluster from the core, while constituting a loss-of-coolant fault, is treated separately in Paragraph 9.6 because of the special conditions introduced by the reactivity insertion.

\subsubsection{FACILITY DESIGN ASPECTS}

\section{Preventive Features}

The importance of the loss-of-coolant class of mechanical failures merits consideration of the many features designed to prevent the occurrence of a loss of coolant. These features provide assurance of the integrity of the reactor coolant barrier by:

- Conformance to high standards of materials and construction

- Control of mechanical and pressure loads 


\subsubsection{FACILITY DESIGN ASPECTS (Continued)}

- Control of the environment of material exposure

- Surveillance

The manner in which each of these areas affects the integrity of the reactor coolant system is amplified in the following paragraphs.

a) Material and Construction Standards - A summary of the applicable codes and standards, including special nuclear case rulings, under which the components of the reactor coolant system and the reactor vessel itself are designed is contained in Paragraph 3.5, Reactor Coolant System. Within the rules of the above codes, configurations and thicknesses of pressurebearing members are established by specified calculations to provide acceptable margins of safety against overstress during design loading conditions.

The applicable design code specifies acceptable materials, fabrication procedures, and test requirements which give assurance that the properties inherent in the application of the code rules are obtained. Paragraph 3.5 also lists the material specifications for all significant pressure-bearing components of the reactor coolant system, showing the selection of code materials.

In addition, provisions of the ASA B31.1 piping code are applied to loop piping in the reactor coolant system to limit the allowable stress range for cycle loadings. Otherwise, the basis for design against fatigue-type failures is that recommended in the "Tentative Design Basis for Reactor Pressure Vessels and Directly Associated Components" (PB 151987), or a modification of this basis utilizing the latest appropriate data from Section III, ASME Boiler and Pressure Vesse1 Code, "Nuclear Vessels." This basis incorporates a low-cycle fatigue design method which assures that allowable usage of the fatigue life of the material is not exceeded, even in the unlikely event that the full number of cycles assumed for design are actually imposed on the plant.

A piping flexibility analysis was performed for the reactor coolant piping to show that the maximum stress range induced in the pipe and fittings by the constraint of thermal distortions are within the limits of the piping code. A similar analysis was performed with seismic and dead loads imposed on the piping. Each of these analyses confirmed that the location of hangers and component supports, and the dimensional allowances for unrestrained deflection are adequate.

In the structural analysis of other reactor coolant system components, piping end forces, dead loads and seismic loads are applied to show that the design of supports and stops is adequate to code allowable stress limits. The criteria applied are highly conservative in that seismic forces are considered as primary loads, i.e., continuous rather than transient as is customary in seismic design of structures. Seismic forces are determined by the method outlined in Paragraph 11.2 for components essential to the nuclear safety of the plant. 


\subsubsection{FACILITY DESIGN ASPECTS (Continued)}

b) Control of Pressure, Thermal and Mechanica1 Loads - Pressure loads are limited in accordance with the applicable design codes by the provision of self-actuated safety valves of specified characteristics. These valves are sized to control the pressure surge to the code limit of 110 percent of the maximum allowable working pressure of 2,485 psig in the improbable condition where 100 percent load rejection occurs without reactor trip or feedwater addition. All operational transients and load rejections are less restrictive than this case to the design of the pressure relieving devices to the reactor coolant system.

The largest pressure surges incurred under design load transients are controlled below 2,185 psig at the pressurizer by operation of either of two power relief valves. Smaller transients are controlled within this limit by pressurizer spray.

The possibility of reactor faults giving rise to pressure surges in excess of power relief valve setting is discussed in Sections 9 and 10 . In general, all credible reactor faults are terminated by the reactor protection system before an overpressure condition arises, even if one failure is assumed to occur in the protection system itself.

Thermally induced local stresses are limited by the provision of thermal sleeves at all inlet connections where introduction of cold fluid may occur a significant number of times during the life of the plant. The connections so protected are the surge line and spray connections at the pressurizer, and the reactor coolant loop-inlet connections of the charging line and residual heat removal loop. Temperature changes in the reactor coolant system which occur as a consequence of load changes, reactor trips, and planned heatup and cooldown are less severe and the expected cycles far less numerous than the values assumed for the purpose of fatigue analysis. Control of these transients is inherent in the reactor control system response characteristics, except for the heatup and cooldown cycle which is manually controlled. Heatup and cooldown rates will be adjusted to ensure that thermal stress in the reactor vessel shell conforms to limits imposed by the temperature and radiation history of the vessel.

Mechanical loads on the reactor coolant system piping and components are controlled by proper design of supports, hangers, and limit stops to accommodate thermal expansion and to resist seismic and dead loads. The restraining forces exerted by these structures are considered in the mechanical design analysis of the respective components.

c) Control of the Environment - Environmental control is the principal means of protection against corrosion-type failures. The pressure bearing materials exposed to the reactor coolant environment, austenitic stainless steel and Inconel, are highly resistant to general surface corrosion in water because they both form tightly adhering oxide films which inhibit mass transfer to and from the base metal. The rate of attack by other mechanisms, i.e. galvanic effects and stress corrosion cracking, is sensitive to the presence of chlorides and oxygen in solution. Control of reactor coolant chemistry, limits the concentration of chlorides and 


\subsubsection{FACILITY DESIGN ASPECTS (Continued)}

dissolved oxygen. These precautions are taken to assure a margin of about 10 to one below the minimum concentrations of these agents which have been known to exist in cases of stress corrosion attack.

The corrosion rates which have been measured under the conditions of reactor coolant system operation, including average concentrations of boric acid, are such that an integrated material loss of the order of two mils for stainless steel and four mils for Inconel would be expected in the life of the plant. Corrosion at this rate will have no effect on the integrity of the reactor coolant system.

d) Surveillance - Surveillance of the reactor coolant system to assure continued integrity will be carried out through the following programs:

1. Continuous monitoring of reactor and coolant system operating variables; corrective action can be taken according to planned procedures even before operating limits are reached.

2. Periodic sampling of reactor coolant assures continuity of the chemical environment which precludes corrosive deterioration of the system.

3. Periodic removal and testing of reactor vessel material specimens exposed to the environment and incident flux levels seen by the vessel itself at the core midplane, to verify the adequacy of provisions for the anticipated shift in the nil ductility transition temperature.

4. Removal and inspection at refueling intervals of the reactor vessel head studs; visual and dye penetrant examinations to check the continued integrity of these items.

Protection of Reactor Core

The occurrence of a loss of coolant, unless of a very small magnitude, will bring into operation one or more means of supplementing the supply of reactor coolant to prevent or limit possible damage to the fuel. The means of supplying this protection will depend on the magnitude of the rate of coolant loss. In the order of increasing loss rate, these actions are:

a) For small breaks, pressurizer leve 1 control adjusts the net charging-letdown rates to maintain a minimum level in the pressurizer. Depletion of coolant in the volume control tank is compensated by automatic makeup control at reactor coolant boron concentration (refer to the Chemical and Volume Control System discussion in Paragraph 3.6).

b) For larger breaks, or in case of malfunctions of automatic makeup, an emergency low-level signal from the volume control tank-level instrumentation enables the charging pumps to take suction from the refueling water storage tank instead of the volume control tank. Valving to accomplish this transfer is duplicated for reliability. 


\subsubsection{FACILITY DESIGN ASPECTS (Continued)}

c) For still larger breaks, i.e., those for which the discharge rate exceeds the delivery of the charging pumps for a prolonged period of time, the level in the pressurizer will continue to decrease, eventually exceeding the low-level trip point for safety injection. A change in the pressurizer steam volume, will result in an accompanying decrease in system pressure. The coincidence of low-pressurizer pressure with low pressurizer level, requiring signals from two out of three channels, will actuate the safety injection system, as described in Paragraph 5.1. This actuation is fully automatic and consists of:

1. Tripping the reactor.

2. Isolation of the turbine cycle feedwater-condensate system from the feedwater pumps by valve action.

3. Starting the two safety injection pumps, both feedwater pumps (if not already running), and the standby charging pump.

4. Stopping the four condensate pumps and two heater drain pumps.

5. Aligning charging pump suction with the refueling water storage tank (if not already accomplished).

5. Opening the safety injection isolation valves at the reactor coolant loops.

7. Opening the safety injection isolation valves at the feedwater pump discharge (only after the condensate isolation valves at the feedwater pump suction are verified closed).

Actuation relays and related circuitry to accomplish these steps are fully duplicated for reliability. Completion of safety injection actuation results in pressurization of the injection header and an open path for delivery of borated water from the refueling water storage tank through two independent safety injection trains and three independent injection lines, one to each reactor coolant loop. In addition, both charging pumps are running, and delivering borated refueling water to the charging line.

The flow rate of refueling water delivered to the reactor coolant system by the combination of pumps just described will vary with the pressure head against which they are delivering. The delivery characteristics of the system, shown in Figure 10.1, are based on pump manufacturers' performance curves, with allowances for the calculated flow resistances of the various delivery paths. Five cases are shown, representing full system operability and four partial operability modes representing single failure conditions. These cases are identified in the following manner: 
CONDITIONS

$\frac{\text { CASE NO. }}{\text { I II III IV V }}$

No. of S. I. Trains Functioning

No. of S. I. Lines Injecting

No. of S. I. Lines Spilling to Containment

No. of S. I. Lines Blocked

* Flow limited to $500 \mathrm{gpm}$ plus the higher of the two injecting lines.

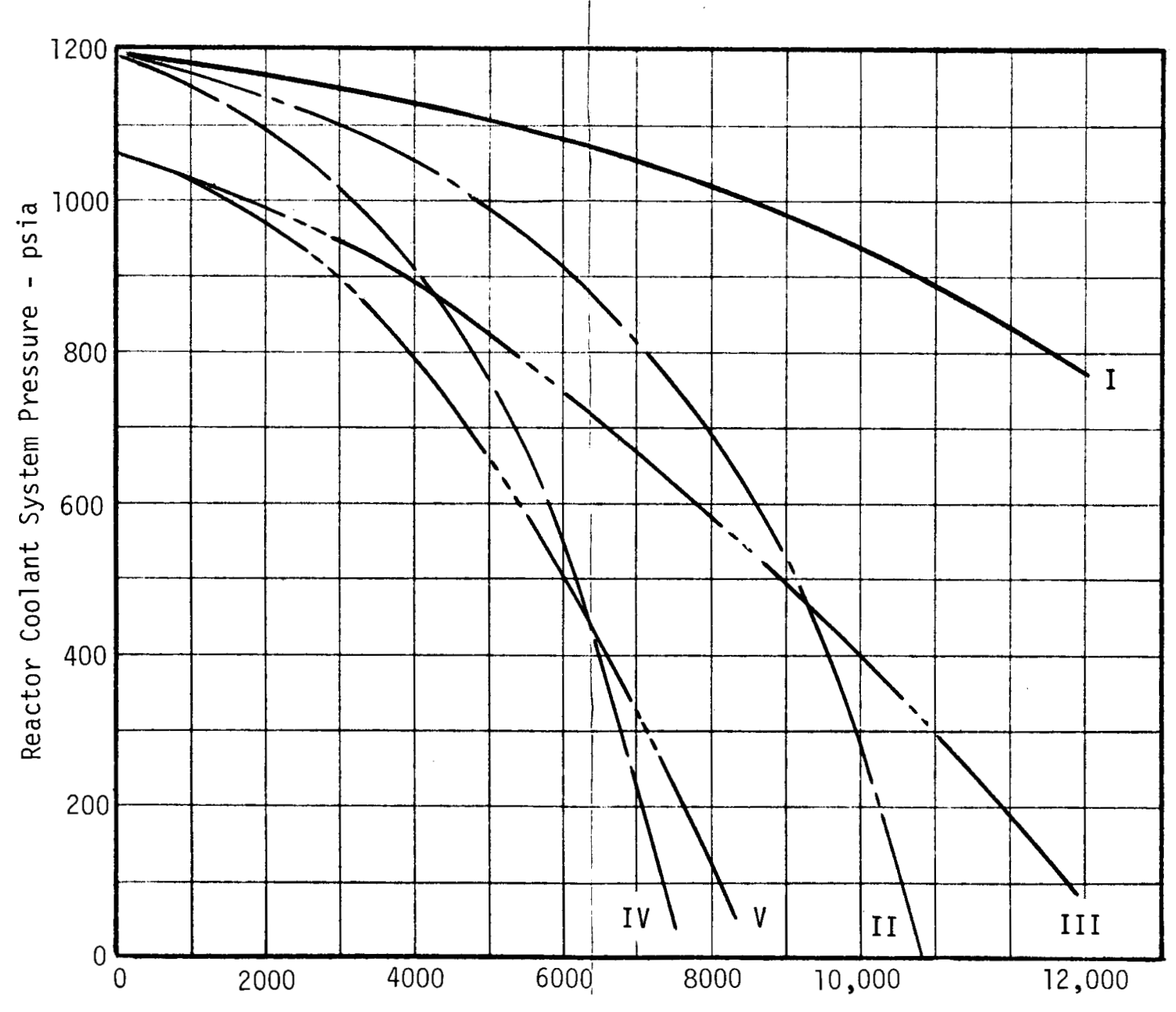

Injection Rate - Gal/Min

FIGURE 10.1

SAFETY INJECTION DELIVERY

CHARACTERISTICS 


\subsubsection{FACILITY DESIGN ASPECTS (Continued)}

Case I - Both safety injection trains operating; delivery through three injection lines.

Case II - One safety injection train operating; delivery through two injection lines, with third line isolated.

Case III - Two safety injection trains operating; delivery through two injection lines, with third line spilling to the containment.

Case IV - One safety injection train operating; delivery through two injection lines, with third line spilling.

Case V - Two safety injection trains operating; one injection line operating; one injection line isolated; one injection line spilling to the containment.

Note that case I represents full system capability. Case II allows for the failure of one train to operate, whether by malfunction of a pump, valve or electrical circuit; it assumes one injection line isolated, denoting either (1) failure to open one injection line isolation valve or (2) operation of the flow comparator to limit the loss through a broken safety injection line. Case III assumes failure of the flow comparator, allowing full flow through a broken injection line to be discharged to the containment.

Normal functioning of the flow comparator would isolate the broken injection line unless the flow in that line were within $500 \mathrm{gpm}$ of the other two lines. Case IV assumes that the flow through the broken line is within the range where the flow comparator is non-sensitive (less than $500 \mathrm{gpm}$ ); hence, no isolation signal would be expected. The failure assumed in this case is the loss of one pumping train. Case $V$ represents another condition for a loss of coolant involving isolation valve failing in the closed position blocking delivery through one of the undamaged lines. The flow comparator is prevented from closing the valve in the spilling line in this case, but pumping through both trains is assumed. Credit for charging flow has not been assumed in the analysis for all cases.

In the general case, injection maintains sufficient water in the reactor to dissipate the residual heat of the core, either by boiling (with steam discharging through the break) by heat transfer to the steam generator tubes, or by the net enthalpy increase of injection water spilling from the break. Reactor Coolant System pressure decreases as the temperature is gradually reduced. Operation of the recirculation loop of the Safety Injection system is started by manual action to return borated water from the Containment Sphere sump to the Reactor Coolant system by way of the recirculation heat exchanger.

Loss-of-coolant analysis is performed to show conformance to the following criteria:

a. Under no condition would there be fission products released from the fuel rods as a result of a Reactor Coolant System rupture of credible size (i.e., not exceeding the effective size of the teninch severed comnecting line). 


\subsubsection{FACILITY DESIGN ASPECTS (Continued)}

b. Protection against larger size ruptures shall be effective in substantially limiting the fission product release.

c. The reliability of the protection described in a and $b$ above sha11 be such that the criteria are met even in the event of a failure of any single component of the protection system to respond actively in the prescribed manner.

\subsubsection{ASSUMPTIONS AND METHOD OF ANALYSIS}

The analysis of the loss-of-coolant blowdown transient is performed with the digital code LOCO, using an IBM-7094 computer. The mathematical model used by this code is summarized in Paragraph 10.1.6.

\section{Simplifying Assumptions and Initial Conditions}

a. The Reactor has been operating at full-rated power for an infinite time prior to the break.

b. The break occurs instantaneously, but nuclear heat generation and heat removal by the steam generators continue at full-rated power until the requisite conditions of low pressurizer pressure and low pressurizer level are reached, and until an additional instrument response and reactor trip time of 1.5 seconds has elapsed. After reactor trip, heat generation follows the calculated residual power relation, described in Paragraph 3.5 .

c. Heat removal by the steam generator is assumed until the liquidvapor interface falls below the level of the reactor nozzles. An expression for the difference between residual heat and steam generator heat-removal rate is used in the LOCO energy balance equations.

d. Sensible heat released by cooling the core and one-third of the vessel and thermal shield, assuming the temperature of these masses to fall at the same rate as the coolant temperature, is transferred to the coolant during blowdown.

e. Expulsion of the coolant through the break is governed by the mechanisms of subcooled flow until the calculated system pressure falls to saturation at the mass-average temperature.

f. After saturation is reached, two-phase flow through the break occurs with no slip. System pressure remains at saturation level, with flow limited by sonic choking as long as the pressure ratio across the break exceeds the critical value for the mixture.

g. When the steam-water interface falls below the level of the break, pure steam flow is assumed if the calculated entrainment is less than one percent. The LOCO code calculates the point in the transient at which this steam flow is obtained. 


\subsubsection{ASSUMPTIONS AND METHOD OF ANALYSIS (Continued)}

h. Following initiation of the safety injection signal and a time delay for repositioning valves, injection flow is added to the system at a rate determined by the applicable delivery curve for the system pressure at that instant.

In those cases where partial or complete uncovering of the fuel rods occurs, the temperature transient of the core is calculated using the method described in Paragraph 12.1. This analysis consists of a heat balance on the fuel rod, with heat input from both residual heat and metal-water reaction at the cladding surface, internal heat transfer among three pellet regions and a cladding region, and no heat loss from the cladding surface while the core is exposed to a steam atmosphere.

The pellet and cladding temperatures in each of ten core regions, representing various fractions of the peak steady-state power density, are evaluated by this method as functions of time after break. The maximum temperature of the cladding in each core region is compared with a calculated failure temperature to determine the extent of clad rupture, if any, resulting from the accident.

The aggregate steady state thermal power of those core regions determined to undergo clad bursting is used to calculate the release of volatile fission products from the pellet-cladding gap, as detailed in Paragraph 13.1.

Similarly, the maximum temperature attained by the fuel pellet in each of the core regions is determined. The thermal power of each core region, and its maximum fuel pellet temperature is used to calculate the fission product evolution from the $\mathrm{UO}_{2}$, following the method described in Paragraph 13.1.

\subsubsection{RESULT'S OF BLOWDOWN ANALYSIS}

Figures 10.2 through 10.8 show the calculated pressure and volume histories for a complete range of break sizes and for the various safety injection performance cases described earlier.

Break sizes corresponding to six nominal connecting pipe diameters (singleended rupture of 1-, 2-, 4-, 6-, 8-, and 10-inch lines at the point of connection) and the hypothetical double-ended rupture of the main reactor coolant loop were considered. Certain combinations of break size and assumed safety injection performance are not compatible and were excluded from the analysis for reasons listed below.

Case I - Hypothetical 27-1/2 inch break size

Case II - Hypothetica1 $27-1 / 2$ inch break size
Severance of a main loop could cause the associated injection line to discharge directly to the containment through the break.

Flow comparator would not be sensitive to flow differential; third injection line would not be isolated but would instead lose its flow via the loop break (covered by case IV). 

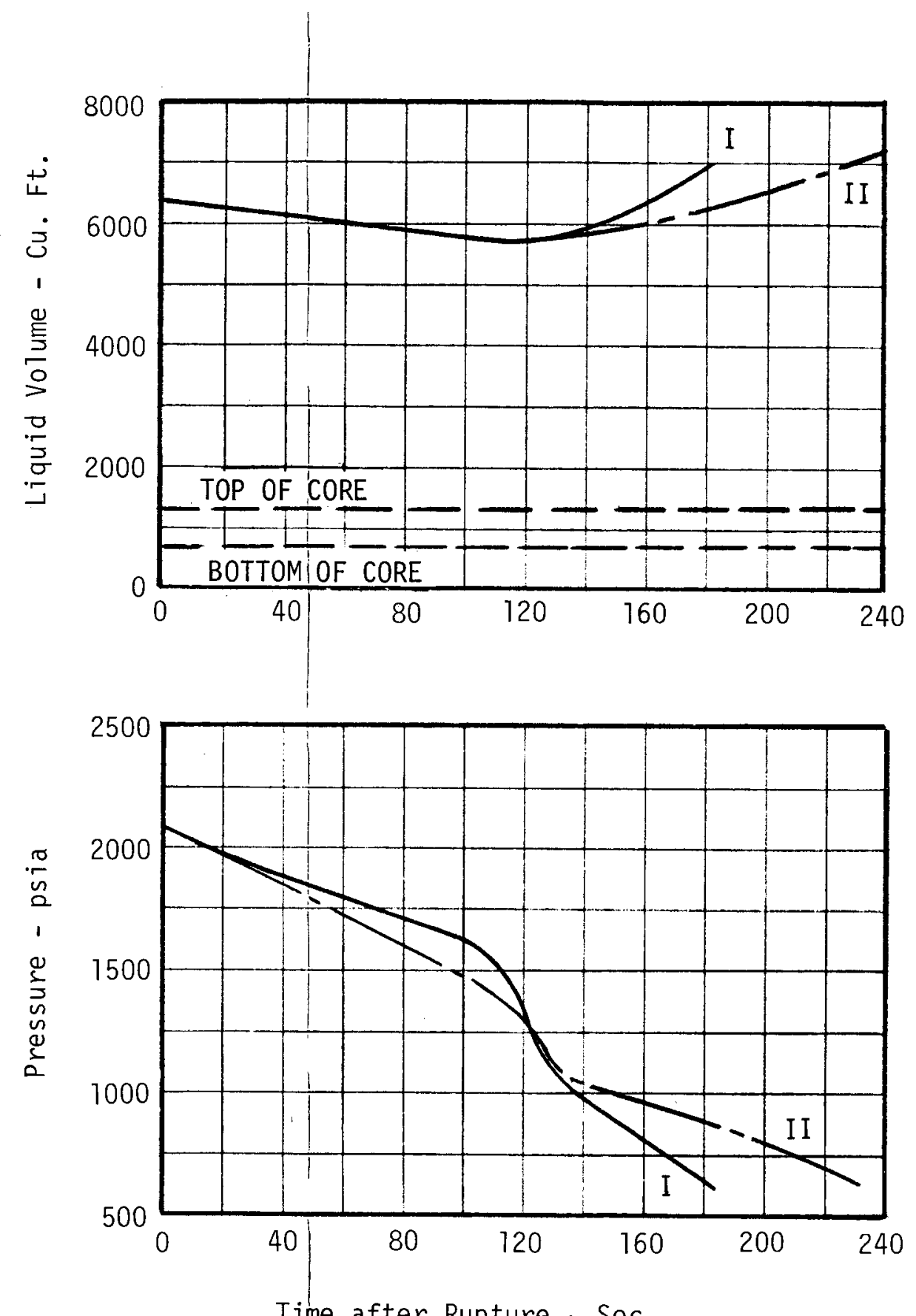

Time after Rupture - Sec.

FIGURE 10.2

LOSS OF COOLANT - ONE-INCH (NOMINAL)

PIPE BREAK 

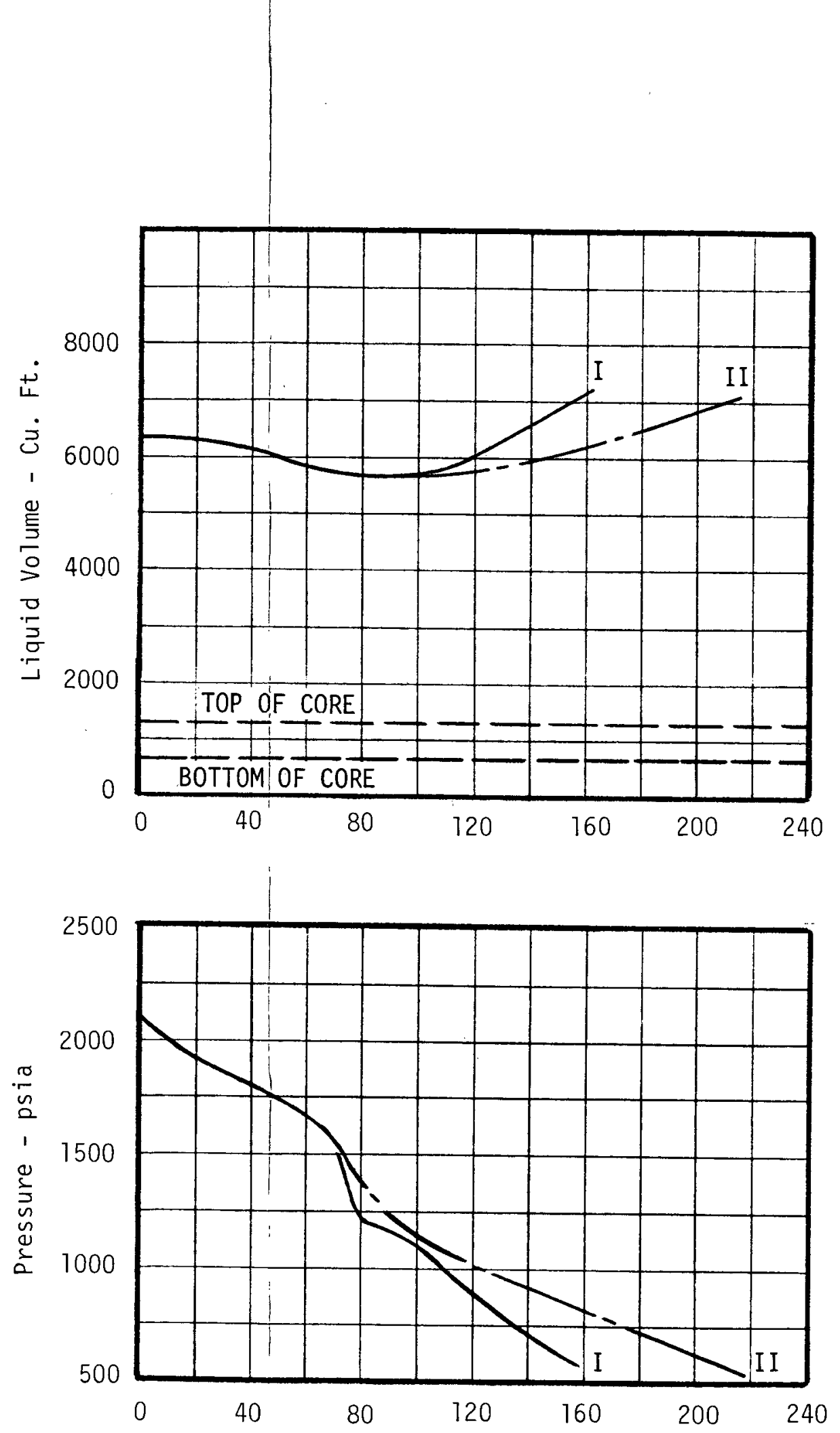

Time After Rupture, Sec.

FIGURE 10.3

LOSS OF COOLANT - TWO-INCH (NOMINAL)

PIPE BREAK 

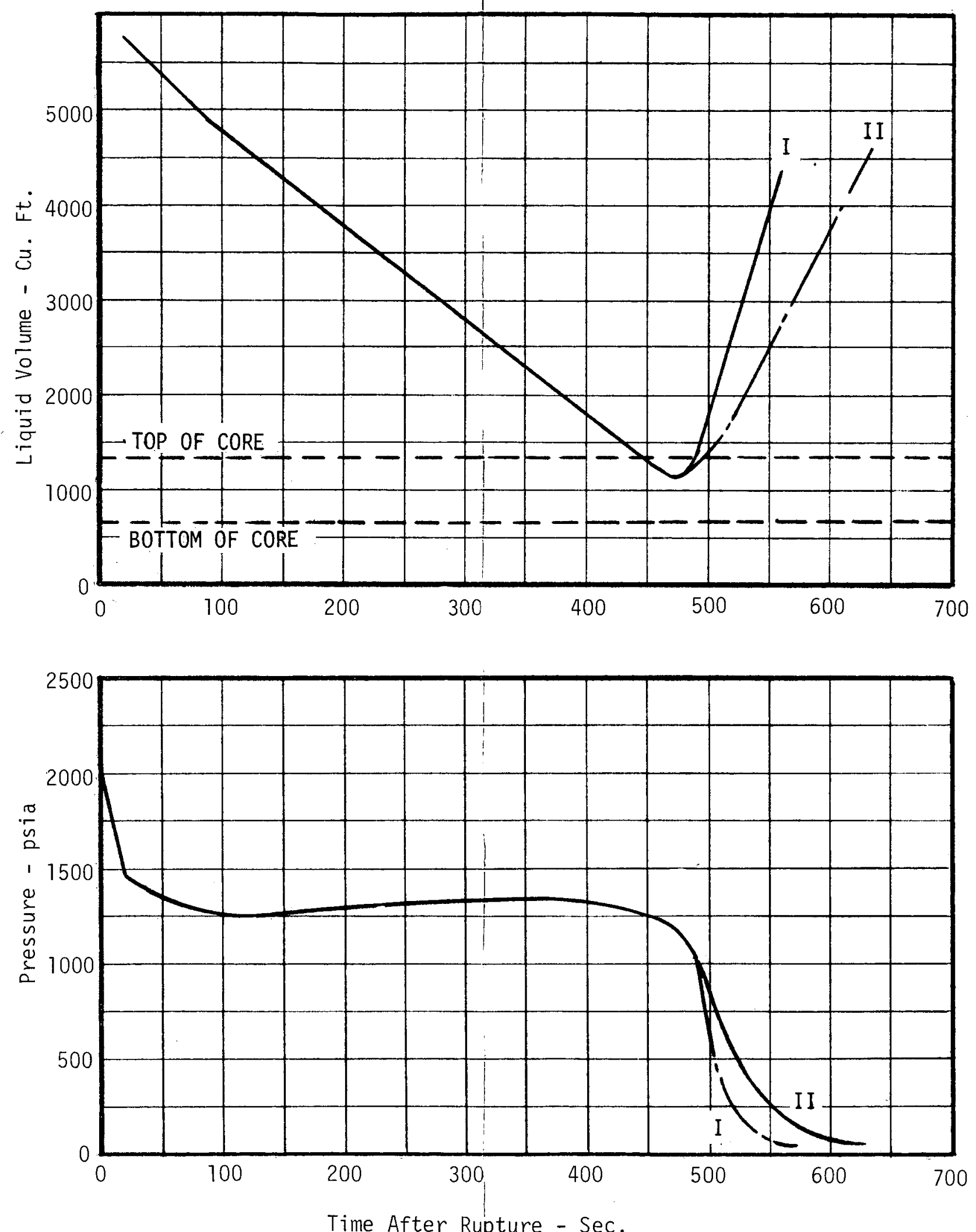

FIGURE 10.4

LOSS OF COOLANT - FOUR-INCH (NOMINAL) PIPE BREAK 

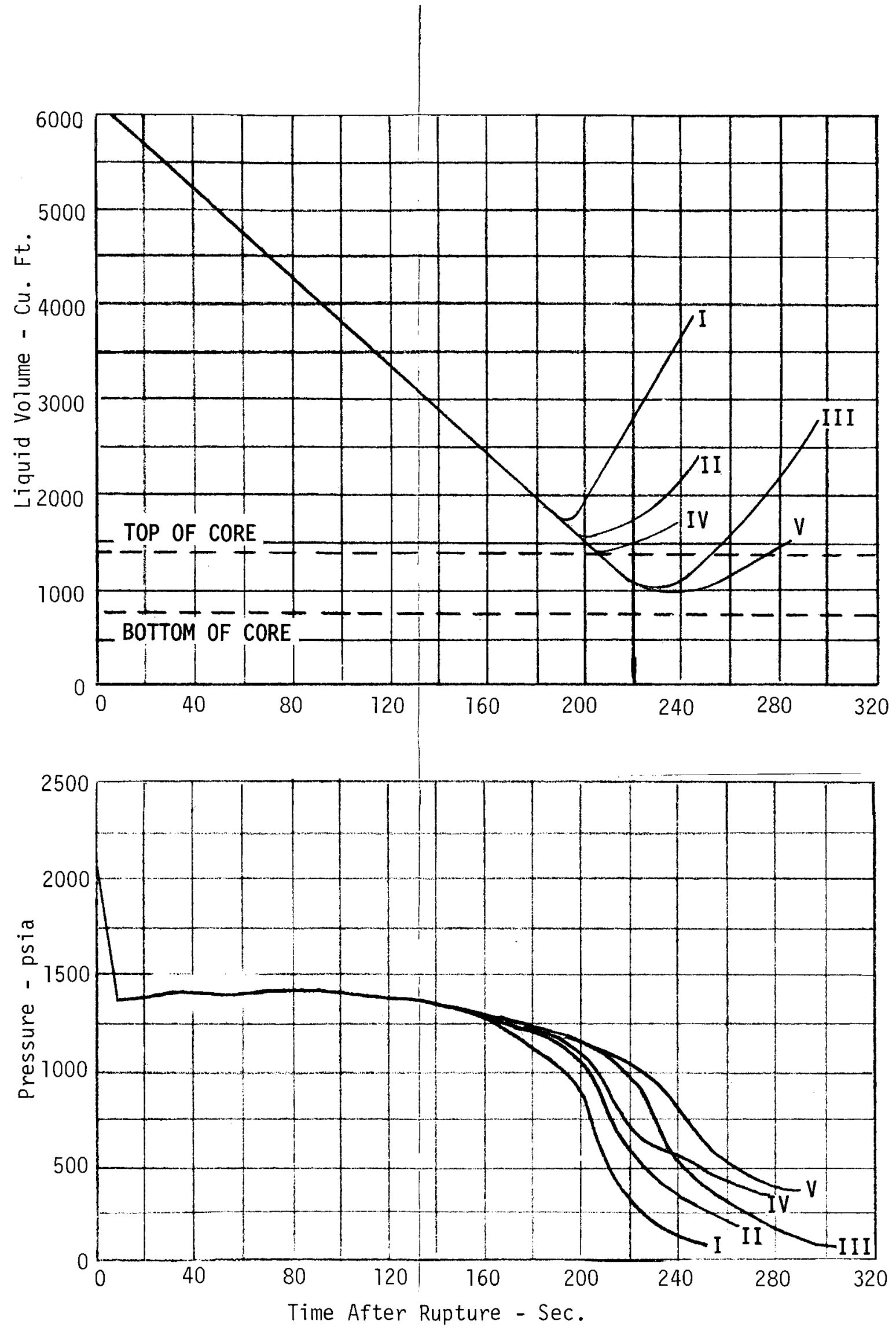

FIGURE 10.5 LOSS OF COOLANT - SIX-INCH (NOMINAL) PIPE BREAK 

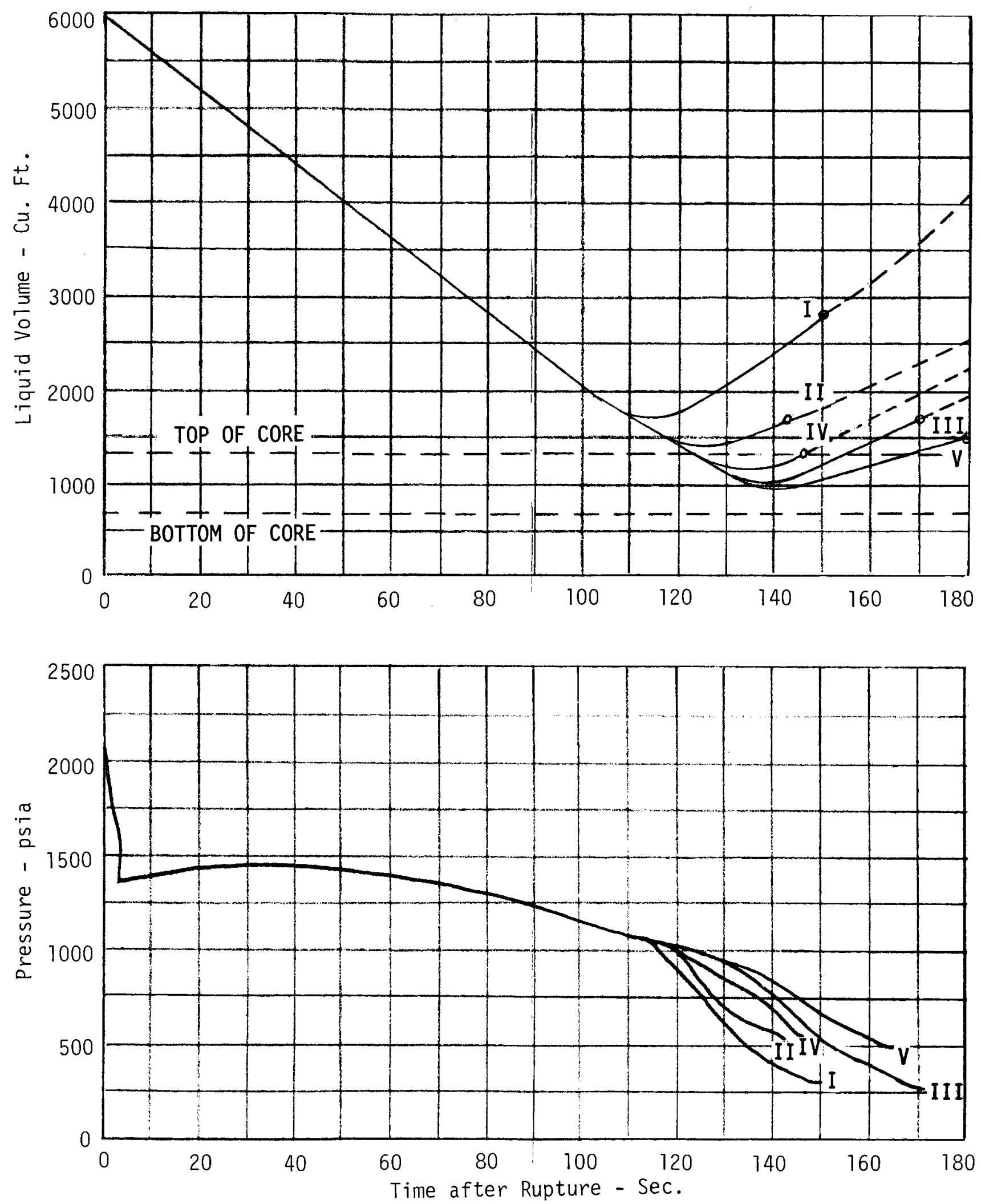

FIGURE 10.6

LOSS OF COOLANT - EIGHT-INCH (NOMINAL) PIPE BREAK 

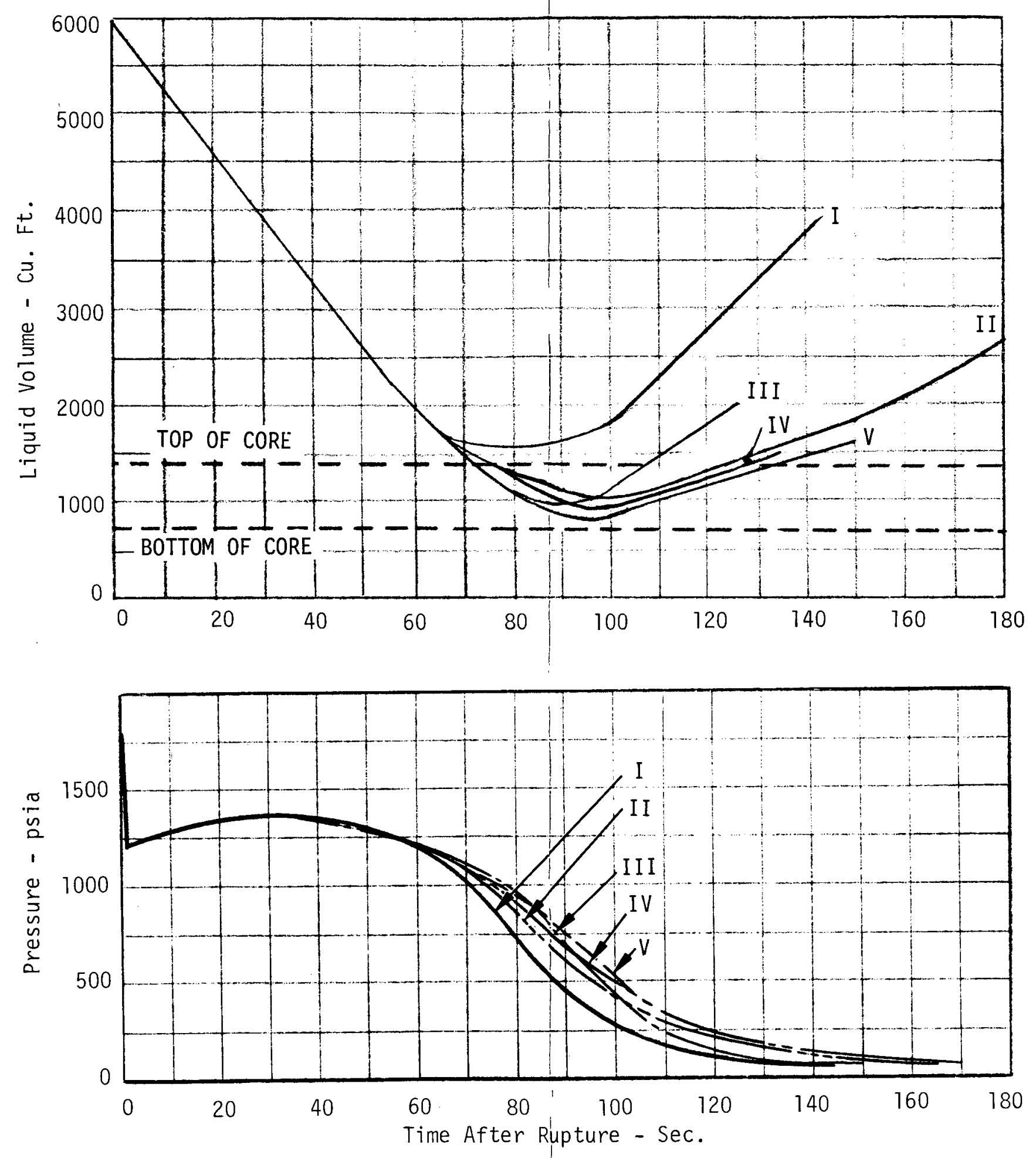

FIGURE 10.7 LOSS OF COOLANT - 10-INCH (NOMINAL) PIPE BREAK 

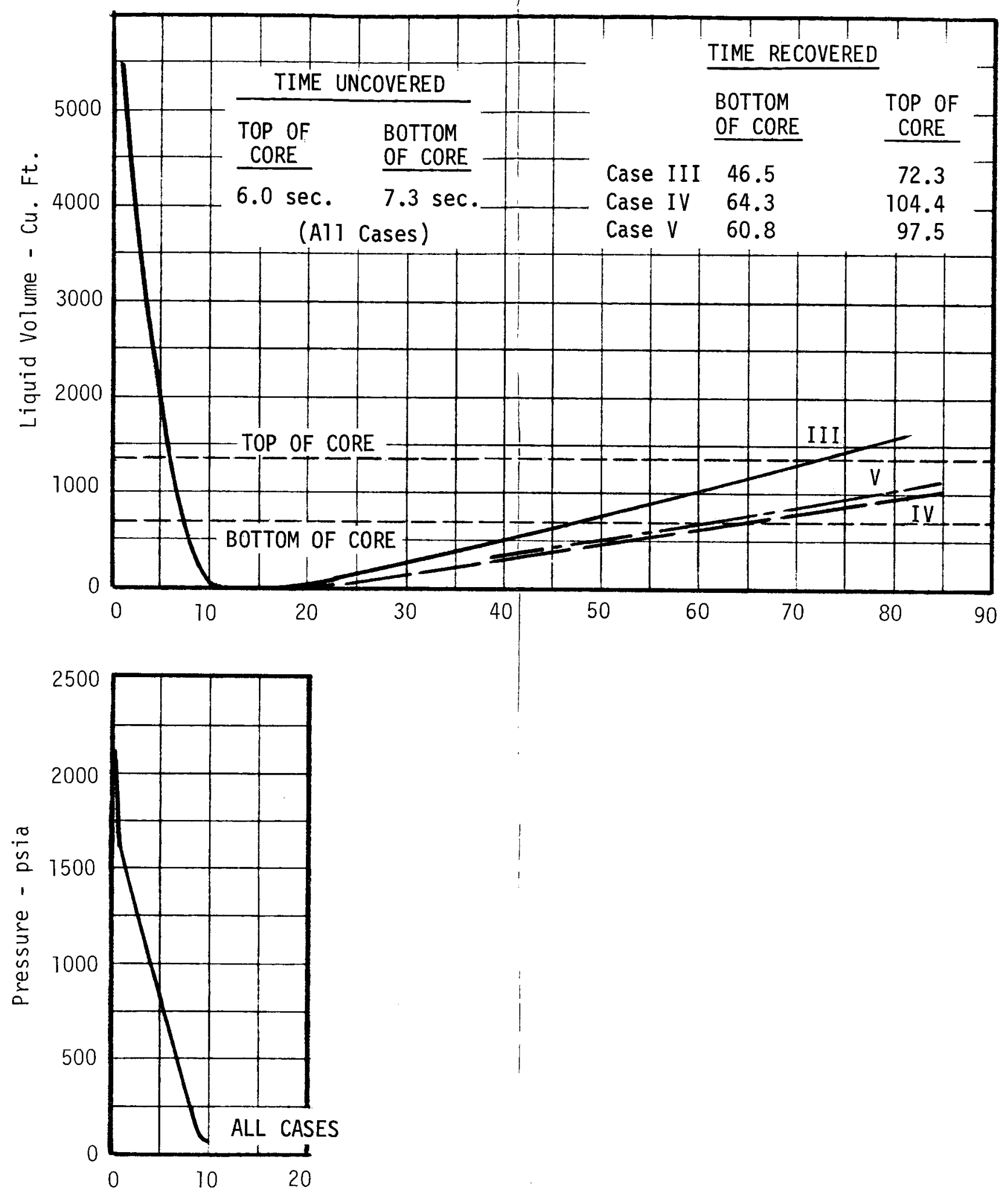

Time After Rupture - Sec.

FIGURE 10.8 LOSS OF COOLANT - 27.5-INCH HYPOTHETICAL MAIN LOOP BREAK 
10.1.4 RESULTS OF BLOWDOWN ANAYLSIS (Continued)

$\begin{array}{ll}\text { Case III, - } & \begin{array}{l}1-, 2-, \\ \text { IV \& and } \\ \text { V }\end{array} \\ & \text { sizes }\end{array}$

Complete loss of the injection flow of one 6-inch injection line cannot be assumed if the break size is less than 6 inches.

It wil1 be noted from the curves that in no case involving a 10 -inch break or smaller does the calculated liquid volume fall below that required to cover the bottom 18 percent of the core. In the worst case $(10$-inch case V), the mid-plane of the core becomes uncovered for 21 seconds, beginning 83 seconds after the break. Assuming the hot-spot power density to exist at the mid-plane, and a surface heat transfer coefficient of $20 \mathrm{Btu} / \mathrm{ft}^{2}-\mathrm{F}-\mathrm{hr}$, the maximum clad temperature reached in this example is calculated to be less than 1,050 F. The calculated short-term bursting temperature of Zircaloy cladding at this condition is greater than $1,100 \mathrm{~F}$, and that of stainless steel cladding is greater than $1,300 \mathrm{~F}$. It is concluded that there would be no clad bursting, hence no fission product release, from either a Zircaloy or a stainless clad core in the accident.

Note that the case which results in maximum core exposure of all of the credible cases, the 10 -inch case $\mathrm{V}$, is extremely improbable. It assumes the occurrence of a 10-inch equivalent break circumscribing a 6 -inch safety injection line juncture and compounds this event with a failure of the flow comparator. The nominal, or "design" case of a 10-inch rupture, which is severance of the 10 -inch pressurizer surge line, is more nearly represented by 10 -inch case IV, in which the core mid-plane is not exposed.

In the hypothetical main loop severance cases, LOCO calculates complete uncovering of the core in 7.3 seconds, and recovery of the bottom, in the worst case (IV), 54 seconds after the break. The mid-plane is recovered 20 seconds later, and the top of the core at 104 seconds after the break. This case represents the minimum degree of core cooling afforded by the safety injection system under the single failure criteria for the hypothetical loss-of-coolant accident. The degree of core damage is assessed in the following paragraph. Note that in the more probable case (III) where both injection trains function and two lines deliver injection flow to the reactor while the third delivers ineffectively to the severed loop, the bottom of the core is recovered in 46 seconds.

\subsubsection{RESULTS OF CORE DAMAGE ASSESSMENT - HYPOTHETICAL LOSS-OF-COOLANT CASE}

In the hypothetical case IV, in which the maximum core exposure occurs, the maximum pellet and cladding temperature were calculated for both stainless steel and Zircaloy clad cores. The fission product release was predicted using the method and data described in Paragraph 13.1.

The fission product activities released to the containment sphere, obtained by the method outlined in Paragraph 13.1 are listed in Table 10.1. The total fission product release for the stainless steel clad core and the Zircaloy clad core corresponds to approximately 6 percent and 25 percent, respectively, of that equivalent to a complete core meltdown. 
Table 10.1 Fission Product Activities Released to Containment Sphere

\begin{tabular}{|c|c|c|}
\hline \multicolumn{3}{|c|}{ DIRECT SOURCES } \\
\hline Energy Group, Mev & \multicolumn{2}{|c|}{ Initial Source Strength, Mev/sec } \\
\hline & $\begin{array}{c}\text { Stainless Stee } 1 \\
\text { Clad Core } \\
\end{array}$ & $\begin{array}{l}\text { Zircaloy } \\
\text { Clad Core }\end{array}$ \\
\hline $\begin{array}{l}0.4 \\
0.8 \\
1.3 \\
1.7 \\
2.5\end{array}$ & $\begin{array}{l}3.2 \times 10^{1.6} \\
2.9 \times 10^{1.7} \\
8.4 \times 10^{16} \\
4.9 \times 10^{1.7} \\
3.3 \times 10^{1.7}\end{array}$ & 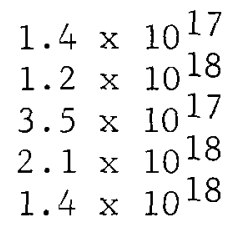 \\
\hline \multicolumn{3}{|c|}{ INHALATION SOURCES } \\
\hline \multirow[t]{2}{*}{ Isotope } & \multicolumn{2}{|c|}{ Initial Activity, Curies } \\
\hline & $\begin{array}{c}\text { Stainless Steel } \\
\text { Clad Core }\end{array}$ & $\begin{array}{l}\text { Zircaloy } \\
\text { Clad Core }\end{array}$ \\
\hline $\begin{array}{l}I-131 \\
I-132 \\
I-133 \\
I-134 \\
I-135\end{array}$ & $\begin{array}{l}1.1 \times 10^{6} \\
1.6 \times 10^{6} \\
2.3 \times 10^{6} \\
2.9 \times 10^{6} \\
2.1 \times 10^{6}\end{array}$ & $\begin{array}{l}4.5 \times 10^{6} \\
6.5 \times 10^{6} \\
9.5 \times 10^{6} \\
1.2 \times 10^{7} \\
8.8 \times 10^{6}\end{array}$ \\
\hline
\end{tabular}

\subsubsection{COOLANT BLOWDOWN CALCULATION}

\section{General Description}

The discharge of reactor coolant from a system rupture is analyzed by means of the digital code LOCO, which solves a series of mass, energy, and volume balance equations for small time intervals defined by small changes in pressure. The results of the computation are expressed as system. pressure and liquid volume remaining as functions of time following the break.

\section{Mathematical Mode 1}

For the purpose of constructing a suitable mathematical model, the blowdown process is separated into four distinct phases:

a) Pressurizer Blowdown - Beginning immediately after the occurrence of the rupture and continuing until the pressurizer is emptied of liquid. 


\subsubsection{COOLANT BLOWDOWN CALCULATION (Continued)}

b) Subcooled Blowdown - Consisting of the further expansion of the reactor coolant to saturation pressure conclitions.

c) Two-phase Blowdown - Starting when the reactor coolant reaches saturation condition and two-phase flashing flow begins. This phase continues at least until the steam-water interface falls below the level of the break.

d) Steam Blowdown - Beginning when the interface falls below the break, if water entrainment is low. Steam flow continues until the pressure in the Reactor Coolant System reaches equilibrium with the pressure in the vapor container.

A more detailed description of each phase, and the assumptions and methods used in the calculations are presented below.

Phase 1 - Pressurizer Blowdown

The first phase begins when a rupture occurs, exposing the interior of the reactor coolant system to the low-pressure environment of the containment sphere. At this instant the water in the system is in a sub-cooled state. The first discharge through the break will thus consist of compressed water, impelled by the expansion of the steam volume in the pressurizer. This phase continues until the pressurizer is empty of liquid. It is assumed that the coolant comes to its weighted average temperature and thus, the only significant pressure gradient in the system is at the break. Other friction effects, including the losses in the pressurizer surge line, are neglected.

Phase 2 - Sub-Cooled Blowdown

At the end of the first phase the pressure in the entire system is higher than the saturation pressure of the remaining reactor coolant. The second phase consists of the expansion of the coolant to saturation pressure. The only difference between this phase and the previous one is a shift in emphasis from the pressurizer to the reactor coolant system proper. Actually, the two phases may occur with some overlapping, but in order to achieve more flexibility in the calculation, they are separated. The division makes it possible to instruct the code to ignore the pressurizer blowdown phase for the calculation of conditions following a break of the pressurizer surge line.

At some time during the first or second phases a reactor trip is initiated by a low-pressure signal. The trip results in insertion of control rods. Until the trip is completed, heat addition to the system continues at 100 percent of rated core power, and is assumed to be removed at the same rate by the steam generators. After the trip, the nuclear heat input for each interval of the calculation is computed by a special subroutine using the residual heat relations. 


\subsubsection{COOLANT BLOWDOWN CALCULATION (Continued)}

Where

$$
\begin{aligned}
& r(t)=0.051 e^{-0.004 t}+0.177 e^{-0.1 t} \text { for } t<60 \mathrm{sec} \\
& r(t)=0.28+0.023 \mathrm{e}^{-0.009 t} \text { for } t>60 \mathrm{sec}
\end{aligned}
$$

A11 of the sensible heat in the core and one-third of the vessel and thermal shield is assumed to be transferred to the coolant with a constant temperature difference between the core and coolant.

The rate of discharge from the system during phases 1 and 2 is calculated for each interval by the use of the following equation based on the investigation of Burnell and others: (J. G. Burnell - "Flow of Boiling Water Through Nozzles, Orifices and Pipes," Journal, The Institution of Engineers, Australia, March 1946.)

$$
G=\sqrt{2 g \rho\left[P-(1-C) P_{\text {sat }}\right]}
$$

The surface tension factor $\mathrm{C}$ is determined from an empirical expansion given by Burne11. Fluid properties are obtained from standard steam table subroutines included in the code.

Phase 3 - Two Phase Blowdown

In the third phase the nature of the discharge changes radically, as flashing flow occurs at the break and a two-phase mixture flows through the hole. For most of this phase the pressure difference across the break will exceed the critical pressure ratio and choking flow will exist. It is interesting to note at the beginning of this phase, if the discharge through the break is smal1, the pressure in the system may actually rise because the volume of steam generated by the residual heat production is greater than the volume leaving the system through the break.

The discharge rates for two-phase flow are calculated using an equation derived from the general energy equation by Benjamin and Miller.

(M. W. Benjamin and J. G. Miller - "The Flow of a Flashing Mixture of Water and Steam through Pipes," Transactions ASME, October 1942.)

$$
G^{2}=\frac{-\int_{P_{1}}^{P_{2}} \frac{d P}{v}}{\ln \left(\frac{v_{2}}{v_{1}}\right)+\frac{f L}{2 D}}
$$

where the subscripts 1 and 2 refer to the beginning and end at the flow path, respectively. 


\subsubsection{COOLANT BLOWDOWN CALCULATION (Continued)}

This equation is based on the assumption of a homogeneous flow of the two phases (no slip). At low pressures, where slip does occur, the assumption is of questionable validity. However, the results are correspondingly conservative and represent an upper limit for flashing flow. Note that this equation includes a term $\frac{f 1}{2 D}$ for pressure losses in the piping and components. This information is supplied as part of the input data. To be certain that the blowdown calculation is conservative, the total resistance used consists entirely of the discharge coefficient at the break.

Generally speaking, the pressure at the end of the path, $\mathrm{P}_{2}$, is the critical pressure and is unknown. An iterative subroutine is therefore used in the code to determine the maximum (critical) flow rate for each interval for which the calculation is performed.

Throughout the first three phases, credit is taken for heat removal by the steam generators. The reactor decay heat in the energy balance equations is replaced by the difference between the reactor decay heat and steam generator heat removal rate. Steam generator heat removal continues until either the reactor or turbine cycle side of the steam generator is emptied of water.

\section{Phase 4 - Steam Blowdown}

For small breaks the mechanism of two-phase flow will cease when the steamwater interface falls below the break. After this time the flow consists of saturated steam carrying some amount of entrained water.

The LOCO code calculates the liquid volume point at which the steam blowdown begins. The criterion for steam discharge is that no more than one percent liquid be entrained in the steam flow from the liquid space in the reactor vesse1.

For pure steam flow the following equation is developed for an adiabatic expansion from the boiling interface to the break:

$$
G=.94 \sqrt{2 g \rho^{2} J\left(h_{1}-h_{2}\right)}
$$

Since the flow passes through what may be considered an orifice, a flow coefficient of 0.94 has been included in the theoretical flow equation. This is a conservative value and will yield higher flow rates than would actually be expected. 
10.1.6 COOLANT BLOWDOWN CALCULATION (Continued)

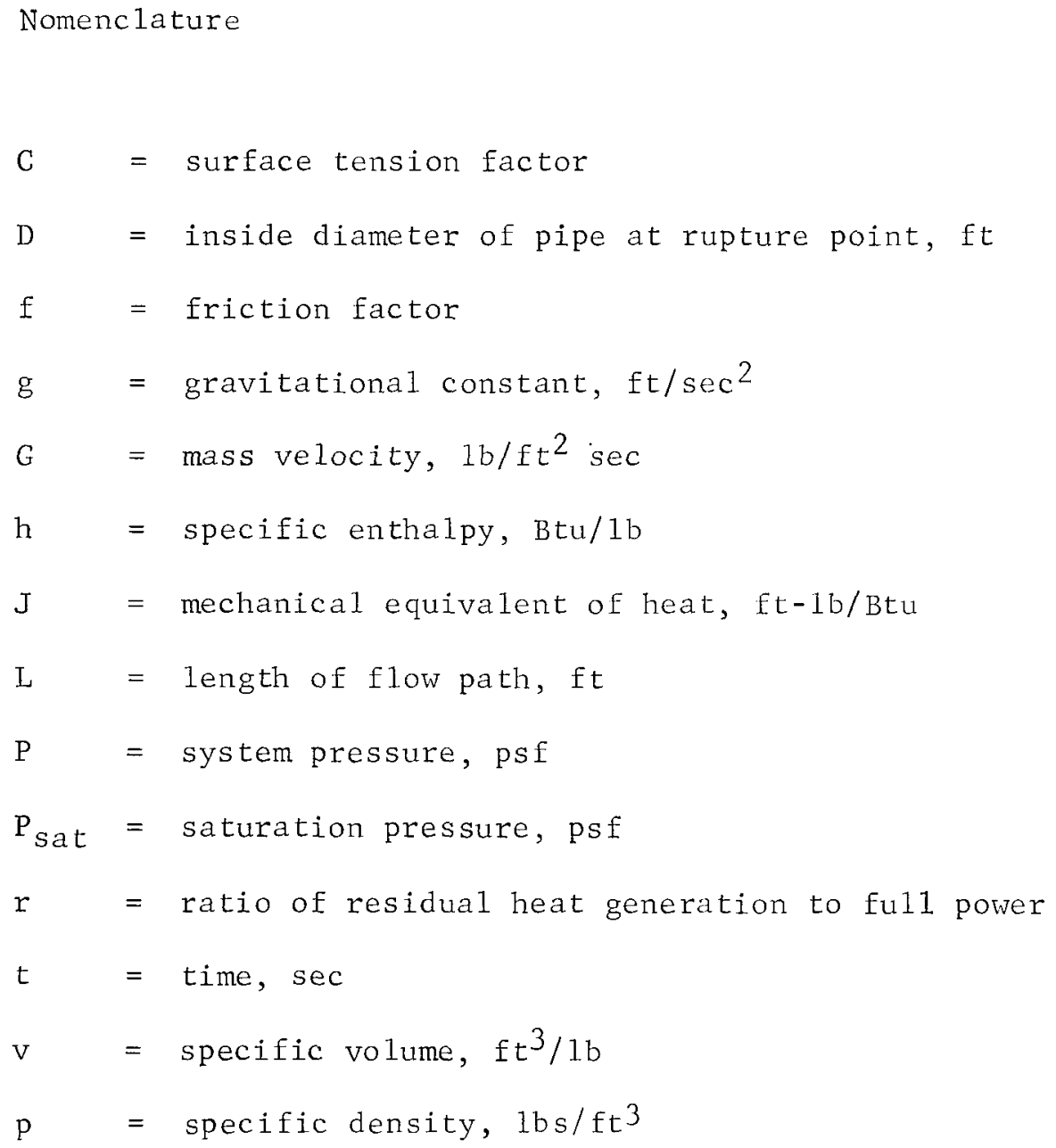




\subsection{LOSS OF COOLANT FLOW}

\subsubsection{GENERAL}

The response to a reduction in coolant flow while the reactor is at substantial power is a corresponding increase in the reactor coolant temperature. If the increase in coolant temperature is large enough, DNB will occur. Depending on the heat $f 1$ ux and flow conditions at the point of DNB, cladding temperature rise may result in plastic deformation or rupture unless corrective action is taken. To avoid such damage, a reactor trip is actuated under those conditions of reduced flow and initial heat flux which could result in excessive cladding temperature. In this context, excessive temperature is that which might result in plastic deformation of the cladding.

Public safety is not affected by a loss of flow, even if clad damage should occur, since any activity released from the fuel is retained in the Reactor Coolant system. Independent reactor trips are provided that would terminate the coolant temperature rise without exceeding system design pressure. Instrumentation provided to detect the loss of flow condition and initiate reactor trip is discussed in Paragraph 3.3.

A loss of coolant flow would be initiated by loss of voltage on the pump supply buses, a circuit breaker trip, an electrical or mechanical failure in one of the pumps or pump motors, or by an operator manually tripping a pump when reactor power is above the prescribed limit for such action.

\subsubsection{FACILITY DESIGN ASPECTS}

In designing the reactor coolant pumps, the flywheel inertia was selected on the basis of providing sufficient coastdown flow to protect the fuel cladding in the case of coincident loss of power to all three pumps with immediate reactor trip from full load. After pump coastdown is completed, natural circulation of coolant through the core and reactor coolant loops builds up due to the thermal syphoning effect of the reactor and steam generator. The resulting flow rate is sufficient to remove the residual heat of the core without damage to the fuel or cladding.

If the loss of flow is caused by a loss of bus voltage, the reactor is tripped by the low bus voltage pump circuit breaker trip or the low flow trip circuits. If the loss of flow results from a pump breaker being opened by the operator, the breaker trip or the low flow trip circuits would again trip the reactor. A pump failure without a breaker trip would actuate the low flow trip circuit in the affected loop.

The low bus voltage trip circuit consists of two detection channels, one on each reactor coolant pump bus, which opens a pump breaker on detection of loss of bus voltage. The pump circuit breaker trip circuit and low flow trip circuit each consist of one detection channel per reactor coolant loop. Above the loss of flow permissive power level, a signal from either of these circuits indicating a loss of flow in any one loop will actuate a reactor 


\subsubsection{FACILITY DESIGN ASPECTS (Continued)}

trip; below this power level, coincident signals from either circuit in any two loops are required to actuate a trip.

Either the circuit breaker trip or the low flow trip will protect against core damage in any loss-of-flow transient. If none of these circuits were assumed to function, coolant temperature would increase until the variable low pressure trip, the high pressure trip, or high pressurizer level trip points were reached. In such case, the Reactor Coolant System would not be overpressured. Each of these circuits consists of three channels; signals from any two out of three in coincidence will actuate a reactor trip.

Finally, manual reactor trip by the operator is an additional means of limiting the consequences of loss of flow, independent of the automatic trip schemes.

\subsubsection{METHOD OF ANALYSIS AND SELECTION OF PARAMETERS}

The loss of coolant flow accident is analyzed by a combination of computational techniques. A full plant simulation is used to compute the core average and hot spot heat flux transient responses including flow coastdown, temperature, reactivity and control rod reactivity insertion effects. These flux responses are used as the input to a digital computer code CAT II. This code is described by R.0. A Sandberg in "CAT II - An IBM 7090 Code for Predicting Thermal and Hydraulic Transients in an Open Lattice Core", WCAP-2059. The code performs a detailed thermal-hydraulics computation to compute the margin to DNB using the continuity, momentum and energy equations of fluid flow together with the W-3 DNB correlation described in Paragraph 3.3 .

For a trip initiated from a turbine protective device or a reactor protective device, the generator is maintained connected to the electrical system for one minute. The reactor coolant pumps remain connected to the generator after the one minute delay. Continued forced circulation is maintained on turbine-generator inertia for three to four minutes.

In order to assure the conservatism of the results, the following assumptions are made concerning the reactor conditions at the start of the transient:

a) Operating point - The operating point is assumed to be most adverse with respect to the margin-to-DNB. The conditions are the maximum steady state power level, minimum steady state prinary pressure, and maximum steady state core water inlet temperature are as follows:

$$
\begin{aligned}
& \text { Power }=(1.03)(1,347 \mathrm{Mwt}) \\
& \text { Pressure }=2,100-30=2,070 \text { psia } \\
& \text { Inlet }=553+4=557 \mathrm{~F}
\end{aligned}
$$




\subsubsection{METHOD OF ANALYSIS AND SELECTION OF PARAMETERS (Continued)}

b) Moderator and Doppler Coefficient - The smallest negative expected value of Doppler reactivity coefficient and the most positive expected value of moderator reactivity coefficient are assumed since these result in the minimum hot spot heat flux decrease during the initial part of the transient, the time when the mininum DNB ratio is reached. The low flow trip is assumed to be initiated at 90 percent of full flow. The reactor trip is completed in less than three seconds, however, and these temperature feedback effects are not very significant.

$$
\begin{aligned}
& \alpha_{\mathrm{f}}=-1 \times 10^{-5} \delta \mathrm{kF} \\
& \alpha_{\mathrm{w}}=+0.5 \times 10^{-4} \delta \mathrm{kF}
\end{aligned}
$$

c) Reactor Trip - Since the low flow trip results in a longer delay than the bus undervoltage or breaker trips, it is the low flow trip which is assumed to be actuated. After the trip signal is given, a conservative instrumentation delay is assumed before the rods are inserted. The assumptions for reactor trip are summarized in Appendix A. Credit is taken for the reactivity added during the rod run-in time. Minimum available reactivity worth in withdrawn rods is assumed, and the most reactive rod is assumed to be stuck in its fully withdrawn position. The trip worth is four percent $\delta k$ for this case.

d) A conservative residual heat rate equal to eight percent of full power is assumed. The reactor inlet temperature is assumed constant. This is a reasonable assumption, since the minimum DNB ratio in the core is reached in about three seconds, which is shorter than the transport time between the steam generator outlet and the core inlet.

\section{2 .4 RESULTS}

The reactor coolant flow coastdown curve, shown in Figure 10.9, was determined using a conservative high estimate of loop pressure losses. The extended coastdown time illustrates the effect of flywheel inertia added to the reactor coolant pump. The reactor trip is completed in about three seconds where there is still between 70 and 80 percent of full flow. There is no approach to DNB, even for the complete and simultaneous loss of power to a11 the reactor coolant pumps.

Figure 10.10 shows the neutron power response, while Figure 10.11 shows the average channel and hot channel heat flux response. The effect of the time delay associated with the fuel to water heat transfer is evident when compared to the neutron power response.

Figure 10.12 shows the DNB ratio as a function of time at the point of minimum DNBR, 65 percent up the channel from the core inlet. It is seen that the minimum DNB ratio is 1.62 , which is well above the 1.30 limit.

The loss of flow in one or two loops from full power operation is less severe because there is more total core flow during the same core heat flux conditions following reactor trip. 


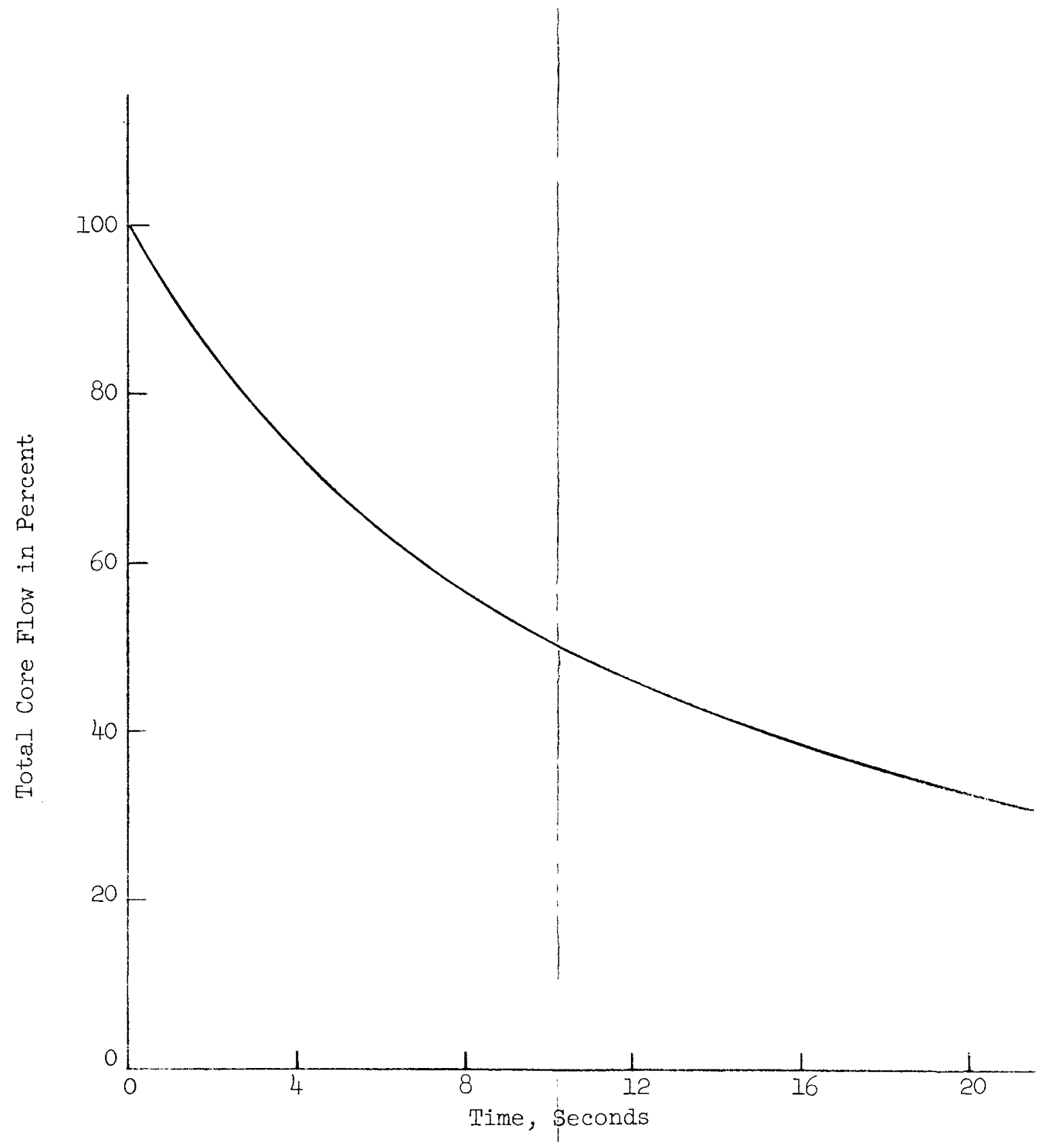

FIGURE 10.9 REACTOR COOLANT FLOW FOLLOWING COMPLETE LOSS OF PUMP POWER 


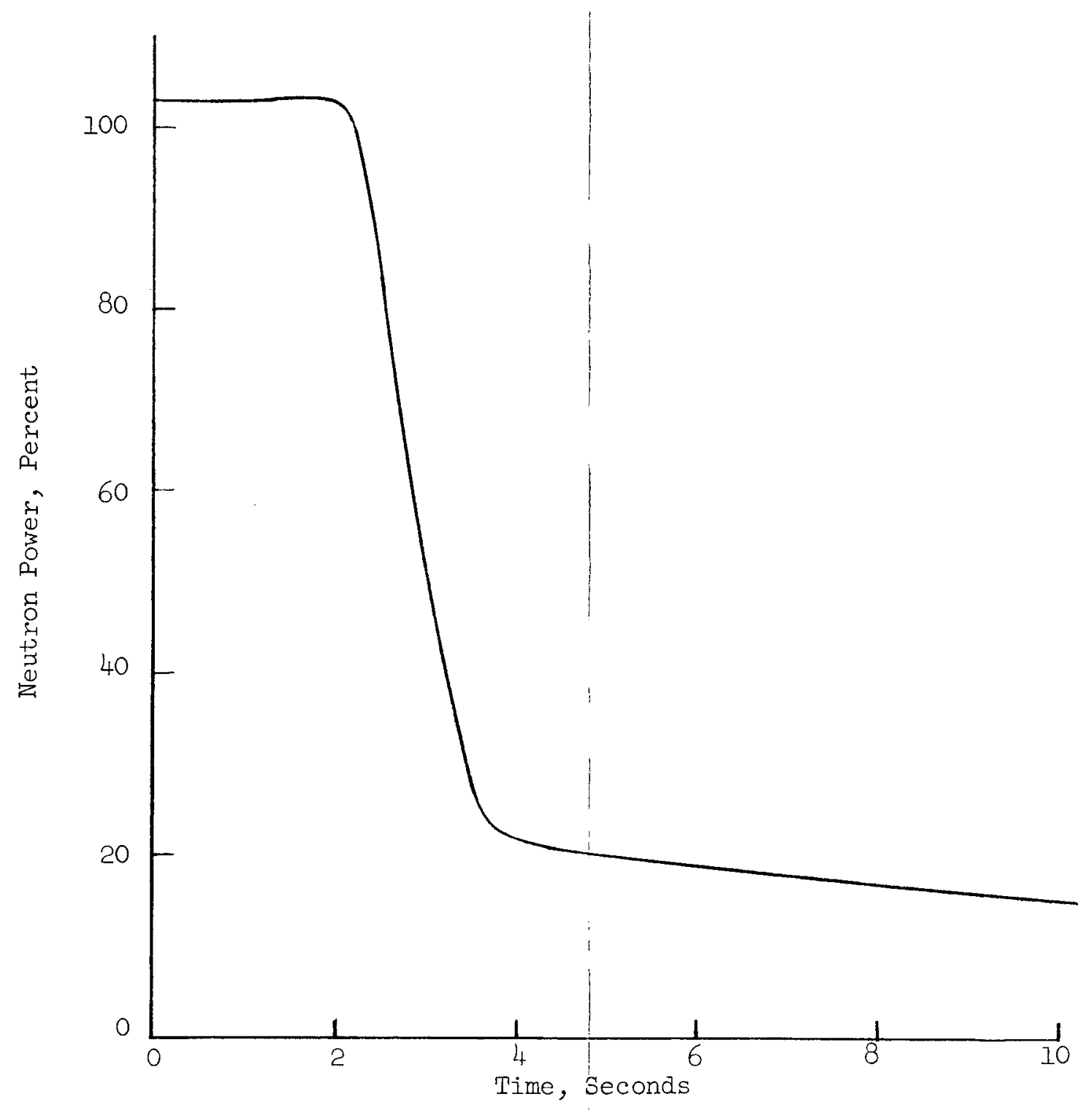

FIGURE 10.10

NEUTRON POWER FOLLOWING COMPLETE LOSS OF PUMP POWER WITH REACTOR

TRIP 


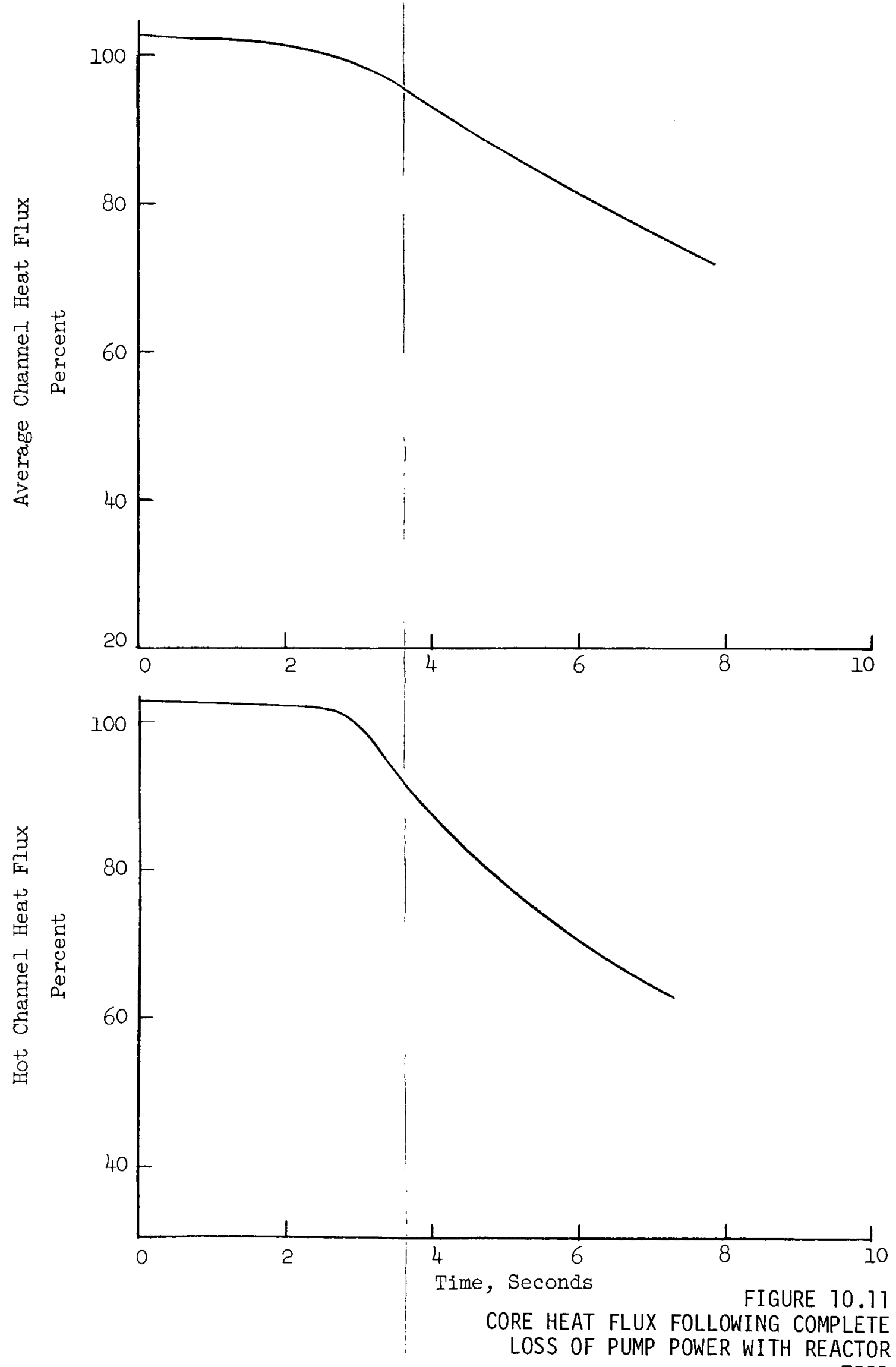

TRIP 


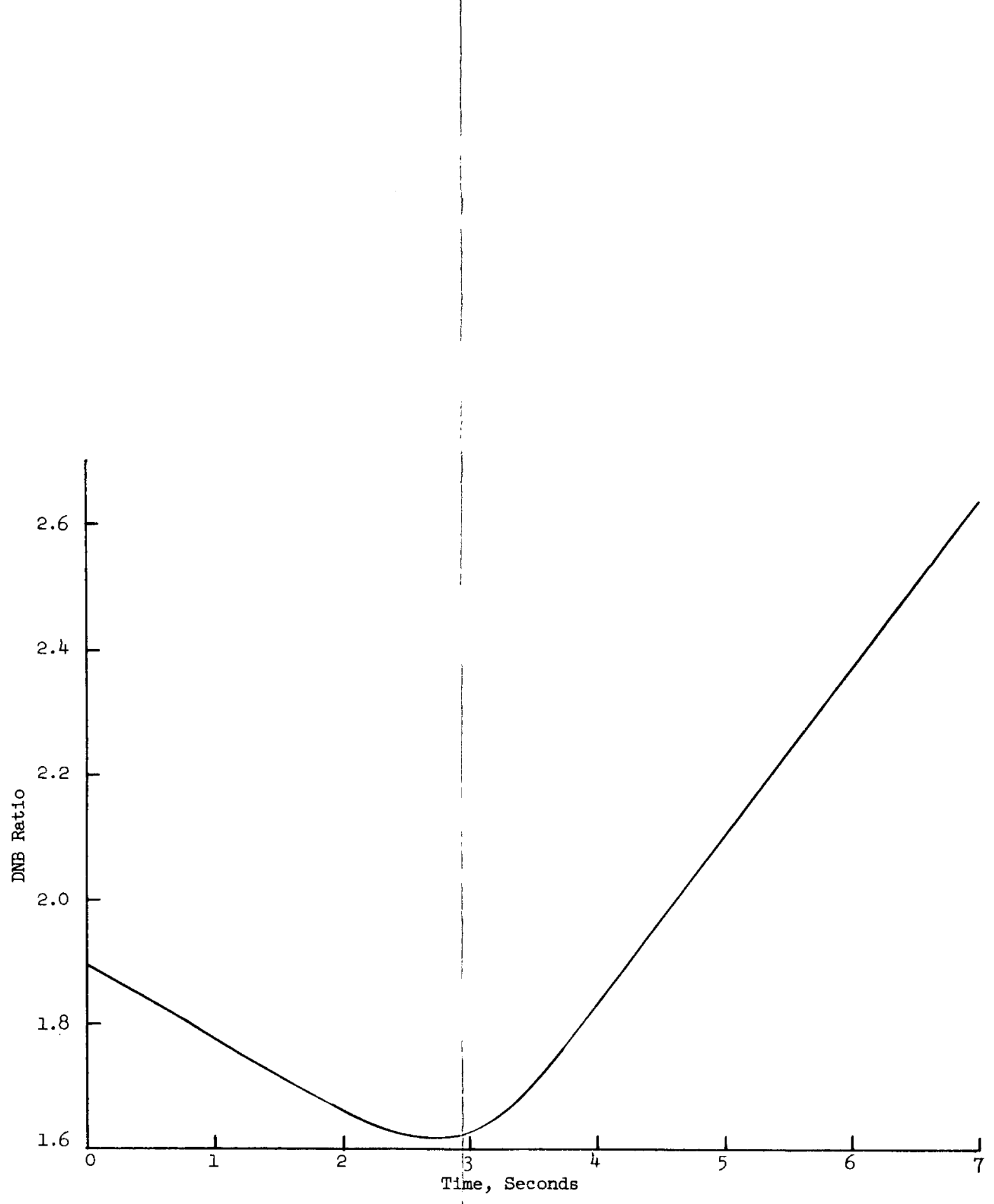

FIGURE 10.12 MINIMUM DNB RATIO VERSUS TIME FOLLOWING COMPLETE LOSS OF PUMP POWER WITH REACTOR TRIP 


\section{2 .5 CONCLUSIONS}

The core is protected from DNB for the transient resulting from a complete loss of power to all three reactor coolant pumps while operating at the maximum initial steady state operating conditions. This protection is provided by a direct reactor trip on loss of flow, combined with the extended flow coastdown provided by high inertia reactor coolant pumps. 


\subsection{FUEL HANDLING}

\subsubsection{GENERAL}

In the design of reactor components, fuel handling facilities, and fuel handling methods, numerous precautions have been taken to preclude accidental nuclear excursions, release of fission products, and overexposure of personnel during refueling. Safeguards have been adopted with particular attention to the following potential sources of hazard:

\footnotetext{
- Improper withdrawal of rod cluster control assemblies

- Failure to maintain subcritical geometry

- Failure to maintain adequate shielding for personnel

- Failure to maintain adequate cooling of fuel assemblies

- Mechanical damage to cladding
}

Al1 refueling operations will be conducted in accordance with prescribed procedures under direct surveillance of a technically-trained supervisor.

Before any refueling operations begin, shutdown procedures for the Safety Injection system will be completed. The procedures are discussed in Paragraph 5.1 .

Monitors equipped with audible alarms, will be maintained in continuous operation in the immediate work vicinity to advise personnel of any increased activity.

\subsubsection{FUEL HANDLING PRECAUTIONS}

Fuel handing precautions presented in this paragraph are in addition to those contained in Paragraph 3.9, Fuel Handling and Transfer System, and Paragraph 3.2, in Reactor Mechanical Design.

\section{Control Rod Cluster Withdrawa1}

The following safeguards will be employed to avoid accidents due to inadvertent rod withdrawal:

a. Prior to disconnecting drive mechanism power supplies, the drive mechanism will be disengaged from the drive shaft.

b. Prior to removal of the reactor vessel head, boron concentration of water in the reactor and its coolant loops will be increased to refueling concentration and verified by analysis. Refueling concentration is sufficient to maintain the fully-loaded, cold, clean core subcritical by 10 per cent $\Delta \mathrm{k} / \mathrm{k}$ with all rod cluster control assemblies inserted. This concentration is sufficient to maintain the core subcritical even if all rods are removed. 


\subsubsection{FUEL HANDLING PRECAUTIONS (Continued)}

c. During removal of the reactor vessel head, a continuous audible signal from source range nuclear instrumentation will be monitored to verify that the core is not approaching a critical multiplication factor. As the vessel head is raised, a visual check is made to verify that the drive shafts are free in the mechanism housings.

d. After the vessel head is removed, the control cluster drive shaft assemblies are removed from their respective control clusters using the manipulator crane and special drive shaft unlatching tool. A spring scale will be used to determine that the drive shaft is free of the control cluster as the lifting force is applied.

e. During reactor refueling operations, a continuous audio count rate signal from the source range nuclear instrumentation will be provided in the control room and within the sphere in the refueling cavity area.

Transfer and Storage of Spent Fuel Assemblies

Storage racks in the spent fuel pit are arranged so that spent fuel assemblies cannot attain a critical geometry even with unborated water in the spent fuel pit. This is applicable in storage, during transfer, or in the process of loading the fuel shipping cask. The racks are spaced in a square pitched pattern, 20 inches on centers with 12 inches between assemblies. Water in the spent fuel pit is borated to $2,900 \mathrm{ppm}$ because there can be an interchange of water with the refueling cavity.

\section{Water Shielding}

Fuel handling manipulators and hoists are designed to reach their physical limit on lift before raising a fuel assembly or control cluster above a height consistent with adequate water shielding. This safeguard applies to handing facilities in both the Containment Sphere and the spent fuel pit area. Radiation levels at the refueling water surface in the spent fuel pit and in the refueling canal are monitored continuously to warn personnel of any increased activity.

\section{Heat Removal}

Adequate cooling of fuel during underwater handing is accomplished by convective heat transfer to the surrounding water, where the assembly is immersed continuously while in the refueling cavity or spent fuel pit. Analysis has shown that forced circulation through the transfer tube is not required. In the event the carrier, containing a spent fuel assembly, became stuck in the transfer tube, adequate cooling will be maintained by natural convection. If some boiling did occur, there would be no danger of cladding failure at the existing conditions.

Protection Against Mechanical Damage

Gripping devices and handing fixtures used to lift fuel assemblies and 


\subsubsection{FUEL HANDLING PRECAUTIONS (Continued)}

other core internals are designed to avert accidental breach of cladding during fuel handling. Equipment is designed to withstand seismic stresses of $0.5 \mathrm{~g}$ in the horizontal and vertical directions resulting from ground accelerations. The new fuel and spent fuel assembly handling tools are designed so that engagement of the fuel assembly is accomplished with positive, cam-actuated latching fingers. A manually-operated, spring-loaded safety catch must be actuated to ensure positive engagement with the assembly before it can be lifted. The manipulator crane gripper is monitored by an electric load cell indicator which will stop the winch drive from moving in the up direction if the load exceeds a preset value. The gripper is also interlocked through the weight sensing device and a mechanical spring lock to prevent opening when supporting a fuel assembly.

Further protection against an accidental breach of cladding is provided by making it impossible to pass the fuel shipping cask above the spent fuel storage rack locations.

\subsubsection{ASSUMPTIONS AND METHOD OF ANALYSIS}

Due to fuel handling precautions, actual operating experience, and the inherent strength of fuel assemblies and individual fuel rods, it is highly improbable that a fuel handling accident involving a breach of clad integrity will occur. Even if clad damage was assumed to occur, the potential hazard would be small because of the low temperature of $\mathrm{UO}_{2}$ (enhancing its retentivity for volatile fission products) and the tendency of the refue1ing water to absorb any non-gaseous fission products which might escape as the gas bubbles through it.

For purposes of analysis, it has been assumed that an entire row of fuel rods (14) has been damaged and the cladding breached. It takes approximately 90 hours after a reactor shutdown before a fuel assembly is accessible for removal from the core. The resulting fuel rod gap noble gas activity release is based on data presented in Paragraph 13.1, Fission Product from Fue1.

\section{3 .4 RESULTS}

The environmental consequences of the noble gas activity noted below are presented in Paragraph 15.2, Other Malfunctions. 
10.3.4 RESULTS (Continued)

Results of noble gas tests conducted are as follows:

I. $\quad E \leq 0.4 \mathrm{Mev}$

$4.26 \times 10^{13}$

II. $0.4 \mathrm{Mev}<\mathrm{E} \leq 0.8 \mathrm{Mev}$

$8.0 \times 10^{7}$

III. $0.8 \mathrm{Mev}<E \leq 1.7 \mathrm{Mev}$

Negligible

IV. $E>1.7 \mathrm{MeV}$

Negligible 


\subsubsection{GENERAL}

The integrity of the turbine cycle steam systern affects the safety of the plant in two principal ways:

a) A rupture in this system increases the rate of heat extraction by the steam generators and causes cooldown of the reactor coolant. If the nuclear power required to meet this apparent load increase is excessive, or if the cooldown and depressurization result in inadequate subcooling, the reactor is tripped to ensure the minimum desired margin to DNB in this phase of the transient.

b) Cooldown after trip, due to continued discharge of steam, reduces the shutdown reactivity margin. In the more severe cases, injection of borated water is required to terminate this reactivity transient within safe 1 imits.

In both respects, analysis of the conditions ensuing from a steam break shows that the safety of the general public is assured by the continued integrity of the Reactor Coolant System barrier, and that the possibility of core damage exists only in the most extreme and highly improbable cases. The analysis covers the full range of possible break sizes, up to and in including severance of main steam piping at the point which yields the highest postrupture rate of steam flow. All cases of uncontrolled steam discharge by means other than pipe rupture, for example improper opening of the steam dump or bypass valves, are well within this range.

\subsubsection{FACILITY DESIGN ASPECTS}

\section{Effect of Steam Break}

A steam break is reflected in the Reactor Coolant System as a sudden increase in turbine cycle load causing a reduction in Reactor Coolant System temperature, pressure and pressurizer leve1. During the first phase of the transient, the reactor control system responds to increase reactor power in attempting to maintain constant average temperature. Normal overpower trip protection will terminate this power increase without core damage. If the break is so large that the reactor control system cannot compensate for the decrease in temperature and pressure, a low pressure reactor trip may occur before the overpower trip.

During the second phase of the transient, which follows reactor trip, continued steam flow through the break further reduces the Reactor Coolant System temperature, pressure, and pressurizer level. With a negative moderator coefficient, an increase in reactivity accompanies this cooldown, thereby reducing the shutdown margin. Transfer of the reactor coolant makeup control to the borate mode could be made in order to help compensate for this reactivity gain. 


\subsubsection{FACILITY DESIGN ASPECTS (Continued)}

If the cooldown rate is sufficiently rapid, coolant contraction will cause the coincident low pressurizer pressure and low pressurizer level condition which automatically initiate borated water addition by the Safety Injection system. Injection continues until the pressurizer is refilled with water or until the reactor coolant pressure rises to the shutoff pressure of the Safety Injection System. Regardless of break size, the amount of boron injected into the system in this manner is sufficient to maintain the core in a subcritical condition.

\section{Effect of Break Inside Containment Sphere}

If the break is inside the containment sphere, the Reactor Coolant System cooldown is terminated when the steam pressure in the steam generator approaches the equilibrium containment sphere pressure. If the break is outside the sphere, cooldown is terminated when the water in the feedwater side of all steam generators is completely boiled away.

For steam breaks ranging from small size up to that equivalent to the inadvertent opening of all steam dump valves, the protection described above prevents core damage in all cases, including those which combine the following most adverse conditions:

1. Highest worth control rod stuck in the withdrawn position

2. Most negative moderator coefficient

3. Minimum shutdown margin available with the remaining control rods

To minimize the probability of this transient accurring due to inadvertent opening of steam dump valves, the air operators of these valves are designed so that loss of supply air pressure would cause these valves to remain closed. An interlock is provided in the steam dump controller to reduce the probability of a spurious steam dump. This interlock requires a large turbine-generator load decrease in coincidence with high reactor coolant average temperature to actuate steam dump.

Under nominal conditions, even the highly improbable case of a main steam pipe severance would not result in core damage. Should a very large area break occur in coincidence with the adverse conditions itemized above, however, a momentary return to criticality could occur. If the flux distribution resulting from the single stuck rod is sufficiently distorted, DNB may occur, causing clad deformation and possible bursting in a limited core region. Public and plant personnel safety is assured by the fact that the transient is self-limiting (rising temperatures, coolant voids, and continued boron addition act to terminate the transient), and by the fact that any fission products released are confined to the Reactor Coolant System.

\subsubsection{ASSUMPTIONS AND METHOD OF ANALYSIS}

Steam break analysis is performed by utilizing a detailed analog simulation of the plant. 


\subsubsection{ASSUMPTIONS AND METHOD OF ANALYSIS (Continued)}

The steam flow through the break is determined as a function of steam generator pressure, with allowance for pressure losses up to the location of the break. Critical flow occurs at the break during most of the transient. The flow is proportional to steam generator pressure. The station operates with an increasing steam pressure during decreasing load. Thus the maximum initial steam flow through the break occurs with the plant at low power.

The transient temperature, power and pressure behavior of both the Reactor Coolant System and the steam system is determined. The reactor coolant pumps are conservatively assumed to maintain full flow during the cooldown transient, resulting in the greatest rate of heat transfer to the turbine cycle, and thus the most rapid cooldown of the Reactor Coolant System. The initial full power heat transfer coefficient from the Reactor Coolant System to the Turbine Cycle System also is assumed to exist during the transient. Once the pressurizer is emptied, the Reactor Coolant System pressure is determined analytically, using the temperature output from the analog study combined with a mass and volume balance in the system.

A core water density calculation is then made to determine the net reactivity. For the most pessimistic reactivity calculation, the end-of-life (most negative) moderator coefficient is used, and the variation of coefficient with temperature is included. Minimum shutdown reactivity in rods is assumed, with the highest worth rod remaining fully withdrawn after reactor trip.

The analysis incorporates a time delay for the injection of borated water, consisting of the following:

a. Time required for cooldown to the point where the requisite safety injection trip conditions are reached

b. Response time for instrumentation to initiate the safety injection signa1

c. Time for operation of valves, starting of pumps, and the sequencing of interlocks in the Safety Injection System

d. Transport delay for the flow of borated water through the intervening pipe from the Safety Injection System to the reactor core

The rate of injection of borated water is determined as a function of the reactor coolant pressure according to the injection system head-flow characteristics. The injected water is assumed to mix with the reactor coolant flow past the inlet connection, and mixed boron concentration and enthalpy are determined. The boron concentration of the coolant in the core increases at intervals corresponding to the transit time of the coolant loop as the incoming safety injection water mixes with the previously borated loop flow.

In accordance with the design philosophy of the Safety Injection System as applied to loss of coolant protection, safety criteria are met if any single malfunction occurs in conjunction with the steam break. Consistent with 


\subsubsection{ASSUMPTIONS AND METHOD OF ANALYSIS (Continued)}

this philosophy, protection is evaluated on the conservative assumption that only one of the two trains of the Safety Injection System will operate.

Another single malfunction case evaluated is one which could result in the injection of unborated water into the Reactor Coolant system. As described in Paragraph 5.1, Safety Injection System, interlocks are provided on each feedwater train to assure that

a. Borated water has been flushed through the feedwater pump and its suction and discharge piping

b. Condensate isolation valves have completely closed before the safety injection header isolation valves can start to open

Furthermore, the condensate and heater drain pumps, which normally deliver unborated water to the feedwater pump suction, are automatically stopped by the safety injection signal. With this arrangement, the most adverse case of single malfunction causes injection of only that quantity of unborated water (approximately 23 cubic feet) initially contained between the feedwater pump suction and the discharge isolation valve in each of the safety injection trains. In this case, the reactivity effect of this malfunction is shown to be within acceptable limits, considering that the unborated flow is mixed with the borated water pumped by the other safety injection branch, and is preceded into the Reactor Coolant System by approximately six times its own volume of fully borated safety injection water which is always maintained in the delivery pipes during reactor operation. The Safety Injection System then continues to supply fully borated refueling water.

If, in a particular case, the cooldown results in a temporary return to criticality, the equilibrium power level reached is conservatively determined from an estimate of the minimum (absolute value) power coefficient. The rate of reactivity addition due to cooldown is slow enough to permit calculation of power on an equilibrium reactivity basis, i.e., there is no rapid nuclear excursion as a result of a return to critical.

The degree of possible core damage is calculated, assuming DNB to occur over the entire core. A heat transfer coefficient for film boiling is calculated and used, together with the stuck rod power distribution and calculated equilibrium power level, to determine cladding temperatures. The calculated cladding temperature would cause internal gas pressure to burst the rod. The percentage of rods exceeding this clad temperature is then calculated.

\subsubsection{RESULTS}

\section{General}

The maximum initial steam flow is calculated for a postulated break in the main 24-inch pipe. The break is assumed to occur at the tee connecting the steam generator for Loop $B$, from which the flow splits to the ring supply header. Flow is assumed to occur from all three connections to the tee. 


\subsubsection{RESULTS (Continued)}

The total flow through the break is directly proportional to the initial steam generator pressure. Since the steam pressure increases with decreasing load, maximum initial steam flow through the break exists at low power. For this break during full power operation, the steam flow from the steam generators would suddenly increase to approximately 400 percent of full load flow, including 100 percent flow continuing to the turbine until the reactor and turbine trips occur. From the no-load condition at a higher initial steam pressure (923 psia), the steam flow would suddenly increase to 385 percent of full load steam flow, with no flow to the turbine. This initial steam flow, without the compensating effect of a reduction of flow with turbine trip, results in a more rapid reactor coolant cooldown rate. The no-load condition is therefore the most severe steam break condition.

Figures 10.13 and 10.14 show the reactor coolant and steam generator temperatures as a function of time after the break for the no-load condition. The total steam flow from the steam generators is also shown which decreases as the steam generator pressure decreases. Figure 10.15 shows the pressurizer pressure and water volume versus time. The Safety Injection System is actuated on a coincidence of low pressurizer pressure and low pressurizer leve1 14 seconds after the break. For the no load case the feedwater pumps are assumed to be in a standby condition and a starting delay of 10 seconds is assumed to bring the feedwater pump up to speed. A seven-second delay is also assumed for the operation of the safety injection valves admitting safety injection water to the Reactor Coolant system. The total delay between actuation and the time safety injection water reaches the reactor coolant cold leg is 17 seconds. An additional transport delay of three seconds is assumed before the borated water reaches the core.

Figure 10.16 shows the reactivity insertion in the core resulting from the cooldown followed by safety injection. The core is assumed to be just critical at the initiation of the transient. The increase in reactivity is calculated for the most negative moderator coefficient, which occurs at the end of core life when the Reactor Coolant System is essentially boron free. The decrease in reactivity at 16 seconds is a result of a reactor trip actuated by the safety injection signal. The assumed shutdown is 2.2 percent $\delta k$ based on the end of 1 ife value assuming the highest worth rod stuck out and including margin for uncertainties as described in Paragraph 3.4, Reactor Nuclear Design. The reactivity continues to increase with continued cooldown until the borated water from the safety injection reaches the core at about 34 seconds. This figure shows the effect with one safety injection train and feedwater interlocks assumed to work. The small increase in reactivity at about 42 seconds is the effect of the 3.4 cubic feet of clean water which cannot be swept out of the system. The second rapid decrease in reactivity occurs at 45 seconds and is the result of the transport of borated water around the loop which then mixes with the safety injection stream essentially doubling the boron concentration. The continuum of this curve follows the same general trend. For this case there is a short term power increase to less than 50 percent power, and following reactor trip the core is maintained in a subcritical condition. If both safety injection trains function, the transient up to 34 seconds is the same and the reduction resulting from safety injection will be essentially twice the value shown and similarly at 45 seconds. 


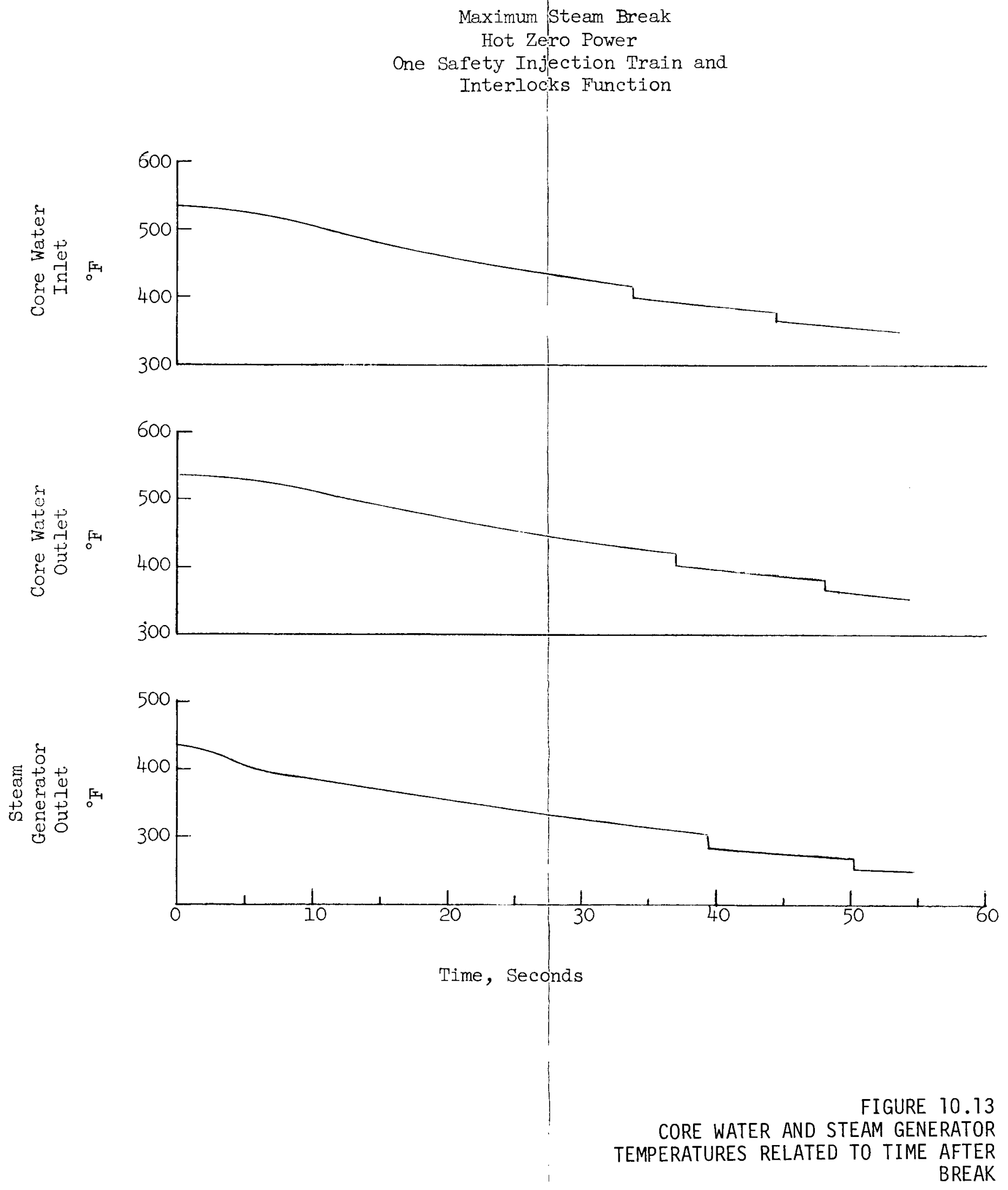




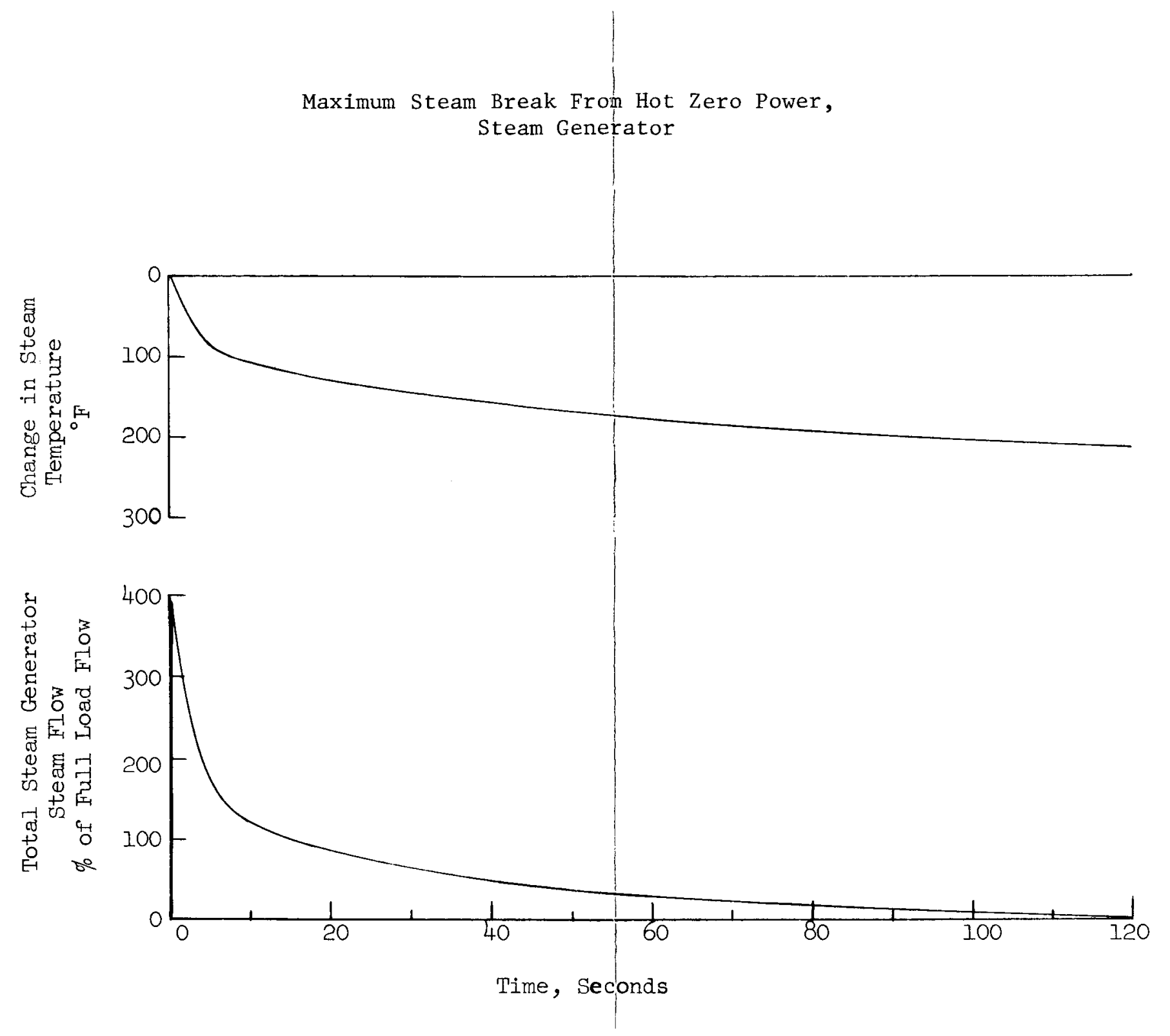

FIGURE 10.14

STEAM GENERATOR FLOW AND TEMPERATURES RELATED TO TIME

AFTER BREAK 

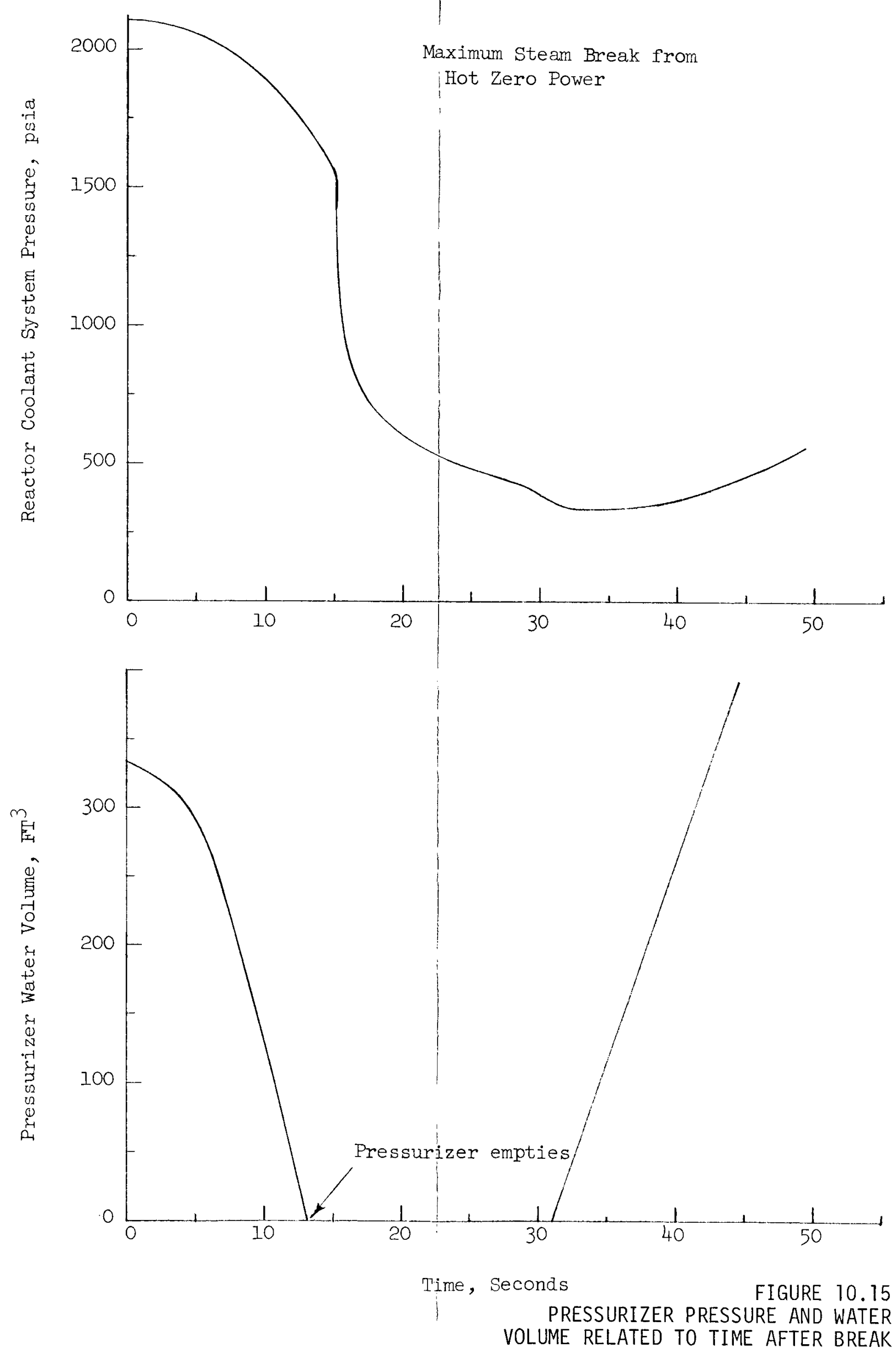


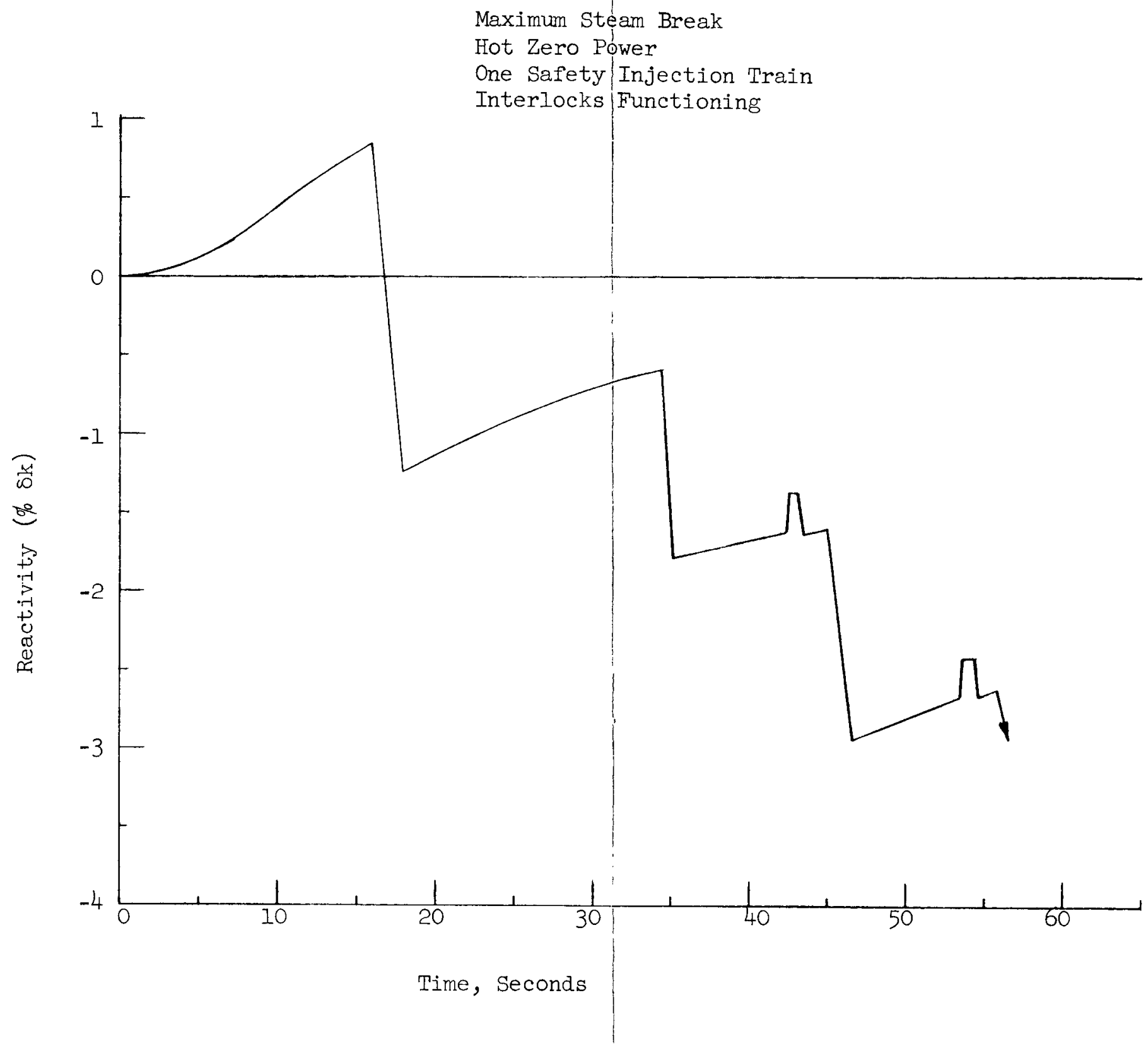

FIGURE 10.16 


\subsubsection{RESULTS (Continued)}

Several combinations of interlock failures were investigated for the no load steam break transient. Figure 10.17 shows the reactivity transient for one safety injection train assuming the valve interlock fails leading to the addition of approximately 23 cubic feet of clean water after an initial injection of 105 cubic feet of borated water at 2,900 parts per million. The clean water addition is limited by the automatic condensate pump trip. Since the valve interlock is assumed to fail, the borated safety injection water reaches the core seven seconds earlier. The short-term spike in reactivity at 35 seconds results from the unborated water addition from safety injection mixing with the original unborated reactor coolant prior to a complete loop transport of the borated water. No credit is taken for mixing in the core inlet plenum. Figure 10.18 shows other combinations of failures, none of which results in a return to criticality following the reactor trip. With both safety injection trains operating, and even if pump speed interlocks were assumed to fail, the unborated water addition effect is attenuated because the piping of two trains is not symmetrical in regard to the initial borated water addition or to the subsequent unborated water addition. There is, therefore, a mixing of borated water in one train with the unborated water from the other train.

\section{Hypothetical Injection of Unborated Water}

In order to estimate what limits may exist in the addition of unborated water during a steam break, a hypothetical calculation was performed. A shutdown of 1 percent $k$ instead of the calculated 2.2 percent $k$ was assumed. Both trains of the Safety Injection System were assumed to inject unborated water for an assumed period of time after the initial borated water is swept out of the injection lines. The resulting reactivity and power transients were calculated using the CHIC-KIN code (J. A. Redfield, "CHIC-KIN" - A Fortran Program for Intermediate and Fast Transients in a Water Moderated Reactor," WAPD-TM-479, January 1965) including void reactivity shutdown effects. The inputs to the code as a function of time were core inlet temperature, system pressure, reactivity from control rods following reactor trip and boron concentration changes resulting from safety injection. The external reactivity addition is shown in Figure 10.19 where the effect of reactor trip and boron concentration changes from safety injection are shown. The reactivity effects of Doppler and moderator are computed by the code.

The reactor trip is assumed to be effective at 16 seconds. Borated water reaches the core at 34 seconds followed by unborated water at 42 seconds. The effect of the loop transport delay is seen in the reactivity decrease at 34 seconds followed by another decrease at 45 seconds. The resultant heat flux transient is shown in Figure 10.20. The effect of the peak heat flux was analyzed assuming the most adverse power distribution with a control rod stuck in the fully withdrawn position. The corresponding radial power distribution is shown in Figure 10.21 with hot channel factor given as a function of number of rods exceeding the factor.

A conservative estimate of core damage resulting from this hypothetical case is obtained by assuming DNB to occur and by determining the resultant 


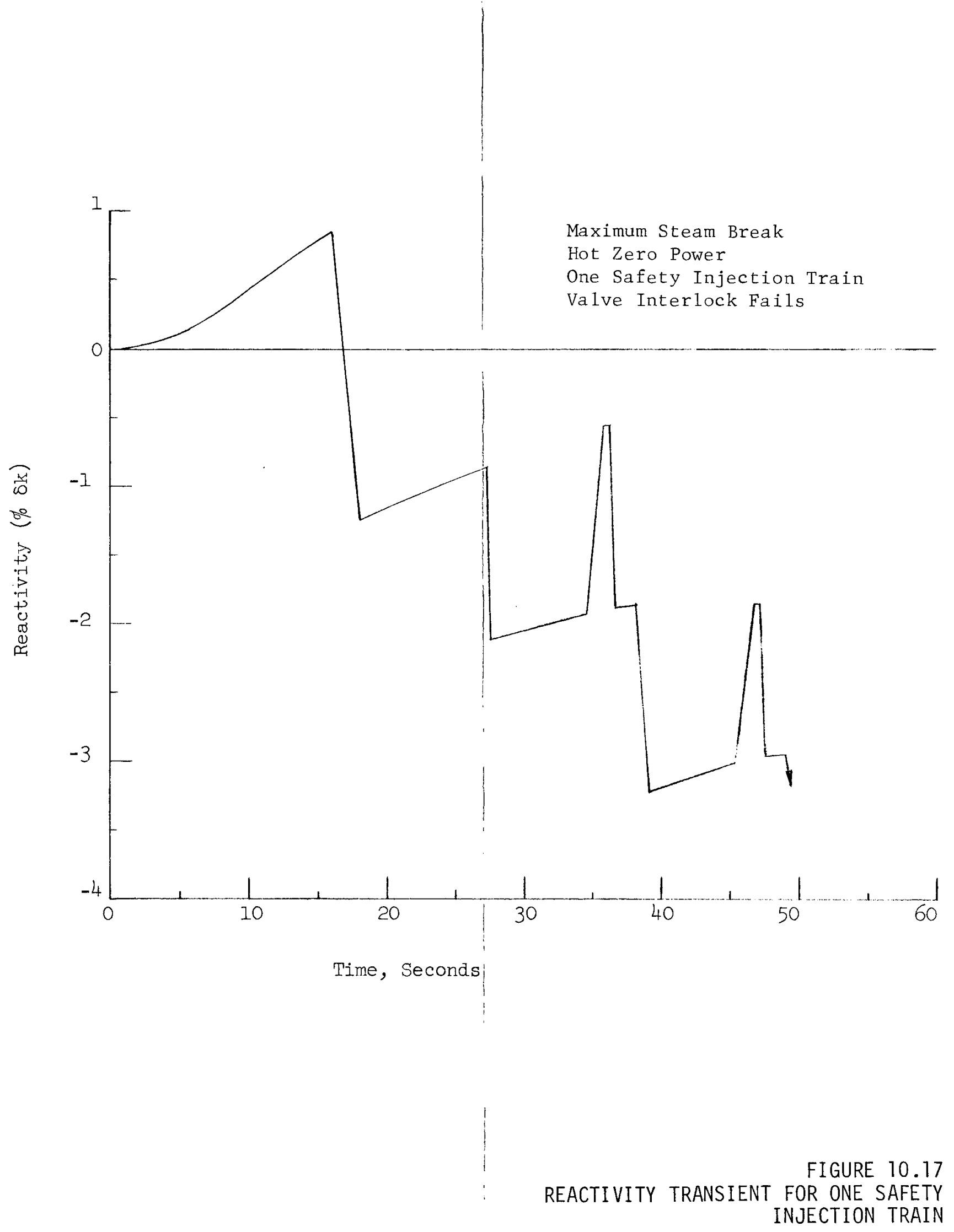




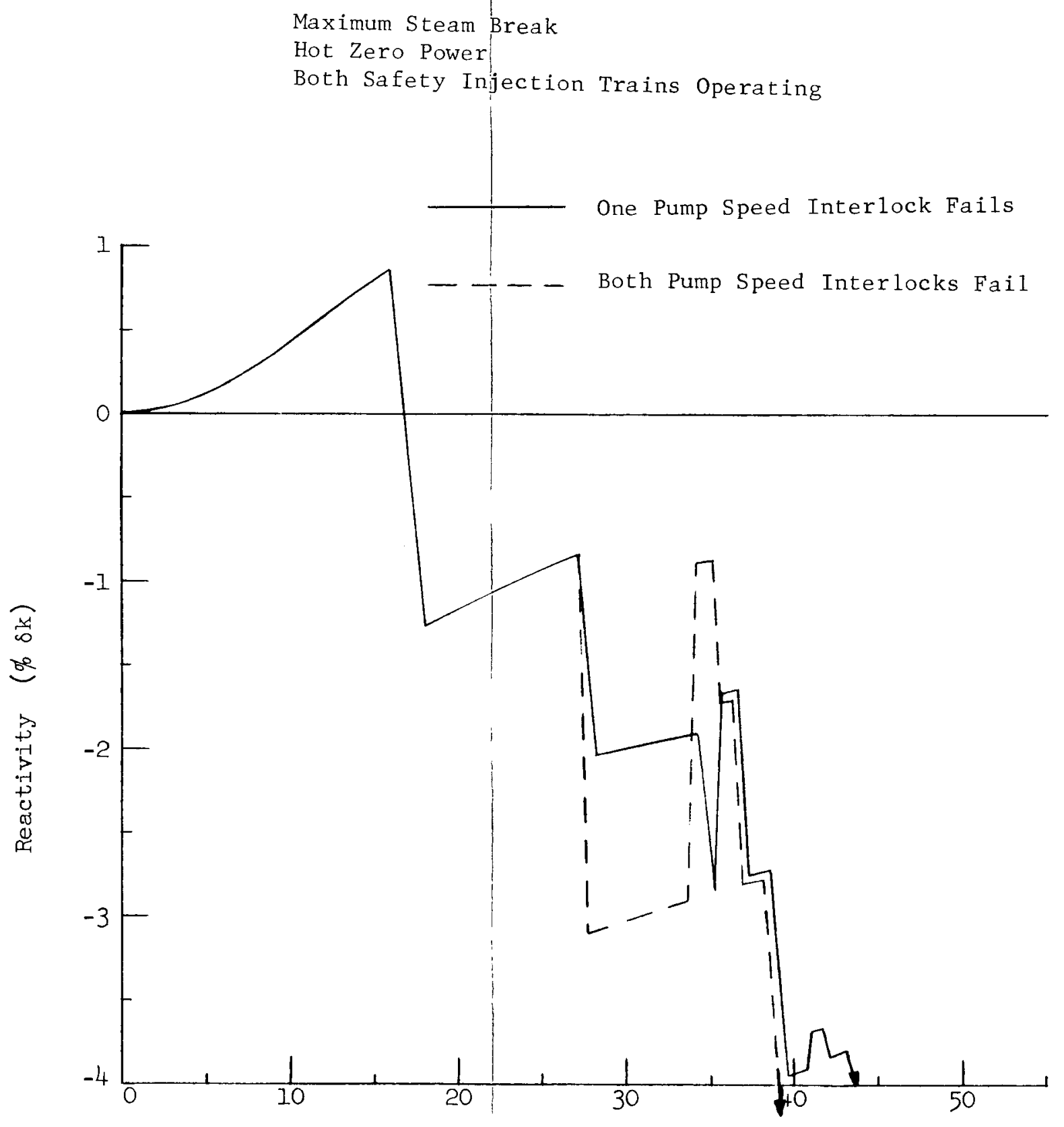

Time, Seconds

FIGURE 10.18 


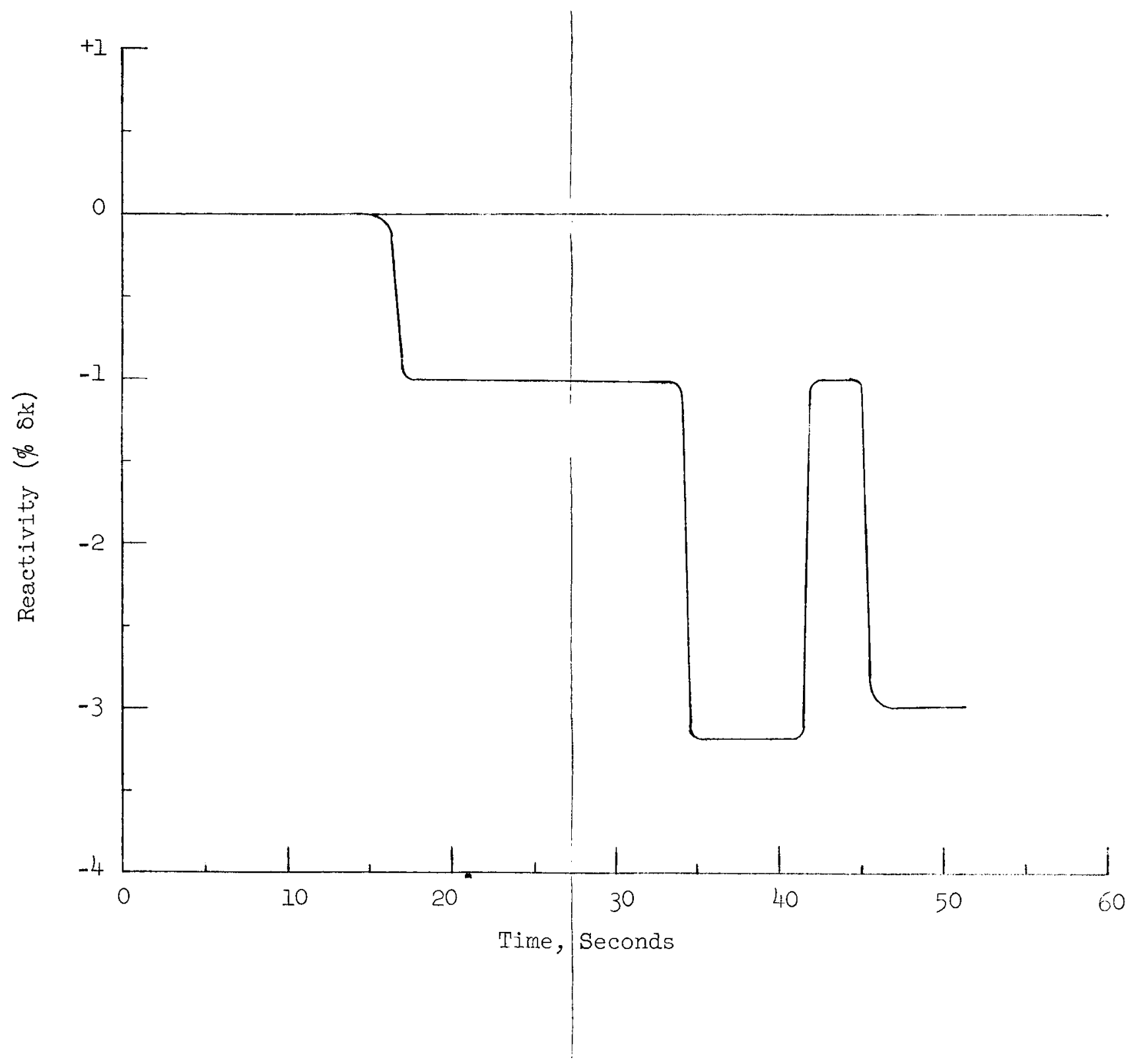

FIGURE 10.19 REACTIVITY INPUT TO CHIC-KIN CODE FOR "HYPOTHETICAL" CLEAN WATER ADDITION DURING STEAM BREAK 


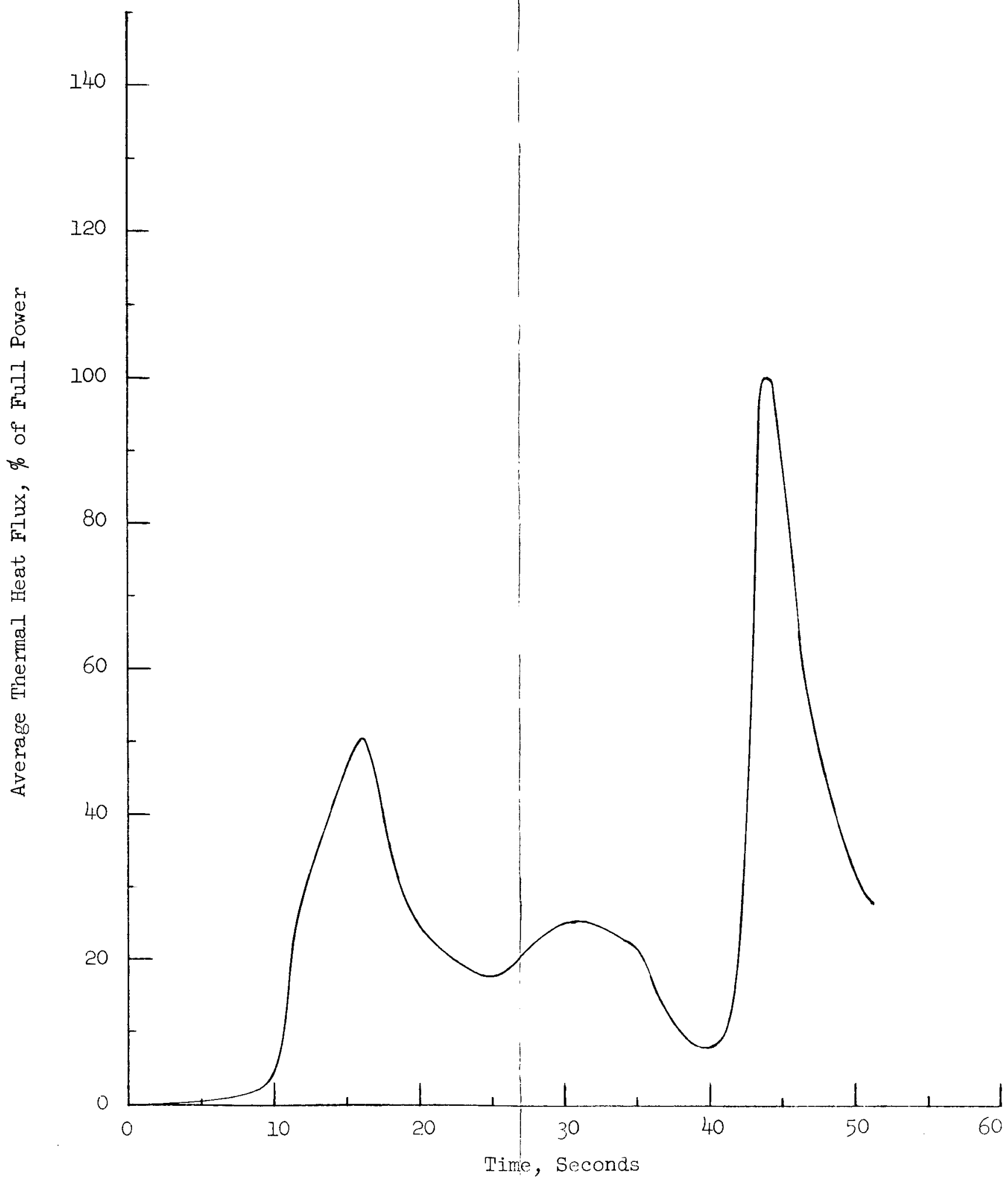

FIGURE 10.20

MAXIMUM STEAM BREAK "HYPOTHETICAL" CLEAN WATER ADDITION WITH ONE PERCENT SHUTDOWN FROM HOT ZERO POWER 


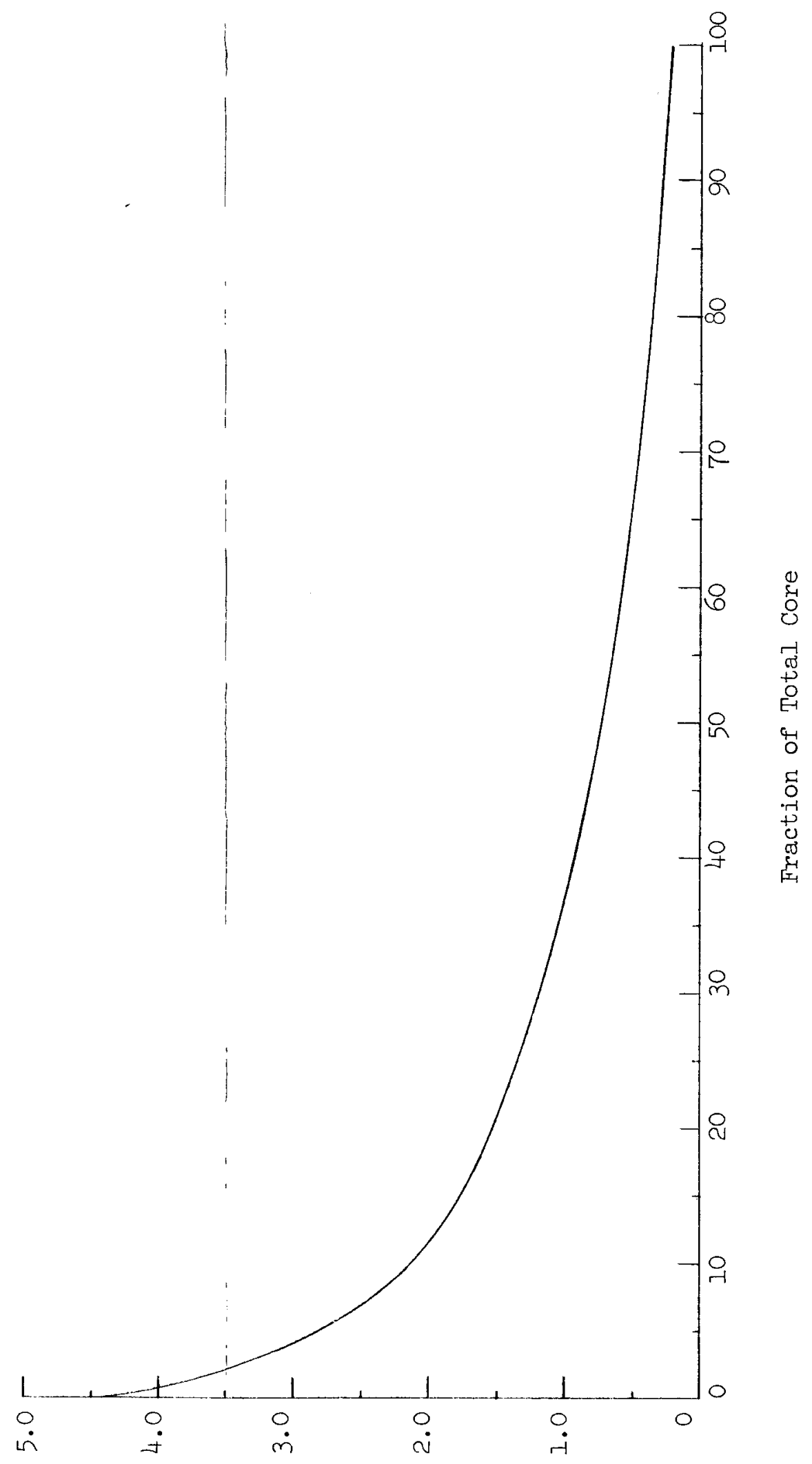

Nuclear Radial Hot Channel Factor

FIGURE 10.21 RADIAL POWER DISTRIBUTION WITH ONE STUCK ROD 


\subsubsection{RESULTS (Continued)}

cladding temperatures as a function of the power distribution. A conservative low film coefficient is calculated for the low pressure condition in the core; the value used was $600 \mathrm{Btu}$ per hour per square foot per F. A cladding temperature of 1,300 degrees Fahrenheit was calculated as a conservative upper limit for possible clad bursting. For these conditions, the peak heat flux at 44 seconds would yield no bursting. The total amount of unborated water that would be inserted into the reactor coolant up to about 52 seconds is approximately 450 cubic feet. After 52 seconds it is assumed the injected water is borated. Note that this is a hypothetical case with only one percent shutdown assumed and a total unborated water addition of about 450 cubic feet. The reactivity transient with the available 2.2 percent shutdown is shown in Figure 10.22 which shows there is no return to criticality after the reactor trip. These considerations show that there is no severe consequence in adding as much as 450 cubic feet of unborated water during the steam break even under conservative assumptions for shutdown margin.

Figures $10.23,10.24$ and 10.25 show the transients for the maximum steam break flow from initial operation at full power. The reactor is tripped at about five seconds from the overpower trip. There is an overshoot in nuclear power but no approach to DNB since the thermal flux is lagging and the coolant temperatures are decreasing. The reactivity transient is shown for 2.2 percent shutdown, and there is no return to criticality. One safety injection train is assumed to work and all interlocks are assumed to function. The initial cooldown rate for the full load case is less than that for the no load case since the change in steam flow through is less. There is a sudden reduction in steam generator steam flow at 6 seconds when the turbine is tripped.

The effect of an even smaller initial steam. flow is shown in Figure 10.26. The initial flow for this case is equivalent to a sudden opening of all steam dump valves yielding a sudden increase of 40 percent of full load steam flow from the no load condition. With this smaller additional flow the pressurizer pressure and level do not reach the safety injection trip point until about 60 seconds. The net reactivity insertion at this time is essentially the same as that introduced at safety injection trip point for the most severe break. The subsequent insertion rate due to cooldown is slower, however, for the smaller steam flow, and there is no return to criticality. The assumption made for this case was that both safety injection trains are working but both speed interlocks fail leading to the addition of about 45 cubic feet of unborated water.

\section{4 .5 CONCLUSIONS}

The effect of uncontrolled heat extraction from the Reactor Coolant System resulting from a steam supply system rupture was analyzed. For the break resulting in the maximum steam flow the Safety Injection System together with the shutdown available in rods is sufficient to prevent core damage. This analysis was performed for the most severe conditions with respect to reactivity addition, i.e., end of core life with the most negative moderator coefficient of reactivity. 


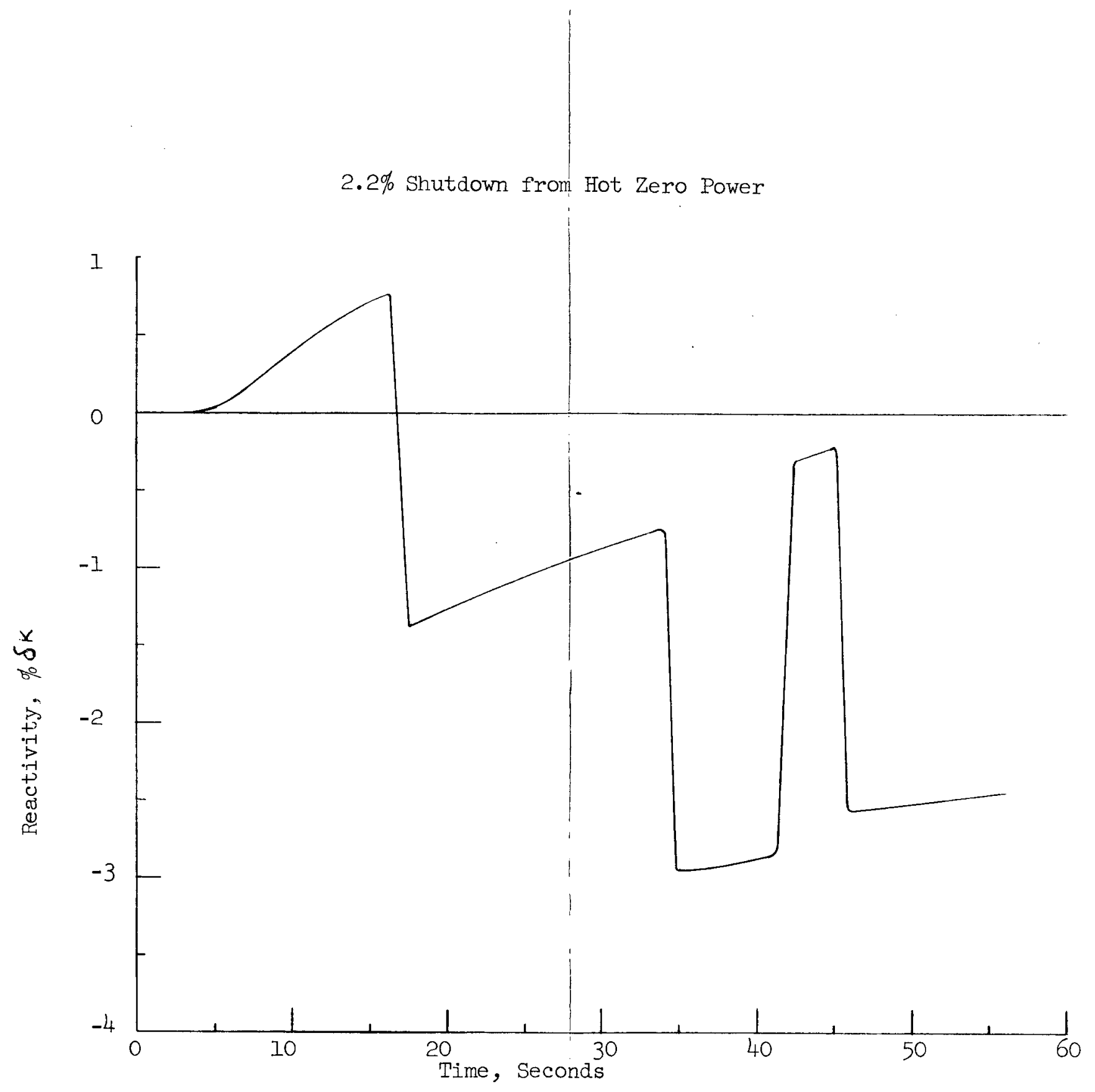

FIGURE 10.22

MAXIMUM STEAM BREAK WITH "HYPOTHETICAL" CLEAN WATER ADDITION 


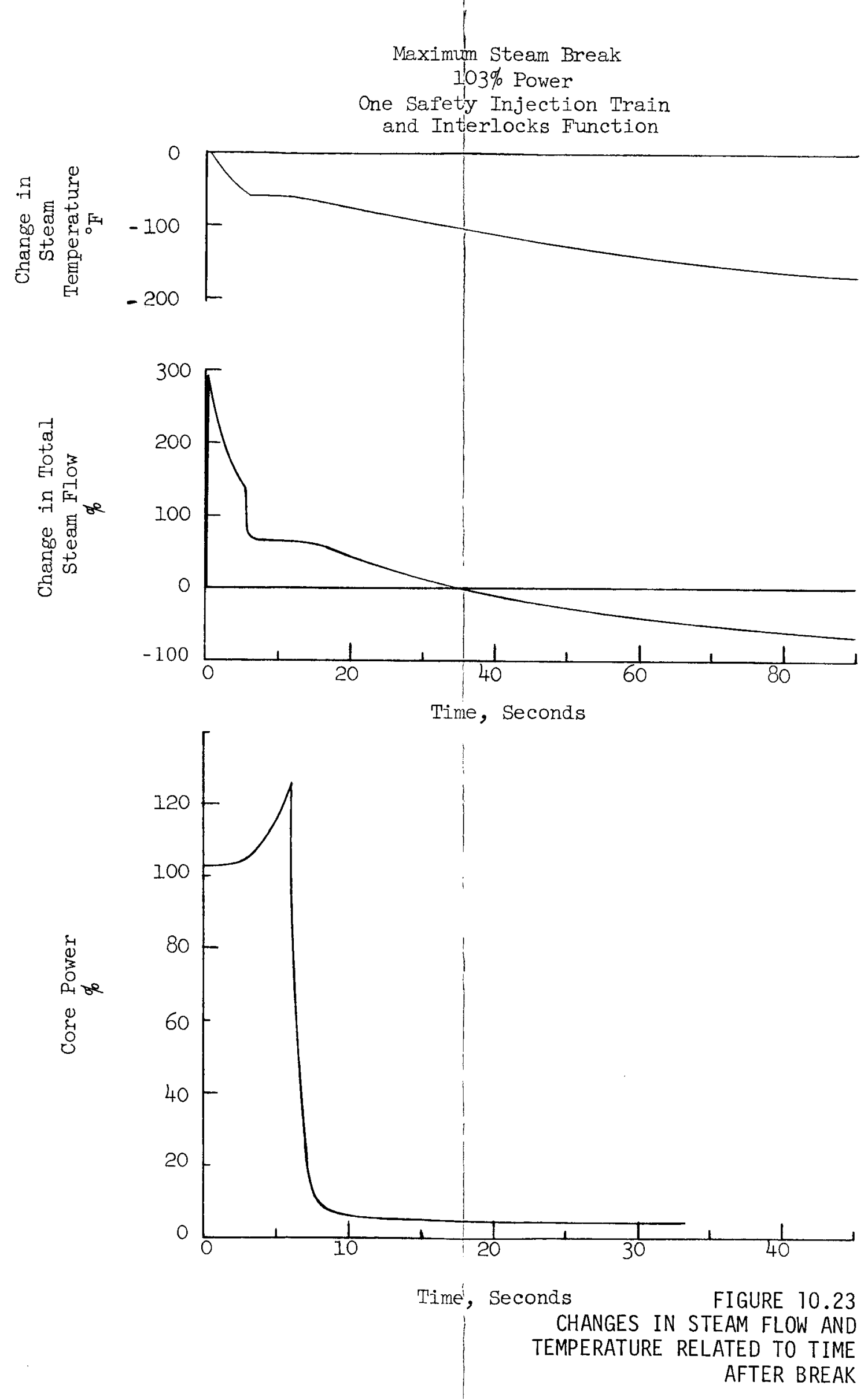



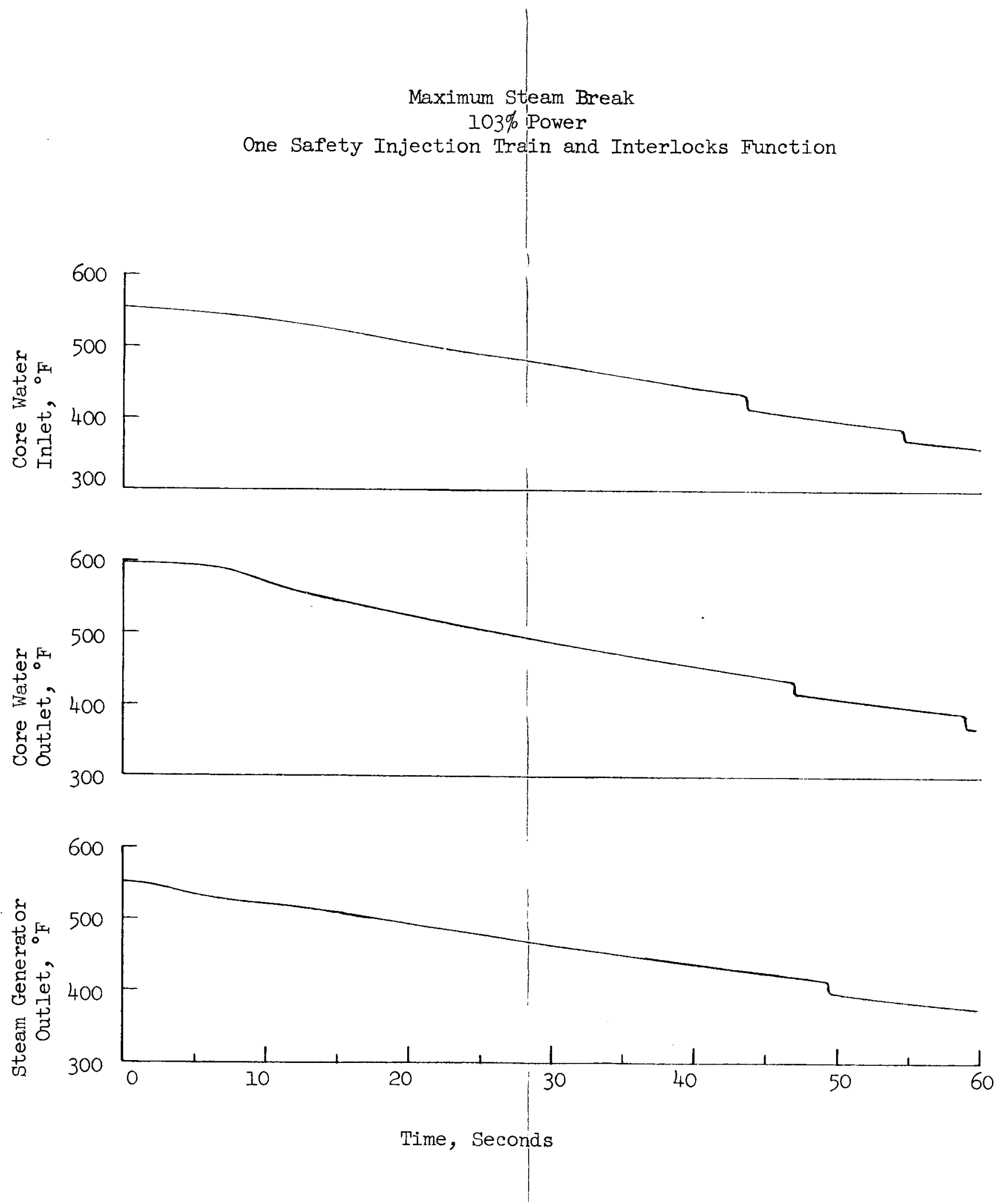

FIGURE 10.24 CORE WATER AND STEAM GENERATOR TEMPERATURES RELATED TO TIME AFTER BREAK 


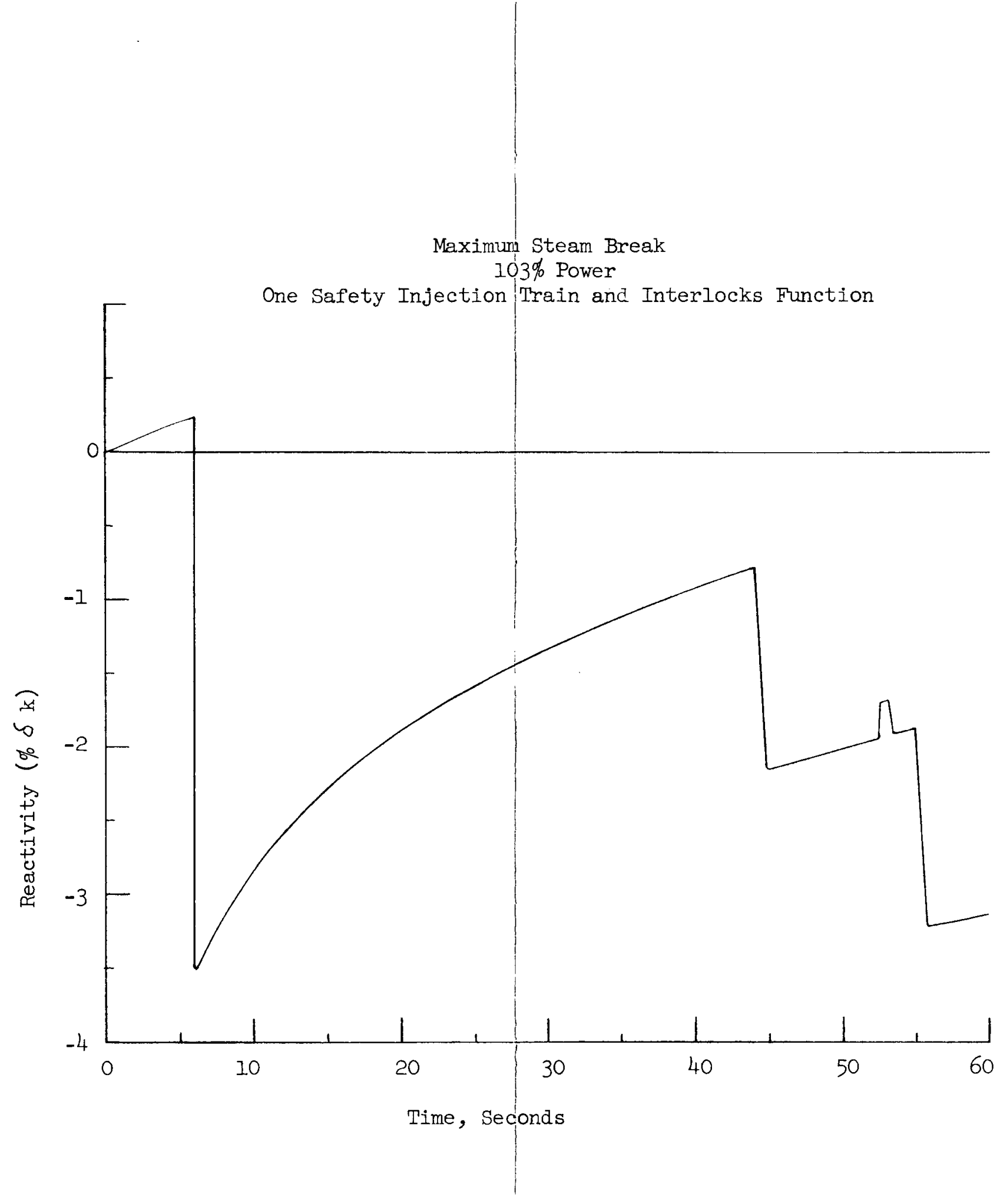

FIGURE 10.25 REACTIVITY RELATED TO TIME AFTER BREAK 


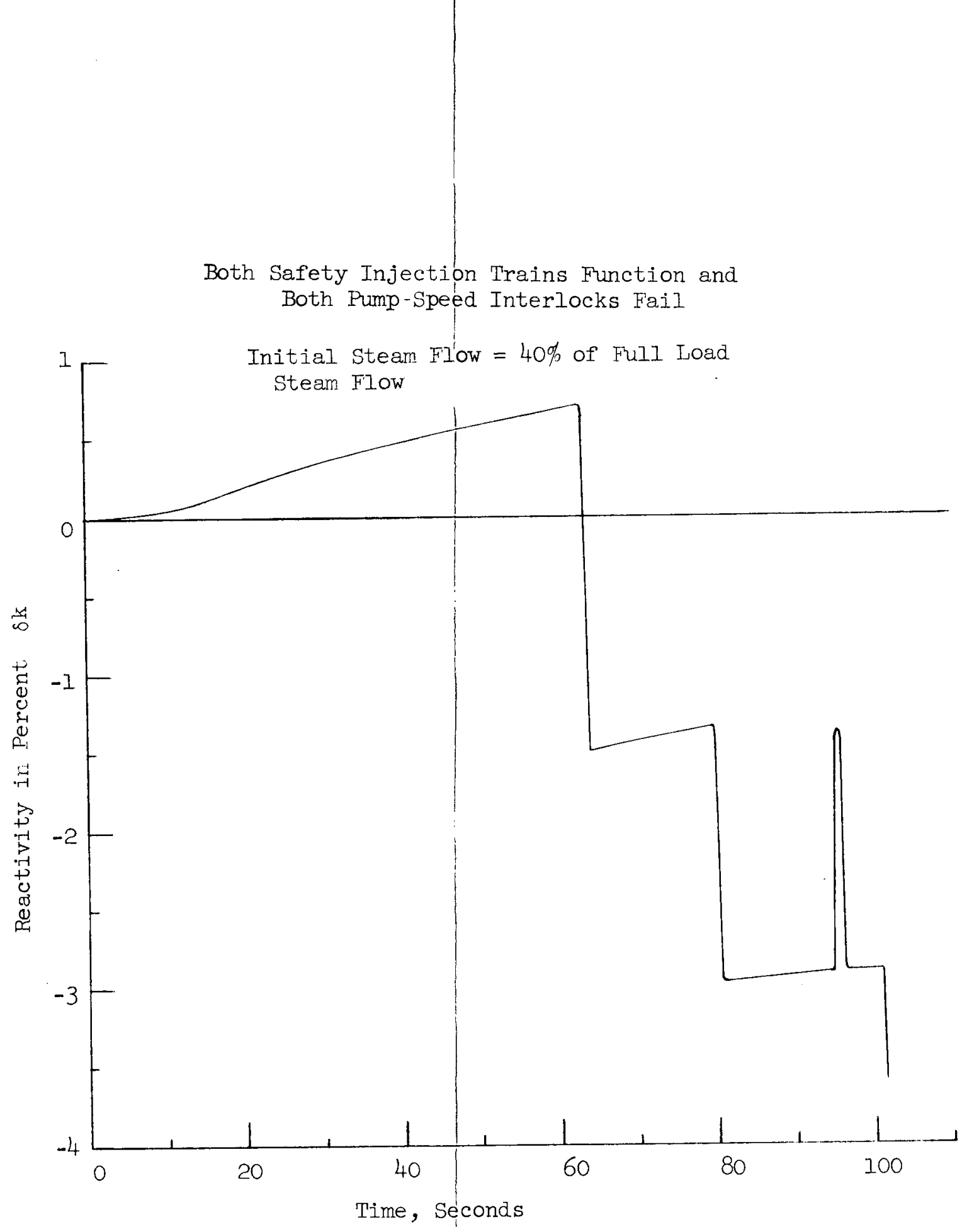

FIGURE 10.26

ACCIDENTAL OPENING OF ALL STEAM DUMP VALVES FROM NO LOAD

CONDITION 


\subsubsection{CONCLUSIONS (Continued)}

Several cases of assumed Safety Injection System interlock failures were investigated leading to various amounts of clean water addition. These analyses showed that in all cases a single interlock and in most cases multiple interlock failures can be tolerated during the steam break transient without adverse effects. A hypothetical case of reduced shutdown margin was studied with uncontrolled clean water addition following the initial injection of borated water contained in the safety injection piping. The results showed that even under these circumstances, the injection of 450 cubic feet of unborated water resulted in no core damage. 


\subsection{STEAM GENERATOR TUBE RUPTURE}

\subsubsection{GENERAL}

The integrity of the steam generator reactor to turbine cycle barrier is of significance from a radiological safety point of view because it prevents the transport of contaminants from the Reactor Coolant System to the turbine cycle. Should penetration occur, the steam piping, turbine, condenser, condensate, and feedwater systems would confine the non-gaseous material that might be carried by the steam. Gaseous activity, however, would be released through the air ejectors to the plant stack.

A complete severance of a steam generator tube has been analyzed to show the effectiveness of plant operating limits (such as maximum permissible coolant activity) and emergency shutdown procedures in restricting the stack release to safe levels, in this limited case of tube rupture.

Buildup of non-gaseous activity in the steam generator shell-side liquid following tube rupture is not of immediate significance, because there is no direct path for the release of this activity. This liquid is sampled as a safeguard against improperly discharging blowdown wastes containing reactor coolant activities above permissible levels.

Because the steam generator tube rupture results in a loss of reactor coolant, it imposes a requirement on supplemental coolant makeup, by charging flow and possibly safety injection, to prevent pump cavitation and eventual uncovering of the core. Protection against a loss of coolant of the magnitude of a tube rupture was analyzed in the category of small breaks in Paragraph 10.1. That analysis shows that there would be no release of fission products to the turbine cycle other than those initially present in the reactor coolant prior to the break.

\subsubsection{FACILITY DESTGN ASPECTS}

In the design and manufacture of the steam generator, prevention of tube rupture or separation at the tube sheet joint is implemented by:

- rigid quality control of the Inconel tubing material

- conformance to Section VIII of the ASME Boiler and Pressure Vessel Code, and

- hydrostatic testing at a pressure differential between tube and she11 of 2.3 times that which exists in normal service.

Crevice corrosion tests have been performed under conditions much more severe than would be expected in the plant. The tests showed that Inconel is highly resistant to this type of attack.

Tube support plates are designed to withstand the hydraulic forces associated with five times full rated steam flow without structural damage. 


\subsubsection{FACILITY DESIGN ASPECTS (Continued)}

A rupture or pullout failure of a steam generator tube has never occurred in a unit designed to these or equivalent standards, despite several hundred unit-years of service.

Should such a failure occur, however, the following plant features and procedures would be employed to limit the consequences:

a) The air ejector radiation alarm would be actuated about 16 seconds after rupture by the appearance of Nitrogen-16, a shortlived reactor coolant activation product. Proper functioning of the radiation monitor would be verified, and then the reactor would be manually tripped. Other indications of tube rupture would include: stack radiation alarm; steam generator blowdown sample radiation alarm; loss of normal level in pressurizer; and volume control tank alarms.

b) Maximum available reactor coolant makeup capacity is brought into operation automatically as a result of falling water level in the pressurizer. This automatic action includes starting the standby charging pump. If these measures are inadequate to balance the rate of coolant loss, the Safety Injection System is automatically actuated. In either case, sufficient supplementary water is added from the refueling water storage tank to prevent uncovering the top of the core. The same condition which initiates safety injection would cause an automatic reactor trip if manual trip had not been executed.

c) To minimize the discharge of reactor coolant through the ruptured tube, the pressure differential across the break would be reduced by manually initiating cooldown and depressurization of the Reactor Coolant System. In this procedure, boron concentration is brought to the cold shutdown level or greater by the addition of refueling water through the charging pumps, and/or safety injection. Shell-side pressure is manually regulated by operating the steam bypass valves which direct steam to the condenser; primary pressure is regulated by manually operating the pressurizer spray valves.

d) Cooldown is accomplished under manual control at a rate not exceeding the temperature transient rate established for reactor vessel thermal stress limitation (see Paragraph 3.5). When the appropriate pressure and temperature are reached, the residual heat removal loop is placed in operation. Further requirement for steam bypass having been eliminated, the reactor coolant pumps are stopped, the steam bypass valves are kept closed, and any additional leakage through the break is confined to the steam generator.

\subsubsection{ASSUMPTIONS AND METHOD OF ANALYSIS}

The consequences of a steam generator tube rupture can be analyzed by determining the maximum reactor coolant discharge rate for reactor and turbine cycle pressure conditions, as well as temperature conditions encountered during the recovery procedure outlined above. The net effect of discharge and makeup from available sources is integrated over the term of the recovery. 


\subsubsection{ASSUMPTIONS AND METHOD OF ANALYSIS (Continued)}

To determine the maximum transfer of reactor coolant to the turbine cycle, it is conservatively assumed that the operator on duty will neglect to take action when radiation alarms first indicate the occurrence of a break. Instead, it is assumed that the initial action will be only by automatic control. Reserve charging flow would be initiated by low charging header pressure (the condition that arises when the pressurizer level control calls for more flow than the charging pump can deliver). The reactor trip and safety injection would be actuated when the requisite two-out-of-three low pressure and low pressurizer level signals occur.

After safety injection trip, it is assumed that cooldown and depressurization would be executed by manual control at the allowable rate, with the leak rate during this period based on a differential pressure of 200 psi across the ruptured tube. A net positive pressure differential of about 200 psi is maintained to prevent cavitation of the reactor coolant pumps. Therefore, it would not be practical to eliminate the driving force for leakage across the break during this phase.

Because no cladding rupture would occur, the radiological consequences can be conservatively assessed by assuming that all of the radioactive material initially present in the reactor coolant would be transferred through the break in the course of the recovery period. This assumption is pessimistic because some of the initial coolant activity will remain in the Reactor Coolant System, diluted by the makeup water.

It is assumed that the gaseous fission products, zenon and krypton, would separate from the condensate without holdup in the turbine cycle. These gases would be carried to the plant stack through the air ejectors as long as it was necessary to maintain condenser vacuum. Vacuum would be maintained throughout the detection and depressurization phases, until the residual heat removal loop is placed in operation.

Non-volatile fission and corrosion products would largely concentrate in the feedwater of the faulty steam generator. A fraction of this activity would be carried over the moisture droplets to the steam piping, thence to the turbine moisture separators (prior to trip) and the condenser. During a brief interval after reactor trip, automatic pressure control in the steam system would actuate a steam dump to the atmosphere. The activity associated with moisture droplets (estimated at 0.25 percent of the steam mass flow) is assumed to be discharged as airborne particles in a groundlevel cloud. During the remainder of the recovery period, non-volatile activity in carryover would accumulate in the condenser liquid phase. Partition of the radio-iodine between the liquid and the non-condensibles leaving the condenser is estimated based on a decontamination factor of 10,000. It is assumed that the resulting vapor fraction would go to the stack by way of the air ejectors.

The magnitude of the radioactive discharges calculated in this manner are a measure of the worst-case consequences of a tube rupture for a given level of activity in the reactor coolant. The environmental consequences of such a release, presented in Section 15, show the relationship of normal and 


\subsubsection{ASSUMPTIONS AND METHOD OF ANALYSIS (Continued)}

maximum coolant activity to consequences considered limiting for public safety in this incident.

\subsubsection{RESULTS}

The analysis outlined above was performed for the hypothesis of a doubleended rupture of one steam generator tube, occurring while the plant is at full rated power with a maximum steam bypass requirement. The calculated fission product releases, based on a reference reactor coolant activity level corresponding to one percent defective fuel rods, were as follows:

a) During the period of direct steam bypass to the atmosphere, which lasts approximately 60 seconds, the release of entrained iodine activity as equivalent I-131 is calculated to be 0.005 curies. In evaluating the release of a mixture of iodine isotopes with respect to permissible limits under $10 \mathrm{CFR} 20$, each isotope can be treated as an equivalent amount of I-131, obtained by dividing the actual number of curies by the ratio:

Maximum Permissible Concentration of the isotope in question Maximum Permissible Concentration of I-131

A similar procedure is followed in the case of a noble gas mixture, using $\mathrm{Xe}-133$ as the reference activity.

Noble gas activity released during the same interval by way of the atmospheric bypass would be 850 curies as equivalent $\mathrm{Xe}-133$. This release would begin approximately two minutes after the occurrence of the tube rupture, corresponding to the time the reactor trip were initiated by the safety injection signal. If the reactor were manually tripped prior to this signal, the activity release would be less, due to the reduced accumulation of reactor coolant impurities in the feedwater at the time the steam bypass was opened.

b) Throughout the remainder of the depressurization procedure, noble gas and entrained soluble activities would be carried to the condenser by the intermittent steam flow required for cooldown and residual heat dissipation. The total carryover was calculated for the end-of-plant life case (longest cooldown, hence maximum integrated steam flow). The resulting release at the plant stack, integrated over the 4.3-hour cooldown period, was 26,443 curies equivalent Xe-133, and less than 0.031 curies equivalent I- 131 .

\subsubsection{CONCLUSIONS}

The result for other fuel defect conditions would be directly proportional to the assumed percentage of defective rods. The magnitude of the assumed release is compared with the acceptable limits in Paragraph 15.2, and is shown to consitiute no undue hazard to the public. 


\subsubsection{GENERAL}

A loss of load analysis is performed to determine the effects of a rapid and large reduction in generator load which would in turn cause a rapid and large reduction in the heat extracted from the Reactor Coolant system. If the decrease is greater than that which the system is designed to accept, with no protective action the resultant increase in reactor coolant temperature and pressure could exceed the normal core operating limits. To preserve the integrity of the cladding, the Reactor Control and Protection System initiates a reactor trip in time to prevent DNB. The trip will also limit the maximum pressure rise in the Reactor Coolant System; however, ultimate overpressure protection by the pressurizer safety valves is capable of limiting the Reactor Coolant System pressure to a value below the trip point, even for the maximum possible loss of load. Protection of these barriers is therefore independent.

\subsubsection{FACILITY DESIGN ASPECTS}

The station is designed on a conservative basis to accept, without reactor trip but with automatic steam release to the atmosphere and condenser, a 30 percent step reduction from 450-Mwe generator load and a 50-percent step reduction from 395 Mwe load. A 10-percent step reduction of 450-Mwe load would not require steam release.

The greater the magnitude of the sudden load decrease the more severe are the system transients. To prevent the possibility of clad damage in severe cases beyond 30-percent reduction from 450-Mwe load, the Reactor Control and Protection System is designed so that a reactor trip initiated by conditions exceeding either the variable low pressure set point, or in some cases, the high pressurizer pressure or high pressurizer level set points, wi11 prevent DNB. As described in Paragraph 6.2, Reactor Control and Protection System, each of these channels functions on a two-out-of-three coincidence principle.

The most likely source of a complete loss of load is a turbine generator trip. In this case there is a direct reactor trip signal derived from turbine autostop oil pressure (a two-out-of-three circuit) and there is no temperature or pressure increase in the Reactor Coolant System. The plant behavior is, however, evaluated for a complete loss of load from 450 Mwe without a reactor trip, primarily to show the adequacy of the pressure relieving devices, as wel1 as to show that no core damage occurs. The Reactor Coolant System and Turbine Cycle System pressure relieving capacities are designed for this condition.

A partial loss of load may result from turbine governor response to a sudden significant increase in frequency of the distribution network - a remote possibility in view of the electrical characteristics of the Southern California Edison Company and San Diego Gas \& Electric Company systems to which the San Onofre plant is connected. In this case there is an increase in reactor coolant and steam temperature, and system pressures will increase. 


\subsubsection{FACILITY DESIGN ASPECTS (Continued)}

The Reactor Control and Protection System and steam release to the atmosphere and condenser, if in automatic control, will respond to try to limit these excursions. If the load decrease is within their capability, equilibrium system conditions are restored at the reduced load without further incident. If the load decrease exceeds the capability of these systems, or if the systems are in manual control, a reactor trip will be initiated to protect the core. Automatic reactor control and/or steam dump are not required as a design basis for the safety of the plant.

\subsubsection{METHOD OF ANALYSIS AND SELECTION OF PARAMETERS}

The loss of load transient is analyzed as part of the complete and detailed plant analog simulation study. The objectives of this analysis are twofold: to determine margins to core protection limits, and to establish pressure relieving requirements for the reactor coolant and turbine cycle systems.

Conservative results for the loss of load transient are assured by the following assumptions:

a. Initial operating conditions - the initial power level, coolant temperatures and pressure are assumed to be at their maximum values consistent with steady-state operation, including allowances for calibration and instrument errors. This assumption results in the maximum power difference for the load loss, and the minimum margin to core protection limits at the initiation of the loss of load.

The initial conditions are:

$$
\begin{aligned}
& \text { Power }=(1.03)(1,347 \text { Mwt }) \\
& \mathrm{T}_{\text {in }}=553 \mathrm{~F}+4 \mathrm{~F}=557 \mathrm{~F} \\
& \text { Pressure }
\end{aligned}
$$

b. Moderator coefficient of reactivity - assumed to be the most positive value expected during core life, i.e., beginning of life, while operating at power; this results in the minimum power decrease during the transient, and may result in a power increase $\left(\alpha_{W}=+0.5 \times 10^{-4} \delta \mathrm{k} / \mathrm{F}\right)$.

c. Doppler coefficient of reactivity - assumed to be the smallest (absolute value) negative coefficient, again resulting in the maximum power during the transient $\left(\alpha_{\mathrm{f}}=-1.0 \times 10^{-5} \delta \mathrm{k} / \mathrm{F}\right)$.

d. Reactor control - the reactor is assumed to be in manual contro1; no control rods are assumed to be inserted, either manually or automatically, prior to the initiation of reactor trip.

e. Steam release - steam release to the atmosphere and condenser is assumed to be inoperative. 


\subsubsection{METHOD OF ANALYSIS AND SELECTION OF PARAMETERS (Continued)}

f. Pressurizer spray and power operated relief valves - no credit is assumed for the beneficial effect of spray and relief valves in reducing or limiting pressurizer pressure.

\subsubsection{RESULTS}

The transient response for a complete loss of load from 450-Mwe operation is shown in Figures 10.27 and 10.28. The assumptions for initial conditions and parameters are as given in Paragraph 10.6, Loss of Load. The results are primarily shown to indicate the effective capacity of plant pressure relieving devices without an immediate reactor trip and with a positive moderator coefficient. For this case, the reactor is assumed to continue at power until the high pressurizer water level trip is reached at 34 seconds. The reactor would actually be tripped immediately upon a turbine trip from a signal from low autostop oil pressure (two-out-of-three circuit) and there would be no temperature or pressure increase in the Reactor Coolant system. For the case assumed above, the maximum volume insurge before trip is conservatively estimated, considering an initial minimum pressurizer water level, including adverse instrument and control errors, and considering a maximum level trip point, again including adverse instrument errors. Even under these assumptions there is only a 25-cubic foot overshoot in volume surge. The maximum surge is therefore well below filling the 1,300-cubic foot pressurizer volume. An instrument delay of two seconds was assumed for trip actuation. The steam generator temperature (hence pressure) rises to the safety valve setpoint (nominal 1,000 psia) in about seven seconds where the safety valves have sufficient capacity to maintain pressure throughout the transient. The pressurizer pressure increases to the safety valve setpoint (nominal 2,500 psia) in 12 seconds, where the capacity is sufficient to maintain pressure.

The analysis of plant protection is based upon the fixed high pressure reactor trip actuated at 2,250 psia, which will limit the transient at approximately seven seconds and prevent DNB in the core. With the high pressure reactor trip the minimum DNB ratio achieved during the transient is 1.55 . Note the increase in reactor power due to the positive moderator coefficient. No control rod motion is assumed to occur before the reactor trip. The maximum overpower trip level with errors is 118 percent, with a minimum possible trip point of 103 percent. Thus the overpower could also result in a trip to limit the transient.

With a negative moderator coefficient of reactivity the transient is less severe since core power level will decrease before the trip, as shown in Figures 10.29 and 10.30. The conditions are the same as those in Figures 10.27 and 10.28, with the exception of the moderator coefficient equal, in this case to $-0.5 \times 10^{-4} \delta \mathrm{k}$ per degree Fahrenheit. The resulting transient shows a high pressure reactor trip at about eight seconds, and the minimum DNB ratio is 1.96. With the most adverse errors leading to the maximum volume insurge before the pressurizer high water level reactor trip ( 300 cubic feet), a level trip would not occur. For this case the level trip was assumed to occur at 250 cubic feet. Note that for this case, equilib- 


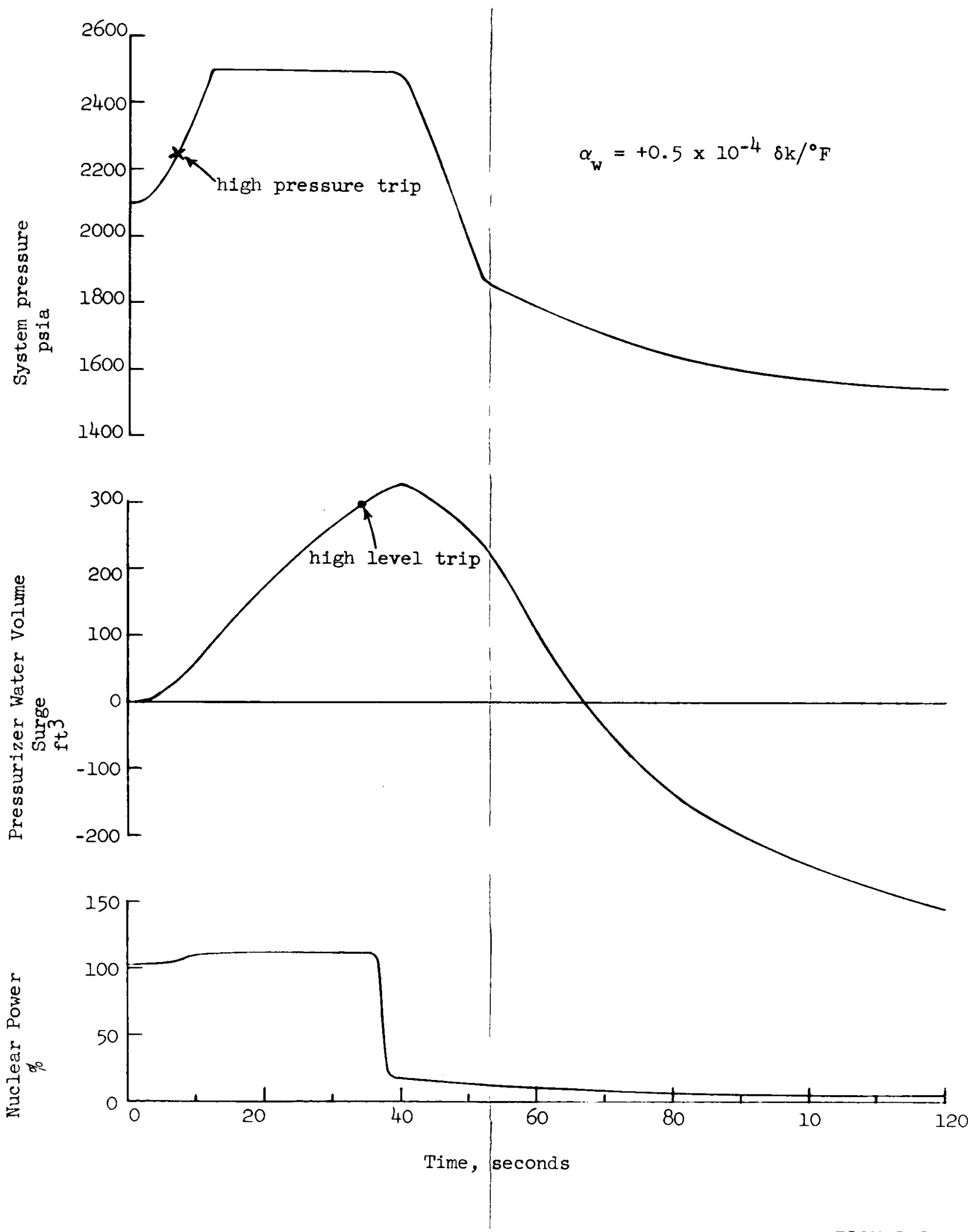

FIGURE 10.27

TRANSIENT RESPONSE FOR A FULL LOSS OF LOAD FROM 450 MWE POSITIVE

FACTOR 


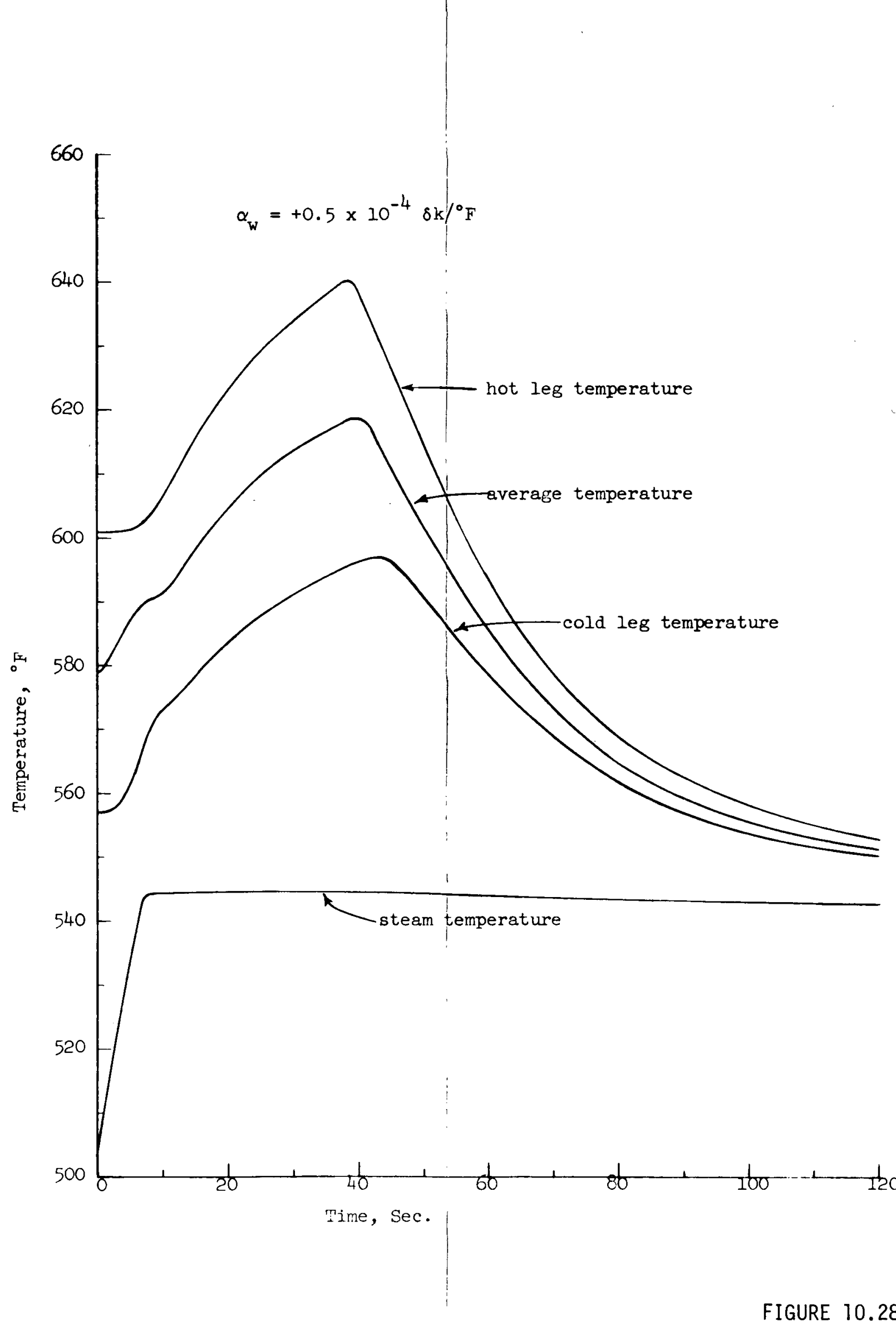

FIGURE 10.28

TRANSIENT RESPONSE TO A FULL LOSS OF LOAD FROM 450 MWE 

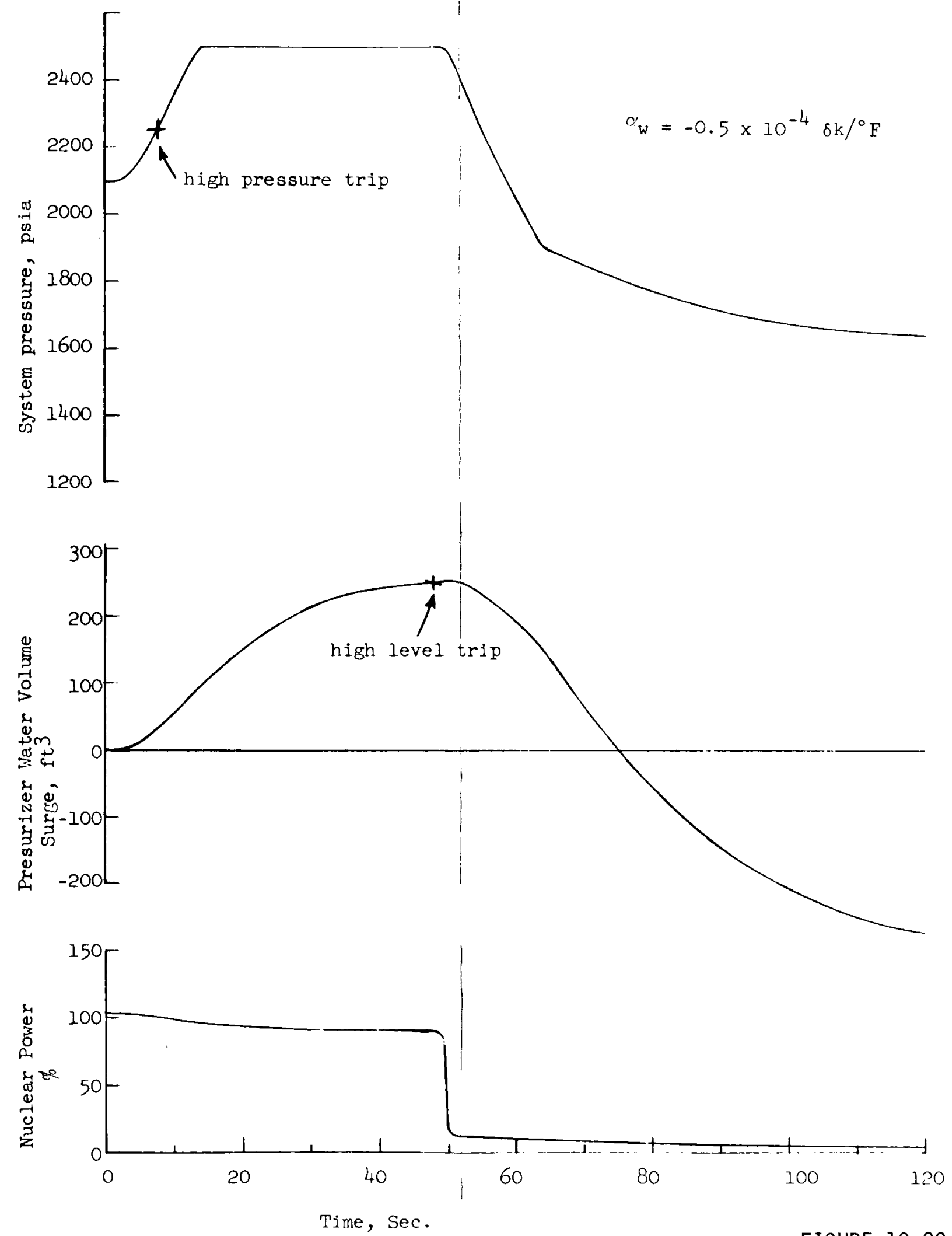

FIGURE 10.29

TRANSIENT RESPONSE FOR A FULL LOSS OF LOAD FROM 450 MWE NEGATIVE FACTOR 


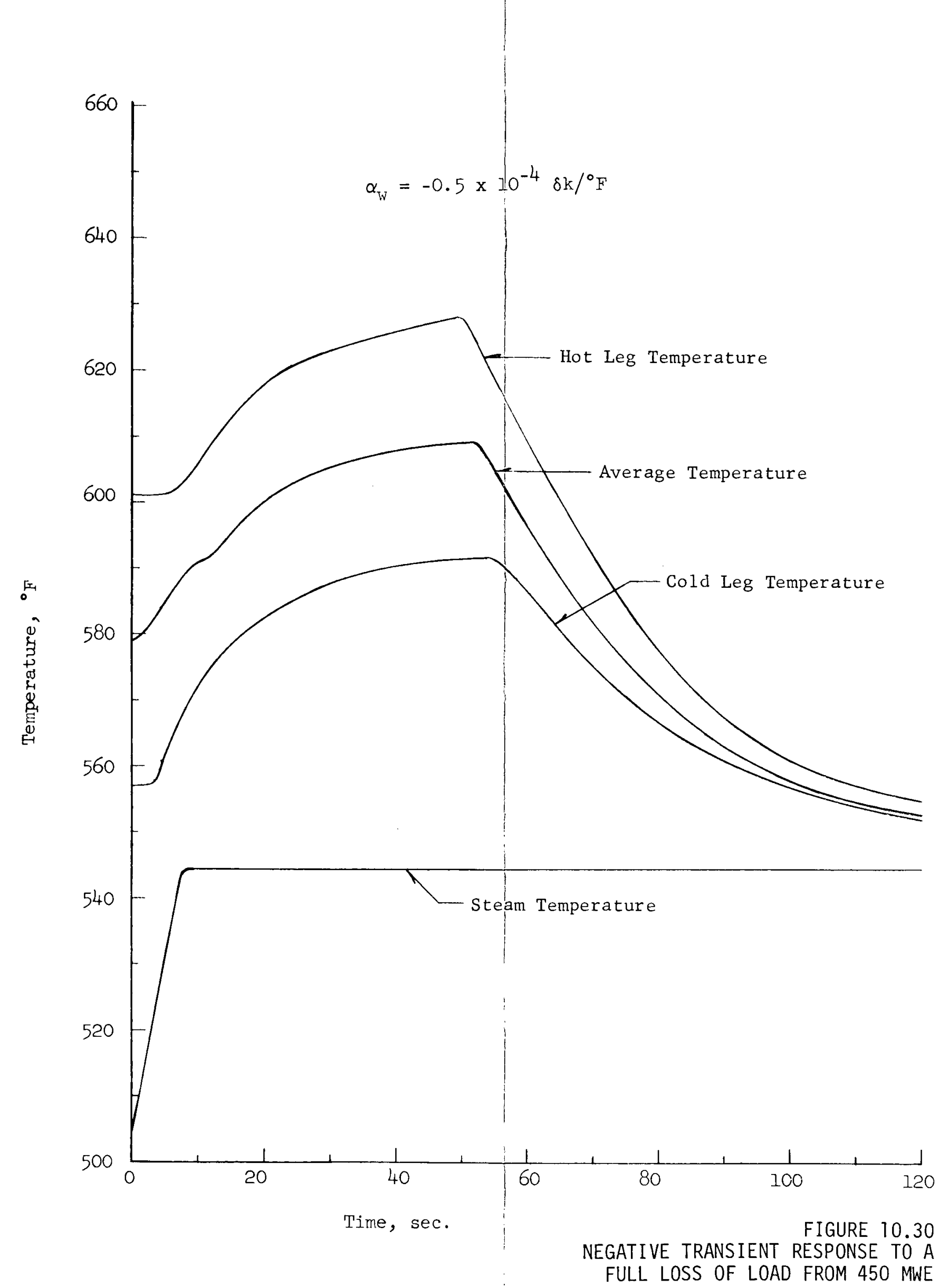




\subsubsection{RESULTS}

(Continued)

rium conditions are being approached, with a resultant very small rate of insurge to the pressurizer.

In actual operation the pressurizer pressure would be rapidly reduced to approximately 2,150 psia by the power operated relief valves and by pressurizer spray, which are both duplicated. The reduced pressure, in conjunction with the elevated reactor coolant temperatures, would result in a variable low pressure reactor trip, a further protective feature for the transient.

The minimum DNB ratio reached, when the high level trip is assumed, is 1.68 , showing that the fixed high pressure reactor trip is not necessary for DNB protection when a negative moderator coefficient exists, causing a core power reduction during the transient. The pessimistic combination of most positive moderator coefficient and least negative Doppler coefficient was selected for the transient shown in Figures 10.27 and 10.28

\section{$10 \cdot 6.5$ CONCLUSIONS}

The complete loss of load, without a direct and immediate reactor trip, presents no hazard to the integrity of the Reactor Coolant System and turbine cycle, even under the very conservative assumptions. Pressure relieving devices incorporated in the two systems are more than adequate to limit the maximum pressures. The integrity of the core is maintained by the fixed high pressurizer pressure trip for the loss of load transient under conditions with a positive moderator coefficient. The high pressure trip is not required for core protection with a negative moderator coefficient. 


\subsection{WASTE GAS ACCIDENT}

A waste gas accident is defined as the sudden, uncontrolled release of xenon and krypton fission gases from the radioactive waste gas collection system in the Reactor Auxiliary Building. The rupture of piping or decay storage tanks could result in a waste gas accident.

Based on the experience with other operational closed cycle reactors, it is expected that there will be no large amounts of gaseous activity in the coolant. However, as a conservative approach, design of the Radioactive Waste Disposal System is based on the assumption that one percent of the fuel rods could have small cladding defects, which would allow fission products to leak into the reactor coolant. Such postulated leaks would result in a buildup of radioactive waste gas activities in the coolant and in the waste gas collection system.

The principal source of radioactive gases in the Radioactive Waste Disposal System is the bleeding of coolant from the Reactor Coolant System for the purpose of reducing coolant boron concentration. This bleeding, exponential with time, will approximate 20 cubic feet of liquid per day during the early days of the core cycle and 2,000 cubic feet per day on the last day of the cycle. In addition, large quantities of coolant will be bled during startup after hot and cold shutdown.

Radioactive gases will be removed from the coolant bleed at the flash tank and at the gas stripper. The gases can then either be dispersed to the stack or compressed to $100 \mathrm{psig}$ and stored in the three waste gas decay tanks for dispersal to the stack at a later time.

Calculation of the maximum curies of waste gases which can exist outside the core at any time, based on a reference condition of one percent defective fuel rod cladding, is shown in Table 10.2. (Reference for the basis of this calculation is Paragraph 13.1 of this report, Fission Product Release From Fue1.)

Table 10. 2 Maximum Waste Gases

\begin{tabular}{|l|r|r|}
\hline $\begin{array}{l}\text { WASTE GAS } \\
\text { ISOTOPES }\end{array}$ & HALF-LIFE & CURIES \\
\hline Krypton 85m & 4.36 hours & 265 \\
Krypton 85 & 10.57 years & 2,556 \\
Krypton 87 & 78.00 minutes & 149 \\
Krypton 88 & 2.77 hours & 428 \\
Xenon 133 & 5.27 days & 32,200 \\
Xenon 135 & 9.20 hours & 897 \\
Xenon 138 & 17.00 minutes & 65 \\
\hline
\end{tabular}




\subsection{WASTE GAS ACCIDENT (Continued)}

The above values, except that for krypton 85, represent equilibrium between leakage and decay. Krypton 85 does not reach equilibrium because of its long half-life. The curies calculated for krypton 85 are the maximum accumulated curies during a core cycle and assume that defects are all in fuel rods undergoing their third core cycle of 345 days at full power.

Some of the above activity would remain in the coolant even after several system volumes of coolant bleed and some would have been dispersed earlier through the stack. However, if all of the waste gases processed by the Radioactive Waste Disposal System were accumulated and held in the waste gas decay tanks, the total radioactivity in all these tanks could be as much as 30,000 curies.

In the unlikely event of the rupture of a waste gas decay tank and its effect on the associated piping, under these postulated conditions, the contents of two tanks (approximately 20,000 curies) could be released to the concrete cubicle containing the waste gas decay tanks and to the enclosed lower operating area of the Reactor Auxiliary Building. Because released waste gas is discharged to the stack by normal operation of the ventilation system (one air change every six minutes), and because the Reactor Auxiliary Building is maintained at a slight negative pressure, no significant quantity of radioactive waste gases would esscape to the environment at ground level.

The consequences of such an uncontrolled release are evaluated in Paragraph 15.2, Other Malfunctions. Study results show that there would be no undue hazard to the pub1ic or plant personnel from this accident. 


\section{SECTION 11 \\ PLANT BEHAVIOR}

\subsection{LOSS OF POWER}

\section{1 .1 GENERAL}

As described in Paragraph 4.2, simultaneous loss of power from both utilities is not considered credible. The following discussion considers the loss of power and component failures in the auxiliary electric power system.

\subsubsection{MALFUNCTION IN THE 220-KV OR 138-KV SWITCHYARD}

Malfunction of any major item of equipment in the $220-\mathrm{kv}$ or $138-\mathrm{kv}$ switchyard would be detected by the appropriate bus differential relaying system or breaker backup relay system. In either event, the faulty component would be electrically isolated.

The 220-kv switchyard is of the double breaker-double bus design and operates with all circuit breakers closed. A fault or failure in the $220-\mathrm{kv}$ switchyard, or tripping of the generator 220-kv circuit breakers will not result in a loss of auxiliary electric system power supply. Power would continue to be supplied without interruption to the auxiliary electric power system through the $138-\mathrm{kv}$ switchyard and auxiliary transformer $\mathrm{C}$.

The most significant malfunction in the $138-k v$ switchyard would be an internal fault in either of the two $138-\mathrm{kv}$ circuit breakers feeding auxiliary transformer $C$. In the event of such a fault, both circuit breakers would trip and the power supply through transformer $\mathrm{C}$ would be lost. Power to station $4160-\mathrm{v}$ buses $\mathrm{IC}$ and $2 \mathrm{C}$ would then be momentarily interrupted and restored as described below under Auxiliary Transformer C Malfunction. The station is designed to accept loss of power from transformer $C$.

Thus any failure in the $220-k v$ or $138-k v$ switchyards will not result in sustained loss of auxiliary electric, power to the station.

\subsubsection{MALFUNCTION OF PLANT EQUIPMENT}

Malfunction of any major item of plant equipment, particularly electrical apparatus, will affect plant operation.

Possible malfunctions that could disrupt the plant auxiliary electric power system are as follows (refer to Figure 11.1):

a. Turbine or reactor malfunction

b. Generator, generator stepup transformer, auxiliary transformers $A$ and $B$, and the associated control or relaying equipment malfunctions (generator zone malfunctions) 



\title{
11.1.3 MALFUNCTION OF PLANT EQUIPMENT (Continued)
}

\author{
c. Auxiliary transformer $C$ or associated control or relaying equip- \\ ment malfunctions \\ d. Bus bar fault on 4160-v buses $1 \mathrm{~A}$ or $1 \mathrm{~B}$ \\ e. Bus bar fault on $4160-v$ buses $1 \mathrm{C}$ or $2 \mathrm{C}$ \\ f. $480-v$ system malfunction
}

\section{Turbine or Reactor Malfunction}

A malfunction in either the reactor or turbine cycle will trip both the reactor and turbine. After a trip, generator excitation is maintained to permit the turbine generator to supply coast-down energy to the reactor coolant pumps. The pumps and exciters will operate for about five minutes with the speed being reduced to 40 to 50 percent before their respective circuit breakers are tripped.

Following coast-down, power is restored to buses $1 \mathrm{~A}$ and $1 \mathrm{~B}$ from the 138-kv switchyard by closing tie-breakers $11 \mathrm{CO} 1$ and $12 \mathrm{CO} 1$ of the $4160-\mathrm{v}$ system In addition, the generator disconnect switch may be opened to allow the $220-\mathrm{kv}$ system to serve as a standby supply of power for the plant through the generator transformer and auxiliary transformer $A$ and $B$.

Loss of generation from a reactor or turbine malfunction does not cause a loss of power in the 220-kv and 138-kv switchyards. The 220-kv and 138-kv utility systems are always operated with sufficient spinning reserve in the inter-connected utility systems to accommodate the transient which would result from an unexpected loss of power from the station. The two utility systems ensure that power is available to the Station in the event of either reactor or turbine-generator malfunctions.

\section{Generator Zone}

Malfunctions within the generator zone are detected by one or more relay systems; differential, transformer sudden pressure, overcurrent, stator ground, or loss of field relay systems.

Operation of any one of the relay systems would immediately trip the generator $220-\mathrm{kv}$ and the auxiliary transformer $A$ and $B \quad 4160-v$ circuit breakers supplying buses $1 \mathrm{~A}$ and $1 \mathrm{~B}$. Thus, immediately after such a malfunction, the turbine and reactor are tripped and the generator zone is electrically isolated. Flywheel inertia of the reactor coolant pumps provides sufficient flow to prevent core damage.

Power can be restored to buses $1 \mathrm{~A}$ and $1 \mathrm{~B}$ from auxiliary transformer $\mathrm{C}$ by closing the $4160-\mathrm{v}$ bus-tie circuit breakers $11 \mathrm{C0} 1$ and $12 \mathrm{C01}$. At no time are buses $1 \mathrm{C}$ or $2 \mathrm{C}$ without power. 


\title{
11.1.3 MALFUNCTION OF PLANT EQUIPMENT (Continued)
}

\author{
Auxiliary Transformer C Trip
}

A malfunction associated with auxiliary transformer $C$ would be detected by one or more relay systems; differential, high-side overcurrent, and transformer sudden pressure relay systems.

The operation of any of these relay systems would immediately trip both the transformer $138-\mathrm{kv}$ and $4160-\mathrm{v}$ circuit breakers resulting in loss of power to buses $1 \mathrm{C}$ and $2 \mathrm{C}$.

Time delayed under-voltage relays on buses $1 \mathrm{C}$ and $2 \mathrm{C}$ are arranged to initiate the following automatic sequence in the event that the voltage on both buses drops below 70 percent for more than 20 seconds:

a. The turbine is tripped.

b. The generator is tripped, opening the 220-kv, 4160-v, and generator field breakers.

c. A11 4160-v motor circuit breakers are tripped to prepare the system for re-energizing.

d. The generator disconnect switch starts to open 30 seconds after the generator voltage has decayed to 40 percent. Operation is. complete in another 30 seconds.

e. Generator 4160-v circuit breakers $11 \mathrm{~A} 04$ and $11 \mathrm{~B} 04$ are closed.

f. 4160-v bus-tie breakers $11 \mathrm{C} 01$ and $12 \mathrm{CO} 1$ are closed and indicating lights inform the operator that the automatic sequence is complete.

At this point, auxiliary transformer $C$ and the generator are both electrically isolated, all $4160-\mathrm{v}$ motor circuits are tripped, and al1 4160-v and 480-v system transformers and tie circuit breakers are aligned to feed the auxiliary electric power system from the 220-kv system via the generator step-up transformer and auxiliary transformers $A$ and $B$. The total elapsed time from the initial malfunction is less than two minutes. The only action required to restore supply to the entire auxiliary electric power system is the closing of one or both of the $220-\mathrm{kv}$ generator circuit breakers from the control room.

A postulated second-order failure of disconnect switch d-c drive motor would necessitate manul operation, and could increase this time from two to approximately seven minutes.

\section{Bus Fault on $4160-\mathrm{V}$ Buses $1 \mathrm{~A}$ or $1 \mathrm{~B}$}

During on-line operation, a bus fault on bus $1 \mathrm{~A}$ or $1 \mathrm{~B}$ would be cleared by the overcurrent protection on circuit breakers $11 \mathrm{~A} 04$ or $11 \mathrm{~B} 04$ and the bus de-energized. 


\subsubsection{MALFUNCTION OF PLAN'T EQUIPMENT (Continued)}

During plant startup, buses $1 \mathrm{~A}$ and $1 \mathrm{~B}$ are fed, respectively, from adjacent buses $1 \mathrm{C}$ and $2 \mathrm{C}$ and the fault would be cleared, respectively, by circuit breakers $11 \mathrm{C} 01$ or $12 \mathrm{C} 01$.

Loss of power to bus $1 \mathrm{~A}$ results in the loss of reactor coolant pumps $A$ and $C$. Loss of power to bus $1 \mathrm{~B}$ results in the loss of reactor coolant pump $B$ and the generator exciter. Either or both of these incidents will result in turbine and reactor tripping action except at reactor power levels lass than 20 percent.

A fault on bus $1 \mathrm{~A}$ or $1 \mathrm{~B}$ would not affect buses $1 \mathrm{C}$ or $2 \mathrm{C}$ except that during startup, bus $1 \mathrm{C}$ or $2 \mathrm{C}$ feeding bus $1 \mathrm{~A}$ or $1 \mathrm{~B}$ would experience a momentary voltage dip which would last until the relays detect the fault and open tie breakers $11 \mathrm{C} 01$ and $12 \mathrm{CO}$.

\section{Bus Fault in $4160-\mathrm{V}$ Buses $1 \mathrm{C}$ or $2 \mathrm{C}$}

A bus fault on $4160-v$ buses $1 \mathrm{C}$ or $2 \mathrm{C}$ would be cleared by the overcurrent protection on circuit breakers $11 \mathrm{C} 02$ or $12 \mathrm{C} 02$ and the bus de-energized. Each of these buses feeds one complete safety injection train. Thus, loss of either bus still leaves the station with one complete safety injection train. Adequate physical separation ensures that a fault on one bus could not affect the other.

Loss of either bus $1 \mathrm{C}$ or bus $2 \mathrm{C}$ would result in a "loss of feedwater" trip because of loss of one feedwater train.

\section{$480-\mathrm{V}$ System Malfunction}

From a safety analysis standpoint, loss of power to the $480-\mathrm{v}$ system is of immediate concern only in that it supplies the motor operated valves associated with the safety injection system. The valves for one safety injection train are fed from $480-v$ bus 1 and the other from bus 2 . The three loop injection valves are common to both trains and are arranged to be supplied from either of the two buses by automatic transfer switches. The 480-v buses 1 and 2 receive power from 4160-v buses $1 \mathrm{C}$ and 2C. Relaying on all circuits provides for rapid isolation of malfunctioning components and the backup power sources ensure that no single malfunction in the $480-v$ system can render both sets of safety-injection motor operated valves inoperable.

\section{Summary}

It has been shown that loss of any single piece of major equipment or item of electrical apparatus will not interrupt the auxiliary power system for more than two minutes. If the major failure is compounded, with a second order failure of the generator disconnect switch $d-c$ drive, the maximum interruption is seven minutes. This interruption of power does not jeopardize safe plant operation, since the plant can accept loss of power for a period in excess of 15 minutes without loss of decay heat removal capacity. 


\subsection{EARTHQUAKES}

\subsubsection{GENERAI}

The purpose of this section is to show that the behavior of the plant when subjected to an earthquake will not impair the safe operation or an orderly shutdown of the plant.

\subsubsection{ASEISMIC DESIGN CRITERIA}

A11 components, systems, and structures which are important to the nuclear safety of the plant were designed in accordance with the following criteria:

a. The design earthquake for the site was determined from the results of a seismic investigation made by Dames and Moore, Consultants in Applied Earth Sciences. Based on earthquake history and the site location, a maximum ground acceleration of $0.25 \mathrm{~g}$ was established.

b. Primary steady state stresses when combined with seismic stresses shal1 be maintained within the allowable working stress range based upon the response to a ground motion having a maximum acceleration of $0.25 \mathrm{~g}$.

c. Combined stresses, including seismic stresses based upon the response to a ground acceleration of twice the above value $(0.5 \mathrm{~g})$, are such that the function of the component, system, or structure is not impaired, and a safe and orderly shutdown of the plant is assured.

d. The analysis of the dynamic loads imparted by the maximum ground acceleration resulting from an earthquake were performed using the response spectrum approach. This analysis was applied for all components and structures considering their natural periods and using appropriate damping factors. Figure 11.2 shows a loglog plot of the acceleration response spectra normalized to a maximum ground acceleration of $0.25 \mathrm{~g}$ for various percentages of critical damping. The input data for these curves were developed by Dr. G. W. Housner. These curves cover periods from 0.01 to 1.0 seconds and damping factors of one-half percent to seven percent. The horizontal component of the ground acceleration was taken directly from the curves of Figure 11.2 and the vertical component was taken as two-thirds of this value.

e. A tabulation of damping factors which were used for various vibratory systems important to the nuclear safety of the plant is presented in Table 11.1. Conservative values are shown for systems of various materials, methods of construction, and location with respect to the ground. These are typical of the damping factors utilized for the plant design. 


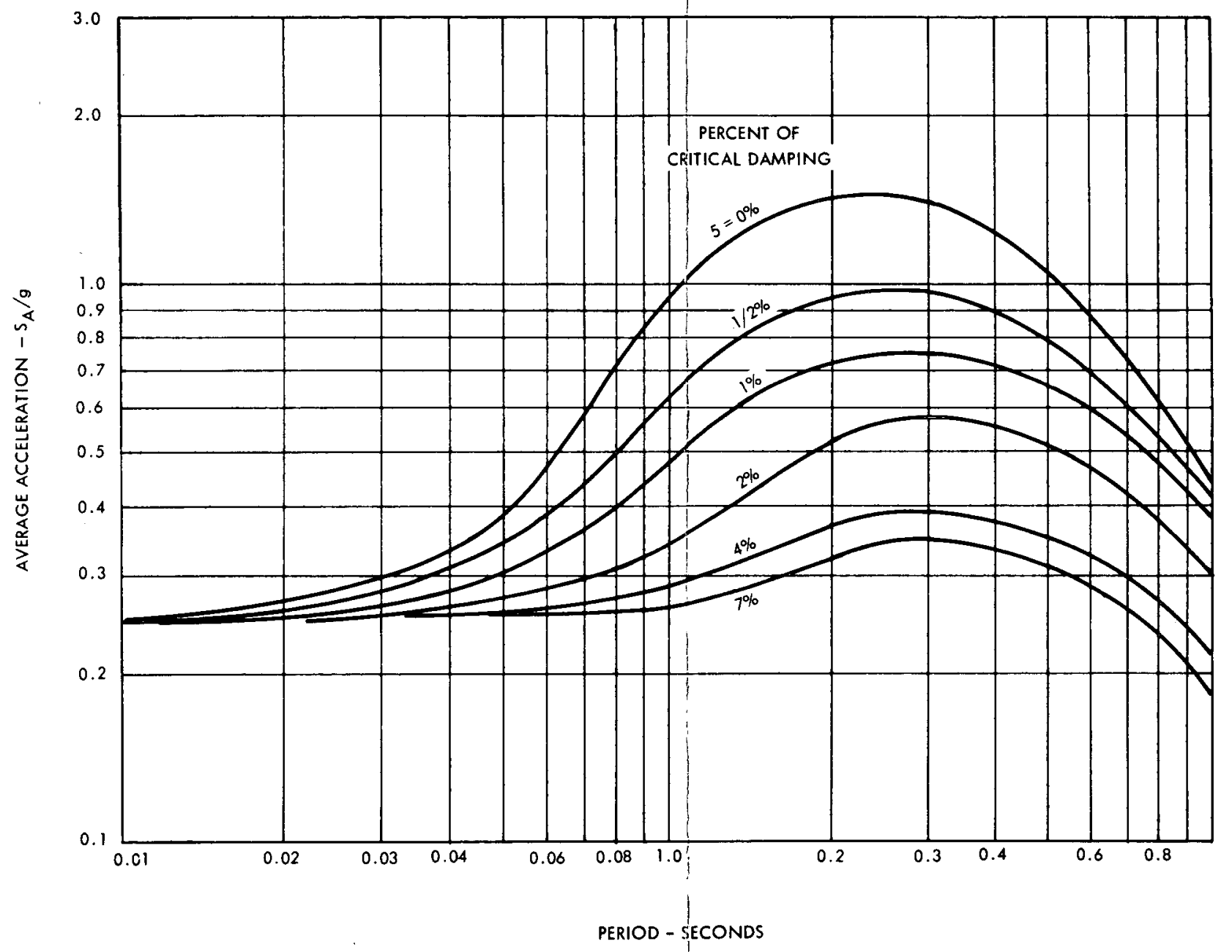

FIGURE 11.2 AVERAGE ACCELERATION RESPONSE SPECTRA NORMALIZED FOR $0.25 \mathrm{~g}$ GROUND ACCELERATION 
Table 11.1 Damping Factors

\begin{tabular}{|c|c|}
\hline COMPONENT OR STRUCTURE & $\begin{array}{c}\text { PERCENT OF } \\
\text { CRITICAL DAMPING }\end{array}$ \\
\hline $\begin{array}{c}\text { Reactor Vessels Internals } \\
\text { (Stainless Steel Core Support Structure) }\end{array}$ & 1.0 \\
a. Welded Assemblies & 2.0 \\
b. Bolted Assemblies & 4.0 \\
Reinforced Concrete Reactor Support \\
Structure Including the Reactor Vesse1 \\
Vital Piping Systems \\
Steel Containment Vesse1 and Foundation \\
Framed Steel Structures \\
Concrete Structures Above Ground \\
a. Shear Wall Type
\end{tabular}

\subsubsection{CLASSIFICATION OF COMPONENTS, SYSTEMS, AND STRUCTURES}

The objective of the seismic design of the plant is to verify that the most adverse conditions of stress and deflection resulting from the combined influence of normal and earthquake loads will not impair safe operation or orderly shutdown of the plant. Since the components, systems, and structures are not equally important for plant safety, these items are classified into the following three categories. Category A: components, systems, and structures that are important to the nuclear safety of the plant are designed in accordance with the above criteria. Category B: components, systems, and structures that are important to the continuity of power generation or whose contained activity is such that release would not constitute a hazard, are designed for a maximum ground acceleration of $0.2 \mathrm{~g}$. Category C: all remaining structures not directly associated with power generation are designed in accordance with the "Uniform Building Code." The above seismic design classifications were nessary to ensure a consistent approach in the design of the plant.

Tables 11.2 through 11.5 establish the categories applied to the various components, systems, and structures. Since the Category A items are the most important, the following parameters were used to place components, systems, and structures in this category: 
Table 11.2 Categorization of Reactor Cycle Equipment

\begin{tabular}{|c|c|c|}
\hline SYSTEM & CATEGORY & REMARKS \\
\hline \multicolumn{3}{|l|}{$\begin{array}{l}\text { Chemical and Volume } \\
\text { Control System }\end{array}$} \\
\hline Regenerative Heat Exchanger & $\mathrm{A}$ & $\begin{array}{l}\text { Needed for orderly shutdown, } \\
\text { boric acid injection }\end{array}$ \\
\hline Excess Letdown Heat Exchanger & $\mathrm{B}$ & \\
\hline Sea1 Water Heat Exchanger & A & $\begin{array}{l}\text { Needed for orderly shutdown, } \\
\text { maintenance of reactor coolant } \\
\text { pump seal flow }\end{array}$ \\
\hline Mixed Bed Demineralizers & $\mathrm{A}$ & $\begin{array}{l}\text { By connection to volume control } \\
\text { tank }\end{array}$ \\
\hline Volume Control Tank & $\mathrm{A}$ & $\begin{array}{l}\text { Needed for orderly shutdown, as } \\
\text { part of charging letdown and } \\
\text { seal water injection loop }\end{array}$ \\
\hline Chemica1 Mixing tank & $\mathrm{C}$ & \\
\hline Boric Acid Tank & $\mathrm{A}$ & Needed for boric acid injection \\
\hline Batching Tank & $B$ & \\
\hline Reactor Coolant Filter & A & $\begin{array}{l}\text { Needed for orderly shutdown, as } \\
\text { part of the letdown line }\end{array}$ \\
\hline Seal Water Filter & A & $\begin{array}{l}\text { Needed for orderly shutdown main- } \\
\text { tenance of reactor coolant pump } \\
\text { seal flow }\end{array}$ \\
\hline Charging Pumps & A & Needed for boric acid injection \\
\hline
\end{tabular}


Table 11.2 Categorization of Reactor Cycle Equipment (Continued)

\begin{tabular}{|c|c|c|}
\hline SYSTEM & CATEGORY & REMARKS \\
\hline Test Pump & A & $\begin{array}{l}\text { Backup pump for boric acid } \\
\text { injection }\end{array}$ \\
\hline Boric Acid Injection Pumps & A & Needed for boric acid injection \\
\hline Boric Acid Transfer Pumps & A & Needed for boric acid injection \\
\hline Boric Acid Filter & A & Needed for boric acid injection \\
\hline \multicolumn{3}{|l|}{ Sampling System } \\
\hline Sample Heat Exchanger & B & \\
\hline Delay Coil & $\mathrm{B}$ & \\
\hline Reactor Coolant Sample Vesse1 & B & \\
\hline $\begin{array}{l}\text { Volume Control Tank } \\
\text { Sample Vessel }\end{array}$ & B & \\
\hline \multicolumn{3}{|l|}{ Auxiliary Coolant System } \\
\hline Residual Heat Exchanger & A & $\begin{array}{l}\text { Needed for orderly shutdown as } \\
\text { part of letdown line }\end{array}$ \\
\hline $\begin{array}{l}\text { Component Cooling Heat } \\
\text { Exchangers }\end{array}$ & A & $\begin{array}{l}\text { Needed for orderly shutdown and } \\
\text { safety injection }\end{array}$ \\
\hline Spent Fuel Pit Heat Exchanger & B & \\
\hline Component Cooling Surge Tank & A & $\begin{array}{l}\text { Needed for orderly shutdown to } \\
\text { maintain NPSH on component } \\
\text { cooling pumps }\end{array}$ \\
\hline Component Cooling Pumps & A & Needed for safety injection \\
\hline Spent Fuel Pit Pump & B & \\
\hline Residual Heat Removal Pump & A & $\begin{array}{l}\text { Needed for orderly shutdown, as } \\
\text { part of letdown pump system }\end{array}$ \\
\hline
\end{tabular}


Table 11.2 Categorization of Reactor Cycle Equipment (Continued)

\begin{tabular}{|c|c|c|}
\hline SYSTEM & CATEGORY & REMARKS \\
\hline \multicolumn{3}{|l|}{ Safety Injection System } \\
\hline Safety Injection Pumps & $\mathrm{A}$ & Needed for safety injection \\
\hline Recirculation Pumps & A & Needed for safety injection \\
\hline Recirculation Heat Exchanger & $\mathrm{A}$ & Needed for safety injection \\
\hline \multicolumn{3}{|l|}{ Reactor Coolant System } \\
\hline Reactor Vessel and Internals & A & Nuclear safety of the plant \\
\hline Steam Generators & A & Nuclear safety of the plant \\
\hline Reactor Coolant Pumps & A & Nuclear safety of the plant \\
\hline Pressurizer & A & Nuclear safety of the plant \\
\hline Control Rod Drive Mechanism & A & Nuclear safety of the plant \\
\hline Safety and Relief Valves & A & Nuclear safety of the plant \\
\hline Pressurizer Relief Tank & A & Nuclear safety of the plant \\
\hline
\end{tabular}

Table 11.3 Categorization of Structural Equipment

\begin{tabular}{|c|c|c|}
\hline STRUCTURES & CATEGORY & REMARKS \\
\hline Reactor Structure and Containment & A & $\begin{array}{l}\text { Needed for nuclear safety and } \\
\text { order ly shutdown }\end{array}$ \\
\hline Spent Fuel Pit & A & Needed for nuclear safety \\
\hline Reactor Auxiliary Building & A & $\begin{array}{l}\text { Needed for nuclear safety and } \\
\text { orderly shutdown }\end{array}$ \\
\hline \multicolumn{3}{|l|}{ Main Building } \\
\hline Control Room & A & $\begin{array}{l}\text { Control of orderly shutdown and } \\
\text { safety injection }\end{array}$ \\
\hline Switchgear Room & A & $\begin{array}{l}\text { Auxiliary electric power for } \\
\text { orderly shutdown and safety } \\
\text { injection }\end{array}$ \\
\hline
\end{tabular}


Table 11.3 Categorization of Structural Equipment (Continued)

\begin{tabular}{|c|c|c|}
\hline STRUCTURES & CATEGORY & REMARKS \\
\hline Battery Room & A & $\begin{array}{l}\text { D-C control power for orderly } \\
\text { shutdown and safety injection }\end{array}$ \\
\hline Turbine Support Pedestal & A & Connection to main steam lines \\
\hline Heater Platforms & B & \\
\hline Intake Structure & A & $\begin{array}{l}\text { To the extent that water is } \\
\text { always available to the salt } \\
\text { water cooling pumps. Otherwise } \\
\text { Category B }\end{array}$ \\
\hline
\end{tabular}

Table 11.4 Categorization of Miscellaneous Mechanical Equipment

\begin{tabular}{|l|c|c|}
\hline \multicolumn{1}{|c|}{ MECHANICAL EQUIPMENT } & CATEGORY & REMARKS \\
\hline Refueling Water Storage Tank & A & Required for safety injection \\
Primary Plant Makeup Tank & B & \\
Feedwater Pumps & A & Required for safety injection \\
Salt Water Cooling Pumps & A & $\begin{array}{c}\text { Required for residual heat } \\
\text { removal }\end{array}$ \\
$\begin{array}{l}\text { Primary Drain Tank Pump } \\
\text { Sphere Cooling Units }\end{array}$ & B & Available for heat removal from \\
Sphere Sump Pump \\
$\begin{array}{l}\text { Equipment in the Radioactive } \\
\text { Waste System }\end{array}$
\end{tabular}


Table 11.4 Categorization of Miscellaneous Mechanical Equipment (Continued)

\begin{tabular}{|l|c|c|}
\hline \multicolumn{1}{|c|}{ MECHANICAL EQUIPMENT } & CATEGORY & REMARKS \\
\hline $\begin{array}{l}\text { Decontamination Drain Tank } \\
\text { and Pump }\end{array}$ & B & \\
$\begin{array}{l}\text { Radioactive Waste } \\
\text { Ion-Exchangers }\end{array}$ & B \\
Spent Resin Storage Tank & B \\
Gas Stripper and Pump & B \\
Monitor Tanks and Pumps & B \\
Waste Gas Surge Tank & B & \\
Waste Gas Compressor & B & A \\
Waste Gas Decay Tanks & Prevent release of high level gase- \\
ous activity to the environment
\end{tabular}

Table 11.5 Categorization of Electrical Equipment

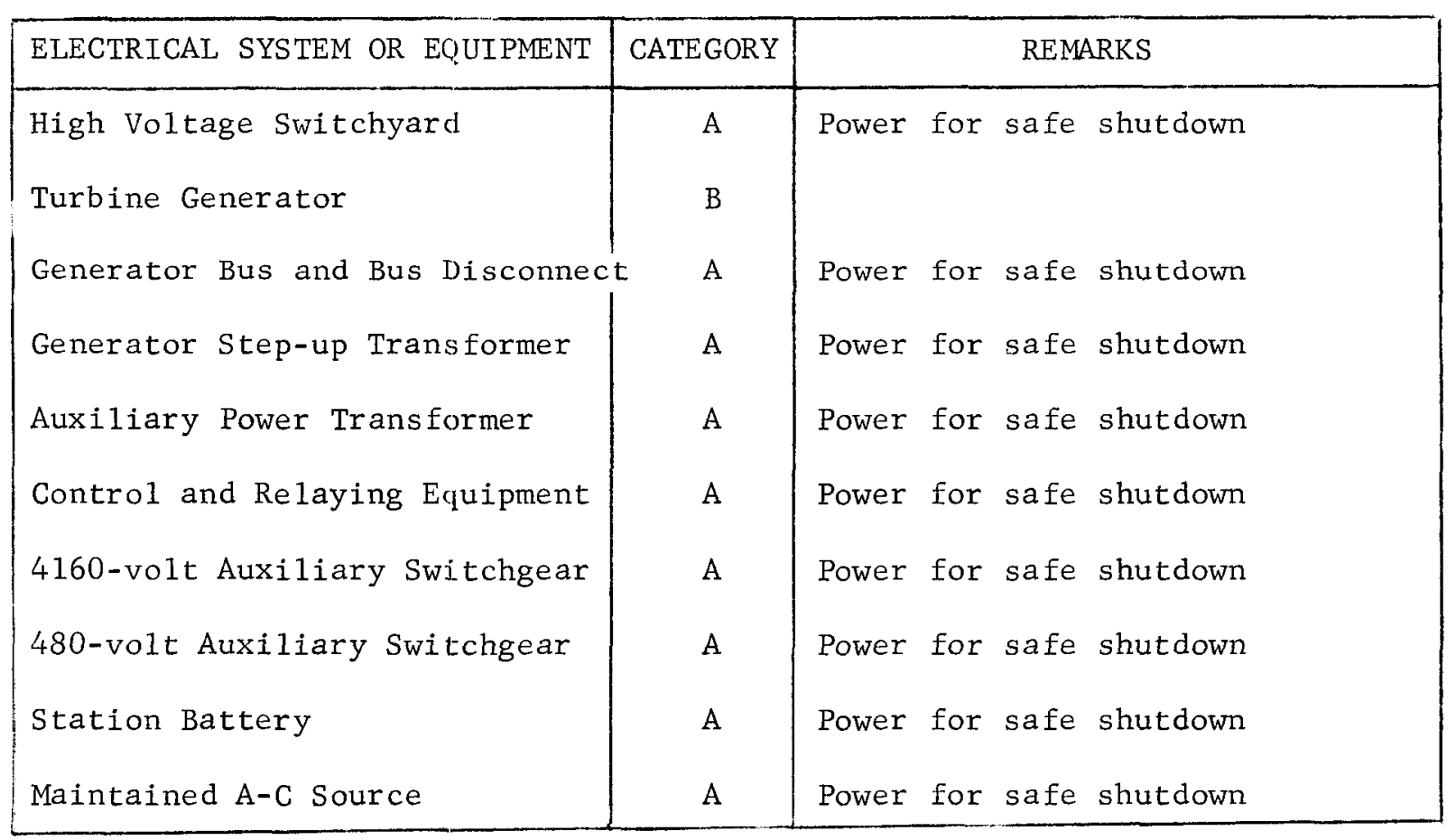




\subsubsection{CLASSIFICATION OF COMPONENTS, SYSTEMS, AND STRUCTURES (Continued)}

a. Those items whose failure would result in an unrestricted release of radioactive products.

b. Those items needed for safety injection or recovery from a loss of coolant accident.

c. Those items needed to insure the safe and orderly shutdown on the unit.

\subsubsection{METHOD OF ANALYSIS}

The following method of analysis has been applied to components, systems, and structures in category $\mathrm{A}$ :

a. The natural periods of vibration of the structure, component, or system were determined.

b. Average acceleration response values for the specific periods were determined from Figure 11.2, using appropriate damping factors.

c. Stresses and deflections resulting from the combined influence of normal loads and the additional earthquake loads were calculated to determine that continued safe operation of the plant is not impaired.

d. The maximum response value (peak of the curve) corresponding to the appropriate damping factor was used in performing the stress analysis for vibratory systems of a highly complex nature. By using this conservative value and demonstrating that the stresses are satisfactory, it became unnecessary to perform any further analysis to determine the natural periods of vibration for the system.

e. Piping

1. An acceleration corresponding to the peak of the $0.25 \mathrm{~g}$ normalized response curve was used for hot piping and all piping penetrating the vapor containment sphere subject to expansion movements. The stresses at the peak for carbon steel based on $1.0 \mathrm{~g}$ horizontal and $0.67 \mathrm{~g}$ vertical components acting simultaneously were combined with the stresses due to weight and internal pressure at cricical points. The maximum combined stress at any point was calculated to determine that the stress is maintained below the Code allowable stress.

2. The natural frequency of the segmental lengths for piping that could be held fixed and still meet the Code allowable expansion stress range by stiffening with the addition of anchors and guides was calculated individually and the seismic response read from the $0.25 \mathrm{~g}$ normalized response curve. The maximum combined stress was computed and compared with the Code allowable stress. 


\subsubsection{EXAMPLES OF ASEISMIC DESIGN FOR CRITICAL COMPONENTS}

\section{Reactor Vesse1 and Steam Generator Supports}

\section{Reactor Vesse1 Supports}

The reactor vesse1 is supported by three brackets that are integral with the vesse1. These brackets are set in support shoes that allow radia1 movement caused by thermal expansion but prevent horizontal rotation or translation of the vesse1. Each support shoe is attached to a vertical column and a horizontal bracket that respectively transmits the vertical and horizontal loads from the vessel to the reactor building foundation.

\section{Steam Generator Supports}

Each steam generator is supported by two separate sets of supports. One set transmits the vertical loads from the vessel to the foundation and the other set transmits the horizontal loads from the vessel to the foundation.

The supports are frames consisting of structural steel beams and columns partially embedded in the walls of the reactor building. Vertically, the steam generator is hung from the frame by hanger rods seated in spherical bearing washers. This arrangement permits movement caused by thermal growth of the piping system. Horizontally, the vessel is supported by a system of hard stops in the direction of no movement and hydraulic snubbers in the direction of thermal movement. The snubbers will permit the slow movements associated with thermal growth but provide hard stops if subjected to acceleration from earthquake forces.

The supports are designed to limit the seismic response amplification factor to 1.0 , which results in deflections being maintained within an acceptable range for both the $0.25 \mathrm{~g}$ and $0.5 \mathrm{~g}$ earthquakes. The stress levels obtained by combining the stresses resulting from gravity loads, piping reactions, operating loads, and seismic forces from the $0.25 \mathrm{~g}$ earthquake components are less than the allowable working stresses prescribed by the American Institute of Steel Construction and the American Concrete Institute. For the above loading when combined with the seismic loading from the $0.5 \mathrm{~g}$ earthquake components, the resulting stress levels of the steel structures are within the elastic range of the material. In analyzing the seismic load, the reactor coolant pipe was not considered to carry any part of the load.

\section{Containment Vesse1}

The natural period of the sphere was determined; then, using the appropriate damping factor (4 percent critical damping), an amplification factor of 1.0 was determined from the acceleration response spectra curves. From this data, an equivalent horizontal static force equal to 25 percent of the structure's dead weight and an equivalent vertical static force equal to two-thirds of the horizontal force were applied to the sphere. The resulting stresses were combined with those from other causes to determine the maximum stresses. 


\subsubsection{EXAMPLES OF ASEISMIC DESIGN FOR CRITICAL COMPONENTS (Continued)}

The sphere was designed as a pressure vessel to resist the stresses induced by pressure and temperature in combination with the earthquake forces and gravity loads.

The following table summarizes the primary stresses at grade, since these are representative of the peak stress values of the sphere.

Table 11.6 Summary of Plate Stresses at Grade

EARTHQUAKE: HORIZONTAL ACCELERATION $=0.25 \mathrm{~g}$; VERTICAL ACCELERATION $=0.167 \mathrm{~g}$

\begin{tabular}{|c|c|c|}
\hline SOURCE OF STRESS: & TENSION (psi) & COMPRESSION (psi) \\
\hline $\begin{array}{l}\text { Earthquake horizontal acceleration } \\
\text { Earthquake vertical acceleration } \\
\text { Internal pressure of } 46.4 \text { psig } \\
\text { Internal vacuum of } 2.0 \text { psig }\end{array}$ & $\begin{array}{r}163 \\
87 \\
18,800 \\
-0- \\
\end{array}$ & $\begin{array}{r}163 \\
87 \\
-0- \\
810 \\
\end{array}$ \\
\hline Totals & 19,050 & 1,040 \\
\hline $\begin{array}{l}\text { Allowable ASME stresses for above } \\
\text { conditions }\end{array}$ & 19,250 & 2,292 \\
\hline $\begin{array}{l}\text { In accordance with the ASME Code } \\
\text { stresses due to the weight of the } \\
\text { she } 11 \text { are not included in the } \\
\text { above summary. }\end{array}$ & & \\
\hline These stresses are: & 515 & 413 \\
\hline
\end{tabular}

Feedwater Piping

The feedwater piping from its penetration of the sphere to its connection to Steam Generator No. E-1C, Line No. 326-10"-EG, is supported on two variable springs, two rod hangers, and one guide. Vertical and horizontal components of the seismic acceleration, taken as $0.67 \mathrm{~g}$ and $1.0 \mathrm{~g}$, respectively, are absorbed by the guide, two rod hangers, and four hydraulic shock suppressors. The stress range due to thermal expansion with all restraints considered is 4,210 psi when computed according to the rules of the ASA Code for Pressure Piping ASA B31.1.

The longitudinal stress due to internal pressure is 4,920 psi. The maximum stress due to weight and seismic effects occurs at the third elbow upstream from the steam generators; its value is 9,040 psi. The combined sustained stress is therefore $13,960 \mathrm{psi}$ at the elbow in question. In the computerized solution of the loading effects, the flexibility and stress intensification factors prescribed by ASA B31.1 are employed. 


\subsubsection{EXAMPLES OF ASEISMIC DESIGN FOR CRITICAL COMPONENTS (Continued)}

Under the ASA B31.1 Section 1 rules, the thermal stress range may not exceed 22,500 psi, and the sustained stresses may not exceed 15,000 for the material from which the feedwater piping is fabricated. Neither of these values was exceeded at any point in the system.

Refueling Water Tank

A hydrodynamic analysis of the refueling water tank was made using the method outlined in Chapter 6, "Nuclear Reactors and Earthquakes" (Atomic Energy Commission publication). Shears and bending moments were obtained from this analysis for both the $0.25 \mathrm{~g}$ and $0.5 \mathrm{~g}$ earthquakes. The forces from the $0.25 \mathrm{~g}$ seismic load were combined with the primary steady state stresses and the resulting stress levels were below those prescribed by the American Petroleum Institute - "Welded Steel Tanks for Oil Storage." For the $0.5 \mathrm{~g}$ earthquake forces combined with the primary steady state stresses, the resulting stresses were within the elastic range for the materials or below other stress limiting conditions such as buckling.

The weight of the stored water is used in maintaining stability against overturning of the tank during an earthquake. To take advantage of this weight, the tank has been anchored to a reinforced concrete foundation that is designed to distribute the weight of the water to the tank perimeter to counteract the uplift.

\section{$\underline{\text { Reactor Coolant Pump Supports }}$}

The reactor coolant pumps are supported on variable load spring hangers which have been modified so that they behave as a solid rod under maximum operating temperature conditions.

In the discussions of reactor coolant pump supports, reactor cooling piping, pressurizer, and reactor internals and core, horizontal and vertical accelerators were assumed to act simultaneously. Seismic loadings were evaluated as steady state loadings. The seismic stress, when combined with other loadings, remained within the limits established by the ASME Boiler and Pressure Vesse1 Code and the ASA Code for Pressure Piping.

The natural frequency of the support rods was calculated and the response to the design earthquake with a one-half percent damping factor was determined. No transfer of vertical load to the piping was considered. All of the horizonta'l seismic load of the pump mass is taken by the piping.

Stress limits in the supports were limited to those permitted for supports in the ASA Code for Pressure Piping.

\section{Reactor Coolant Piping}

The reactor coolant piping was assumed to respond at the peak of the curve with one-half percent damping for the design earthquake. In addition, the vertical deflection of the steam generator supports and the reactor coolant pump supports, together with the horizontal seismic loading of the pump (peak of the curve response) were included in a piping flexibility program to evaluate the reactions and the end motion to the design seismic loading. 


\subsubsection{EXAMPLES OF ASEISMIC DESIGN FOR CRITICAL COMPONENTS (Continuied)}

In all cases stresses were maintained within the allowable stress limits as set forth in the ASA Code for Pressure Piping.

\section{Reactor Vesse1}

Design Criteria

The reactor vessel is designed to withstand accelerations of $0.33 \mathrm{~g}$ occurring in both the horizontal and vertical directions. Using criteria developed for the site subsequent to the design of the reactor vessel, the seismic design criteria for the site was developed. Simplification of the ground acceleration was further considered according to an interpolated response spectrum. The vessel has a natural period of vibration of $0.024 \mathrm{sec}-$ ond which would correspond to a horizontal response acceleration, with credit for damping, of $0.28 \mathrm{~g}$ according to the design spectra. Thus, the assumed loading of $0.33 \mathrm{~g}$, acting simultaneously in both the vertical and horizontal directions, is conservative. The combined stresses, including seismic, based upon the response to a ground acceleration of twice the design value, were also evaluated to ensure that vessel integrity would not be impaired.

\section{Vessel Movement}

In the case where the horizontal component of the earthquake is acting on the vesse1, two effects were encountered. The vessel is restricted from moving sideways by the radial stops and support keys which are part of the support structure. A horizontal force is therefore imposed at two of the support locations simultaneously, due to the vessel inertia.

\section{Nozzles}

An earthquake will also cause reactions at the reactor vessel nozzles due to movement of piping connected to the nozzles. Because the piping and the pump in the Reactor Coolant System do not constitute a rigid structure, a dynamic analysis was performed. It was assumed that the principal mode of vibration of the piping is at the frequency giving the maximum response according to the response spectrum for a structure with 0.5 percent critical damping. The actual analysis of piping reaction on the reactor vessel nozzles, performed prior to setting the base ground acceleration inertia at $0.25 \mathrm{~g}$, assumed an amplified loading on the piping of $1.85 \mathrm{~g}$.

The total reactor vessel nozzle reactions were calculated with the aid of a piping flexibility computer program which has been used extensively and successfully in doing vibration analysis on piping system. These forces, together with the vessel inertia forces during an earthquake, form a couple which tries to overturn the vessel. The result is a vertical restoring couple at the vessel supports. In the case of a vertical earthquake load, the effect would be to increase or decrease the vertical load at each vessel support. Both horizontal and vertical components of the earthquake were assumed to occur simultaneously in the worst possible combination which would result in increased downward loads at the reactor vessel supports. 


\subsubsection{EXAMPLES OF ASEISMIC DESIGN FOR CRITICAL COMPONENTS (Continued)}

Ful1 details of the various loadings are given in the Reactor Vessel Design Report, July, 1965.

\section{Pressurizer}

The pressurizer is supported near its lower end by a skirt which is bolted to a steel structure integral with the concrete in the containment vessel. The pressurizer is a very rigid cantilevered vessel so that the response to ground motion is at the level of the design earthquake. The bolting is designed to withstand the shear loads and the tensile forces resulting from horizontal and vertical seismic motion without exceeding standards of steel construction for buildings.

\section{Reactor Internals and Core}

The internals and core were analyzed to determine the stresses and deflections caused by the design earthquake. The core support structure is supported at its upper flange from a ledge in the reactor vessel head flange and its lower end is restrained in its transverse movement by a radial support system attached to the vessel wall. Transverse loads are carried by the core barrel shell to be shared by the six lug and clevis joints. The thermal shield is fixed to the core barrel at its lower end and its upper end is keyed to the core barrel in much the same arrangement as the core barrel radial support system.

The seismic stresses were combined with the other primary steady state stresses and were within the allowable working stress range for the material and part under consideration. The design is such that the deflections, when combined with the deflections from other loadings, are sufficiently small, such that normal operation is not prevented. Considering a ground acceleration of twice the design earthquake, the stresses and deflections are such that the function of the various parts is not impaired and the reactor can be safely shut down. 


\subsection{FLOODS}

\subsubsection{FOOTHILL DRAINAGE}

As discussed at length in Paragraph 2.2, Meteorology, the plant site is not subject to large quantities of rainfall runoff. San Onofre Creek, approximately 1-1/2 miles north of the plant, drains most of the foothills. This natural drainage feature protects the plant site from the possibility of large floods. However, approximately 110 acres of the foothill area directly east of the plant will drain through the plant site to the ocean.

Drainage from the 110-acre watershed is computed to be $266 \mathrm{cfs}$, based on a storm that might occur once in 100 years. Runoff from the 110-acre watershed discharges through culverts under the railroad and Highway 101 into a 42-inch pipeline inlet structure.

The culverts will pass $230 \mathrm{cfs}$. Should a 100 year storm produce the calculated 266 cfs, the excess water will drain north from the culverts to drainage ditches along both the highway and the railroad tracks to a drainage outlet to the ocean located far north of the plant site.

The 42-inch concrete pressure pipe from the inlet structure conducts the water down the hill and underground through the north end of the site to the beach. The 42-inch pipe has a capacity of 270 cfs. This capacity is more than adequate to carry the maximum runoff that will reach the pipe.

\section{3 .2 SWITCHYARD DRAINAGE}

Surface drainage from the switchyards is collected in a paved, open ditch along the east side of the switchyards. This ditch discharges into the 42-inch pipe leading to the beach. Runoff from the switchyards is reduced and delayed because of the layer of San Mateo sand and crushed rock covering the area.

\subsubsection{ACCESS ROAD DRAINAGE}

Access roads to the site are bench-cut into the slopes surrounding the plant area. These roads are drained by paved side ditches that follow the roads. The ditches carry runoff to the ocean in a paved, open ditch along the north side of the plant. The side ditches are more than adequate to accommodate runoff from the roads.

\subsubsection{PLANT SITE DRAINAGE}

The yard area to be drained is approximately 6.5 acres. Runoff from this area is computed to be $13 \mathrm{cfs}$, based on a 100 year storm.

Yard areas are graded into low areas surrounding the buildings and structures. Each low area drains to a concrete catch basin with an open 


\subsubsection{PLANT SITE DRAINAGE (Continued)}

grating top. Several catch basins are connected by reinforced concrete pipe that discharges to the ocean.

The concrete pipe increases in size from 12 to 21 inches inside diameter as successive drainage areas are added. The system is adequate for plant area runoff without ponding at the catch basin. 


\subsection{LANDSLIDES}

The plant site has been excavated from the original seacliffs which lie along the coast. The inclination of slope cuts were designed and constructed for the site, consistent with good engineering practice. The possibility of 1 andslides was considered. The most direct evidence is that the cut slopes are consistent wi.th the natural vertical bluffs along the adjacent shoreline which appear to have safely withstood any destructive geologic processes. In order to utilize the relative shear strengths of the two distinct strata offered by the geologic profile of the cut slopes, the bench design approach was adopted.

The as-constructed slope profile consists of a 15-foot bench along the horizontal interface between the massive and very strong San Mateo sands, which comprise the lower 25 feet of the cut slope, and the terrace deposits above. The cuts were excavated to a slope of one-half to one, horizontal to vertical, above and below the bench.

Slope stability analyses indicate that when steady state stresses are combined with the seismic stresses which would result from a ground motion having a maximum acceleration of $0.25 \mathrm{~g}$, ravelling of thin wedges of the terrace material may slide onto the bench below. The San Mateo sands will not be affected. The highly compacted dense nature of the sands precludes the development of liquifaction as a failure mechanism during an earthquake.

Similar analyses indicate that the overall performance of the highest cut slopes subjected to an earthquake producing a maximum acceleration of twice the above value $(0.5 \mathrm{~g})$, is such that catastrophic, deep-seated slides will not occur. Slumping of terrace materials from the upper portions of the slope onto the bench might cause a small wedge failure of the lower san Mateo slopes because of overstressing due to the surcharge from above. The resulting thin slide mass would be so small that it would not affect the plant or switchyard structures.

Erosion of the cut slopes will not threaten the safe operation of the plant. Periodic inspection and preventative maintenance will preclude the development of significant erosion tendencies.

Seepage loss through the service water reservoir lining and through underlying terrace deposits would be minimal under the most conservative assumption. The four-foot thickness of the reservoir lining ensures that cracks would not extend through the entire blanket. Should leaks develop, the leaks would not constitute a threat to the safety of the slope adjacent to the reservoir. Seepage would percolate vertically and ultimately would be interrupted by and dissipated within the porous granular strata toward the ocean. 
11.5 FIRE

\section{5 .1 GENERAL}

The fire protection system consists of the equipment used to protect yard areas, structures, and the inside of buildings. Both wet and dry type equipment is provided. The equipment for each plant location was chosen after considering the area to be protected.

A water main and hydrant system is the primary source of protection in the yard. Water dispensing devices consist of hose reels inside the buildings for general protection and spray nozzles for hazard areas. Portable dry units supplement the water system.

\subsubsection{SOURCE OF WATER}

Two flash evaporators furnish the entire plant water supply. There is no connection to an outside water source or system. Water from the evaporators is stored in the three million gallon, asphalt-lined, earth reservoir on the bench approximately 75 feet above the plant grade.

Two fire pumps supply the plant fire protection system with water from the reservoir. One fire pump is capable of supplying the system design demand. The second pump is a standby and adds safety to the system. To reduce the possibility of power failure to both pumps, each fire pump is powered from a different bus of the 480-volt plant auxiliary power system.

Norma11y, the fire protection loop is pressurized by the service water system through a one inch tie line. The fire pumps are started automatically when a fire hydrant or hose is opened. The increased water demand causes a pressure drop in the header which closes a pressure switch and thus actuates one fire pump. A further pressure drop in the header will close another pressure switch in the fire protection loop and start the second pump. Fire pump operation is annunciated in the Control Room. Fire pumps are stopped manually from the Control Room.

An alternate, emergency supply of fire protection water is available from the two screen-wash pumps in the intake structure. These pumps supply sea water at 1,000 gallons per minute and a 275 foot head. One pump can be powered by an auxiliary gasoline engine if required.

\section{5 .3 YARD SYSTEM}

The yard system consists of the two fire pumps and underground cast-iron pipe supplying wet-barrel "California" type hydrants. The underground pipe serves the main plant structures in a loop around the plant. Other buildings and areas are served with single pipe runs from the loop. The two fire pumps, rated at 1,000 gallons per minute and a 275 foot head, take suction from the reservoir. The yard system is designed to supply four, two and one-half inch hydrant outlets at one time from any given single eight inch take-off line at a minimum flowing pressure of 50 psig. This 


\subsubsection{YARD SYSTEM (Continued)}

design pressure is supplied at elevation 110 feet in the switchyards and is exceeded in the plant areas at elevation 20 feet.

The pipe loop around the plant is cross-connected by a pipe through the plant to form two loop sections. Sectionalizing valves make it possible to isolate one section of the system for maintenance while service is maintained inside the plant and in other yard areas. The piping loop supplies water to each given point through more than one pipe. This makes a greater quantity of water available at reduced head loss and gives added reliability and safety to the system.

\subsubsection{CONTROL-ADMINISTRATION BUILDING}

The interior of the Control-Administration Building is protected by both wet and dry fire equipment. Fire hose cabinets, each containing 50 feet of lined, cotton hose and a brass adjustable stream or fog nozzle, are located at convenient points on the three floors. A two inch pipe supplies each hose from a three inch header. One and one-half inch valves at each cabinet control the flow to each hose. Each hose is connected and ready for use. Dry chemical and carbon dioxide extinguishers are available at strategic locations. The combination of wet and dry units allows a choice of equipment for the size and type of fire.

The possibility of a large fire is remote because the building is constructed of concrete and only a small amount of combustible materials are used or stored. Either the wet or dry equipment is adequate for protection. The two systems provide added safety and equipment selection.

\subsubsection{TURBINE DECK AND AUXILIARY BAY STRUCTURE}

The turbine deck and auxiliary bay structure is protected by the following combination of sprinklers, hose reels and portable units.

a. Hose reels, each with 50 feet of lined one and one-half inch cotton hose and a brass adjustable stream or fog nozzle.

b. Twenty pound portable dry chemical units.

c. Thirty pound portable dry chemical unit.

d. One hundred fifty pound portable dry chemical units.

e. Spray sprinklers for the hydrogen seal oil unit and the lube oil reservoir area.

The sprinklers provide a water spray from all sides of the equipment at the same time. This action is effective because it cools, smothers and floods burning liquids. The hose reels are located so that all areas of the plant can be reached. The fast acting, easy to handle portable units, located 


\subsubsection{TURBINE DECK AND AUXILIARY BAY STRUCTURE (Continued)}

at key points throughout the plant, will control most fires but are most effective on electrical and small trash fires.

\subsubsection{CONTAINMENT SPHERE}

Containment sphere protection equipment consists of two water spray loops, 20-pound dry chemical units and 15-pound carbon dioxide units. The chemical and dry units are located at points of maximum possible use and availability.

The refueling water pump supplies the two water spray loops with borated water from the refueling water storage tank. The use of borated water at refueling concentrations for fire protection inside the sphere prevents any inadvertent addition of reactivity to the Reactor Coolant System.

A remotely operated valve is provided in each of the two spray headers. The remote valves and the refueling water pump are manually controlled from the Control Room.

The principal combustible materials in the Sphere are insulating materials on electrical motors and wiring and a limited amount of lube oil in the three reactor coolant pump motors. The motors are equipped with bearing thermo-couples, wired to alarms in the control room. The thermo-couples are actuated on rising temperatures in the motor bearings. The containment air conditioning systems are equipped with temperature elements which will actuate an alarm in the Control Room in the event of excessive temperature. The alarms alert operating personnel to the possibility of fire.

\subsubsection{WAREHOUSE}

The warehouse is protected by hose reels, each with 50 feet of one and onehalf inch, lined cotton hose and a fog nozzle. The hose reels are placed so that a11 parts of the building are covered. To provide additional coverage, 15-pound carbon-dioxide portable units are placed in strategic locations throughout the warehouse. The paint storage room in the warehouse is protected by a carbon-dioxide unit. This combination of equipment will control the varied types of fires that could occur.

\section{5 .8 VENTILATING EQUTPMENT ROOM}

The building housing the ventilating equipment is equipped with a 15-pound carbon-dioxide-unit. This extinguisher should control any oil, electrical, or other type fire that may occur in this area.

\subsubsection{REACTOR AUXILIARY BUILDING}

The reactor auxiliary building is protected by portable dry chemical units and hose reels. The possibility of fire in this building is slight since 


\subsubsection{REACTOR AUXILIARY BUILDING (Continued)}

there are few combustible materials inside. Such materials consist of the insulation on motors and wires and the lubricating oils in the motors. The extinguishers selected are effective for oil and electrical fires. 


\section{SECTION 12 \\ CHEMICAL REACTIONS}

\subsection{CHEMICAL REACTIONS}

\section{1 .1 GENERAL}

Exothermic chemical reactions between commonly used fuel cladding metals (principally zircaloy) and a steam atmosphere have been shown to occur under conditions possible in the event of a major loss-of-coolant accident. Although the initial core cladding material is stainless steel, it is expected that future cores will employ zircaloy cladding. Consequently, the plant systems and Containment Sphere have been designed to accommodate entire cores clad with zircaloy.

\subsubsection{ZIRCONIUM-WATER REACTIONS}

The zirconium-water reaction is described by the chemical equation:

$$
\mathrm{Zr}+2 \mathrm{H}_{2} \mathrm{O} \rightarrow \mathrm{ZrO}_{2}+2 \mathrm{H}_{2}+\text { heat }
$$

The amount of heat liberated in this reaction is approximately 2,800 Btu per pound of reacting zirconium. The heat release in calories-per-grammole of solid zirconium reacted as a function of absolute reaction temperature on the Kelvin scale is given by the expression ${ }^{1}$,

$$
\Delta \mathrm{H}=-149,120+8.5 \mathrm{~T}-1.44 \times 10^{-3} \mathrm{~T}^{2}+\frac{2.41 \times 10^{5}}{\mathrm{~T}}
$$

For reaction of molten zirconium, the heat of fusion, given as 5,500 calories-per-gram-mole ${ }^{1}(9,900$ Btu per pound) must also be added.

The hydrogen produced in the reaction is evolved at temperatures much greater than the ignition temperature, and burns as it comes in contact with the air. The energy release from the hydrogen combustion equivalent may be as much as an additional 2,150 Btu per pound of zirconium reacted. For purposes of sphere analysis, the hydrogen is assumed to be immediately released from the reactor coolant system as soon as it is generated.

\subsubsection{PARABOLIC RATE EQUATION}

Following a loss-of-coolant accident in which the core is exposed to a steam atmosphere, zirconium undergoes a corrosion-type reaction with steam. The reaction achieves significant rates only after the cladding has been heated by residual heat generation to temperatures in excess of $1,800 \mathrm{~F}$. The rate of reaction is dependent upon the availability of steam, the thickness of the zirconium oxide layer developed at the cladding surface, and the temperature of the metal exposed to the steam. The following expression, known as the parabolic rate equation, relates weight of 


\subsection{PARABOLIC RATE EQUATION (Continued)}

zirconium reacted to time and temperature, when an excess of steam is available:

$$
\mathrm{W}^{2}=33.3 \times 10^{6}(\mathrm{t}) \exp -\left(\frac{45,500}{\mathrm{RT}}\right)
$$

where

$$
\begin{aligned}
& \mathrm{W}=\text { weight of metal reacted, } \mathrm{mg} / \mathrm{cm}^{2} \\
& \mathrm{t}=\text { time, seconds } \\
& \mathrm{R}=\text { universal gas constant, } 1,986 \text { calories per mole, } \mathrm{K} \\
& \mathrm{T}=\text { absolute temperature, } \mathrm{K}
\end{aligned}
$$

This equation has been varified in several independent tests. The constants used were evaluated by Baker and Just from experiemental findings of several investigators ${ }^{2}$.

\subsubsection{STAINLESS STEEL REACTIONS}

Stainless steel reactions with steam are less significant than zirconiumsteam reactions, with regard to both rate and quantity of energy release to the sphere. The stainless steel reaction liberates between 259 and 455 Btu per pound of metal reacting ${ }^{3}$. Excluding the energy of hydrogen-oxygen recombination the stainless steel reaction releases less than one-fifth of the energy of the zirconium-water reaction. Thus, the effect of reaction on the rate of rise of cladding temperature is less important with stainless steel. Also, stainless steel reacts much more slowly than zirconium. The extent of reaction has been observed to be significant only at or above the melting point of approximately $2,500 \mathrm{~F}$.

Therefore, the total amount of stainless steel reaction over the periods of time that are of interest will be much less than for zirconium. Less cooling is required to stop the reaction, since the energy released from the reaction of stainless steel is not regenerative to the extent of the zirconium-water reaction. Consequently, the metal-water reaction analyses have been based upon the use of zircaloy cladding.

\subsubsection{ZIRCONIUM-WATER REACTION ENERGY RELEASE CALCULATIONS}

Evaluation of the energy release associated with the zirconium-water reaction required application of the parabolic rate equation considering the transient rise in temperature of the core following the loss-of-coolant accident. The availability of steam to continue the reaction is also considered in evaluating the reaction energy release. The boil-off of water remaining below the core after the initial blowdown is governed by conduction of heat from the lower reactor vessel structure and the thermal and gamma radiation heating from the core. The rate of reaction is limited by the parabolic rate law early in the transient where an excess of steam is 


\subsubsection{ZIRCONIUM-WATER REACTION ENERGY RELEASE CALCULATIONS (Continued)}

available and cladding temperatures are low. The reaction rate is limited by the rate of steam evolution later in the transient, when cladding temperatures are high, and when the parabolic rate equation indicates a rate of reaction higher than the rate at which steam can be generated to support the reaction.

\subsubsection{LOCAL TRANSIENT CALCULATIONS}

Where an excess of steam is available, a digital computer code has been used to solve the controlling equations for local points in the core. Overal1 core conditions are determined by dividing the core into several volumes, each of which is represented by an average initial power level

expressed as a fraction of the peak heat flux. The local transient calculation is performed for each value of initial heat flux. The total zirconiumwater reaction energy is the sum of the energy release transients determined for each fraction of core volume.

\section{Heat Transfer Mode 1}

The starting point for the calculation of the local cladding and fuel temperature transients is the initiation of the loss-of-coolant accident. The calculation is based on a heat balance which considers heat transfer between fuel pellet and cladding as well as heat transfer between the cladding surface and coolant fluid. A lumped parameter technique is employed for a unit length of fuel rod assuming no axial heat conduction. The heat transfer model utilizes three nodes in the fuel pellet and one node to represent the cladding. Physical properties (e.g., thermal conductivity, specific heat of the fuel, cladding, and oxide layer) are varied as a function of temperature. The model accounts for the thermal energy initially stored in the fuel at the beginning of the transient, the residual heat generation in the fuel during the transient, and the heat of reaction in the cladding based upon the parabolic rate equation.

\section{Calculation Assumptions}

Conservative assumptions which follow are employed throughout the calculation:

a) During the initial phases of blowdown, a cladding surface, heat transfer coefficient of 1,000 Btu per hour per degree Farenheit per square foot is used. This heat transfer coefficient is approximately one-third of the value for normal operation and is assumed to drop to zero once the surface of reactor coolant falls below the core. This assumption of no heat transfer from the cladding surface is then used for the entire period of the transient until the reaction is halted when safety injection causes water to rise into the core. The resumption of cooling at this point causes cladding surface temperature to fall rapidly and halts the metalwater reactions.

b) The hydrogen evolved is assumed to present no barrier for diffusion of the steam reactant to the cladding surface. 


\subsubsection{LOCAL TRANSIENT CALCULATIONS (Continued)}

c) In the event the cladding temperature exceeds the melting point of zirconium, the zirconium oxide layer is assumed to prevent the molten cladding from flowing into cooler regions where the reaction could stop due to a lowering of temperature. Under this assumption, the reaction continues at the rate determined by the parabolic rate equation.

d) Should the cladding temperature exceed the melting temperature of the oxide (approximately 4,800 F), the cladding is assumed to run off and drop into any water remaining in the bottom of the reactor vessel. An additional 10 percent of this metal will react on contact with the water.

e) If the rate of steam evolution from heat transferred to the water below the core is equal to or less than the consumption rate determined by the parabolic rate equation, the assumption is made that all the steam evolved takes part in the reaction.

\subsubsection{RESULTING ZIRCONIUM-WATER REACTION ENERGY}

Results of zirconium-water reaction calculations for a loss-of-coolant accident with a core containing 272 pound-moles of zirconium are shown in Figure 12.1. Representive core parameters are as follows:

$\begin{array}{ll}\text { Number of fuel rods } & 28,260 \\ \text { Length of fuel rod } & 10 \mathrm{ft} \\ \text { Cladding oD } & 0.422 \mathrm{in} . \\ \text { Cladding thickness } & 0.0243 \mathrm{in} . \\ \text { Fuel pellet diameter } & 0.3669 \mathrm{in} . \\ \text { Reactor power } & 1,347 \mathrm{Mwt}\end{array}$

Curve $A$ in Figure 12.1 shows the results of the energy release calculations over the period of interest before the reaction is terminated by safety injection. There is no appreciable reaction during the first 20 seconds following the hypothetical pipe rupture. This period elapses before the hottest portions of the cladding are heated significantly above the reaction threshold temperature by residual heat generation. Initially, the reaction rate is determined by the parabolic rate equation. The boil-off rate of water remaining below the core in the reactor vessel exceeds requirements at this time. As larger portions of the core are heated above the reaction threshold, the reaction proceeds at a rate limited by the availability of steam. When the reaction is terminated by water rising above the bottom of the core ( 64 seconds after the break as defined in Paragraph 10.1), a maximum of 2.4 percent of clad has reacted, adding approximately 1.6 million Btu of reaction energy to the residual heat stored in the core, and liberating approximately 1.3 million Btu directly to the sphere from combustion of the evolved hydrogen. 


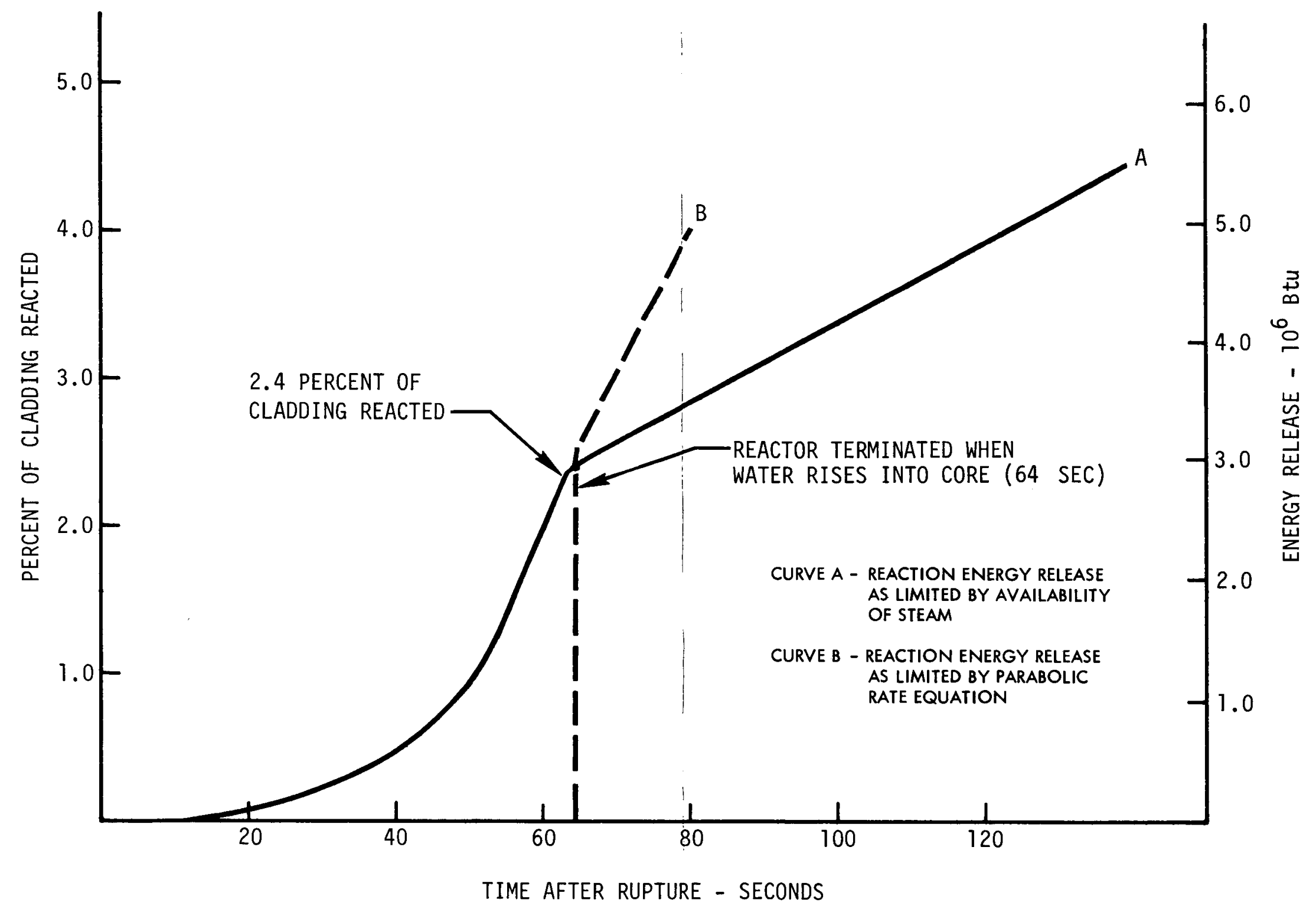

FIGURE 12.1

ZIRCONIUM-WATER AND HYDROGEN

COMBUSTION ENERGY RELEASE

VERSUS TIME AFTER RUPTURE 


\subsubsection{RESULTING ZIRCONIUM-WATER REACTION ENERGY (Continued)}

Curve $B$ in Figure 12.1 is an extension of the energy release curve, assuming an excess of steam where the reaction rate is limited only by the parabolic rate equation.

It should be noted that the 64-second period prior to termination of the reaction is based upon the following assumptions of safety injection system performance:

a. One safety injection train is functioning and one of the three injection lines spills its flow.

b. The reactor vessel has no water remaining after the initial blowdown (a conservative assumption, considering that the reaction calculation is based on the existence of water below the core, which is heated to form the vapor necessary to sustain the reaction).

In the more likely event that all three valves open, both pumping trains function, and one line delivers ineffectively into the ruptured loop, the refilling time is reduced from 64 seconds to 46 seconds for the hypothetical break. A corresponding reduction in total reaction to less than 0.7 percent of the cladding would be obtained.

\subsubsection{ZIRCONIUM-WATER REACTION ENERGY USED FOR CONTAINMENT SPHERE EVALUATION}

The sphere pressure transient analyses given in the Containment Sphere Design Report were based upon earlier simplified analyses with much reliance on hand calculations. It contains a degree of conservatism believed excessive in comparison with the more realistic method described above. The energy release transient was calculated using the LOCTA digital code. The code numerically solves heat balance equations for the fuel rod, considering no metal-to-water reaction. The zirconium-water reaction energy release was superimposed upon the base-line temperature transient, using a step-by-step hand calculation and the parabolic rate equation to determine the cladding temperature and the amount of metal reacted as a function of time.

This simplified calculation differs from the more realistic calculation in the following ways:

a) A simpler model was used to facilitate hand calculations. This model assumed all energy from the zirconium-water reaction to be stored in the cladding, without taking advantage of heat transfer to the cooler fuel pellet. The assumption of no heat transfer from cladding to pellet results in a cladding temperature much higher than the adjacent fuel pellet, and in unrealistically high reaction rates.

b) The zirconium oxide layer was assumed to break up when the meta1 melting temperature of $3,375 \mathrm{~F}$ is reached. This was handled in the calculation by the assumption that the metal cladding is continuously exposed at the melting temperature without oxide layer protection until it is consumed. 


\subsubsection{ZIRCONIUM-WATER REACTION ENERGY USED FOR CONTAINMENT SPHERE EVALUATION (Continued)}

Tests have shown the oxide film to be very tenacious. Furthermore, without oxide layer support, the molten metal would not remain in place and would drop to a region of lower temperature.

c) No consideration was given to the limited availability of steam.

d) On1y 50 percent of the cladding was assumed available for reaction, compared with 100 percent in the more realistic calculation.

Figure 12.2 shows the sum of residual heat and reaction energy as a function of time after system rupture. The time of reaction has been arbitrarily extended to 320 seconds. Hydrogen combustion energy is included.

Curve $A$, the result of the more realistic calculation, corresponds to Curve A of Figure 12.1. Curve B is the result of the simplified calculation just described. Comparing the curves, it can be seen that the simplified method used in the sphere analyses results in a metal-water and hydrogen-oxygen reaction energy of release of $44 \times 10^{6} \mathrm{Btu}$, at 320 seconds. This is more than double the energy calculated by the more realistic method. Thus, the design basis for analysing the effect of metal-water reaction on sphere system pressure leads to very conservative conditions.

\subsubsection{REFERENCES}

1. B. Lustman, "Zirconium-Water Reaction Data and Application to PWR Loss-of-Coolant Accident," WAPD-SC-543, May 1957.

2. L. Baker and L. Just, "Studies of Metal-Water Reactions at High Temperatures, III Experimental and Theoretical Studies of the Zirconium-Water Reaction," ANL-6548, Argonne National Laboratory, May 1962 .

3. W. B. Cottrell and A. W. Savolainen, "U.S. Reactor Containment Technology," ORNL Central File No. 64-11-31 (Draft). 


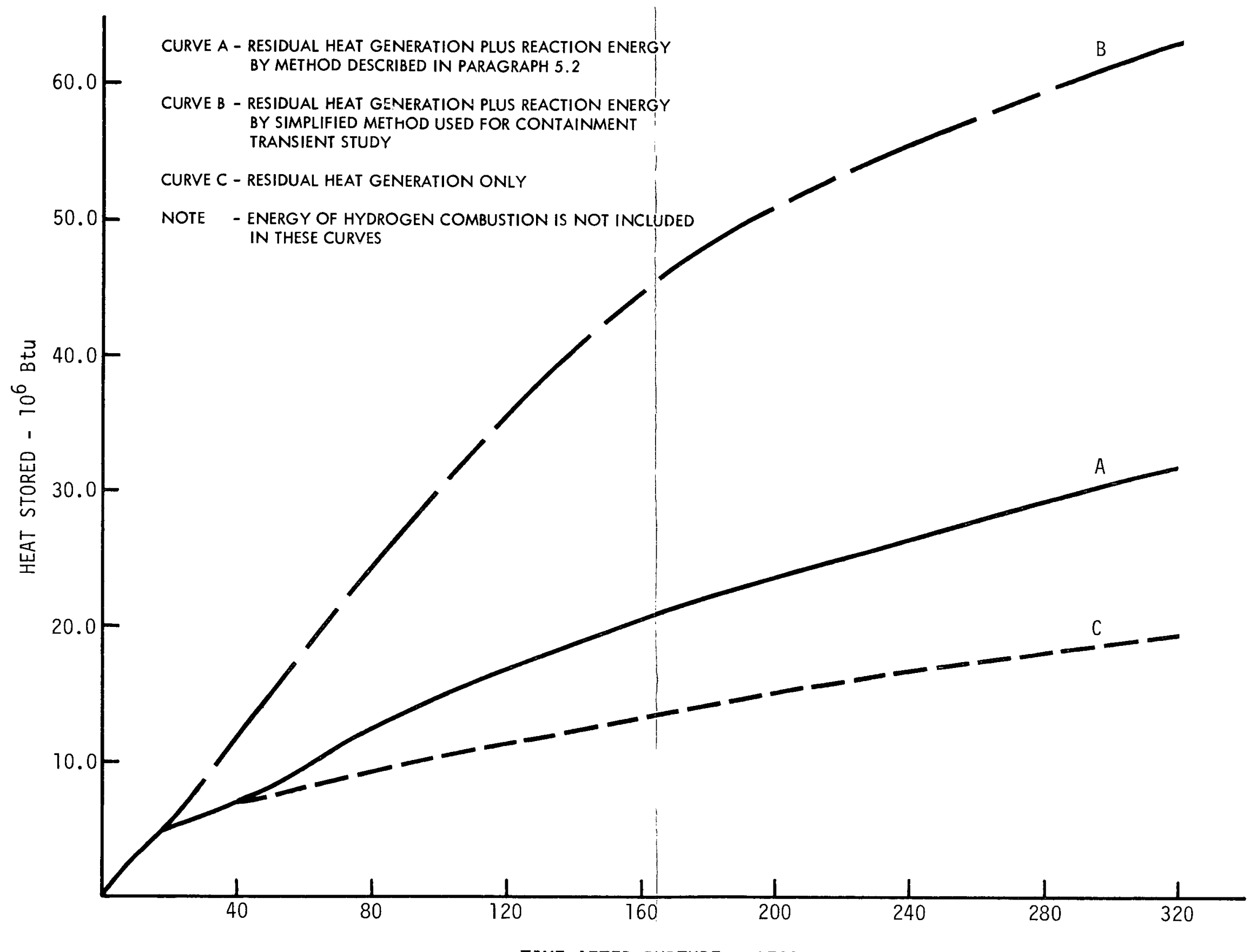

TIME AFTER RUPTURE - SECONDS

FIGURE 12.2

HEAT GENERATED VERSUS

TIME AFTER RUPTURE 


\section{SECTION 13 \\ FISSION PRODUCT INVENTORY}

\subsection{FISSION PRODUCT RELEASE FROM FUEL}

\subsubsection{INTRODUCTION}

As a basis for the calculation of potential radiation dosage from various accidents, this section defines the quantities of fission product and other radioactive materials subject to release under the conditions appropriate to the analysis.

The following sources have been considered:

\section{Source Material Related Analysis $\quad \underline{\text { Reference }}$}

Core Fission

Products

Reactor Coolant Activity

Auxiliary Systems Activity

Spent Fuel Activity

\section{Rod ejection (1imited fuel damage)} Loss of coolant (limited fuel damage), Hypothetical core meltdown

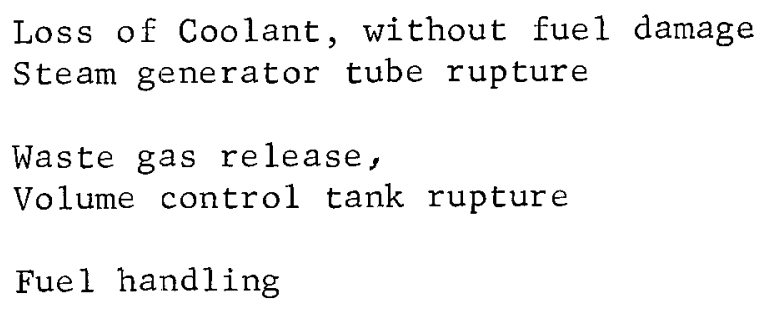

Fuel handling

Paragraph $13 \cdot 1.2$

Paragraph 13.1 .3

Paragraph 13.1.4

Paragraph 13.1 .5

The effect of plant features which limit the extent of the release (for example, the fraction of the core which undergoes a given type of damage) is discussed in the section of this report dealing with the particular accident or malfunction assumed to cause the release.

\subsubsection{CORE FISSION PRODUCTS}

The buildup of core fission products is calculated with the digital code FIPCO-2, programmed for the IBM-7094 computer. The code numerically solves the following differential equations of production and removal for a11 significant mass-number chains:

For parent nuclides in the pellet,

$$
\frac{\mathrm{dN}_{\mathrm{p} i}}{\mathrm{dt}}=\mathrm{F} \mathrm{y}_{i} \mathrm{P}-\left(\lambda_{i}+\nu_{i}+\phi \sigma_{i}\right) \mathrm{N}_{\mathrm{pi}}
$$




\subsubsection{CORE FISSION PRODUCTS (Continued)}

For daughter nuclides in the pellet,

$$
\frac{d N}{d t}=F y_{j} P+\lambda_{i} N_{p i}-\left(\lambda_{j}+\nu_{j}+\phi \sigma_{j}\right) N_{p j}
$$

For parent nuclides in the gap,

$$
\frac{d N_{g i}}{d t}=v_{i} N_{p i}-\left(\lambda_{i}+\phi \sigma_{i}\right) N_{g i}
$$

For daughter nuclides in the gap,

$$
\frac{d N_{g j}}{d t}=\nu_{j} N_{p j}+\lambda_{i} N_{g i}-\left(\lambda_{j}+\phi \sigma_{j}\right) N_{g j}
$$

where

$$
\begin{aligned}
& F=\text { fission rate per megawatt-second thermal } \\
& N=\text { population of nuclide } \\
& P=\text { power, level, megawatts thermal } \\
& t=\text { irradiation time, sec. } \\
& y=\text { fission yield, atoms per fission } \\
& \lambda=\text { radioactive decay constant, sec } \mathrm{s}^{-1} \\
& \nu=\text { escape coefficient for diffusion out of } \mathrm{UO}_{2}, \mathrm{sec}^{-1} \\
& \varnothing=\text { thermal neutron flux, neutrons } / \mathrm{cm}^{2}-\mathrm{sec} \\
& \sigma=\text { thermal neutron capture cross section, } \mathrm{cm}^{2} \\
& g \quad \text { refers to pellet-clad gap } \\
& i \quad \text { refers to parent nuclide } \\
& j \quad \text { refers to daughter nuclide } \\
& \mathrm{p} \quad \text { refers to fuel pellets }
\end{aligned}
$$

By applying proper boundary conditions, the computation is made for the separate power histories of the three core regions, and irradiation time is selected to give the maximum aggregate fission product inventory. 


\subsubsection{CORE FISSION PRODUCTS (Continued)}

\section{Isotope Grouping}

For use in the analysis of the hypothetical core melt-down accident, the isotopes are grouped according to their relative tendency to be released from the core at elevated temperature. The following classification and release fractions are used:

a. From any fuel rod in which the peak clad temperature exceeds the assumed failure limit, all noble gases and halogens present in the pellet-clad gap are assumed released.

b. From any pellet whose average temperature exceeds 2,500 F, the TID-14844 release fractions are assumed. (The TID-14844 release fractions are: 100 percent noble gases, 50 percent halogens, and one percent remaining species.)

\section{Fission Product Calculation}

Tables 13.1 and 13.2 present results of the aggregate core fission product calculation. For consideration of direct gamma radiation, Table 13.1 presents the source strength of those fission products subject to release from the fuel pellets and from the pellet-clad gap, respectively. Source strength is expressed in Mev/sec for each of five photon energy groups, computed on a megawatt thermal basis for the portion of the core exceeding the limits described.

Inhalation hazard following a fission product release is primarily due to radioactive iodine. Table 13.2 gives the curie inventories of the iodine isotopes subject to release from the fuel pellets and from the pellet-clad gap, respectively. Like the direct gamma sources, these data are also given on a megawatt thermal basis for the involved portions of the core.

To calculate the source release in a case where only a part of the core exceeds the pellet release criteria, the distribution of core power as a function of core volume is employed. The gross fission product distribution follows that of the thermal power. In Figure 13.1 the average thermal power of the hottest volume fraction $\emptyset_{\mathrm{V}}$, expressed as a fraction of equilibrium core power, is plotted as a function of $\emptyset_{\mathrm{V}}$.

In a given accident case, the core volume increment reaching a given temperature is determined by the method described in Section 12, Chemical Reactions. From this thermal power increment $\left(\emptyset_{\mathrm{V}}\right)$ the fraction of equilibrium core power is obtained from Figure 13.1. The product of the fraction of equilibrium core power, the gross thermal power of the core ( 1,347 Mwt), and the values of the fuel pellet fission products give the total pellet release. The resultant product when added to the entire core pellet-clad gap activity gives the total fission product release from the core. All pellet-clad gap activity from the entire core will be released within the time interval for the average temperature of any pellet to reach 2,500 $\mathrm{F}$. 
Table 13.1 Direct Radiation Sources Following Fuel Meltdown

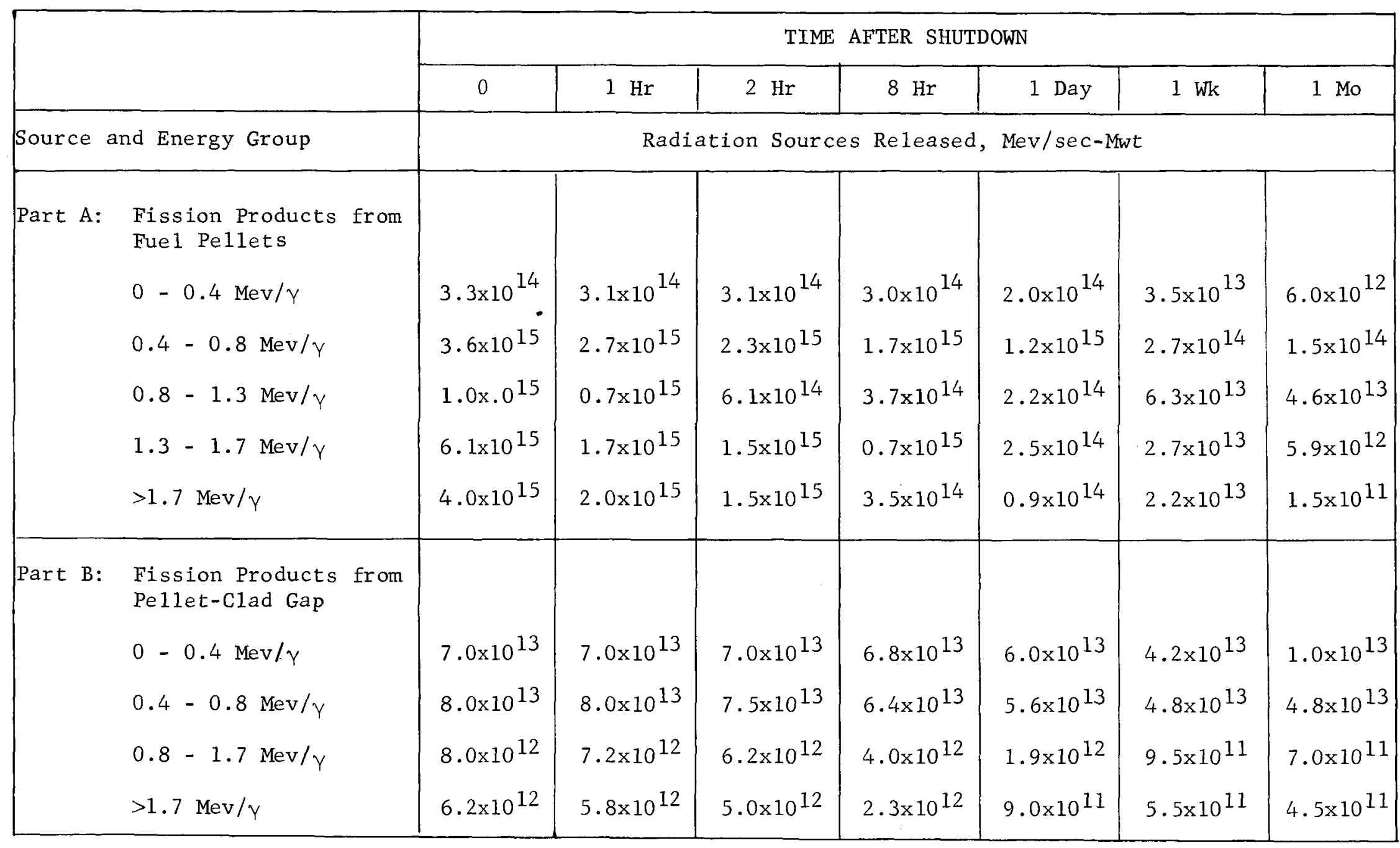


Table 13.2 Radioactive Iodine Sources Following Fuel Melting

\begin{tabular}{|c|c|c|c|c|c|c|c|c|}
\hline \multirow{2}{*}{\multicolumn{2}{|c|}{$\begin{array}{l}\text { Source and } \\
\text { Release Fraction }\end{array}$}} & \multicolumn{7}{|c|}{ Time After Shutdown } \\
\hline & & 0 & $1 \mathrm{Hr}$ & $2 \mathrm{Hr}$ & $8 \mathrm{Hr}$ & 1 Day & $1 \mathrm{Wk}$ & 1 Mo \\
\hline \multirow{6}{*}{$\begin{array}{l}\text { Part A: } 50 \% \\
\text { Iodine from } \\
\text { Fuel pellets }\end{array}$} & Isotope & \multicolumn{7}{|c|}{ Iodine Released, Curies/Mwt } \\
\hline & I-131 & $1.3 \times 10^{4}$ & $1.3 \times 10^{4}$ & $1.3 \times 10^{4}$ & $1.2 \times 10^{4}$ & $1.1 \times 10^{4}$ & $6.7 \times 10^{3}$ & $3.0 \times 10^{2}$ \\
\hline & I- 132 & $1.9 \times 10^{4}$ & $1.4 \times 10^{4}$ & $1.1 \times 10^{4}$ & $1.7 \times 10^{3}$ & $1.3 \times 10^{1}$ & - & - \\
\hline & I-133 & $2.8 \times 10^{4}$ & $2.8 \times 10^{4}$ & $2.7 \times 10^{4}$ & $2.2 \times 10^{4}$ & $1.3 \times 10^{4}$ & $1.1 \times 10^{2}$ & - \\
\hline & I-134 & $3.4 \times 10^{4}$ & $1.6 \times 10^{4}$ & $7.2 \times 10^{3}$ & $6.2 \times 10^{1}$ & - & - & - \\
\hline & I-135 & $2.6 \times 10^{4}$ & $2.4 \times 10^{4}$ & $2.1 \times 10^{4}$ & $1.1 \times 10^{4}$ & $2.2 \times 10^{4}$ & - & - \\
\hline \multirow{5}{*}{$\begin{array}{l}\text { Part B: } 100 \% \\
\text { Iodine from } \\
\text { Pellet-Clad Gap }\end{array}$} & $I-131$ & $3.2 \times 10^{2}$ & $3.2 \times 10^{2}$ & $3.2 \times 10^{2}$ & $3.0 \times 10^{2}$ & $2.7 \times 10^{2}$ & $1.7 \times 10^{2}$ & $0.7 \times 10^{1}$ \\
\hline & I- 132 & $2.0 \times 10^{2}$ & $1.5 \times 10^{2}$ & $1.2 \times 10^{2}$ & $2.5 \times 10^{1}$ & 5.1 & 1.4 & - \\
\hline & I-133 & $7.7 \times 10^{1}$ & $7.7 \times 10^{1}$ & $7.5 \times 10^{1}$ & $6.1 \times 10^{1}$ & $3.6 \times 10^{1}$ & - & - \\
\hline & $I-134$ & 4.1 & 1.9 & - & - & - & - & - \\
\hline & I- 135 & $2.3 \times 10^{1}$ & $2.1 \times 10^{1}$ & $1.9 \times 10^{1}$ & $1.0 \times 10^{1}$ & - & - & - \\
\hline
\end{tabular}




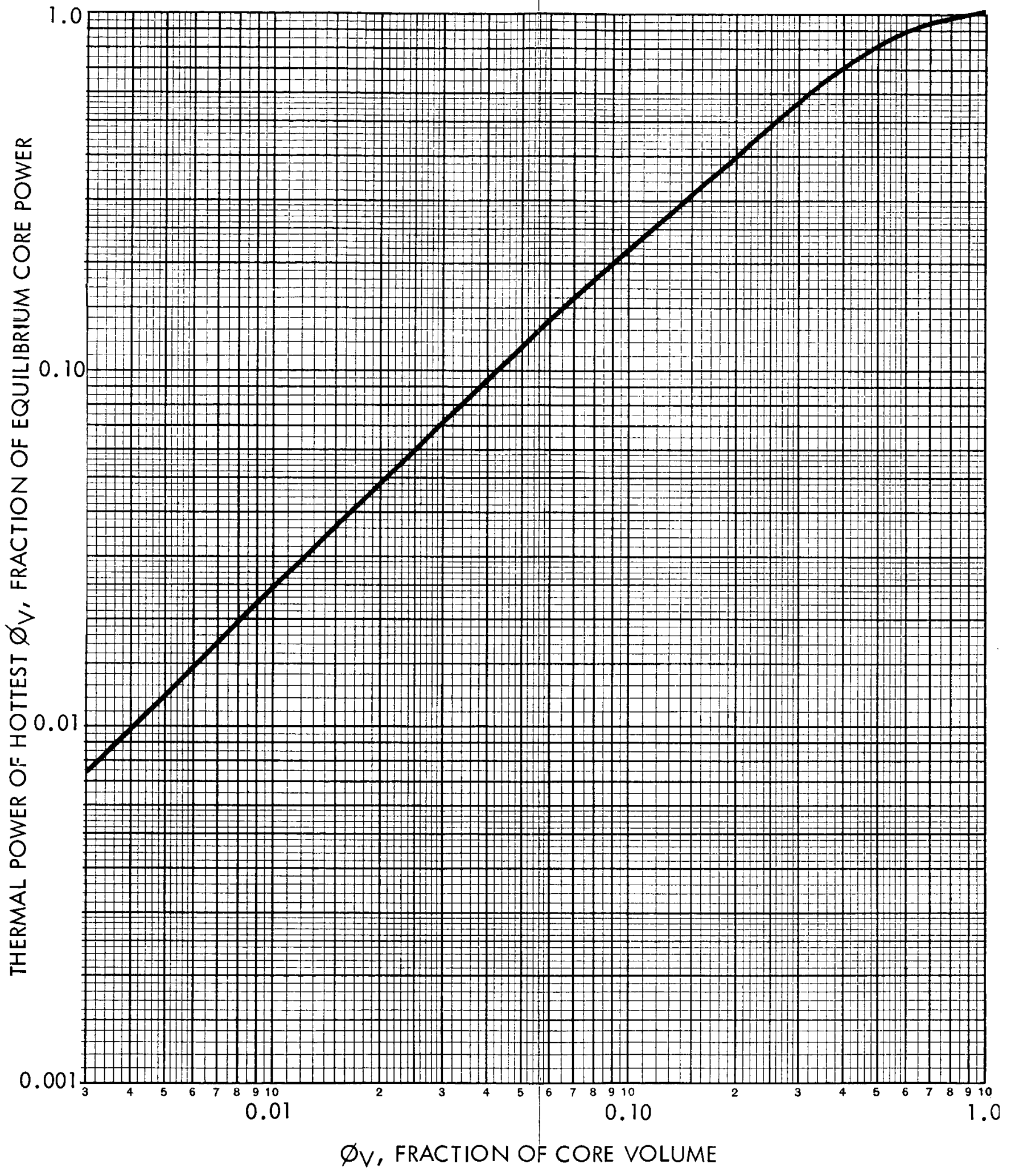

FIGURE 13.1 DISTRIBUTION OF THERMAL POWER AS A FUNCTION OF CORE VOLUME 


\subsubsection{REACTOR COOLANT ACTIVITY}

The inventory of radioactive material in the reactor coolant may be divided into three classes: fission products, activated corrosion products, and products of the activation of water and dissolved air. Only the first two are significant as accident sources, since the products of coolant and dissolved air activation decay within a few seconds to levels which are quite low in comparison with the others.

The fission product content of the reactor coolant is computed with the digital code FIPCO-2 described in Paragraph 13.1.2. For this result, the code makes use of the following differential equations:

For parent nuclides in the coolant,

$$
\frac{d N}{d t}=D v_{i} N_{p i}-\left(\lambda_{i}+R \eta_{i}+\frac{B^{\prime}}{B_{0}-t B^{\prime}}\right) N_{w i}
$$

For daughter nuclides in the coolant,

$$
\frac{d N}{d t}=D v_{j} N_{p j}-\left({ }^{\lambda} j+R \eta_{j}+\frac{B^{\prime}}{B_{o}-t B^{\prime}}\right) N_{w j}+N_{i} N_{w i}
$$

where

$$
\begin{aligned}
& \mathrm{D}=\text { fraction of fuel rods having defective cladding } \\
& \mathrm{R}=\text { purification flow, coolant system volumes per sec. } \\
& \mathrm{B}_{\mathrm{O}}=\text { initial boron concentration, ppm } \\
& \mathrm{B}^{\prime}=\text { boron concentration reduction rate by feed and bleed, ppm per sec } \\
& \eta=\text { removal efficiency of purification cycle for nuclide } \\
& \mathrm{W}=\text { refers to coolant }
\end{aligned}
$$

\section{Boundary Conditions}

Boundary conditions satisfied by the numerical solution include the following effects :

a. Use of the purification demineralizer at a constant volumetric flow rate, with an assumed removal factor of 10 for all isotopes except noble gases, yttrium, molybdenum, and cesium for which no removal effect is assumed. 


\subsubsection{REACTOR COOLANT ACTIVITY (Continued)}

b. Use of feed and bleed to the radwaste disposal system at a variable volumetric rate, as required to reduce boron concentration at a rate of $4 \mathrm{ppm}$ per day,

c. Degassing and dilution of the reactor coolant with the refueling water at each refueling shutdown.

d. Keactor shutdown, with no fission yield or neutron activation, during the appropriate phase of each core cycle.

\section{Corrosion Product Activities}

Corrosion product activities are calculated by the digital code CORA. This code computes the concentration of active nuclides formed by neutron irradiation and subsequent exchange of core-deposited crud with that in suspension. Removal by purification, deposition, and decay are taken into account.

Tables 13.3 and 13.4 give the maximum concentrations and total system inventories of the significant fission and corrosion products, expressed in terms of isotopic quantities and as photon energy groups for direct gamma source application. The table is based on a maximum power rating of 1347 Mwty and on a reference defective fuel rod population of one per cent.

\subsubsection{AUXILIARY SYSTEMS ACTIVITY}

\section{Volume Control Tank}

In the volume control tank, maximum activity level occurs when the chemical and volume control system is operating with the purification demineralizer bypassed. In this case, the contained source consists of reactor coolant activity essentially equal to that 1 isted in Table 13.3 on a mass basis, but greater by a factor of 1.3 on a volumetric basis due to the density change. The vapor space of the volume control tank contains gaseous fission products in equilibrium with the entering reactor coolant. The specific activity of the vapor phase is calculated using Henry's Law constants for the volatile fission products (noble gases and halogens).

The resulting liquid and vapor phase activities are listed in Table 13.5 for the reference condition of one-percent fuel defects, 1,347 Mwt and the minimum steady-state liquid volume in the tank (to yield a maximum volatile fission product source).

\section{Waste Gas Decay Tanks}

The other auxiliary system location in which it is possib1e to accumulate a significant quantity of radioactive material with a potential for release is the waste gas decay tanks.

The maximum inventory of the waste gas decay tanks is calculated by assuming the reactor undergoes a cold shutdown at the point in core life when 
Table 13.3 Maximum Fission and Corrosion Product Isotopic Inventories of Reactor Coolant for Reference One Percent Fuel Defect Case

(Based upon $570 \mathrm{~F}$ Coolant)

\begin{tabular}{|c|c|c|}
\hline Isotope & $\begin{array}{l}\text { Specific Activity } \\
\text { Microcuries/cc }\end{array}$ & $\begin{array}{l}\text { Coolant } \\
\text { Inventory, } \\
\text { Curies }\end{array}$ \\
\hline $\begin{array}{l}\mathrm{Br}-84 \\
\mathrm{Kr}-85 \\
\mathrm{Kr}-85 \quad(\mathrm{~m}) \\
\mathrm{Kr}-87 \\
\mathrm{Kr}-88 \\
\mathrm{Rb}-88 \\
\mathrm{Rb}-89 \\
\mathrm{Sr}-89 \\
\mathrm{Sr}-90 \\
\mathrm{Y}-90 \\
\mathrm{Sr}-91 \\
\mathrm{Y}-91 \\
\mathrm{Mo}-99 \\
\mathrm{Te}-129 \\
\mathrm{I}-129 \\
\mathrm{I}-131 \\
\mathrm{Te}-132 \\
\mathrm{I}-132 \\
\mathrm{I}-133 \\
\mathrm{Xe}-133 \\
\mathrm{Te}-134 \\
\mathrm{I}-134 \\
\mathrm{Cs}-134 \\
\mathrm{I}-135 \\
\mathrm{Xe}-135 \\
\mathrm{Cs}-136 \\
\mathrm{Cs}-137 \\
\mathrm{Xe}-138 \\
\mathrm{Cs}-138 \\
\mathrm{Ba}-140 \\
\mathrm{~L}-140 \\
\mathrm{Co}-60 \\
\mathrm{Fe}-59 \\
\mathrm{Co}-58 \\
\mathrm{Mn}-56 \\
\mathrm{Mn}-54\end{array}$ & $\begin{array}{c}0.16 \\
4.2 \\
1.3 \\
0.73 \\
2.1 \\
2.1 \\
5.0 \times 10^{-2} \\
2.0 \times 10^{-3} \\
1.0 \times 10^{-4} \\
1.9 \times 10^{-4} \\
1.0 \times 10^{-3} \\
2.8 \times 10^{-2} \\
2.5 \\
1.6 \times 10^{-2} \\
2.4 \times 10^{-8} \\
1.4 \\
0.13 \\
0.50 \\
1.9 \\
158 \\
1.4 \times 10^{-2} \\
0.26 \\
0.68 \\
0.99 \\
4.4 \\
7.0 \times 10^{-2} \\
15.7 \\
0.32 \\
0.52 \\
2.1 \times 10^{-3} \\
7.1 \times 10^{-4} \\
1.5 \times 10^{-3} \\
1.9 \times 10^{-3} \\
8.5 \times 10^{-3} \\
2.3 \times 10^{-2} \\
4.4 \times 10^{-3}\end{array}$ & $\begin{array}{l}31 \\
800 \\
250 \\
140 \\
400 \\
400 \\
10 \\
0.4 \\
0.02 \\
0.04 \\
0.2 \\
5.3 \\
477 \\
3 \\
0.5 \\
5 \times 10 \\
267 \\
25 \\
95 \\
363 \\
30,200 \\
2.7 \\
50 \\
130 \\
189 \\
840 \\
13 \\
3000 \\
61 \\
100 \\
0.4 \\
0.1 \\
0.29 \\
0.36 \\
1.6 \\
4.4 \\
0.84\end{array}$ \\
\hline
\end{tabular}


Table 13.4 Maximum Fission and Corrosion Product Gamma Sources of Reactor Coolant for Reference One Percent Fuel Defect Case

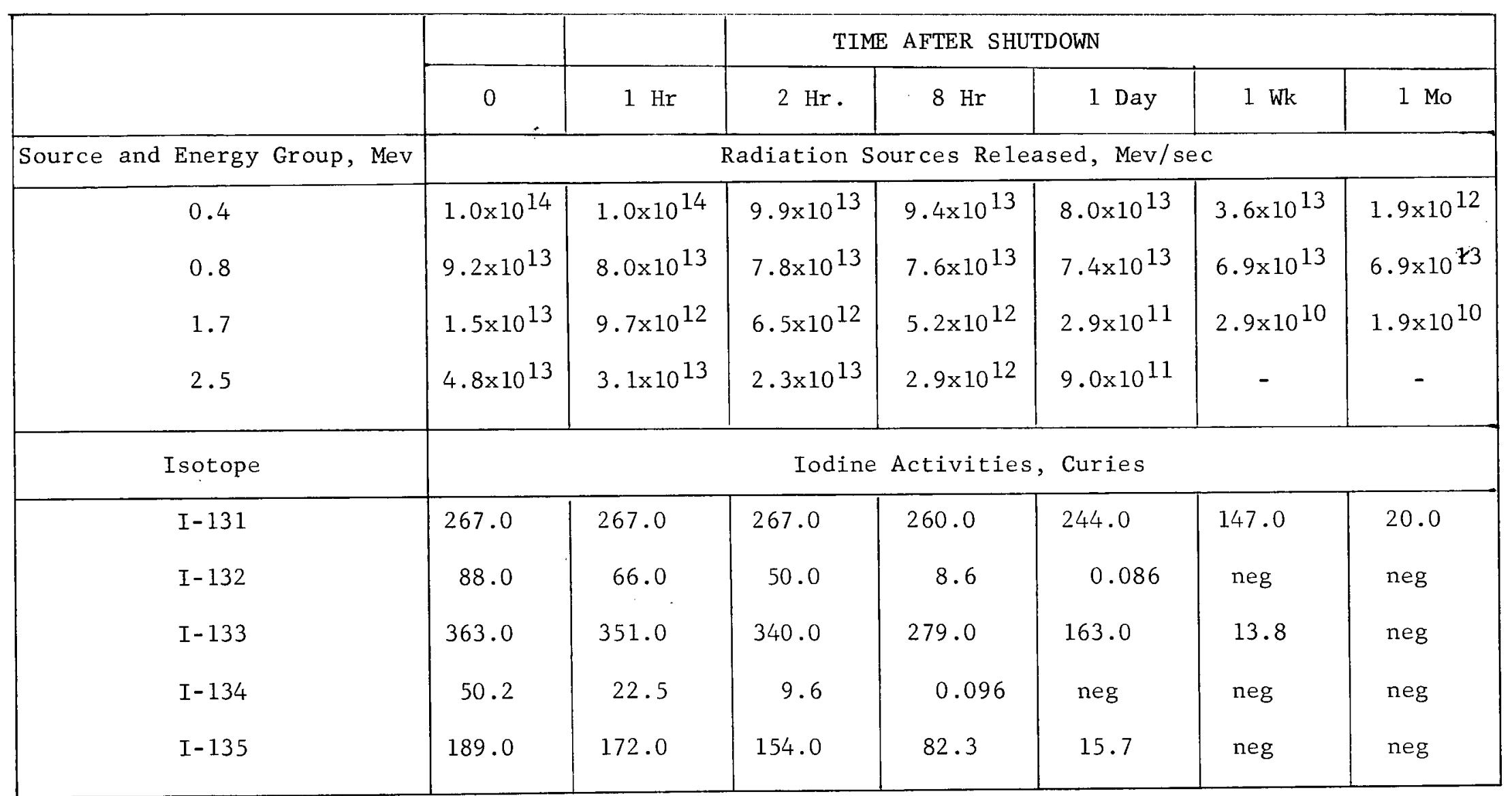


Table 13.5 Direct Gamma Sources Contained in Volume Control Tank for Reference One-Percent Fuel Defect Case

\begin{tabular}{|c|c|c|c|c|}
\hline \multirow[b]{2}{*}{$\begin{array}{l}\text { Energy } \\
\text { Group, Mev }\end{array}$} & \multicolumn{2}{|c|}{$\begin{array}{l}\text { SPECIFIC ACTIVITY } \\
\mathrm{Mev} / \mathrm{cc} \text { per sec }\end{array}$} & \multicolumn{2}{|c|}{$\begin{array}{c}\text { GROSS ACTIVITY } \\
\mathrm{Mev} / \mathrm{sec}\end{array}$} \\
\hline & $\begin{array}{l}\text { Liquid } \\
\text { Phase }\end{array}$ & $\begin{array}{l}\text { Vapor } \\
\text { Phase }\end{array}$ & $\begin{array}{l}\text { Liquid } \\
\text { Phase }\end{array}$ & $\begin{array}{l}\text { Vapor } \\
\text { Phase }\end{array}$ \\
\hline 0.4 & $7.5 \times 10^{5}$ & $8.3 \times 10^{5}$ & $9.0 \times 10^{11}$ & $9.9 \times 10^{11}$ \\
\hline 0.8 & $6.2 \times 10^{5}$ & $3.2 \times 10^{5}$ & $7.4 \times 10^{11}$ & $3.8 \times 10^{11}$ \\
\hline 1.7 & $10.5 \times 10^{4}$ & $2.5 \times 10^{5}$ & $12.5 \times 10^{10}$ & $3.0 \times 10^{11}$ \\
\hline 2.5 & $3.2 \times 10^{5}$ & $1.4 \times 10^{6}$ & $3.9 \times 10^{11}$ & $1.7 \times 10^{12}$ \\
\hline
\end{tabular}

\subsubsection{AUXILIARY SYSTEMS ACTIVITY (Continued)}

reactor coolant activity is a maximum, and that in the process all gaseous fission products are stripped, compressed, and stored in the gas decay tanks. The quantities of these gases can be obtained from Table 13.3. In so doing, the effect of decay during the shutdown and stripping operation is neglected. Table 13.61 ists separately the noble gas activities pertinent to the waste gas decay tank source, both as isotopic quantities and as photon energy group source strength.

\subsubsection{SPENT FUEL ACTIVITY}

The potential for release of fission product volatile activity contained in the pellet-cladding gap region of an irradiated fuel assembly is calculated from FIPCO-2 results for fuel which has undergone the maximum design burnup. It is assumed that a decay period of 90 hours elapsed in the process of shutting down the reactor, removing the head, and transferring the first fuel assembly to the spent fuel building.

For use in an evaluation of cladding damage in a spent fuel handing incident, the gap inventory of noble gas isotopes is 1isted in Table 13.7 on a per-megawatt basis. The potential release in the event a given number of fuel rods undergo clad ruptures would be obtained by multiplying the table value by the thermal power rating of the involved fuel rods. Non-gaseous isotopes are excluded, because of their essentially complete retention by the fuel and clad surfaces at the low temperatures of the spent fuel pit, and by the scrubbing effect of the fuel pit water should a release occur. 
Table 13.6 Direct Gamm Sources and Isotopic Inventory of Waste Gas Decay Tanks for a Reference One-Percent Fuel Defect Case

\begin{tabular}{|c|c|}
\hline $\begin{array}{l}\text { Energy } \\
\text { Group, Mev }\end{array}$ & $\begin{array}{c}\text { Gross Activity } \\
\text { Mev/sec }\end{array}$ \\
\hline 0.4 & $1.1 \times 10^{14}$ \\
0.8 & $5.0 \times 10^{12}$ \\
1.7 & $4.0 \times 10^{12}$ \\
2.5 & $2.8 \times 10^{13}$ \\
\hline Isotope & Inventory, Curies \\
\hline $\mathrm{Kr}-85 \mathrm{M}$ & 265 \\
$\mathrm{Kr}-85$ & 2556 \\
$\mathrm{Kr}-87$ & 149 \\
$\mathrm{Kr}-88$ & 428 \\
$\mathrm{Xe}-133$ & 32,200 \\
$\mathrm{Xe}-135$ & 897 \\
$\mathrm{Xe}-138$ & 65 \\
\hline
\end{tabular}


Table 13.7 Direct Gamma Sources and Isotopic Inventory for the Spent Fuel Gap Activity 90 Hours Following Shutdown

\begin{tabular}{|c|c|}
\hline $\begin{array}{c}\text { Energy Group, } \\
\text { Mev }\end{array}$ & $\begin{array}{c}\text { Noble Gas Activity (r/sec) } \\
(14 \text { Fuel Rods) }\end{array}$ \\
\hline 0.4 & $4.26 \times 10^{13}$ \\
0.8 & $8.0 \times 10^{7}$ \\
1.7 & Negligible \\
2.5 & Negligible \\
\hline Isotope & Inventory, Curies/Mwt \\
\hline Kr-85 & $1.8 \times 10^{2}$ \\
Kr-85m & $1.2 \times 10^{-4}$ \\
Kr-87 & $10^{-10}$ \\
Kr-88 & $10^{-10}$ \\
Xe-133m & 10.4 \\
Xe-133 & $1.5 \times 10^{3}$ \\
Xe-135 & $5.8 \times 10^{-3}$ \\
\hline
\end{tabular}




\section{SECTION 14 \\ HYPOTHETICAL ACCIDENT}

\subsection{HYPOTHETICAL ACCIDENT}

\section{1 .1 GENERAL}

To define an upper limit for accidental releases of radioactive material and consequent hazards to plant personnel or the general public an accident more severe than the credible accidents described in Section 10, Mechanical Malfunction Safety Analyses, is postulated. In considering this class of accidents, events were examined which might result from or be compounded by multiple failures. Simultaneous occurence, though, is not credible. Such accidents were evaluated on the basis of the quantity of radioactive material released to a region where direct radiation and escape to the environment would constitute a potential hazard.

Of the accidents considered, those which would result in the largest release in terms of potential environmental consequences are the class of major loss-of-coolant accidents with assumed partial melting of the reactor core. This conclusion follows from the supposition of the condition of maximum pressure in the containment and maximum availability of transportable radioactive material outside the core and reactor coolant system barriers. Reactivity accidents, proceeding to the point of destruction of the reactor vessel or its connecting piping, are not conceivable in the type of reactor proposed. This judgement is based on, (1) inherent limitations on reactivity insertion rates and, (2) the redundancy of the reactor protective systems.

However, to be conservative in postulating the hypothetical accident, a loss-of-coolant accident is considered. Such an accident resulting from complete circumferential severance of a main reactor coolant loop (27-1/2 inches ID) with unobstructed discharge from both ends of the severed pipe, compounded with the simultaneous failure of one of the two independent feedwater trains employed for safety injection, would result in radioactivity release which appears to represent a reasonably conceivable upper limit for this plant.

The type of compounded failure postulated is not considered credible in the light of design, construction, fabrication standards, and the following:

a. Experience indicates that reactor coolant loop piping materials (austenitic stainless steel) tend to fail in a ductile rather than a brittle manner. A brittle failure of large stainless steel piping, as far as is known, has never occurred in any steam electric power plant.

b. The safety injection system is normally actuated automatically by the coincident signals of low pressurizer pressure and level. In the unlikely event that the automatic actuation system failed, the safety injection system would be actuated by the operator. 
14.1.1 GENERAL (Continued)

c. Failure of a single channel of instrumentation to initiate a safety injection signal is provided by duplication of essential channels.

d. The possibility of the loss of a feedwater or safety injection pump, or of the valving required to bring both safety injection trains into effective action in the event of a loss-of-coolant accident, is remote.

\subsubsection{ANALYSIS}

An analysis of the postulated accident was performed using the methods covered in Paragraph 10.1, Loss of Coolant. To be conservative, the point of severance of the pipe was taken at the reactor inlet nozzle. In calculating the maximum extent of core damage resulting from the hypothesized break, credit was taken for the output of only one safety injection train in restoring coolant and in maintaining shutdown boron concentration.

Results showed the top of the core to be uncovered in six seconds and the bottom of the core in 7.3 seconds following such a break. Cooling of the exposed portion would occur by heat convection to the rising steam until the bottom of the core was uncovered. The essentially adiabatic heating of the pellets, cladding, and control rods would proceed until 64 seconds after the break, when the bottom of the core would be covered by safety injection water. Steam cooling would then resume until complete immersion of the core was affected.

The variation of cladding and pellet temperatures with time was determined by means of a digital code. The fraction of fuel rods with hot spot temperatures exceeding the clad-bursting temperature and the fraction of fuel pellets exceeding clad-melting temperatıre were each calculated as a function of time.

The fission product activity released into the sphere in such an accident would come from three principal sources:

a. The equilibrium activity of the reactor coolant at the time of the accident

b. The volatile activity present in the pellet-to-clad gap

c. The volatile and non-volatile fission products which diffuse from the $\mathrm{UO}_{2}$ fuel after the accident and are released through the failed cladding

The fission product activity released into the sphere for theoretical complete core meltdown is shown in Table 14.1. Quantities of the various radioactive elements were calculated from the data presented in Section 13, Fission Product Inventory, and represent end-of-life inventories from all 


\subsubsection{ANALYSIS (Continued)}

significant contributors. The diffusion of fission products from $\mathrm{UO}_{2}$ after the accident was estimated conservatively by assuming that 100 percent of the noble gases, 50 percent of the halogens, and one percent of the nonvolatile fission products were instantly released from the core. Of the 50 percent, an additional 50 percent reduction due to halogen plate-out on the containment structure was assumed. Thus, only 25 percent of the halogens would be available for release to the environment.

Table 14.1 Theoretical Complete Core Meltdown Fission Product Sources

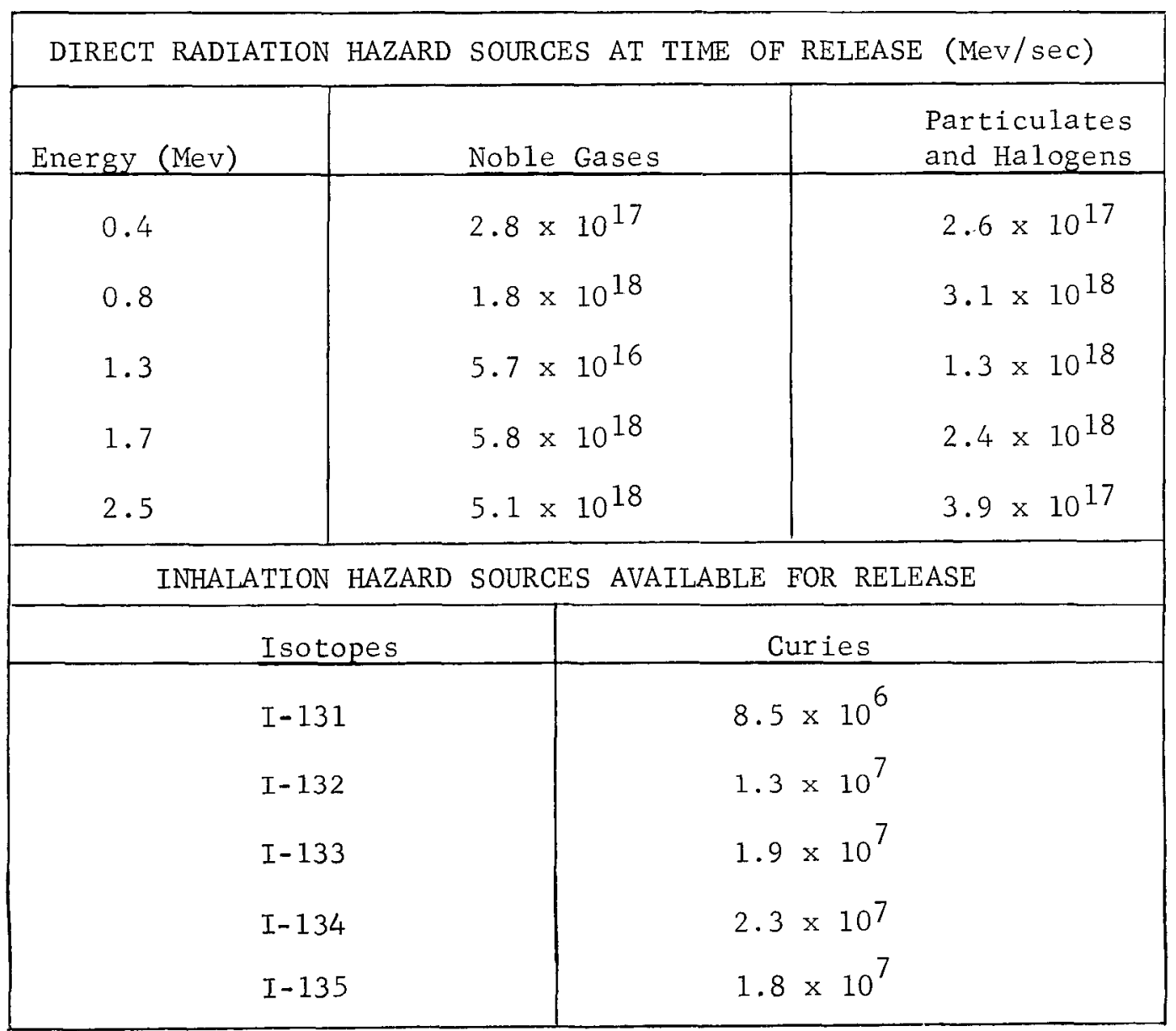

The analysis described in Paragraph 10.1, Loss of Coolant, shows that an amount equivalent to approximately 25 percent (25 percent of Table 14.1 sources) derived from complete core meltdown, results from a core consisting of zirconium alloy clad fuel rods, when the effect of a single safety injection train is included.

Environmental consequences of the hypothetical accident are evaluated in Section 15, Environmental Consequences. 


\section{SECTION 15 \\ ENVIRONMENTAL CONSEQUENCES}

\subsection{ENVIRONMENTAL CONSEQUENCES OF HYPOTHETICAL ACCIDENT}

\subsubsection{GENERAL}

In considering the environmental consequences of the hypothetical accident described in Section 14, calculations were made to determine integrated whole body, thyroid, and bone doses as a function of distance from the containment vesse1. Integrated whole body doses due to direct radiation exposure from the containment vesse 1 as well as due to immersion in a radioactive cloud formed by leakage of fission products from the containment vessel were determined. Integrated thyroid and bone doses were calculated to determine the effects of inhalation of certain fission products leaking from the containment vessel.

\subsubsection{BASIS FOR DOSE CALCULATIONS}

The expected environmental conditions, related parameters, and assumptions made in performing the dose calculations are discussed below. Those factors that would serve to reduce the calculated doses have been intentionally neglected in order to present the results in the most conservative manner.

\section{Factors Affecting Radioactive Inventory in Containment Sphere}

As stated in Section 14, the quantities of radioactive isotopes in the containment vessel can be represented as a percentage of the core fission product inventories from all significant contributors for the longest fue 1 cycle at full power operation ( $1,347 \mathrm{Mwt}$ ).

Sources used for calculating doses outside of the containment vessel are 25 percent of values listed in Table 14.1, for cores with zirconium alloy fuel cladding.

Although there is a significant delay from the time the activity release of radioactive fission products from the overheated fuel is mixed with the containment vessel atmosphere, instantaneous mixing in the containment is assumed. Radioactive decay during residence in the containment is taken into consideration in all source data.

The containment vessel air recirculation system has been designed to continue operation through any loss of coolant accident. Operation of this system will reduce dispersed particulate and halogen fission product concentrations in the containment vessel atmosphere. However, credit was not taken for this system in the calculations for determining environmental consequences.

The containment vessel spray system will substantially lower the halogen activity dispersed to the containment vessel atmosphere, although credit for this factor was not taken in determining environmental consequences. 


\subsubsection{BASIS FOR DOSE CALCULATIONS (Continued)}

Factors Affecting Dose from Direct Radiation from The Containment $\underline{\text { Vessel }}$

In calculating doses due to radioactive isotopes retained in the containment vesse1, attenuation of radiation by the steel containment vessel shel1, internal concrete shields and structures, and air are taken into account. Terrain around the plant will reduce the dose by a significant amount, depending on the specific offsite locations. However, no credit has been taken for the shielding affect of topography.

Factors Affecting Doses Due to Radioactivity Leaking from Containment Vesse1

The design and construction of the Containment Sphere was based upon a specified constant leakage rate of 0.1 percent of the Containment sphere vessel volume per 24 hours at design pressure. However, for the purpose of arriving at a containment leakage rate under assumed hypothetical accident conditions, a parametric study was performed for leakage rates of 0.5 percent and 1.9 percent of the containment volume per 24 hour period.

The foregoing assumptions are conservative, in that the pressure in the containment vesse1 will vary from the peak pressure following an accident to lower pressures as the containment vessel atmosphere is cooled. The initial leak rate tests performed in May of 1965 indicate that the leakage at design pressure was 0.02 percent volume per 24 hours.

Atmospheric dilution of radioactive concentrations, leaking from the containment vessel subsequent to the postulated accident, has been estimated by using methods and data outlined by F. A. Gifford. 1 For the zero-to-two hour dose calculations pertinent to the exclusion area, slightly stable atmospheric conditions (Gifford's Condition E) and a wind speed of four mph are assumed. Data presented in Paragraph 2.2, shows that winds with velocities greater than four mph occur 87 percent of the time on an annual basis. Data collected at and adjacent to the plant site for six months shows that winds speed in excess of four mph 81 percent of the time. For long term (zero-to-infinity) dose calculations, average meteorological conditions in the vicinity of the plant, based on data presented in Paragraph 2.2, are assumed to prevail. These correspond to slightly unstable atmosphere (Gifford's Condition $C$ ) and a wind speed of eight mph.

It is assumed that fission product leakage from the containment vessel takes place essentially at ground level and that the motion of the air flowing past the containment disperses the fission products rapidly into a volume equal to the product of the projected vertical area of the containment vessel and the wind speed.2 As the resultant cloud moves downwind, fission products are further diluted by the mechanisms of diffusion and air turbulence.

Calculations of doses resulting from containment vessel leakage are conservatively based on the assumption that the wind blows from the same direction during the entire course of the hypothetical accident, and that the assumed atmospheric conditions persist for the entire period. Atmospheric dilution 


\subsubsection{BASIS FOR DOSE CALCULATIONS (Continued)}

multipliers, as a function of distance, are shown in Figure 15.1.

Calculation of whole body gamma dose from the cloud is based on immersion

in a semi-infinite medium.

Thyroid doses are calculated using the methods outlined in TID-14844.3

No credit has been taken for depletion or reduction of cloud activity due to radioactive decay during transport from the containment vessel to the receptor.

\subsubsection{DOSES DUE TO ACTIVITY RETAINED IN CONTAINMENT}

The integrated direct radiation whole body doses for 25 percent equivalent core meltdown are shown in Figure 15.2. A dose of 25 rem occurs at a distance of 1,510 feet from the containment vessel centerline for the zero-totwo hour case, and 2,510 feet for the zero-to-infinity case.

\subsubsection{DOSES DUE TO ACTIVITY LEAKING FROM THE CONTAINMENT}

\section{Inhalation Doses}

Integrated thyroid doses resulting from inhalation of radioiodine released from the containment vessel are shown in Figures 15.3 and 15.4.

Figure 15.3 shows the zero-to-two hour and zero-to-infinity thyroid doses for the 25 percent meltdown accident and 0.5 percent per day containment leak rate. For the zero-to-infinity doses, 300 rem was calculated to occur 3,600 feet from the containment. The thyroid doses are less than 300 rem at all distances beyond the site boundary for the zero-to-two hour case. Figure 15.4 shows thyroid doses for the 25 percent meltdown accident and 1.9 percent per day containment leak rate. Doses for the zero-to-two hour case are less than 300 rems beyond one-half mile, and are less than 300 rems beyond 8,200 feet from the containment vessel for the zero-to-infinity case.

Consideration has been given to the bone dose which could result from inhalation of the cloud downwind from the sphere following the hypothetical accident. Calculations show that the integrated bone dose during the first two hours for the 25 percent meltdown case 1.9 percent containment leak rate is less than 25 rem at 2,250 feet. The following factors significantly reduce the resulting offsite doses over long periods of time for strontium 90 confined in the containment:

a) Operation of the containment vesse1 spray system and containment vessel air recirculation system will effectively remove strontium from the containment vessel atmosphere; moreover, neither immediate operation nor high efficiency of these systems is necessary to limit bone doses to small values. 


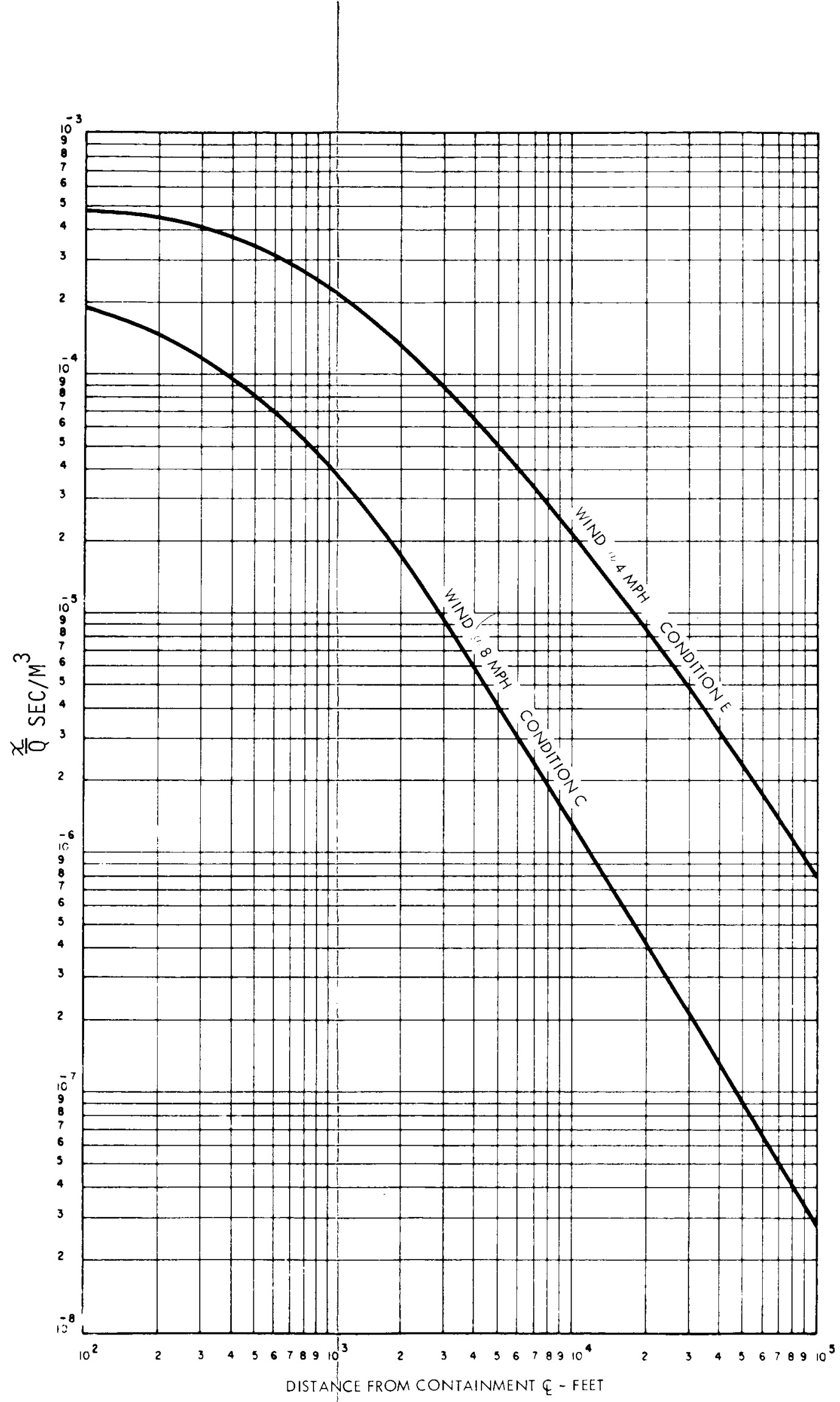

FIGURE 15.1 DILUTION MULTIPLIÉRS 


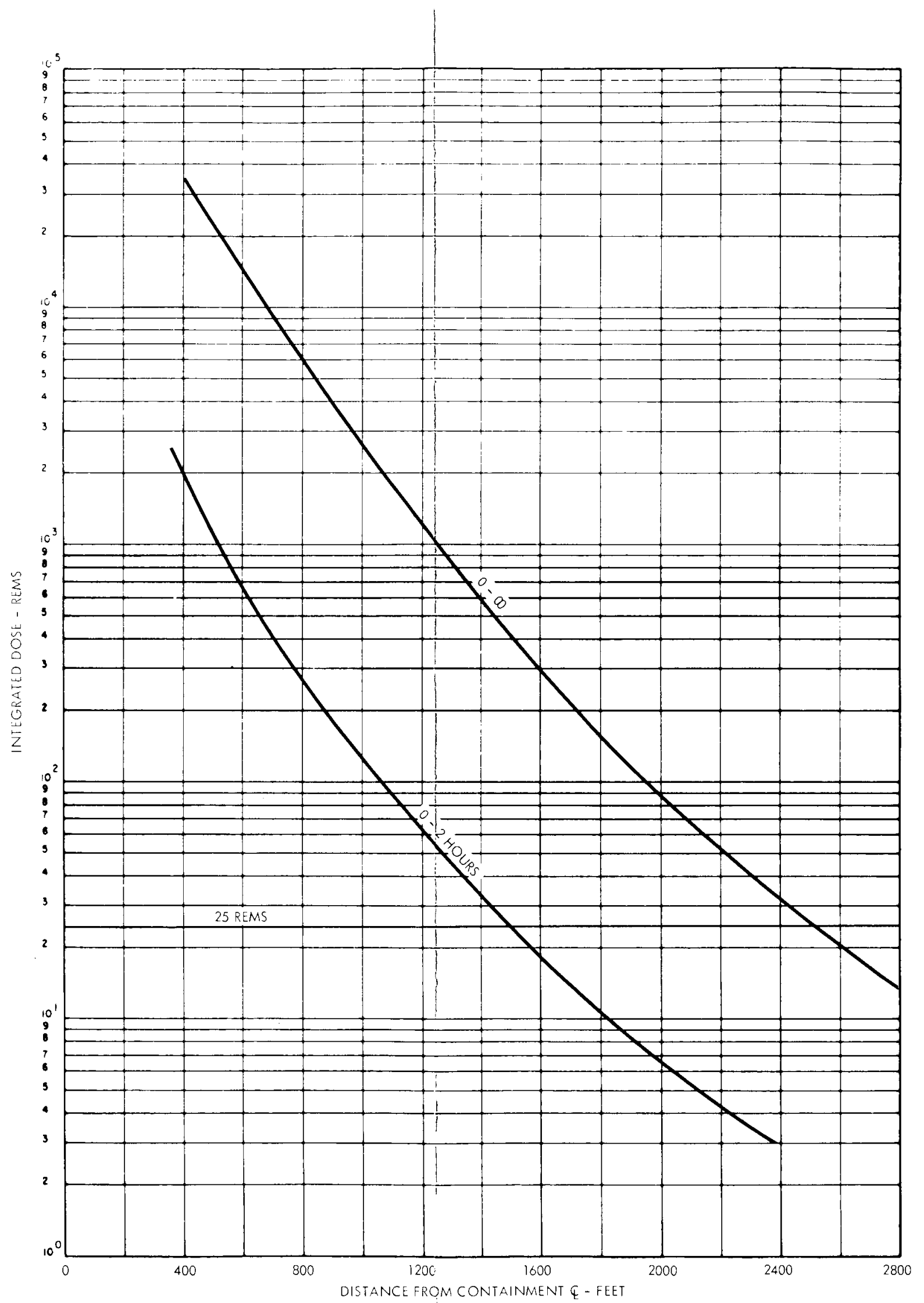

FIGURE 15.2 DIRECT DOSE, 25 PERCENT CORE MELTDOWN 


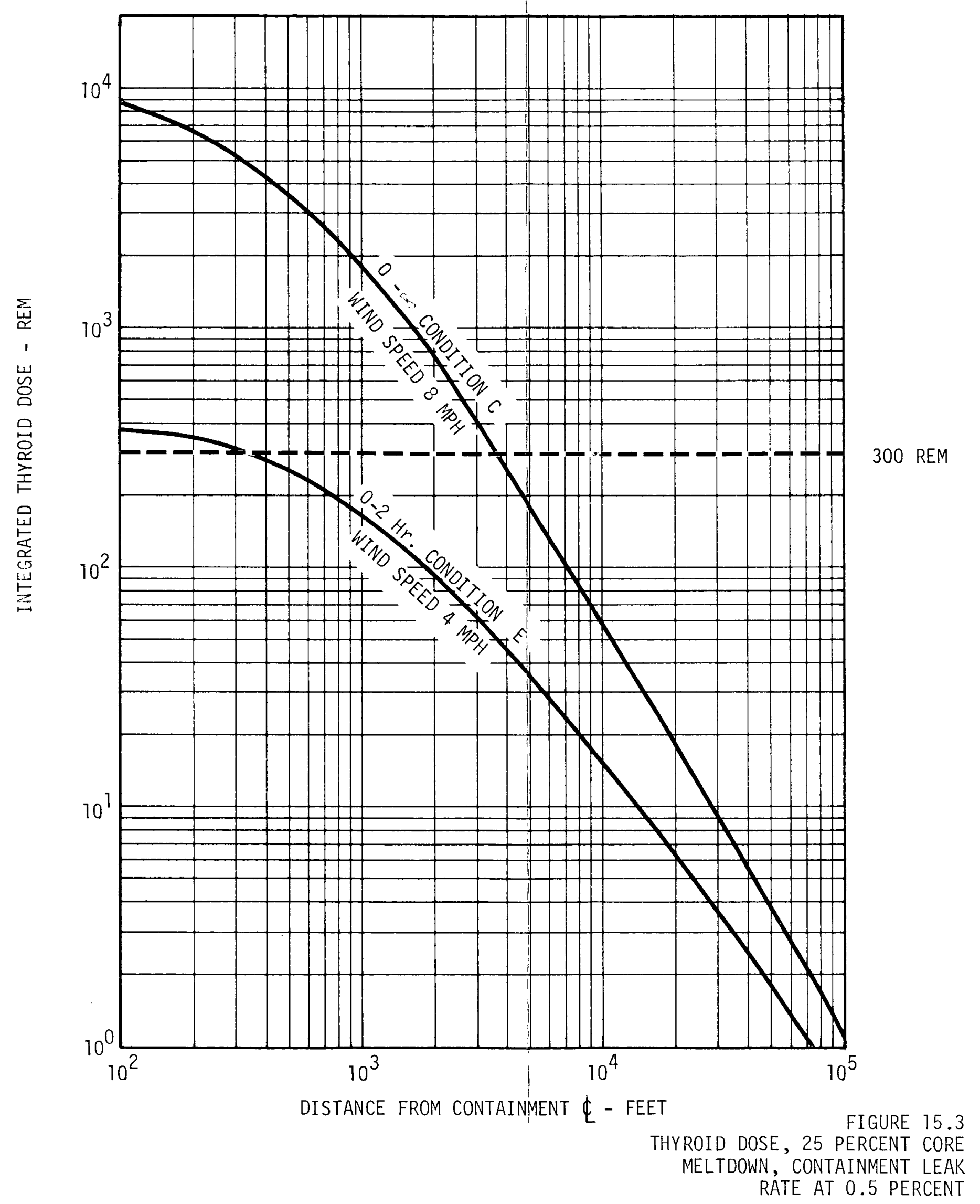




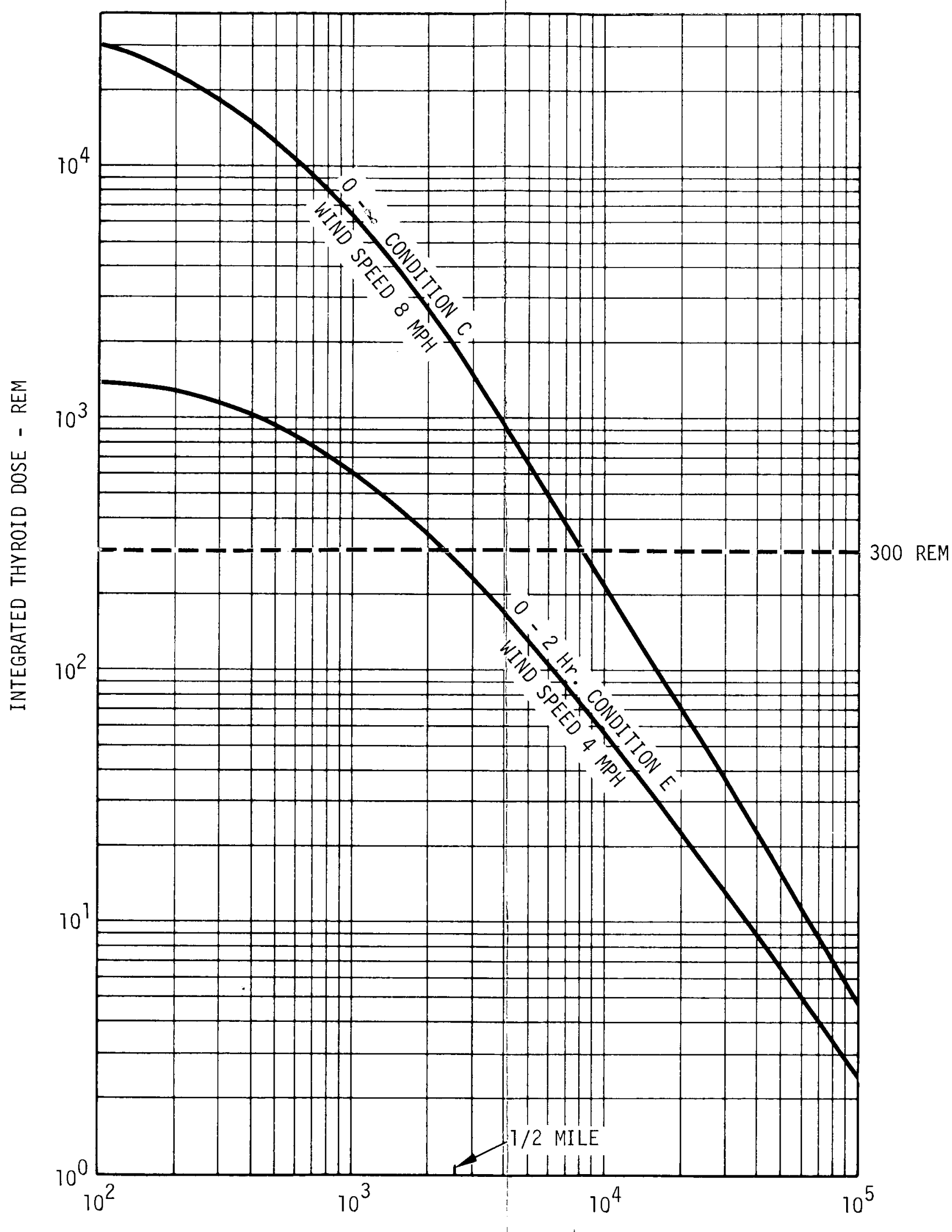

DISTANCE FROM CONTAINMENT \& - FEET

FIGURE 15.4

THYROID DOSE, 25 PERCENT COFE MELLTDOWN, CONTAINMENT LEAK RATE AT 1.9 PERCENT PER DAY 
15.1.4 DOSES DUE TO ACTIVITY LEAKING FROM THE CONTAINMENT (Continued)

b) Decrease in containment vessel pressure with time will decrease the containment vessel leakage below the assumed leakage rates, further decreasing offsite bone doses.

c) Average meteorological conditions and wind diversification will significantly lower long-term bone doses.

Whole-Body Immersion Doses

Direct radiation doses due to whole-body immersion in the containment leakage cloud are shown in Figures 15.5 and 15.6.

\section{$\underline{\text { Rain-Out Analysis }}$}

Calculations were made to determine the consequences of rain-out depletion of the containment vessel leakage cloud and the resultant ground deposition at off site locations.

Rainfall and wind data for Laguna Beach, Oceanside, and a location adjacent to the plant site have been reviewed; they show good correlation with the general storm patterns in Southern California and are believed to represent the rainfall pattern at the site.

Comparing wind data with corresponding periods of rainfall data, precipitation and wind conditions were established. The rainfall analysis is based upon the following meteorological data pertinent to the site and San Clemente:

a) From the 27-month rain data in this area, wind movement at the site is toward the San Clemente sector (ESE, SE, SSE winds) 24 percent of the time during rain conditions.

b) However, the most probable case of annual wind distribution is that 5 percent of the time, ESE, SE, and SSE winds prevail (Reference Paragraph 2.2.6).

c) Maximum rainfall duration is 10 hours.

d) Average rainfall duration is four hours.

e) Maximum hourly rainfall is 0.33 inch.

f) Average hourly rainfall is 0.07 inch.

Predominant rainfall conditions are caused by an extensive low pressure area with counter-clockwise air movement toward the coast line. Therefore, the probable winds from the ESE, SE and SSE are analyzed.

In the analysis of results for rain-out activity released following the hypothetical accident at 1.9 percent per day containment leak rate, the following two principal guidelines were used: 


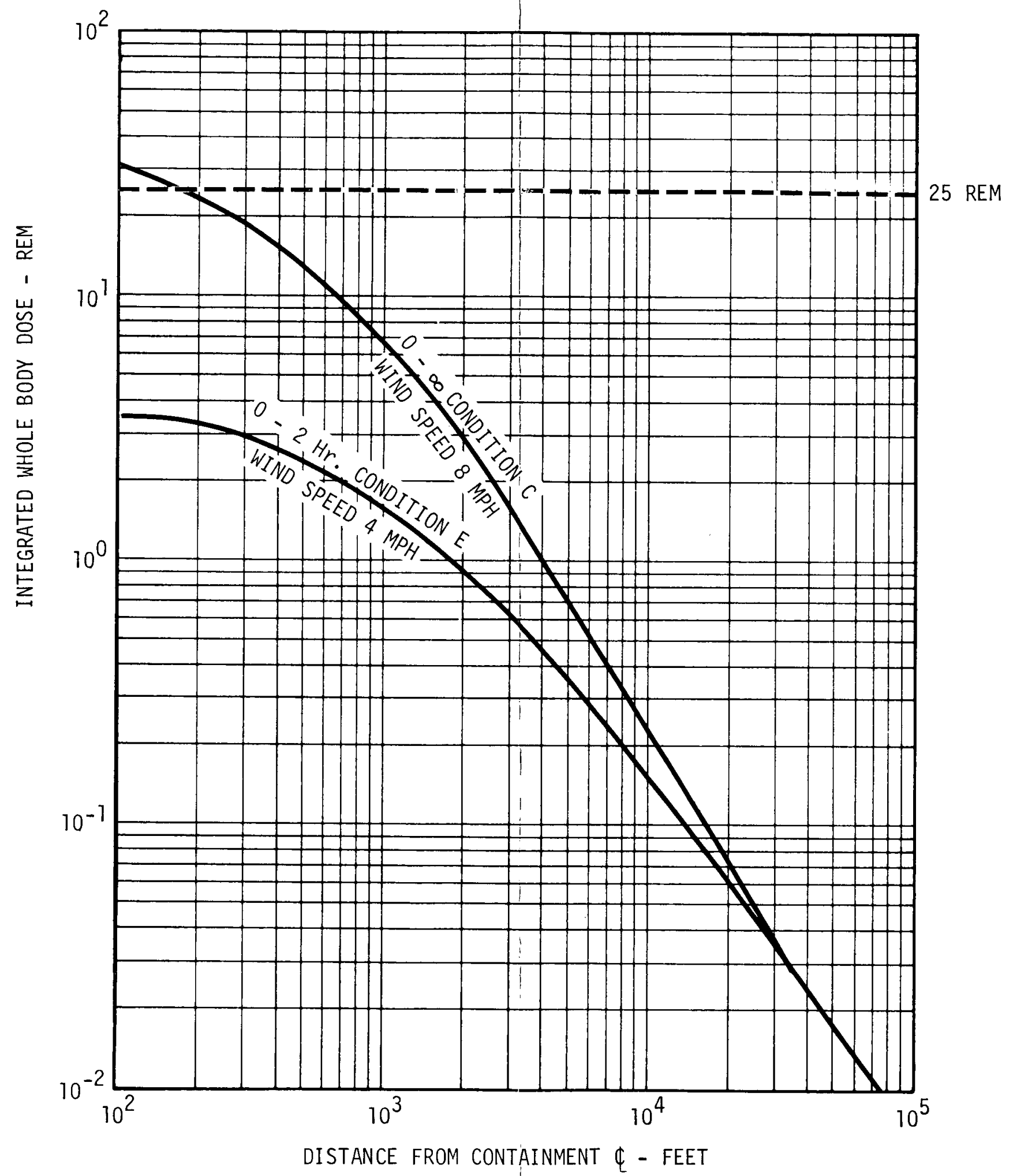

FIGURE 15.5 WHOLE BODY IMMERSION DOSE, 25 PERCENT CORE MELTDOWN, CONTAINMENT LEAK RATE AT 0.5 PERCENT PER DAY 


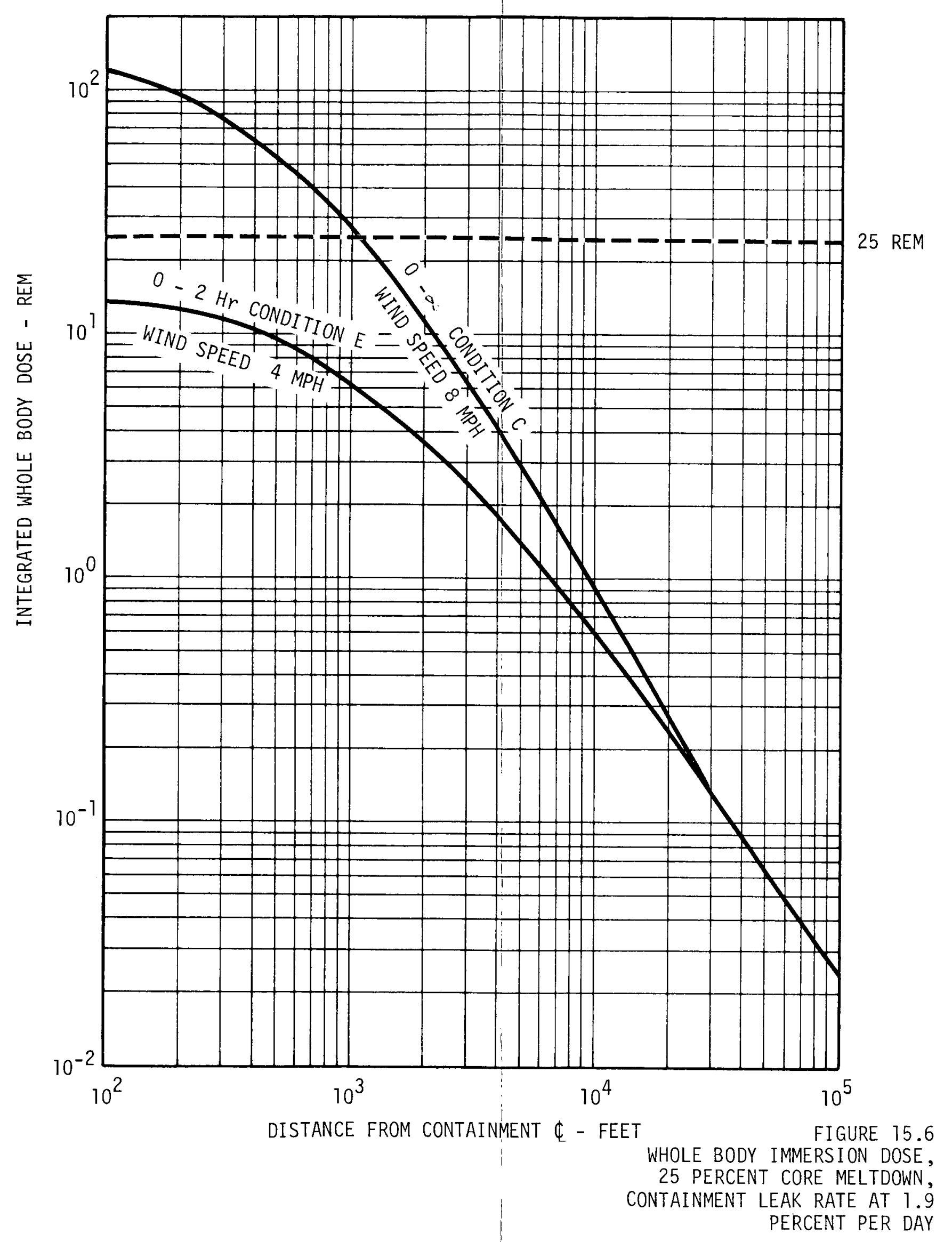




\subsubsection{DOSES DUE TO ACTIVITY LEAKING FROM THE CONTAINMENT (Continued)}

a. A simple Gaussian interpolation of Sutton's dispersion model is applicable. 4

b. Based upon the above, meteorological parameters assumed for the calculations are:

- The maximum single rain duration with winds from SE, ESE, and SSE directions is 10 hours.

- The frequency of wind toward San Clemente from the site for rainfall condition is 24 percent (Reference is Amendment $\#^{4}$ PHSR Section 非3).

- The wind speed during rainfal1 is $10 \mathrm{mph}$.

- The source term used for this analysis is the inventory for release from the hypothetical accident, 25 percent meltdown case.

- The probability of particles being removed by rainfall is taken to be $7 \times 10^{-4}$ per second. 5

The calculated resultant deposition of radioactivity five miles downwind from the plant site is:

$$
\begin{aligned}
& \text { I }^{131} 1.15 \times 10^{-5} \text { curies per square meter } \\
& \text { Sr90 } 6.1 \times 10^{-8} \text { curies per square meter }
\end{aligned}
$$

Using an hourly rainfall of 0.15 inch per hour, and a frequency of ESE-SESSE winds of 24 percent, the following unlikely but interesting comparison $c$ an be determined:

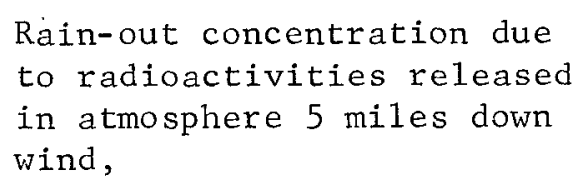

$$
\begin{aligned}
& I^{131} 8.75 \times 10^{-8} \mu \mathrm{c} / \mathrm{cc} \\
& \operatorname{Sr} 904.68 \times 10^{-10} \mu \mathrm{c} / \mathrm{cc}
\end{aligned}
$$

MPC (10 CER 20)

$3 \times 10^{-7} \mu \mathrm{c} / \mathrm{cc}$

$3 \times 10^{-7} \mu \mathrm{c} / \mathrm{cc}$

However, more realistically, the washout will not be collected in a container but will be distributed on the ground as any other rainfall. Consequently, further dilution factors can be realized from such items as additional rain during any one rainfall period, when the winds are not from the site.

It is therefore concluded that, for areas downwind of the plant site, continuous rainfall conditions will not constitute a potential danger from rain-out of released radioactivity. 


\subsubsection{DISCUSSION OF RESULTS}

An evaluation of the foregoing calculation results was made to compare the calculated separation distances with available physical separation distances, as defined by AEC Guidelines set forth in $10 \mathrm{CFR}$ 100, Section 100.3, Definitions. The calculated results are compared with available distances in Table 15.1.

A comparison of distances shown in Table 15.1 reveals that thyroid doses govern determination of the exclusion area, low population zone, and population center distance.

It $c$ an be seen that sufficient physical separation distances are available to ensure that doses of 300 rems to the thyroid and 25 rems to the whole body will not be exceeded. As a result, the environmental consequences of the hypothetical accident are not considered to represent an undue hazard to public health and safety.

\subsubsection{REFERENCES}

1. Gifford, F. A. "Use of Routine Meteorological Observations for Estimating Atmospheric Dispersion." Nuclear Safety (June 1961)

2. Gifford, F. A. "Atmospheric Dispersion Calculations Using the Generalized Gaussian Plume Mode1." Nuclear Safety (December 1960)

3. "Calculation of Distance Factors for Power and Test Reactor Sites." TID-14844 U.S. Atomic Energy Commission, Division of Technical Information, (March 1952)

4. O. G. Sutton, A Theory of Eddy Diffusion in the Atmosphere Proc. Roy Soc. (London) 135A: 1431932

5. A. C. Chamberlain, Aspect of Travel and Deposition of Aerosol and Vapor Clouds, British Report AERE-HP/R-1261 Sept. 17, 1953 
Table 15.1 Distance Summary Table

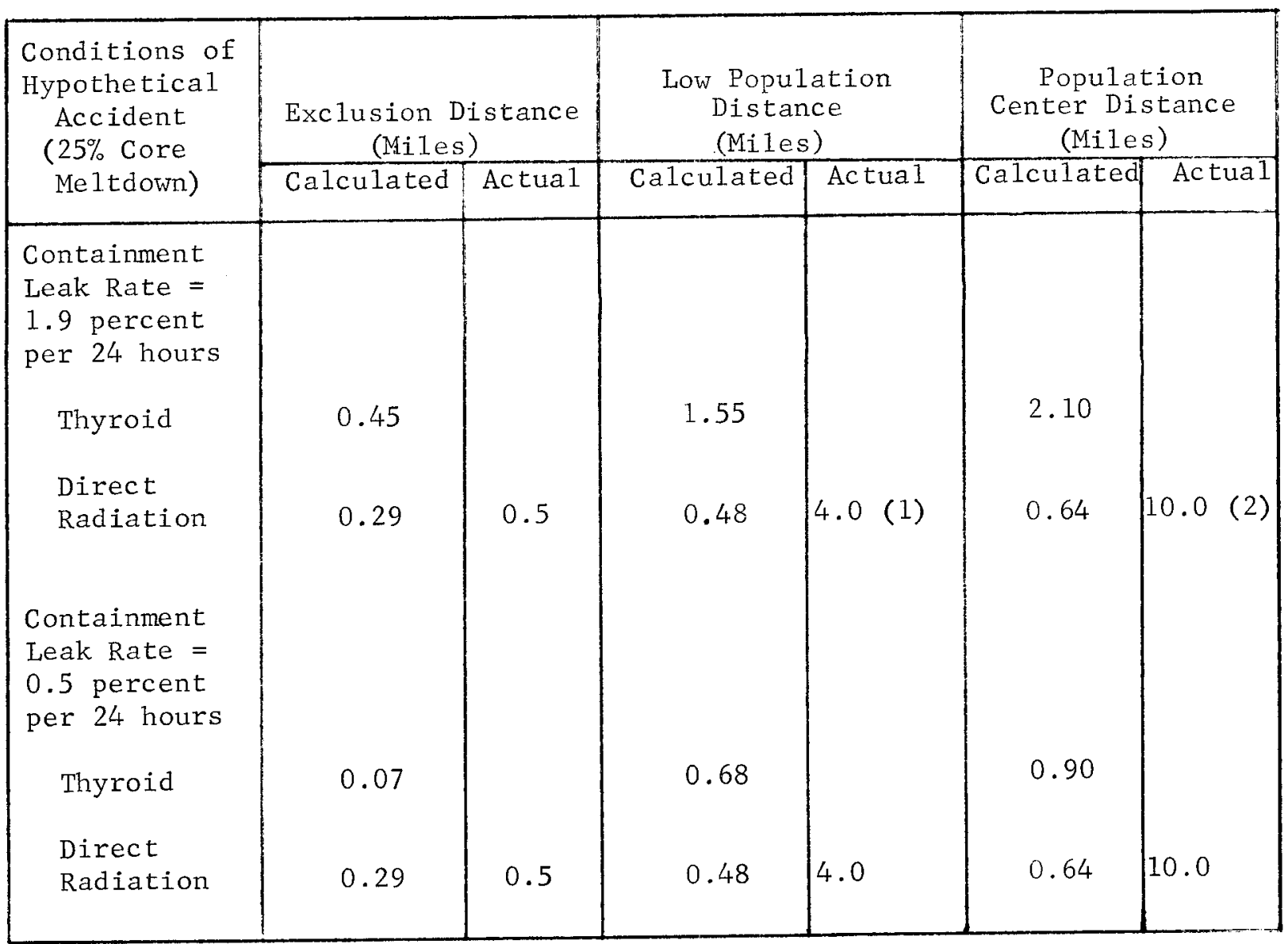

(1) 4.0 miles is the distance to the nearest city - San Clemente.

(2) Total estimated population within the 10 -mile radius is 25,000 . 


\subsection{ENVIRONMENTAL CONSEQUENCES OF OTHER MALFUNCTIONS}

\subsubsection{LOSS OF COOLANT ACCIDENT}

Integrated whole-body and thyroid doses were calculated for the loss of coolant accident described in Paragraph 10.1. Parameters similar to those used for the hypothetical accident described in Section 14 were used in the calculations. Radioactivity released to the containment is assumed to be that contained in the primary coolant, except that 50 percent of the radioactive iodine is assumed to be plated out on reactor structures and within the secondary shield.

Results of calculations for direct radiation whole-body doses are shown in Figure 15.7. These cover both zero-to-two-hour and zero-to-infinity exposure periods. In both cases, calculated doses beyond the plant boundary, are well below the reference values set forth in 10 CFR 100 .

The results of thyroid dose calculations, shown in Figures 15.8 and 15.9, cover zero-to-two-hour and zero-to-infinity cases. Thyroid doses are well below 300 rem at all distances from the containment.

The whole-body immersion doses were found to be correspondingly low. Like the direct radiation and thyroid doses, they do not represent an undue hazard to public health and safety in the unlikely event of an accidental release of the type postulated.

\subsubsection{WASTE GAS ACCIDENT}

Postulated conditions that would result in the release of radioactive gases from waste gas storage tanks are described in Paragraph 10.7. Environmental consequences of the postulated release have been calculated, based on the following parameters:

a. The cubicle in which release takes place is continuously ventilated at the rate of $470 \mathrm{cfm}$.

b. Activity from the waste gas cubicle is mixed with other ventilation air in the 100-foot ventilation stack, discharging air at the rate of $40,000 \mathrm{cfm}$ and at a velocity of $42 \mathrm{fps}$.

c. Meteorological conditions were taken as slightly stable atmosphere (Gifford's Condition E), with a wind speed of four mph for the entire course of the accident

d. Exposure to radiation would be from two sources: gamma radiation from the plume passing over the receptor, and gamma and beta radiation due to whole-body immersion

e. A total of 20,000 curies would be released during the course of the accident $(10,000$ curies from each of two tanks). 


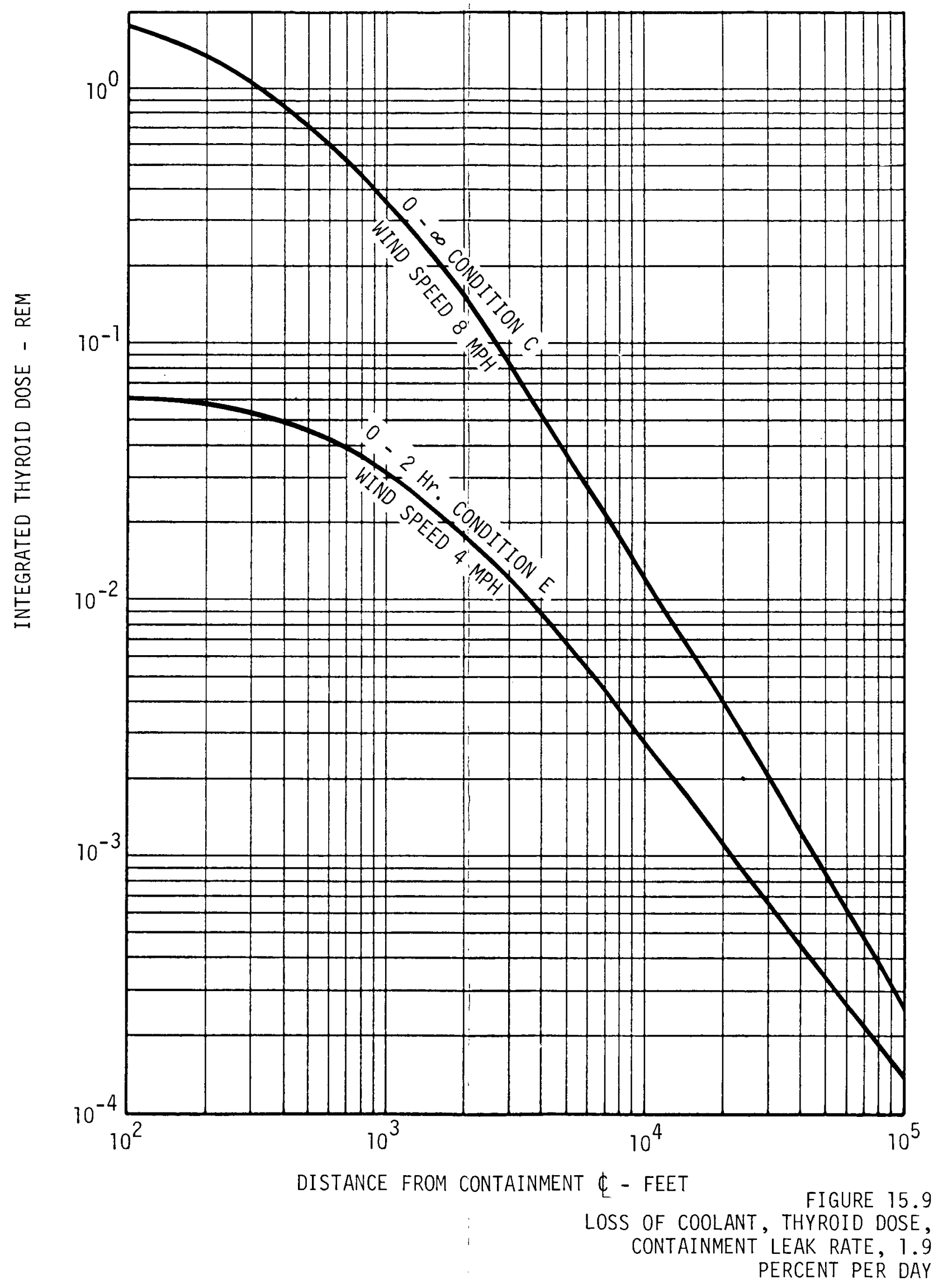




\subsubsection{WASTE GAS ACCIDENT (Continued)}

Dilution multipliers shown in Figures 15.10 and 15.11 were calculated using an average effective stack height derived by averaging results obtained from Bosanquet and Braynt Davidson. Figure 15.10 shows dilution multipliers for plume centerline concentrations as a function of distance, while Figure 15.11 shows ground concentration dilution multipliers for the same stack release conditions.

Whole body doses due to gamma exposure were calculated using average plume concentrations, and a cylindrical source geometry model. The results of the calculations appearing in Figure 15.12 represent the total integrated dose received along the clouc axis for the duration of the accident. At all distances greater than 100 feet from the stack centerline, the doses are less than $0.01 \mathrm{rem}$.

\subsubsection{STEAM-GENERATOR TUBE RUPTURE}

Environmental consequences of a steam-generator tube rupture accident, described in Paragraph 10.5 were analyzed using the following parameters:

\section{Initial Discharge of Coolant Activity to Atmosphere}

a. Release of activity will be through any one of four steam dump valve risers

b. Duration of atmospheric discharge will be 60 seconds

c. Total activity release through the riser will be:

$$
\begin{aligned}
& \text { equivalent } I-13 I=0.005 \text { curies } \\
& \text { equivalent } X e-133=850 \text { curies }
\end{aligned}
$$

d. There will be slightly stable atmospheric conditions with a wind speed of four mph

e. There will be continuous point source discharge from the steam dump riser (elevation 63 feet)

\section{Discharge of Coolant Activity From Plant Stack}

a. Discharge duration will be 4.3 hours

b. Total release will amount: to:

equivalent $I-131=0.031$ curies

equivalent $\mathrm{Xe}-133=26,443$ curies

c. Atmospheric conditions will remain slightly stable with a wind speed of four mph for entire course of the accident. 


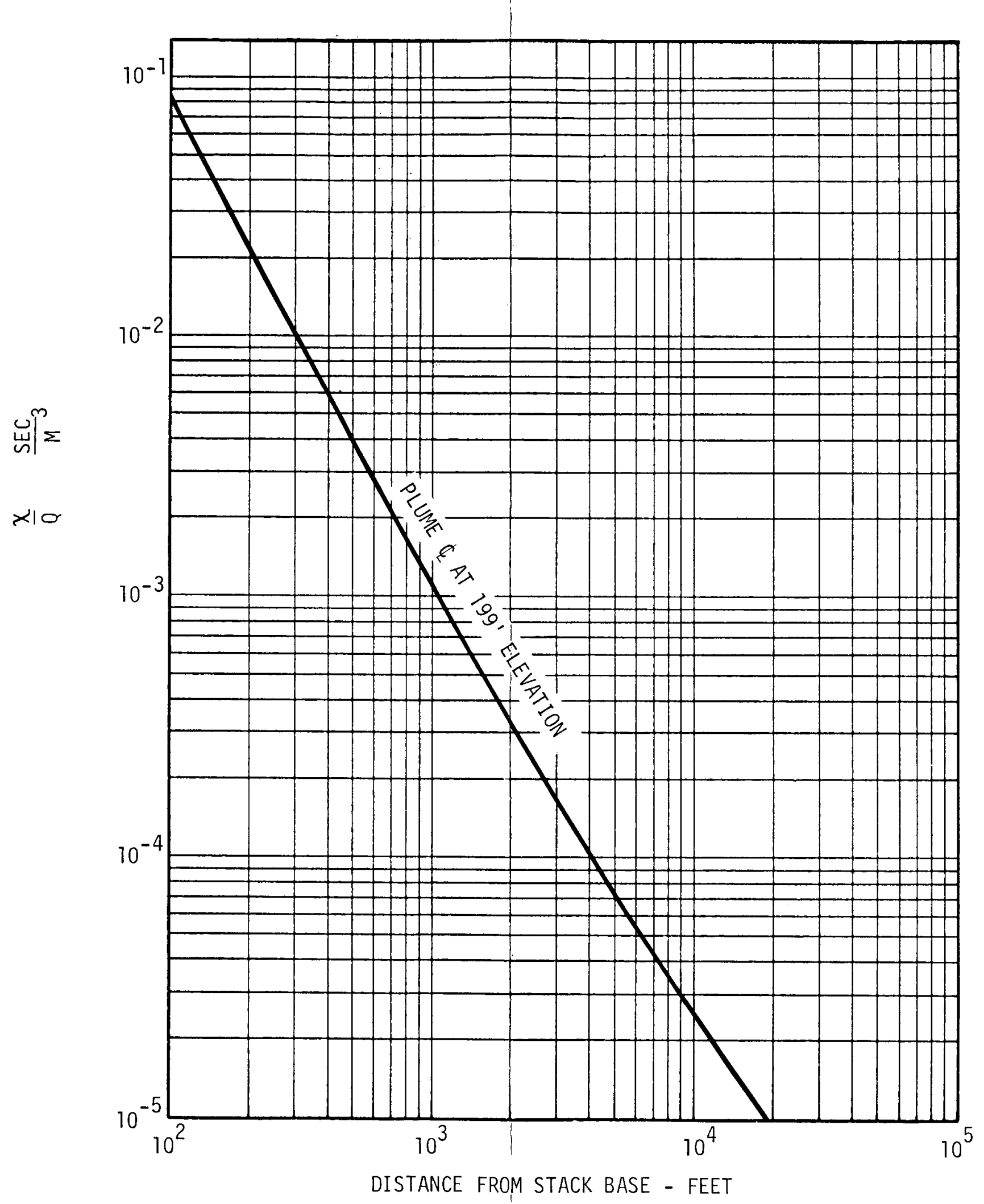

FIGURE 15.10

DILUTION MULTIPLIER, SLIGHTLY

STABLE ATMOSPHERE, WIND SPEED

FOUR MPH 


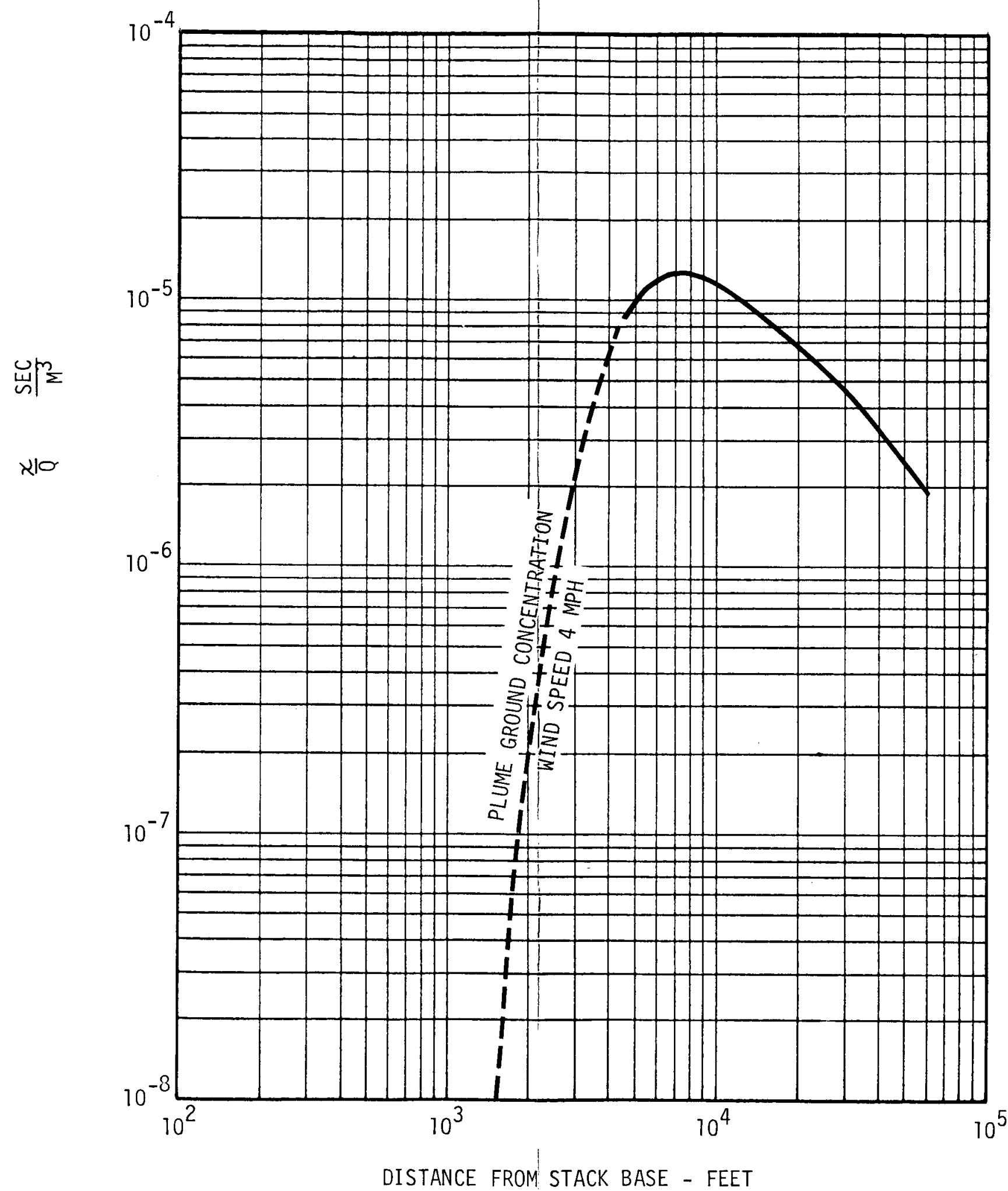

FIGURE 15.11

DILUTION MULTIPLIER, SLIGHTLY

STABLE ATMOSPHERE (STACK DISCHARGE VELOCITY, 42 FPS; STACK HEIGHT, 100 FEET) 


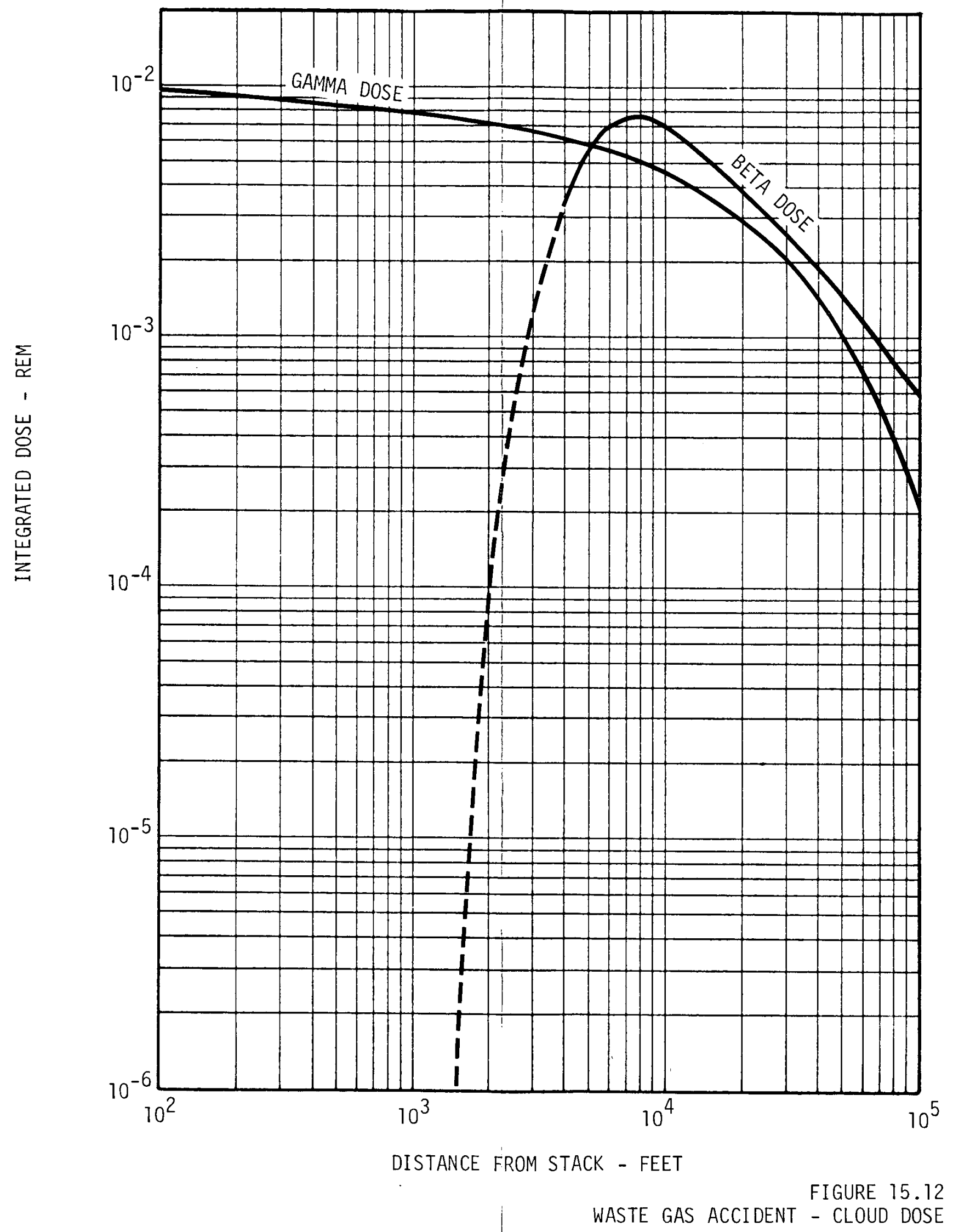




\subsubsection{STEAM GENERATOR TUBE RUPTURE (Continued) \\ Atmospheric Dilution Multipliers}

The calculation model for atmospheric dilution multipliers is the same as that used for the waste gas accident evaluation.

\section{Results}

Results of the analysis show that integrated doses to the whole body and thyroid are wel1 below reference values, and thus do not represent a hazard to public health and safety. Effects of postulated discharge from the steam dump risers can be considered negligible.

Figure 15.13 shows the calculated whole body dose resulting from plant stack discharge for exposures of $0-2$ and $0-4.3$ hours, respectively.

\subsubsection{FUEL HANDLING ACCIDENT}

For the purpose of evaluating assumed environmental consequences that might result from the damaging of an entire row of fuel rods (14 fuel rods) and resultant breaching of the cladding, two cases were considered.

In the first case it was assumed that rod damage would occur during fuel handling in the refueling pit. It was further assumed that the entire noble gas inventory from the 14 rods mixed with the containment air, and was subsequently discharged through the plant ventilation stack. Air flow from the containment at this time was assumed to be $20,000 \mathrm{cfm}$.

In the second case, rod damage was assumed to have taken place in the spent fuel storage pit, with the resulting noble gas release being discharged into the spent fuel building prior to being exhausted to the atmosphere through the plant ventilation stack.

The resulting whole body doses were calculated, using the model developed for the waste gas accident, together with source data provided in Paragraph 10.3 .

As may be seen from the curves presented in Figure 15.14, the integrated whole body doses are very sma11.

\subsubsection{ROD EJECTION ACCIDENT}

The postulated release of radioactivity into the containment sphere as a result of the rod ejection accident described in Paragraph 9.6 was assumed to be equivalent to that released as a result of one percent of the core melting. The associated rate of energy release, due to coolant blow-down, is of the order of a four inch pipe break.

Assumptions and parameters used to determine whole-body and thyroid doses for the hypothetical and loss of coolant accident are assumed to be the 


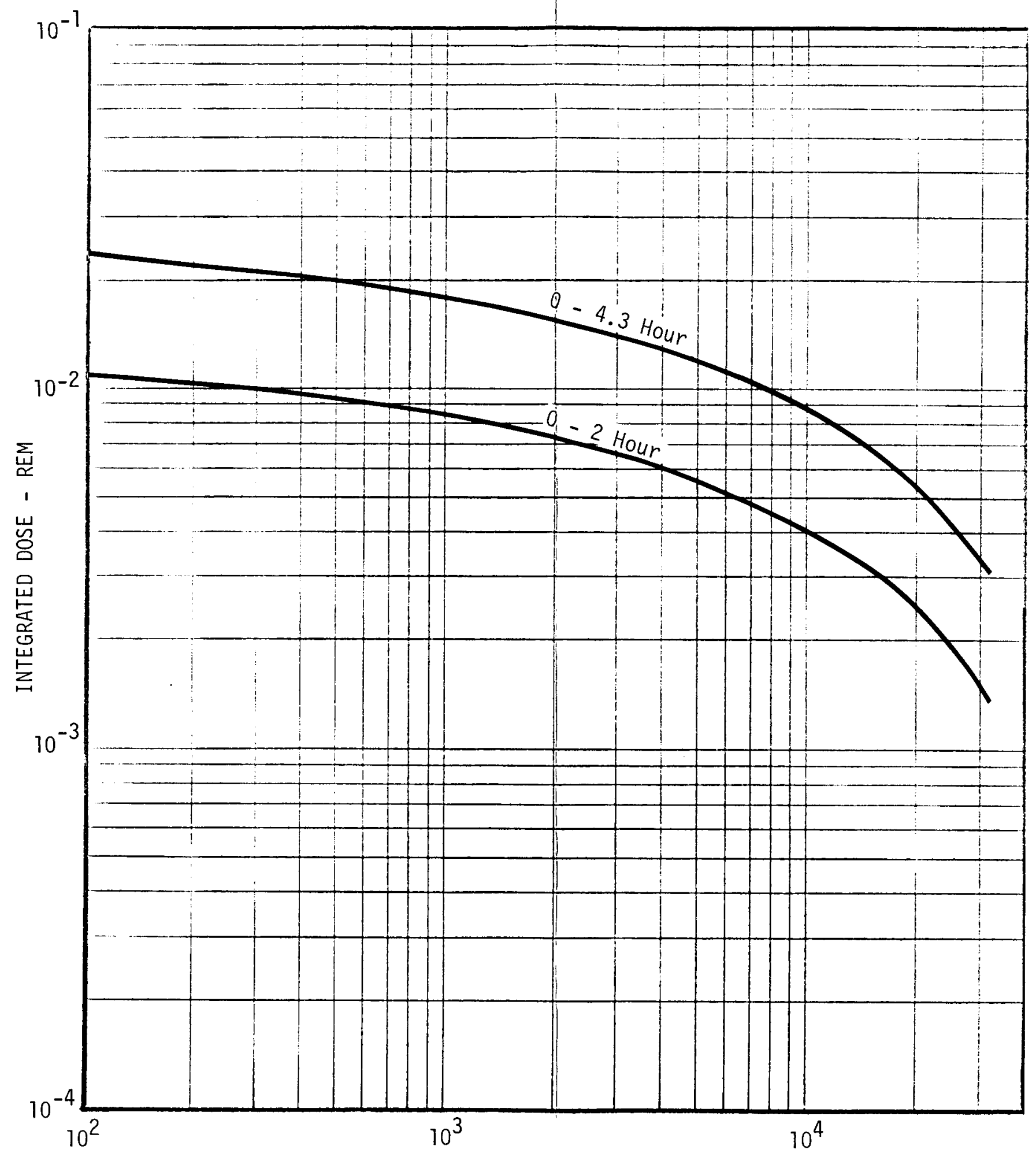

DISTANCE FROM STACK BASE-FEET

FIGURE 15.13 STEAM GENERATOR TUBE RUPTURE 
WIND SPEED: $4 \mathrm{MPH}$

DURATION OF ACCIDENT:

CONTAINMENT SPHERE ACCIDENT: 4 HOURS SPENT FUEL BUILDING ACCIDENT:1 HOUR

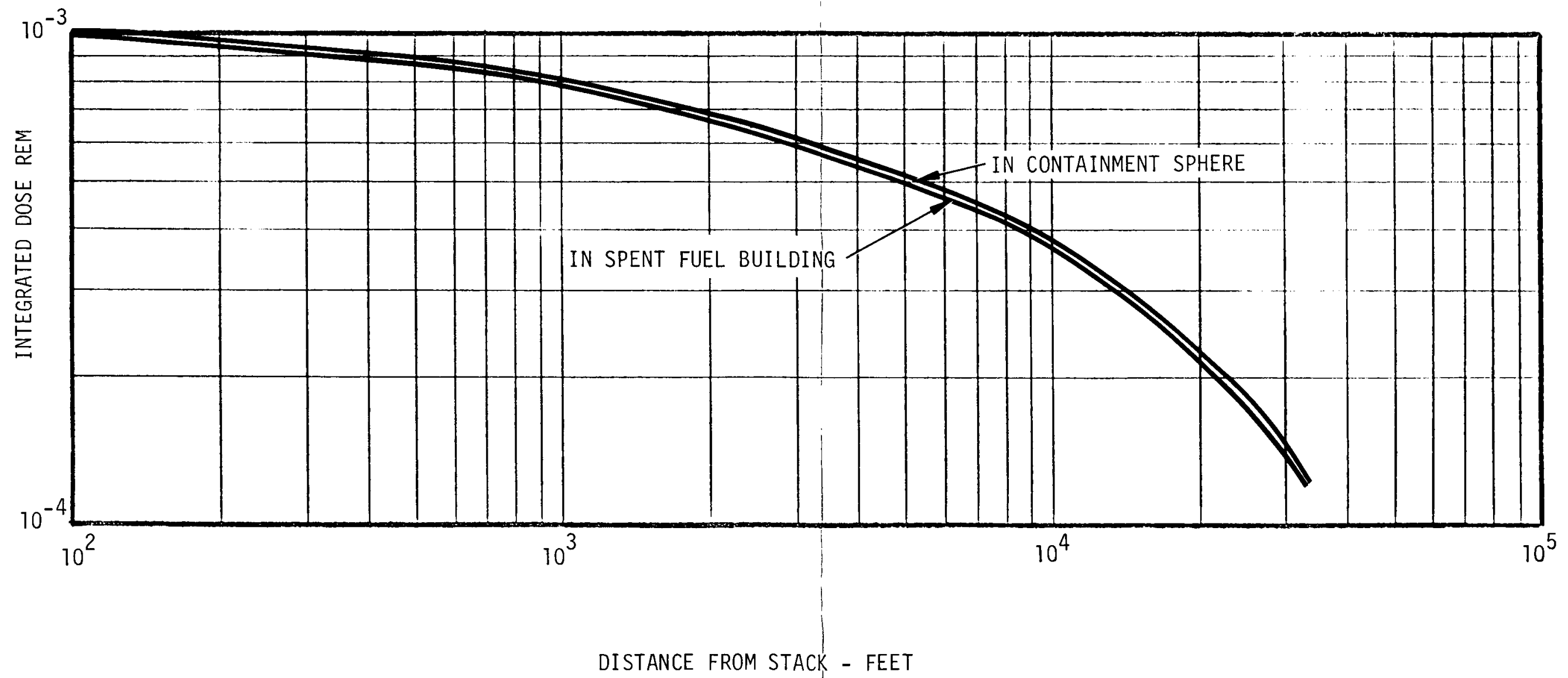

FIGURE 15.14 FUEL HANDLING ACCIDENT 


\subsubsection{ROD EJECTION ACCIDENT (Continued)}

same for this analysis. Figure 15.15 shows a whole-body dose due to direct radiation from activity retained in the containment.

Figures 15.16 and 15.17 show whole-body immersion doses for two cases of containment leakage, 0.5 percent per 24 hours by volume, and 1.9 percent per 24 hours by volume, respectively. In both cases the total integrated exposure is less than 25 rem.

Figures 15.18 and 15.19 for thyroid doses reflect exposure resulting from the two cases of containment leakage considered. In all cases, the zeroto-infinity exposure is less than $300 \mathrm{rem}$ beyond 900 feet from the containment centerline. 


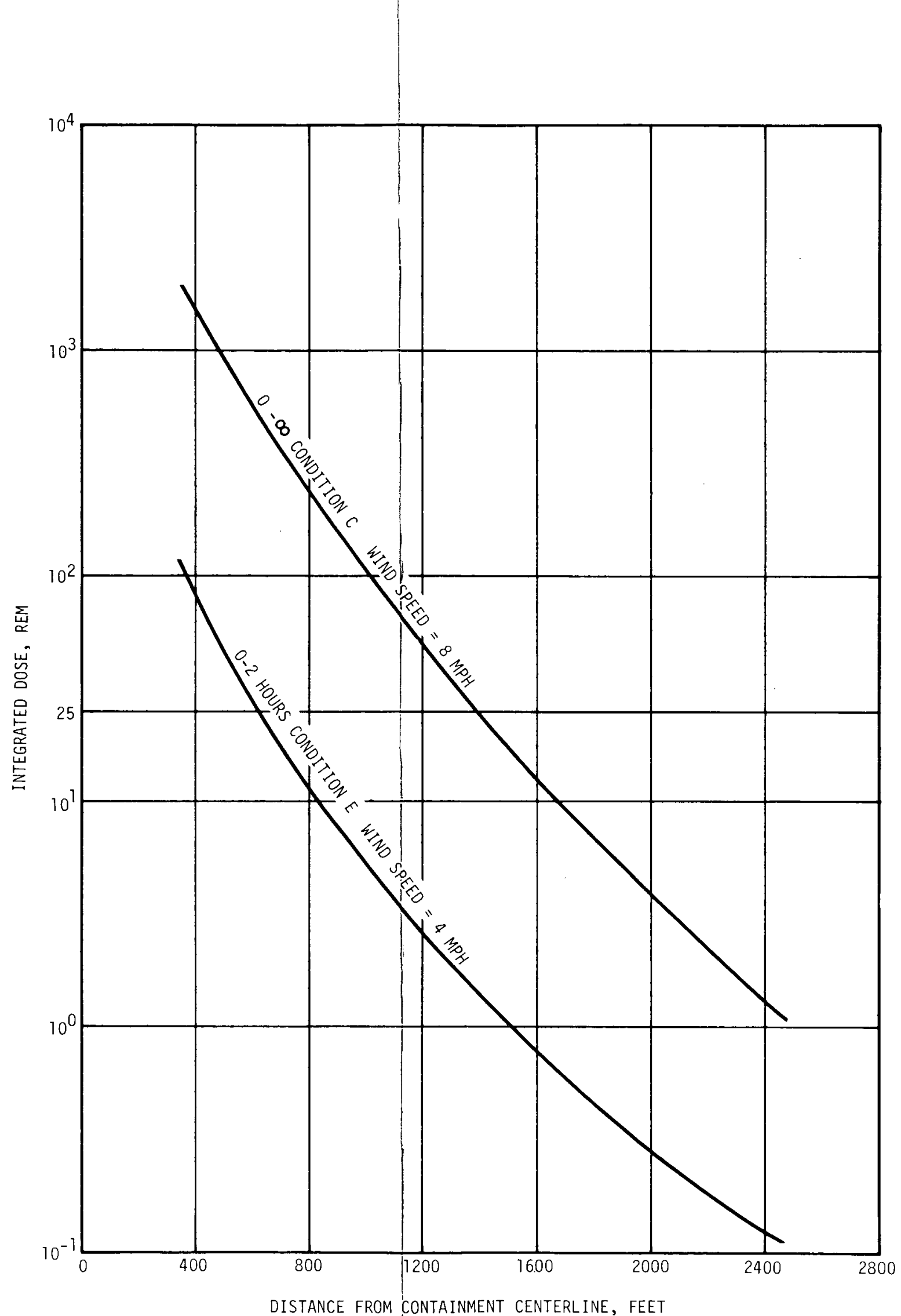

FIGURE 15.15

ROD EJECTION ACCIDENT, DIRECT DOSE 


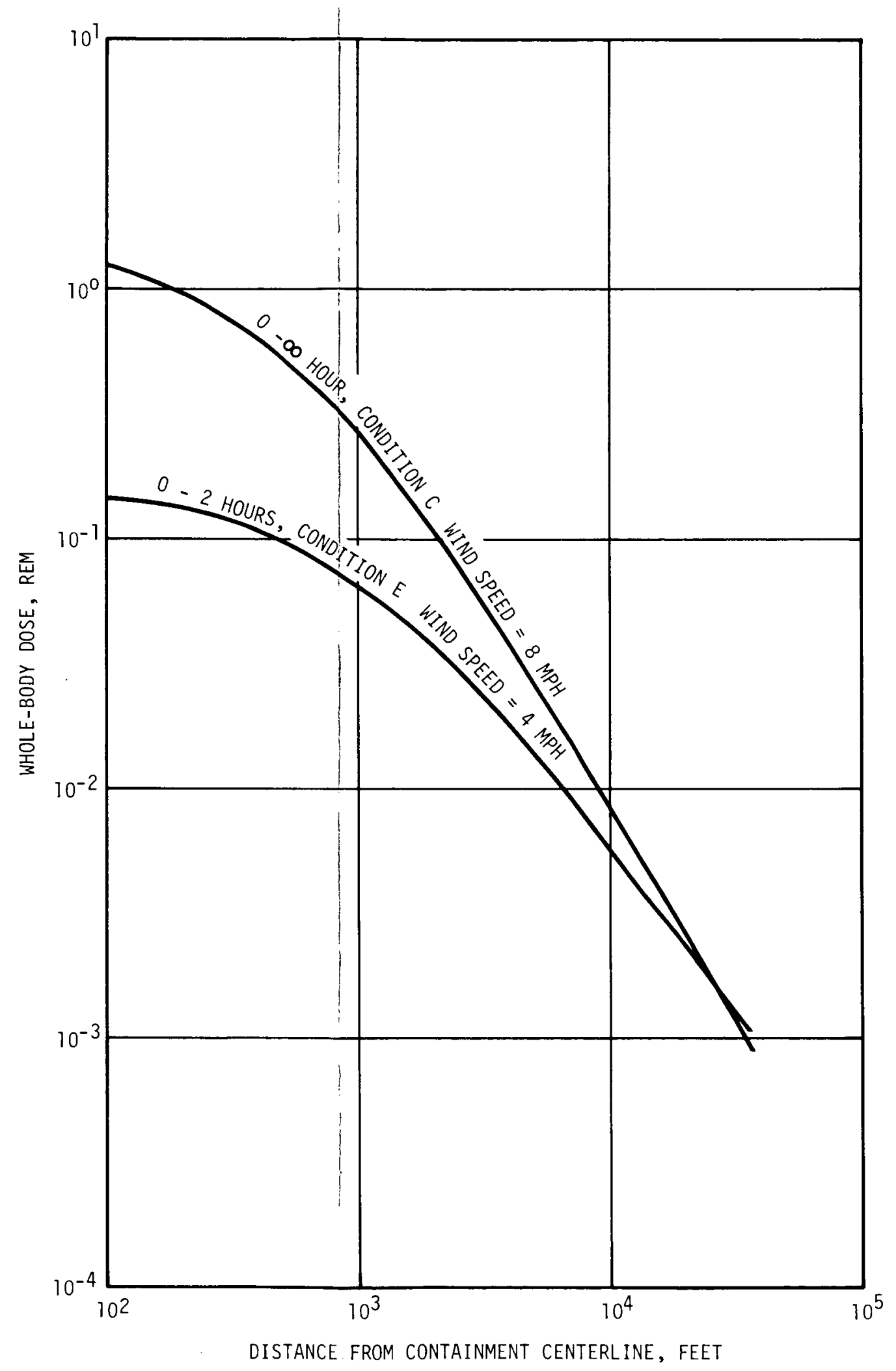

FIGURE 15.16

ROD EJECTION ACCIDENT, WHOLE-BODY IMMERSION DOSE, CONTAINMENT LEAK RATE OF 0.5 PERCENT PER DAY 


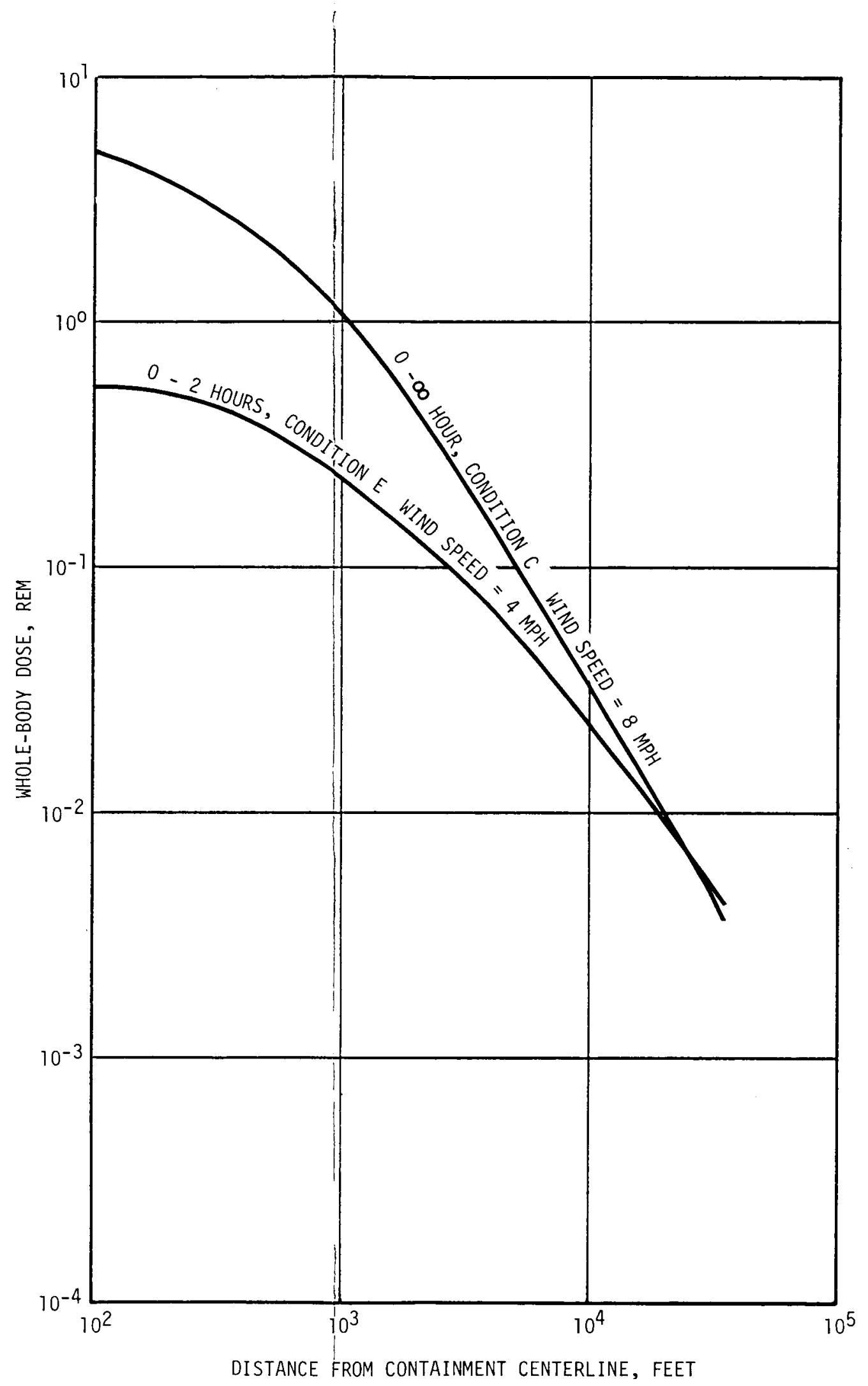

FIGURE 15.17

ROD EJECTION ACCIDENT, WHOLE-BODY IMMERSION DOSE, CONTAINMENT LEAK RATE OF 1.99 PERCENT PER DAY 


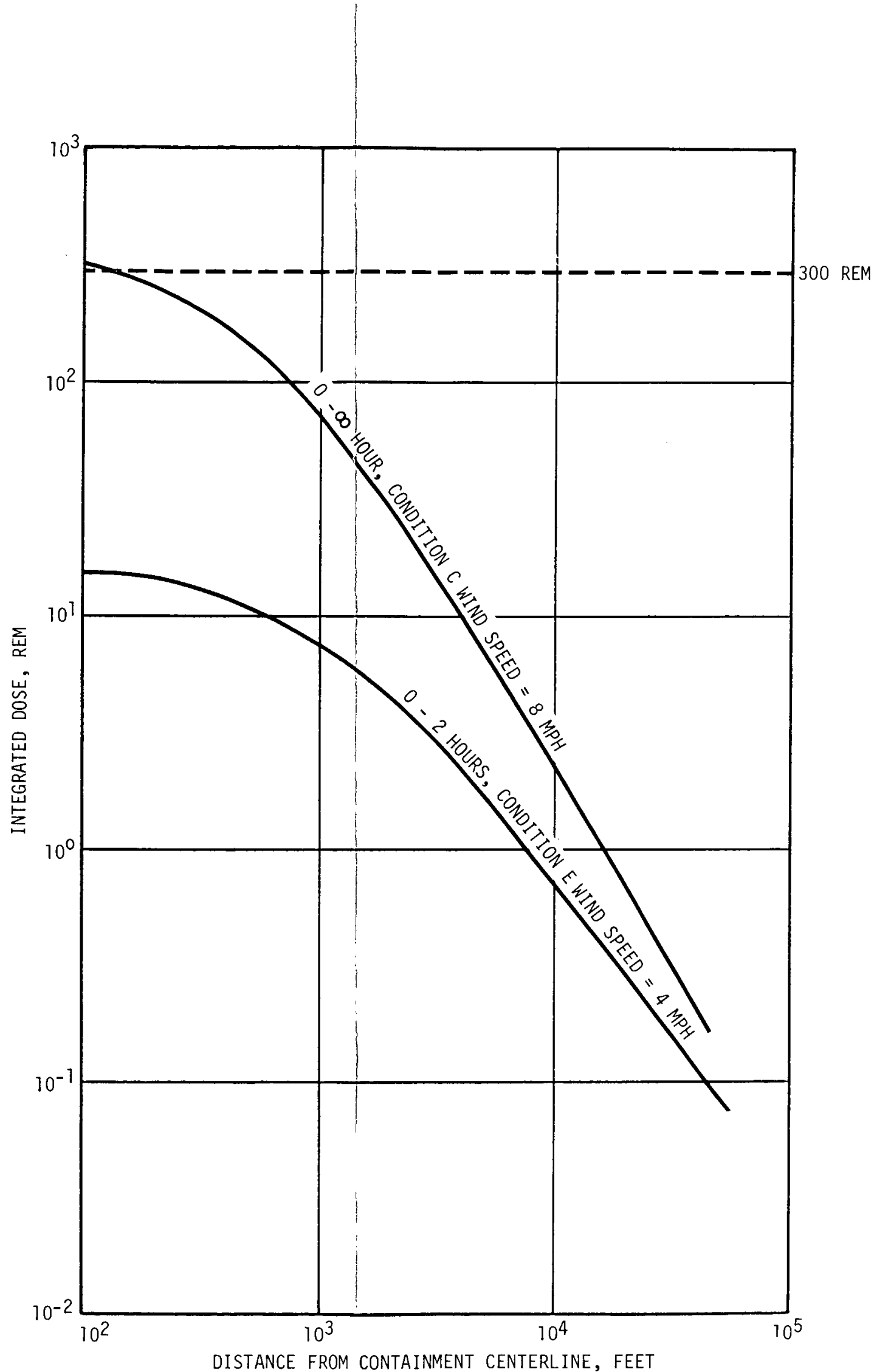

FIGURE 15.18 ROD EJECTION ACCIDENT, THYROID DOSE, CONTAINMENT LEAK RATE OF 0.5 PERCENT PER DAY 


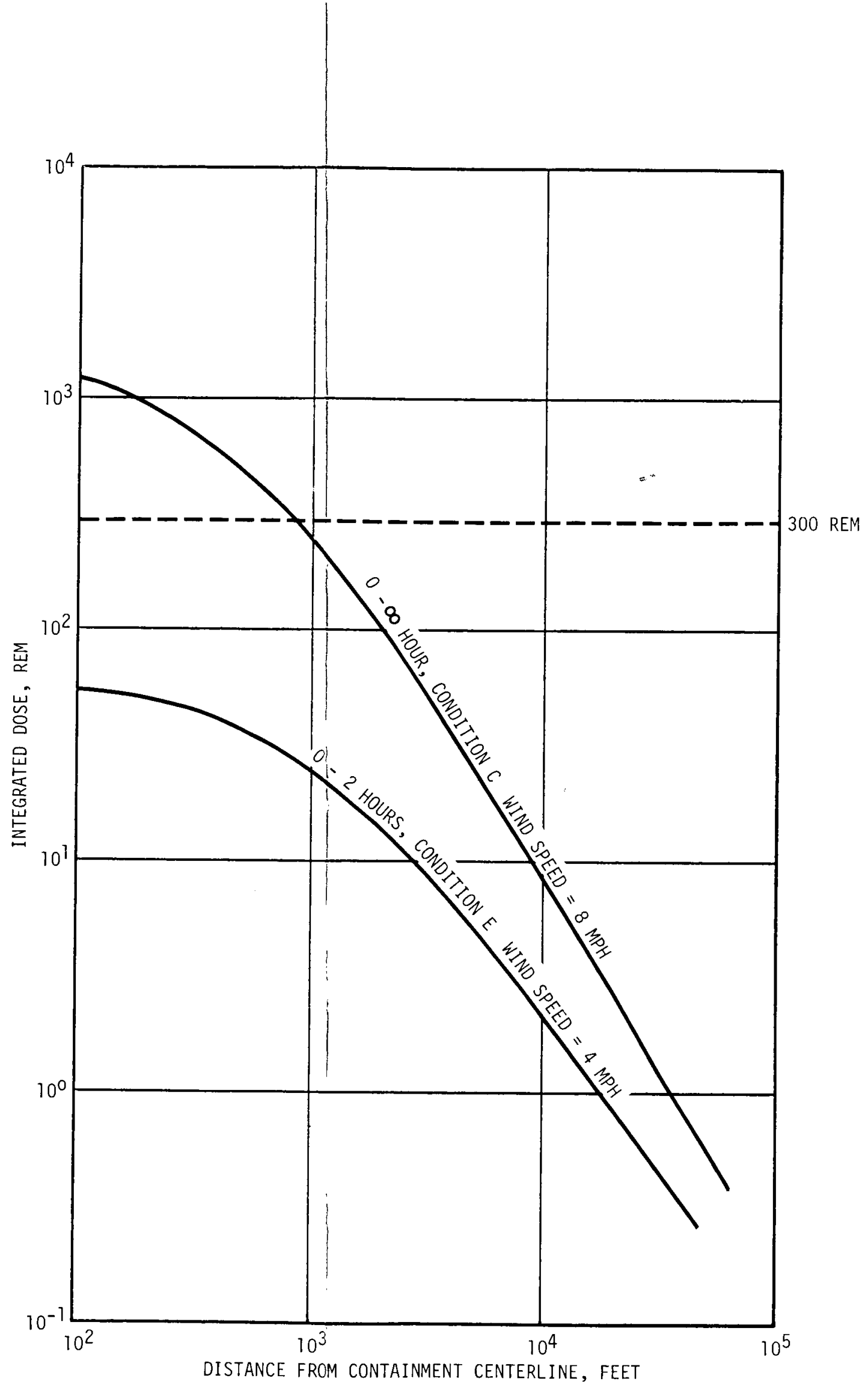

FIGURE 15.19 ROD EJECTION ACCIDENT, THYROID DOSE, CONTAINMENT LEAK RATE OF 1.9 PERCENT 


\section{SECTION 16 \\ CONCLUSIONS}

\subsection{CONCLUSIONS}

A pressurized water reactor of the type utilized at San Onofre possesses inherent stability characteristics and safety features. Extensive operating experience with this type of reactor has demonstrated the validity of these features, which include the following:

a. The Doppler coefficient in the slightly enriched $\mathrm{UO}_{2}$ fuel.

b. The overall effective negative temperature coefficient of the reactor system.

c. The four barriers (fuel, fuel cladding, Reactor Coolant System, Containment Sphere) against inadvertent release of fission products to the environment.

The features have been extensively analyzed and discussed in this report, and their adequacy demonstrated under various postulated conditions.

The inherent safety features in the nuclear design of the station provide a system with self-limiting capabilities in the unlikely event of a reactor transient. Engineered safeguards are also provided to detect, control, and eliminate the possibility of a nuclear incident occurrence. In the unlikely event such an incident should occur, the safeguards would limit any consequences to acceptable values. These safeguards consist of:

a. Safety Injection System - In the event of a rupture of the largest reactor coolant system piping (2-1/2 inch-thick wall, 27-1/2 inches ID), the Safety Injection System will provide an immediate supply of borated water to cover and protect the core. This controls the initial fission product release from the core and prevents any further release of fission products.

b. Recirculation System - If required after any assumed accident, the recirculation system provides long-term cooling of the core for any length of time desired.

c. Containment Sphere - For any postulated accident where reactor coolant is released, the high integrity, essentially leak-tight sphere serves as a container for the thermal energy and fission products released from the coolant. In addition, the single shell steel sphere promotes a significant amount of heat transfer to atmosphere for dissipation of thermal energy.

d. Containment Spray Systein - Spray systems in both the upper portion of the sphere and upper inside portion of the secondary shield, 


\subsection{CONCLUSIONS (Continued)}

provide significant heat removal and fission product washdown capability.

Where applicable, the safeguard systems have been provided with duplicate, redundant equipment and controls to assure their satisfactory operation.

Although not classified as a safeguard system, the Radioactive Waste Disposal system serves a similar function. During normal operation of the station, the system processes, monitors, stores, and discharges, as required, any radioactive wastes produced in the station. Discharge of wastes is under controlled conditions, and maintained within the levels established by federal, state, and local regulatory agencies.

In order to obtain assurance that the various safety features and safeguard systems will serve their design function, numerous postulated accidents, however unlikely, have been analyzed to evaluate the consequences. The analyses demonstrate the adequacy of the safeguard systems, and show the high degree of safety incorporated into the station.

In addition, supervisory and operating personnel for the station were carefully selected, with major consideration given to previous experience in power plant operation. Extensive special training in nuclear reactor operation has resulted in a highly competent and capable operating staff.

Based upon the extensive analysis that was performed during design, and after careful consideration of all aspects of the facility, the applicants believe that the San Onofre Nuclear Generating Station, Unit 1, will be beneficial to the public in the development of peaceful uses of atomic energy, and, in accordance with 10CFR50, "will not endanger public health and safety". 


\section{APPENDIX A}

GENERAL ASSUMPTIONS FOR ACCIDENT ANALYSES

\section{REACTOR TRIP}

A reactor trip signal acts to open the two series trip breakers feeding power to the control rod drive mechanisms. The loss of power to the mechanism coils causes the mechanisms to release the control rods which are then inserted, by gravity fall, into the core. There are various instrumentation delays associated with each tripping function including delays in signal actuation, in opening the trip breakers, and in the release of the rods by the mechanisms. The total delay to trip is defined as the time delay from the time the signal reaches the trip level, depending on the particular transient, to the time the rods are free and begin to fall. The time delay assumed for each tripping function is given in Table A-1.

Table A.1 Assumed Time Delays

\begin{tabular}{|c|c|}
\hline Tripping Function & Time Delay (Seconds) \\
\hline Startup rate & 3.5 \\
\hline Overpower (nuclear) & 0.32 \\
\hline $\begin{array}{l}\text { Variable low pressure } \\
\text { (includes delay in } \\
\text { temperature detectors) }\end{array}$ & 2.82 \\
\hline High pressurizer pressure & 2.0 \\
\hline High pressurizer level & 2.0 \\
\hline $\begin{array}{l}\text { Low reactor coolant flow } \\
\text { (from loop flow detectors) }\end{array}$ & 0.8 \\
\hline
\end{tabular}

The reactivity insertion following a reactor trip is a function of the acceleration of the control rods and the variation in rod worth as a function of rod position. The control rod position is determined experimentally as a function of time in a simulated reactor trip using an actual prototype assembly under simulated flow conditions. Control rod position versus time is shown in Figure A.1. Reactor coolant flow simulated for this case was conservatively set at 120 percent of normal flow.

Control rod reactivity insertion as a function of rod position in the core is shown in Figures A.2 through A.3 in terms of percentage of total worth. This function is shown for both hot zero power and hot full power conditions. These functions when combined with the position versus time function 


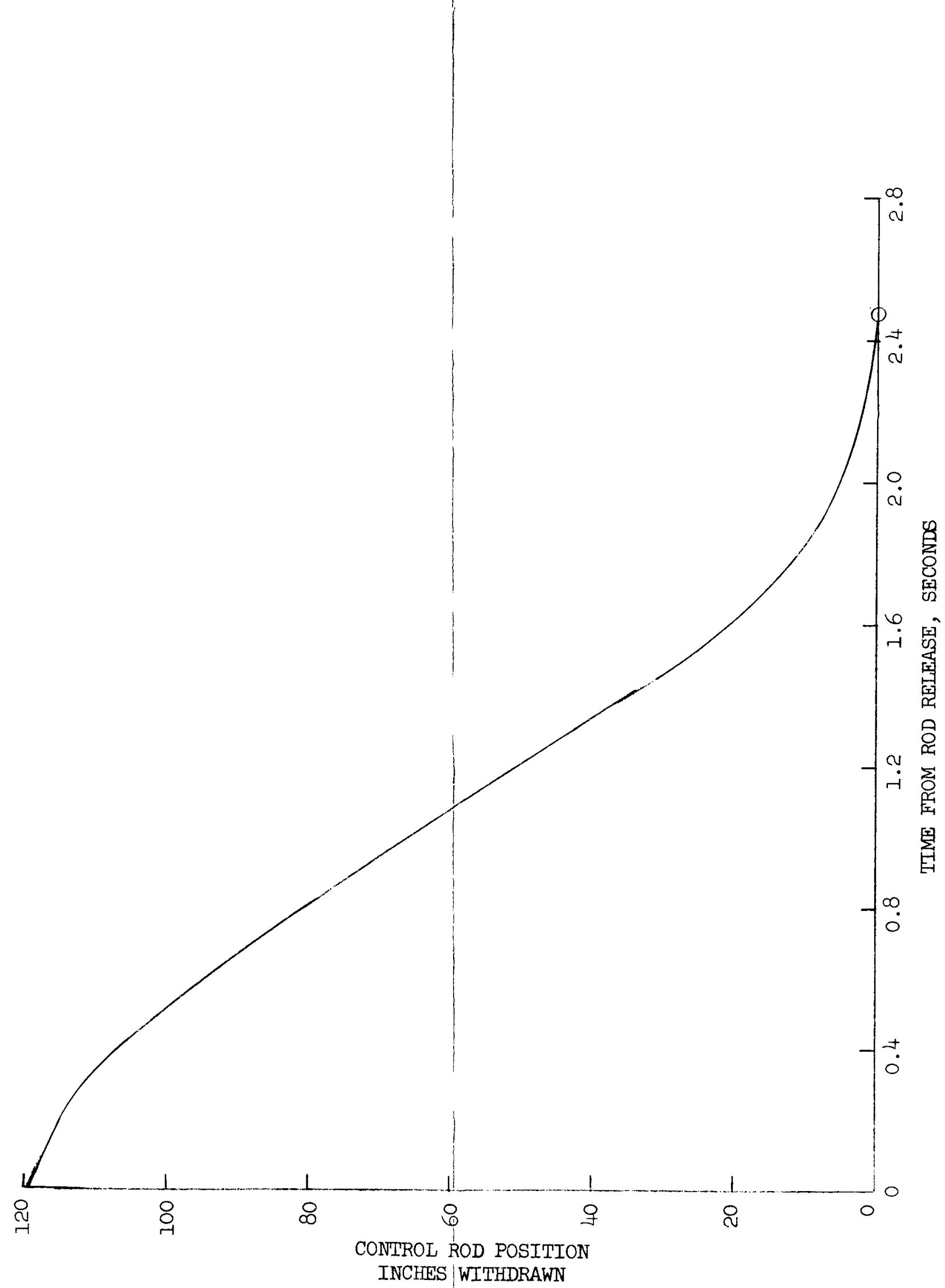

FIGURE A.1 CONTROL ROD POSITION VERSUS TIME FOR REACTOR TRIP WITH 120 PERCENT REACTOR COOLANT FLOW 


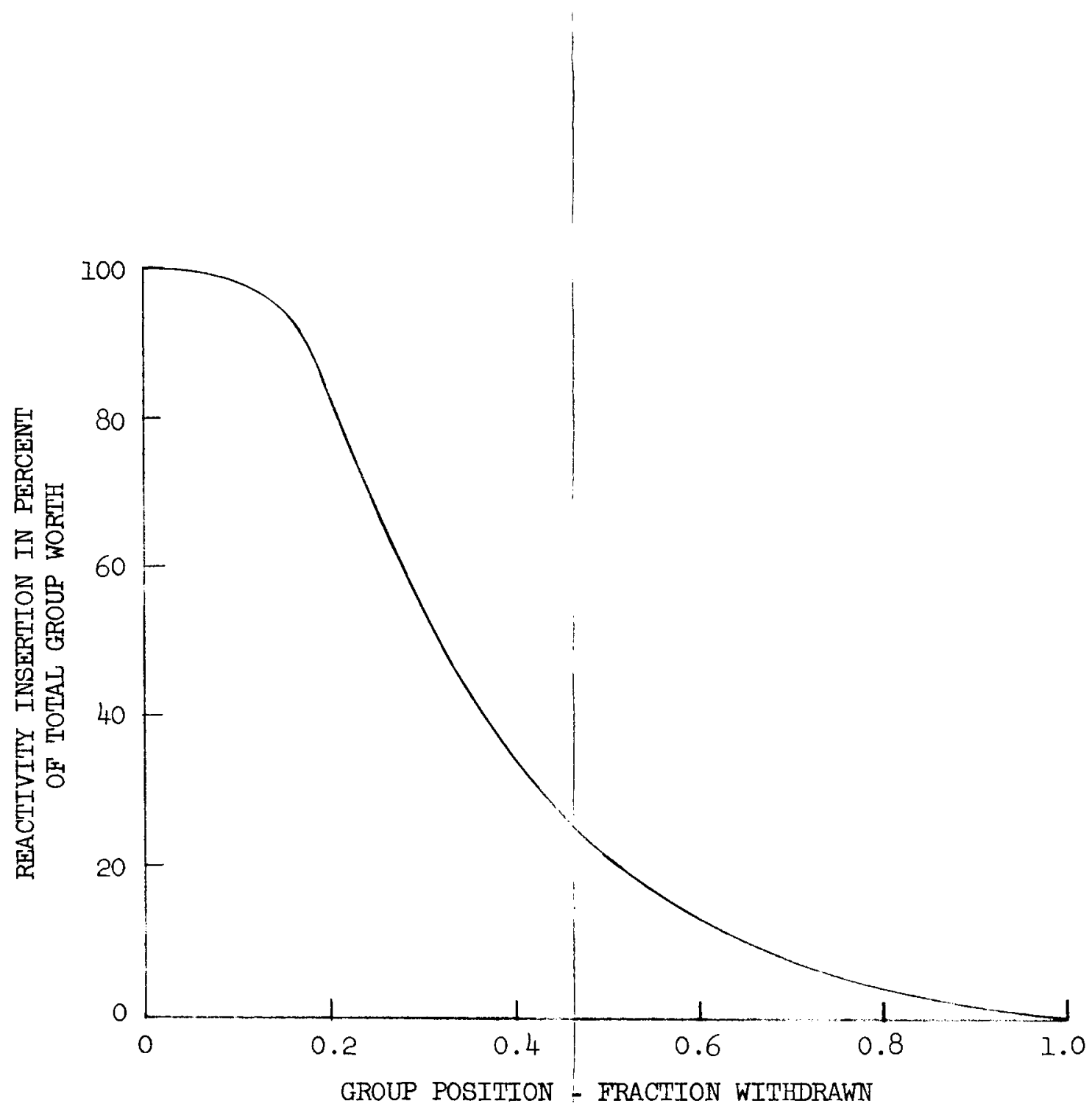

FIGURE A.2 CONTROL ROD REACTIVITY INSERTION VERSUS ROD POSITION FOR HOT ZERO POWER CONDITIONS 


\section{REACTOR TRIP (Continued)}

given in Figure A.I result in the reactivity insertion versus time functions shown in Figures A.4 and A.5. The zero power function was conservatively applied for all reactor trips since this results in the longest delay before an effective trip.

\section{HOT CHANNEI CONDITIONS}

Unless stated otherwise in the sections describing specific accident conditions, the margins to DNB were determined on the basis of design hot channel factors $F_{a}^{E}$ (heat $f l u x$ engineering rise hot channel factor) $=3.23$ and $\mathrm{F}_{\triangle \mathrm{H}}^{\mathrm{E}}$ (enthalpy rise engineering hot channel factor) $=1.88$. The conservative basis for the various subfactors which make up these total factors are presented in Paragraphs 3.3 and 3.4. As shown in Paragraph 3.4 the computed nuclear hot channel factors are substantially below the design objectives consistent with the total factors given above. The in-core instrumentation system will be employed to check the initial power distributions and to follow the power distribution throughout core lifetime. The axial flux distribution is conservatively assumed to be an extended cosine distribution with a maximum to average ratio of 1.78 . 


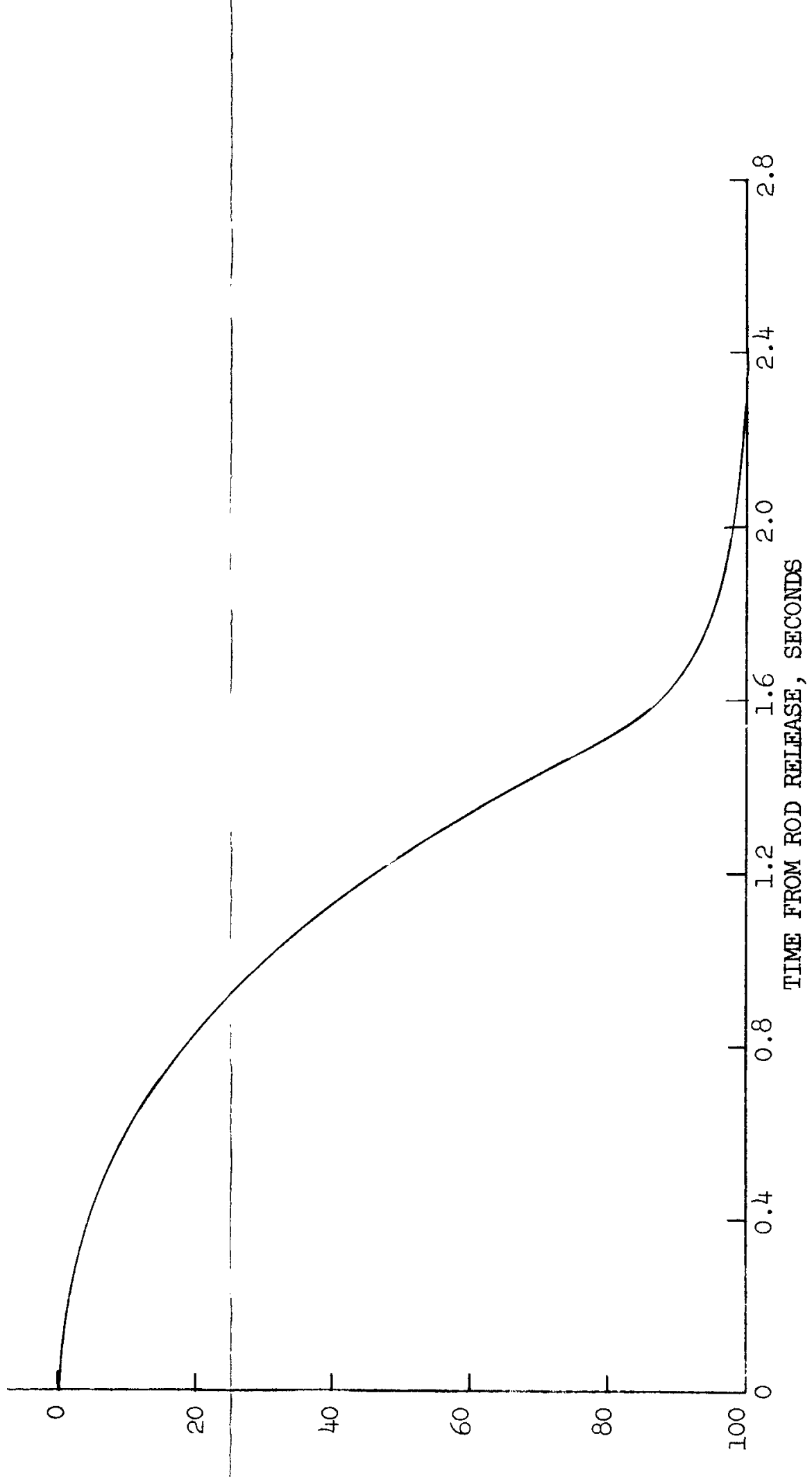

REACTIVITY INSERTION

PERCENT OF TOTAL WORTH

FIGURE A.5

REACTIVITY INSERTION VERSUS TIME FOR REACTOR TRIP, HOT FULL POWER CONDITIONS, 120 PERCENT REACTOR COOLANT FLOW 


\section{APPENDIX B}

DRAWING SYMBOLS AND ABBREVIATIONS

This appendix has been included to provide drawing symbols and abbreviations used throughout the report. Drawing symbols are shown in Figure B.1. An abbreviation list begins on Page B-3. 


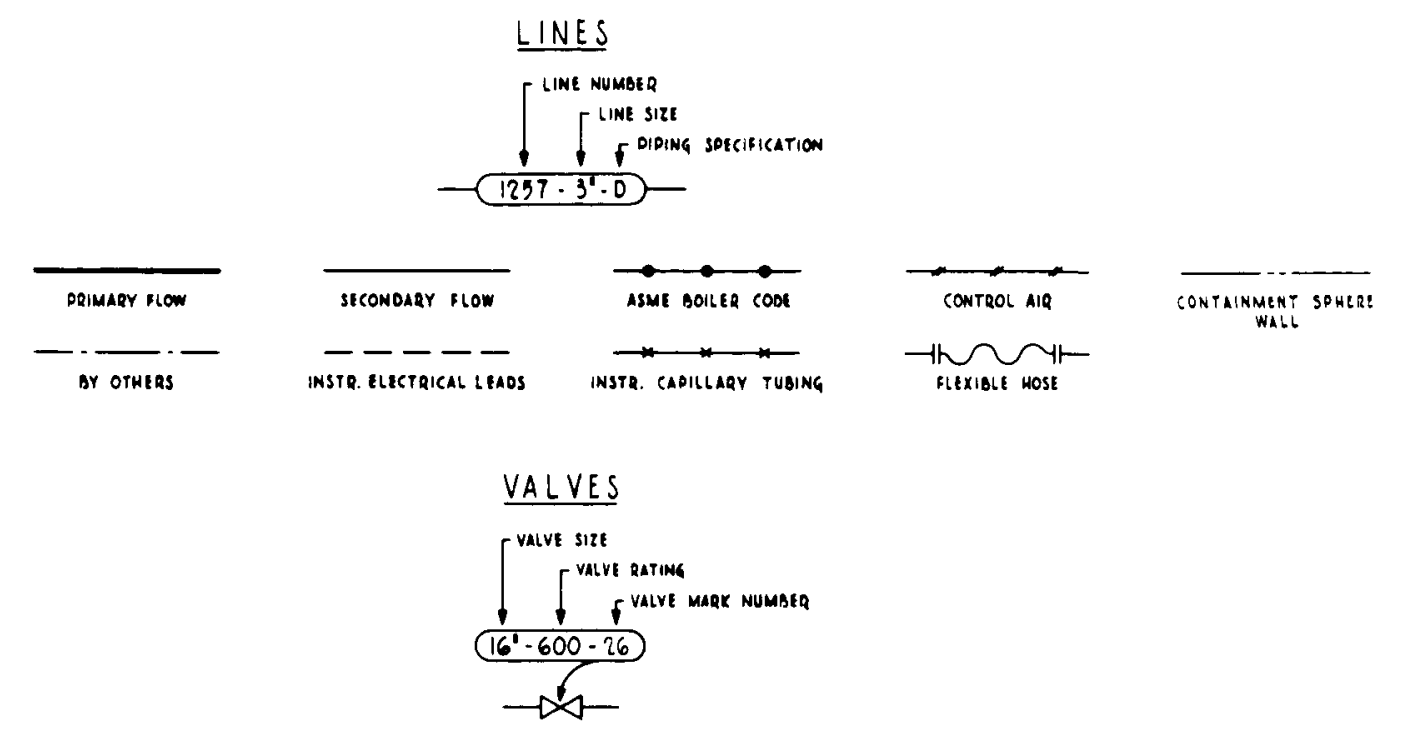

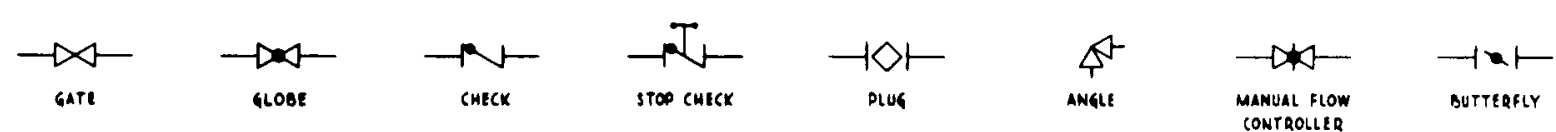

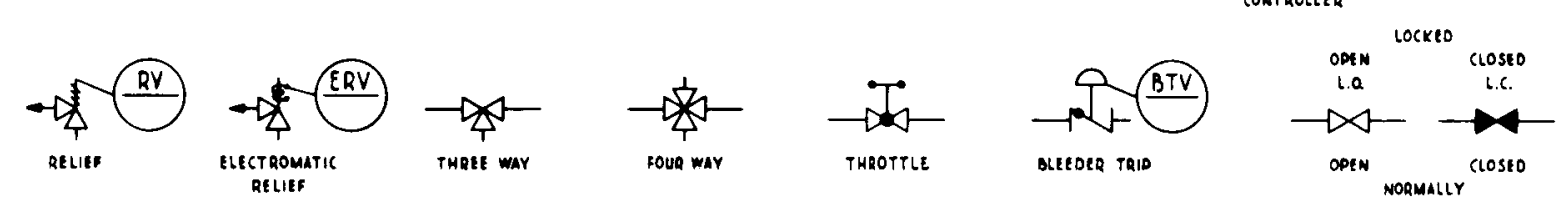

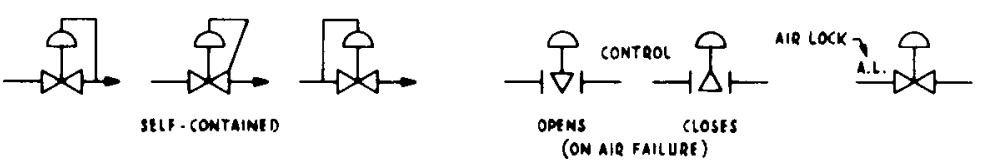

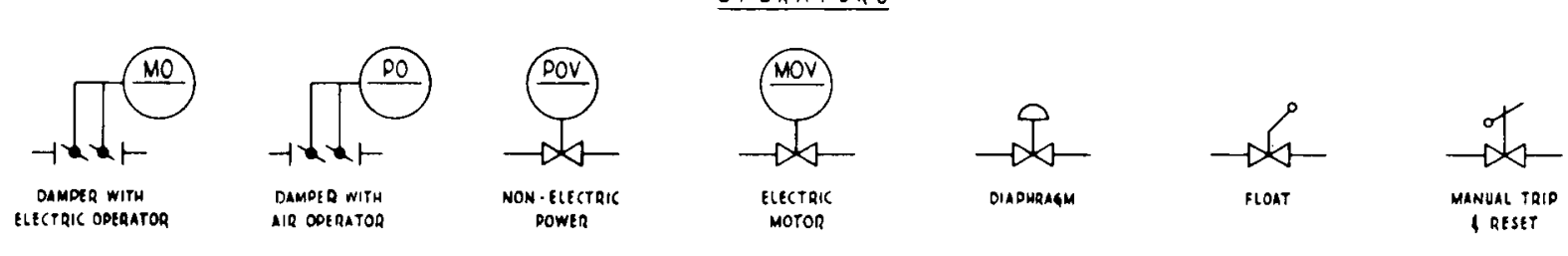

$\rightarrow-(S$

INSTRUMENTS

$$
\text { LOCAL MOUNTED }
$$

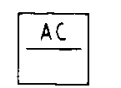

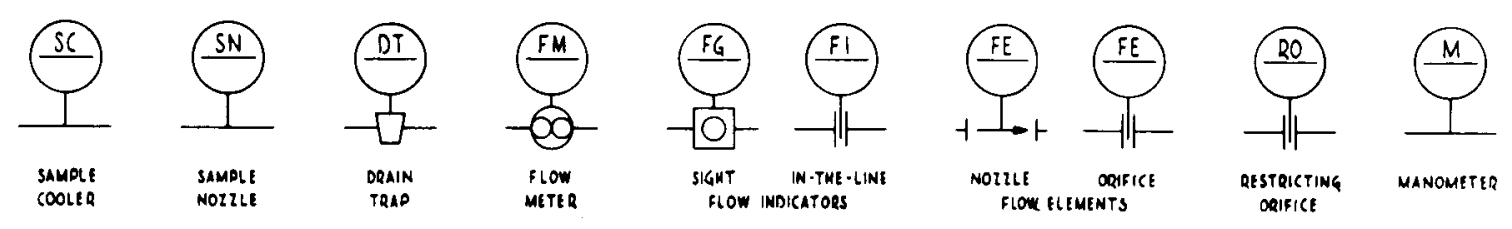

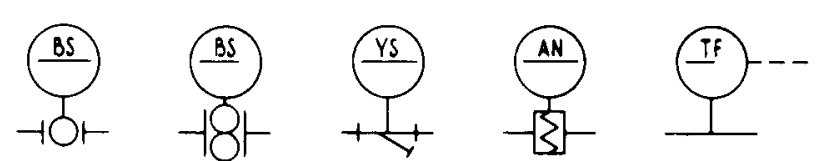

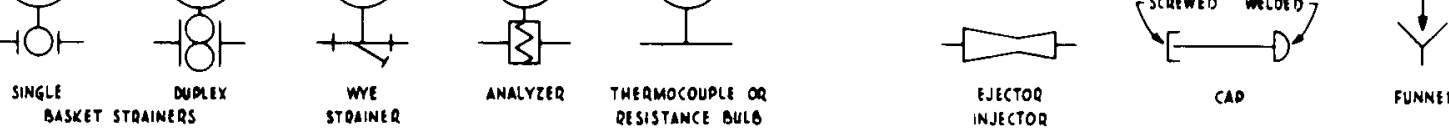

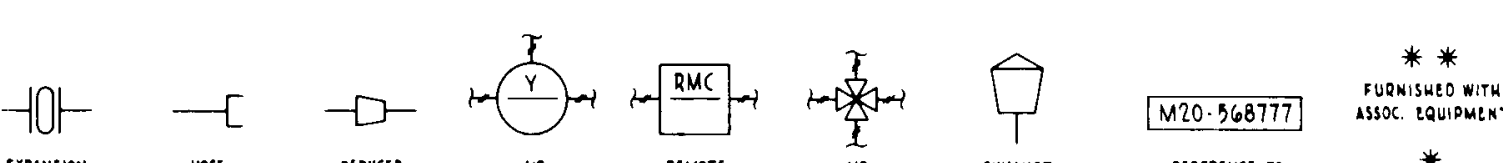

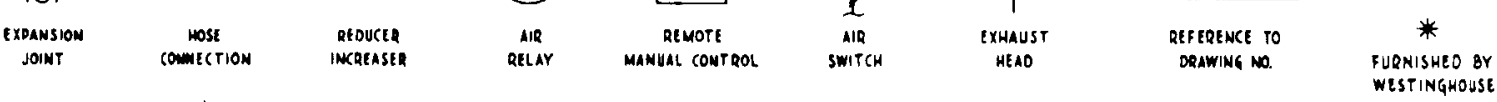

\rangle$_{1}^{-10}$

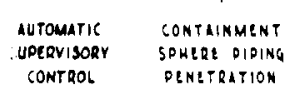

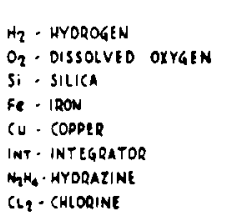

INSTRUMENT IDENTIFICATIONS

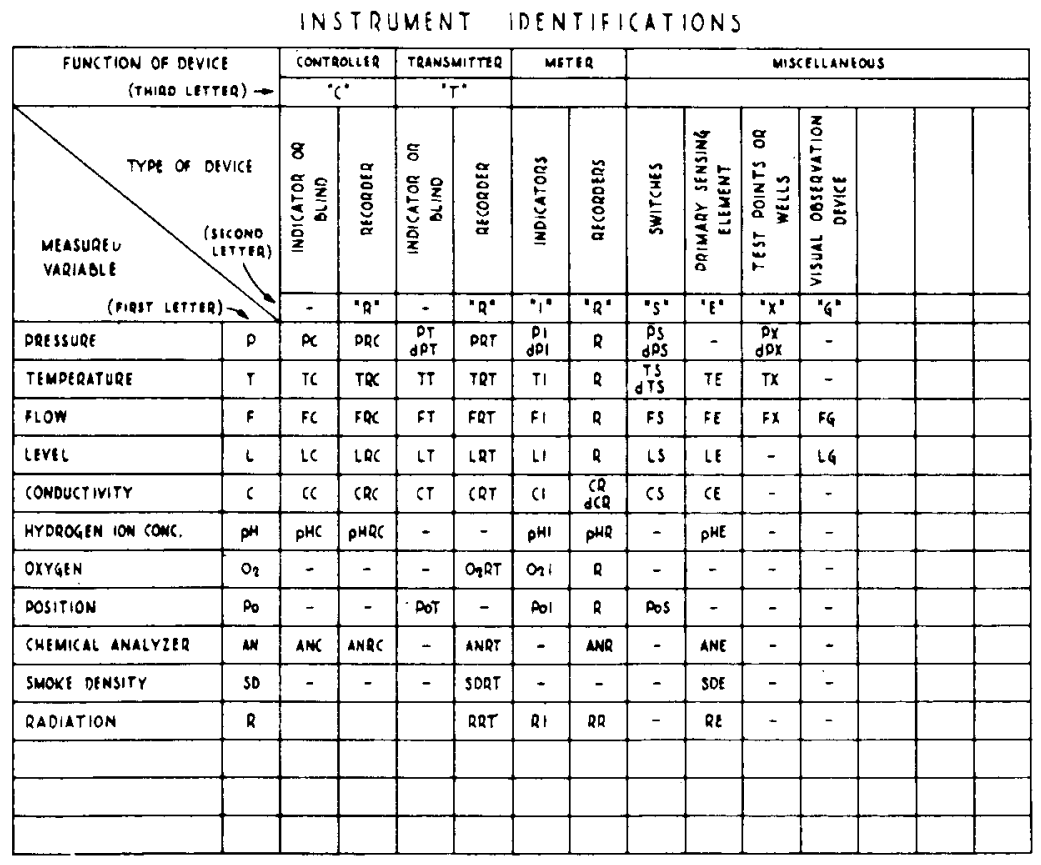

SELF CONTAINED VALVES

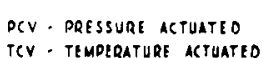

tuberature acio

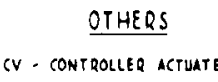


ABBREVIATIONS

\begin{tabular}{|c|c|c|c|}
\hline $\begin{array}{l}\text { A } \\
\text { abamp } \\
\text { abs }\end{array}$ & $\begin{array}{l}\text { angstrom } \\
\text { absolute ampere } \\
\text { absolute (unit) }\end{array}$ & $\begin{array}{l}\text { ch } \\
\text { c-hr } \\
\text { chem }\end{array}$ & $\begin{array}{l}\text { chapter } \\
\text { candle-hour } \\
\text { chemical }\end{array}$ \\
\hline ac & alternating current (noun) & cif & cost, insurance, and freight \\
\hline$a-c$ & alternating current (adj) & cir & circular \\
\hline $\mathrm{AEC}$ & Atomic Energy Commission & $\mathrm{cm}$ & centimeter \\
\hline $\mathrm{AF}$ & audio frequency & $\mathrm{cm}^{3}$ & cubic centimeter (volume) \\
\hline a.m. & before noon & $\mathrm{cn}$ & cosine of the amplitude \\
\hline $\mathrm{AM}$ & amplitude modulation & $\operatorname{coef}$ & coefficient \\
\hline amp & ampere & $\operatorname{col}$ & column \\
\hline$a m p-h r$ & ampere-hour & $\operatorname{colog}$ & cologarithm \\
\hline amu & atomic mass unit & conc & concentrate, -tion \\
\hline$A_{S}$ & atmosphere, standard & cond & conductivity \\
\hline atm & atmosphere & const & constant \\
\hline at. wt & a tomic weight & cont hp & continental horsepower \\
\hline at. $\%$ & atomic percent & $\cos$ & cosine \\
\hline av & average & $\begin{array}{l}\cosh \\
\cot \end{array}$ & $\begin{array}{l}\text { cosine, hyperbolic } \\
\text { cotangent }\end{array}$ \\
\hline $\mathrm{b}$ & barn & $\mathrm{cp}$ & chemica $\downarrow$ ly pure \\
\hline ba 1 . & ba lance & $\mathrm{cP}$ & centipoise \\
\hline bar. & barometer & cpd & contact potential difference \\
\hline bbl & barre 1 & cpm & cycles per minute \\
\hline bcc & body-centered cubic & cps & cycles per second \\
\hline $\mathrm{Be}$ & Baume & crit & critical \\
\hline bev & billion electron volts & $\csc$ & cosecant \\
\hline bhp & brake horsepower & $\operatorname{csch}$ & hyperbolic cosecant \\
\hline binp-hr & brake horsepower-hour & c to $c$ & center-to-center \\
\hline bmep & brake mean effective pressure & $\mathrm{cu}$ & cubic \\
\hline bp & boiling point & $\mathrm{cu} \mathrm{ft}$ & cubic foot \\
\hline bpd & barrels per day & $\mathrm{cu}$ in. & cubic inch \\
\hline Btu & British thermal unit & cu yd & cubic yard \\
\hline bu & bushe 1 & $\mathrm{cw}$ & continuous wave \\
\hline BWR & boiling water reactor & $\begin{array}{l}\text { cwt } \\
\text { cy }\end{array}$ & $\begin{array}{l}\text { hundredweight } \\
\text { cycle }\end{array}$ \\
\hline c & curie & $\operatorname{cyl} 1$ & cylinder \\
\hline c. & copyright & & \\
\hline \& & cent & $\mathrm{dc}$ & direct current (noun) \\
\hline $\mathrm{C}$ & $\begin{array}{l}\text { degrees centigrade, } \\
\text { also hundred }\end{array}$ & $\begin{array}{l}d-c \\
d e g\end{array}$ & $\begin{array}{l}\text { direct current (adj) } \\
\text { degree, angular }\end{array}$ \\
\hline ca. & about (circa) & diam & diameter \\
\hline ca 1 & calorie & dis/sec & disintegrations per second \\
\hline $\mathrm{calc}$ & calculated & $\mathrm{dn}$ & delta amplitude \\
\hline $\begin{array}{l}\text { Calif. } \\
\mathrm{cc}\end{array}$ & $\begin{array}{l}\text { California } \\
\text { cubic centimeter (liquid) }\end{array}$ & DNB & $\begin{array}{l}\text { departure from nucleate } \\
\text { boiling }\end{array}$ \\
\hline$c d$ & candela & doz & dozen \\
\hline $\operatorname{cemf}$ & counter electromotive force & $\mathrm{dr}$ & dram \\
\hline cf. & compare & $d w t$ & deadweight ton \\
\hline cfh & cubic feet per hour & & \\
\hline $\mathrm{cfm}$ & cubic feet per minuite & $\mathrm{E}$ & east \\
\hline CFR & Code of Federal Regulations & eap & equivalent air pressure \\
\hline cfs & cubic feet per second & ehp & effective horsepower \\
\hline $\mathrm{cg}$ & centigram & eff & efficiency \\
\hline CG & center of gravity & e.g. & for example \\
\hline
\end{tabular}




\begin{tabular}{|c|c|c|c|}
\hline e 1 & elevation & $h-p$ & high-pressure (adj) \\
\hline elec & electric, -al, -ity & hp-hr & horsepower-hour \\
\hline elhp & electric horsepower & $\mathrm{hr}$ & hour \\
\hline emf & electromotive force & hwy & highway \\
\hline emu & electromagnetic unit & & \\
\hline eq & equation & IA & international angstrom \\
\hline esu & electrostatic unit & ibid. & in the same place \\
\hline et a 1 . & and others & ICT & International Critical Tables \\
\hline etc. & and so forth & ID & inside diameter \\
\hline eu & entropy unit & ie. & that is \\
\hline ev & electron volt & IF & intermediate frequency \\
\hline evap & evaporation, -tive & ifc & indicated fuel consumption \\
\hline $\exp$ & exponential & ihp & indicated horsepower \\
\hline ext & external & imep & $\begin{array}{l}\text { indicated mean effective } \\
\text { pressure }\end{array}$ \\
\hline $\mathrm{f}$ & farad & in. & inch \\
\hline $\mathrm{F}$ & degrees Fahrenheit & Inc. & incorporated (as a title) \\
\hline fas & free a longside ship & in. $\mathrm{Hg}$ & inches of mercury \\
\hline fhp & friction horsepower & in. $-1 b$ & inch-pound \\
\hline fig. & figure & int & interna 1 \\
\hline $\mathrm{E} 1$ & fluid & ipm & inches per minute \\
\hline FM & frequency modulation & ips & inches per second \\
\hline fmep & friction mean effective & IPS & iron pipe size \\
\hline & pressure & isoth & isotherm, -a 1 \\
\hline fob & free on board & itd & initial temperature difference \\
\hline $\mathrm{fp}$ & freezing point, fusion point & & \\
\hline fpm & feet per ininute & j & joule \\
\hline fps & feet per second & & \\
\hline $\mathrm{Fr}$ & franklin & $\mathrm{kc}$ & kilocurie \\
\hline $\mathrm{ft}$ & foot & $\mathrm{KC}$ & kilocycles per second \\
\hline $\mathrm{ft}-\mathrm{c}$ & foot-candle & $\mathrm{kcal}$ & kilocalorie \\
\hline$f t-L$ & foot-lambert & ke & kinetic energy \\
\hline$f t-1 b$ & foot-pound & kev & kilo electron volts \\
\hline$f t-\sec ^{2}$ & feet per second per second & $\mathrm{kg}$ & ki logram \\
\hline & & $\mathrm{kG}$ & kilogauss \\
\hline$g$ & gram, force or gravity & $\mathrm{kg}-\mathrm{ca} 1$ & kilogram-calorie \\
\hline G & gauss & $\mathrm{kg}-\mathrm{m}$ & kilogram-meter \\
\hline ga 1 & gallon & $\mathrm{kg} / \mathrm{m}^{3}$ & kilograms per cubic meter \\
\hline$g-c a l$ & gram-calorie & kgps & kilograms per second \\
\hline gcd & greatest common divisor & $\mathrm{kg}-\mathrm{wt}$ & kilogram-weight \\
\hline Gi & gilbert & kip & thousand $1 \mathrm{~b}$ \\
\hline gpd & gallons per day & $k i p-f t$ & thousand $\mathrm{ft}-\mathrm{lb}$ \\
\hline $\mathrm{gph}$ & gallons per hour & $\mathrm{kj}$ & kilojoule \\
\hline $\mathrm{gpm}$ & gallons per minute & $\mathrm{k} 1$ & kiloliter \\
\hline gps & gallons per second & $\mathrm{km}$ & kilometer \\
\hline$g r$ & grain, gross & kmps & kilometers per second \\
\hline & & $\mathrm{kOe}$ & kilo-oersted \\
\hline $\mathrm{h}$ & henry & ksi & kips per square inch \\
\hline ha & hectare & $k t$ & kiloton \\
\hline hav & haversine & $\mathrm{kv}$ & kilovolt \\
\hline $\mathrm{HF}$ & high frequency & kva & kilovolt-ampere \\
\hline hfs & hyperfine structure & kvar & reactive kilovolt ampere \\
\hline hhv & high heat value & $\mathrm{kW}$ & kilowatt \\
\hline hp & horsepower & kwhr & kilowatt-hour \\
\hline
\end{tabular}




\begin{tabular}{|c|c|c|c|}
\hline $\mathrm{k} \Omega$ & kilohm & $\begin{array}{l}\text { mhep } \\
\text { min }\end{array}$ & $\begin{array}{l}\text { mean horizontal candlepower } \\
\text { minimum }\end{array}$ \\
\hline 1 & liter & $\operatorname{mip}$ & mean indicated pressure \\
\hline $\mathrm{L}$ & lambert & $\mathrm{m}-\mathrm{kg}$ & meter-kilogram \\
\hline lat & latitude & $\mathrm{ml}$ & milliliter \\
\hline $1 \mathrm{~b}$ & pound & $\mathrm{mL}$ & millilambert \\
\hline $\begin{array}{l}\text { lb/bhp- } \\
\quad \mathrm{hr}\end{array}$ & $\begin{array}{l}\text { pounds par brake horsepowar- } \\
\text { hour }\end{array}$ & $\begin{array}{l}\text { MLLW } \\
\mathrm{mm}\end{array}$ & $\begin{array}{l}\text { mean lower low water } \\
\text { millimeter }\end{array}$ \\
\hline $\mathrm{lb} / \mathrm{cu}$ & pounds per cubic foot & $\operatorname{mmf}$ & magnetomotive force \\
\hline Et & & $\mathrm{mmu}$ & mil1i-mass units \\
\hline $1 b-f t$ & pound-foot & $\mathrm{mM}$ & millimole \\
\hline $1 \mathrm{~b} / \mathrm{hr}$ & pounds per hour & $\mathrm{mp}$ & melting point \\
\hline lb-in. & pound-inch & mph & miles per hour \\
\hline $1 \mathrm{~cm}$ & least common multiple & mphps & miles per hour per second \\
\hline LF & low Erequency & $m r$ & milliroentgen \\
\hline $1 \mathrm{im}$ & limit & mrad & millirad \\
\hline $\operatorname{lin}$ & linear & mrem & nillirem (roentgen equivalent \\
\hline $\operatorname{lin} \mathrm{ft}$ & linear foot & & $\operatorname{man})$ \\
\hline $1 \mathrm{iq}$ & liquid & mrep & millirep (roentgen equivalent \\
\hline $1 \mathrm{~m}$ & lumen & & physical) \\
\hline $\operatorname{lm}-\mathrm{hr}$ & lumen-hour & $\mathrm{ms}$ & manuscript \\
\hline $\operatorname{lmtd}$ & $\begin{array}{l}\text { log mean temperature } \\
\text { difference }\end{array}$ & $\begin{array}{l}\text { Ms } \\
\text { msec }\end{array}$ & $\begin{array}{l}\text { megasecond } \\
\text { millisecond }\end{array}$ \\
\hline Ltd . & limited (as a title) & Mt & megaton \\
\hline $\log$ & logarithm, common & $\mathrm{mv}$ & millivolt \\
\hline $\log _{e}$ & logarithm, natural & $\mathrm{Mv}$ & million volts \\
\hline long. & longitude & mva & millivolt-ampere \\
\hline $10 x$ & liquid oxygen & $\mathrm{Mw}$ & megawatt \\
\hline $1-\mathrm{p}$ & low-pressure (adj) & Mwe & megawatt electric \\
\hline $1 \mathrm{pw}$ & lumens per watt & Mwt & megawatt thermal \\
\hline lwm & low water mark & $\mathrm{Mx}$ & maxwe 11 \\
\hline & & $\mathrm{Mu}$ & millimicron \\
\hline $\mathrm{m}$ & meter & & \\
\hline $\mathrm{m}$. & minute (astronomica 1 ) & $\mathrm{n}$ & newton \\
\hline M & thousand & $\mathrm{N}$ & north \\
\hline ma & milliampere & $\mathrm{nm}$ & nuclear magneton \\
\hline MAC & maximum allowable concentration & $\mathrm{n} \cdot \mathrm{mi}$. & nautical mile \\
\hline math & mathematics, -ical & no. & number \\
\hline $\max$ & maximum & $\mathrm{Np}$ & neper \\
\hline $\mathrm{mb}$ & millibar & nsec & nanosecond \\
\hline $\mathrm{mB}$ & millibarn & NSSS & nuclear steam supply system \\
\hline $\mathrm{mc}$ & millicurie & & \\
\hline Mc & megacurie & OD & outside diameter \\
\hline MC & megacycle & oe & oersted \\
\hline $\operatorname{Mcf}$ & thousand cubic feet & ohm-cm & ohm-centimeter \\
\hline $\mathrm{MCM}$ & thousand circular mils & oz & ounce \\
\hline mep & mean effective pressure & $o z-f t$ & ounce-foot \\
\hline Mev & million electron volts & oz-in. & ounce-inch \\
\hline $\mathrm{mf}$ & millifarad & & \\
\hline MF & medium frequency & $\mathrm{p}$. & page \\
\hline $\mathrm{mg}$ & miligram & $\mathrm{P}$ & poise \\
\hline $\mathrm{mG}$ & milligauss & par. & paragraph \\
\hline mgd & million gallons per day & $\mathrm{PD}$ & potential difference \\
\hline $\mathrm{mh}$ & millihenry & pe & probable error \\
\hline
\end{tabular}




\begin{tabular}{|c|c|c|c|}
\hline $\mathrm{pf}$ & power factor, plant factor & $\operatorname{sp} g r$ & specific gravity \\
\hline $\mathrm{pF}$ & picofarad, water energy & sp ht & specific heat \\
\hline $\mathrm{ph}$ & phase & $\mathrm{sq}$ & square \\
\hline $\mathrm{pH}$ & hydrogen-ion concentration & $\mathrm{sq} \mathrm{cm}$ & square centimeter \\
\hline $\mathrm{PNdb}$ & perceived noise in decibels & sq $\mathrm{ft}$ & square foot \\
\hline $\mathrm{ppb}$ & parts per billion & sq in. & square inch \\
\hline ppm & parts per million & $\mathrm{sq} \mathrm{km}$ & square kilometer \\
\hline PPI & plan position indicator & $\mathrm{sq} \mathrm{m}$ & square meter \\
\hline ps $\mathrm{f}$ & pounds per square foot & $\mathrm{sq} \mathrm{mm}$ & square millimeter \\
\hline psi & pounds per square inch & squ & square micron \\
\hline psia & pounds per square inch absolute & $\mathrm{sr}$ & steradian \\
\hline psid & $\begin{array}{l}\text { pounds per square inch } \\
\text { differential }\end{array}$ & $\begin{array}{l}\text { S.S. } \\
\text { st }\end{array}$ & $\begin{array}{l}\text { stainless steel } \\
\text { stere }\end{array}$ \\
\hline psig & pounds per square inch gage & sta & station \\
\hline pt & pint & std & standard \\
\hline PVC & polyviny 1 chloride & STP & standard temperature and \\
\hline PWR & pressurized water reactor & & pressure \\
\hline Q & billion billion Btu & $\mathrm{T}$ & tesla \\
\hline$q \mathrm{t}$ & quart & tach & tachometer \\
\hline & & $\tan$ & tangent \\
\hline $\mathbf{r}$ & roentgen & tds & total dissolved solids \\
\hline rad & radius & tech & technical \\
\hline RBE & relative biological effectiveness & tel 1 & telephone \\
\hline ref & reference & TEL & tetraethyl lead \\
\hline rem & roentgen equivalent man & temp & temperature \\
\hline rep & roentgen equivalent physical & term. & termina 1 \\
\hline rev & revolution & thp & thrust horsepower \\
\hline rev. & revision & $\mathrm{t} \operatorname{lm}$ & telemeter \\
\hline $\mathrm{RF}$ & radio-frequency (adj) & $\mathrm{t} \cdot \mathrm{m}$. & true mean \\
\hline rms & root mean square & tol & tolerance \\
\hline rpm & revolutions per minute & tph & tons per hour \\
\hline rps & revolutions per second & ts & tensile strength \\
\hline RR & railroad & TTD & terminal temperature \\
\hline Ry & rydberg & & difference \\
\hline & & typ & typica 1 \\
\hline s & second (unit of time) & & \\
\hline S & South & UHF & ultra high frequency \\
\hline sat. & saturated & UL & Underwriters Laboratory \\
\hline scfm & standard cubic foot per minute & uv & ultraviolet \\
\hline $\begin{array}{l}\operatorname{scp} \\
\sec \end{array}$ & $\begin{array}{l}\text { sperical candlepower } \\
\text { secant }\end{array}$ & $\mathrm{v}$ & voit \\
\hline $\sec t$ & section & va & volt-ampere \\
\hline$s f c$ & specific fuel consumption & vac & vacuum \\
\hline $\mathrm{sfm}$ & surface feet per minute & $\mathrm{vc}$ & volt-coulomb \\
\hline SGR & sodium graphite reactor & ve 1 & velocity \\
\hline sh & sheet & vers & versed sine \\
\hline shp & shaft horsepower & vert & vertical \\
\hline sic & thus & VHF & very high frequency \\
\hline $\sin$ & sine & VI & viscosity index \\
\hline $\sin$ & sine of the amplitude & VLF & very low frequency \\
\hline SNAP & $\begin{array}{l}\text { Systems for Nuclear Auxiliary } \\
\text { Power }\end{array}$ & $\begin{array}{l}\text { vm } \\
\text { vol }\end{array}$ & $\begin{array}{l}\text { voltmeter } \\
\text { volume }\end{array}$ \\
\hline SP & static pressure & vp & velocity pressure \\
\hline
\end{tabular}




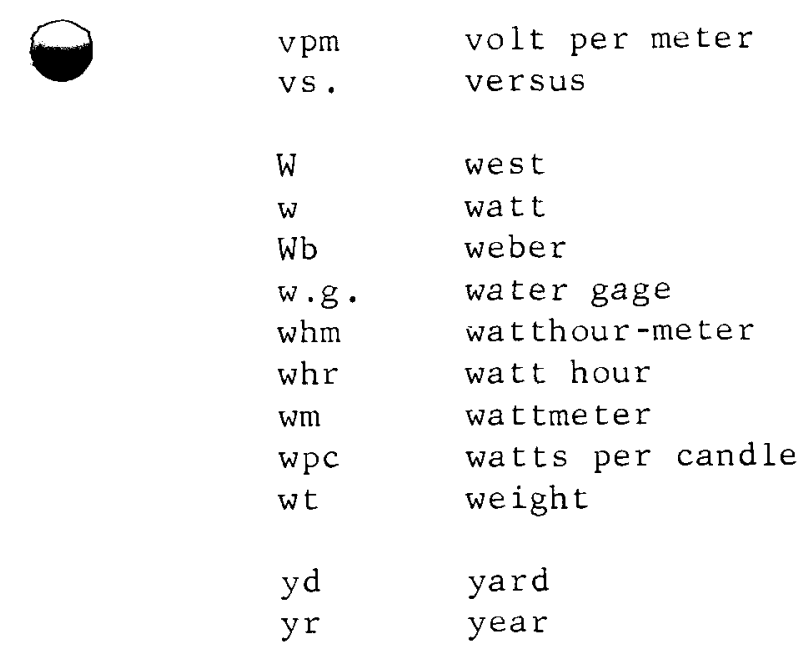

